This Supplemental Material accompanies Sharman, G.R., Stockli, D.F., Flaig, P., Raynolds, R.G., Dechesne, M., and Covault, J.A., 2021, Tectonic influence on axial-transverse sediment routing in the Denver Basin, in Craddock, J.P., Malone, D.H., Foreman, B.Z., and Konstantinou, A., eds., Tectonic Evolution of the Sevier-Laramide Hinterland, Thrust Belt, and Foreland, and Postorogenic Slab Rollback (180-20 Ma): Geological Society of America Special Paper 555, https://doi.org/10.1130/2021.2555(11).

\title{
Tectonic influence on axial-transverse sediment routing in the Denver Basin
}

Glenn R. Sharman, Daniel F. Stockli, Peter Flaig, Robert G. Raynolds, Marieke Dechesne, and Jacob A. Covault

\section{Supplemental Materials}

Appendix A: Measured sections and DB-D2-10 sample location from Bijou Creek

Table SM1: U-Pb Ages of Detrital Zircons

Table SM2: Detrital Zircon U-Pb Secondary Standard Results

Table SM3: Detrital Zircon (U-Th[Sm])/He Analytical Results

Table SM4: Non-negative Matrix Factorization Results

Table SM5: Sample Groups for End-member Comparison

Table SM6: Sample Lithology 
Appendix A. Measured sections and DB-D2-10 sample location from Bijou Creek

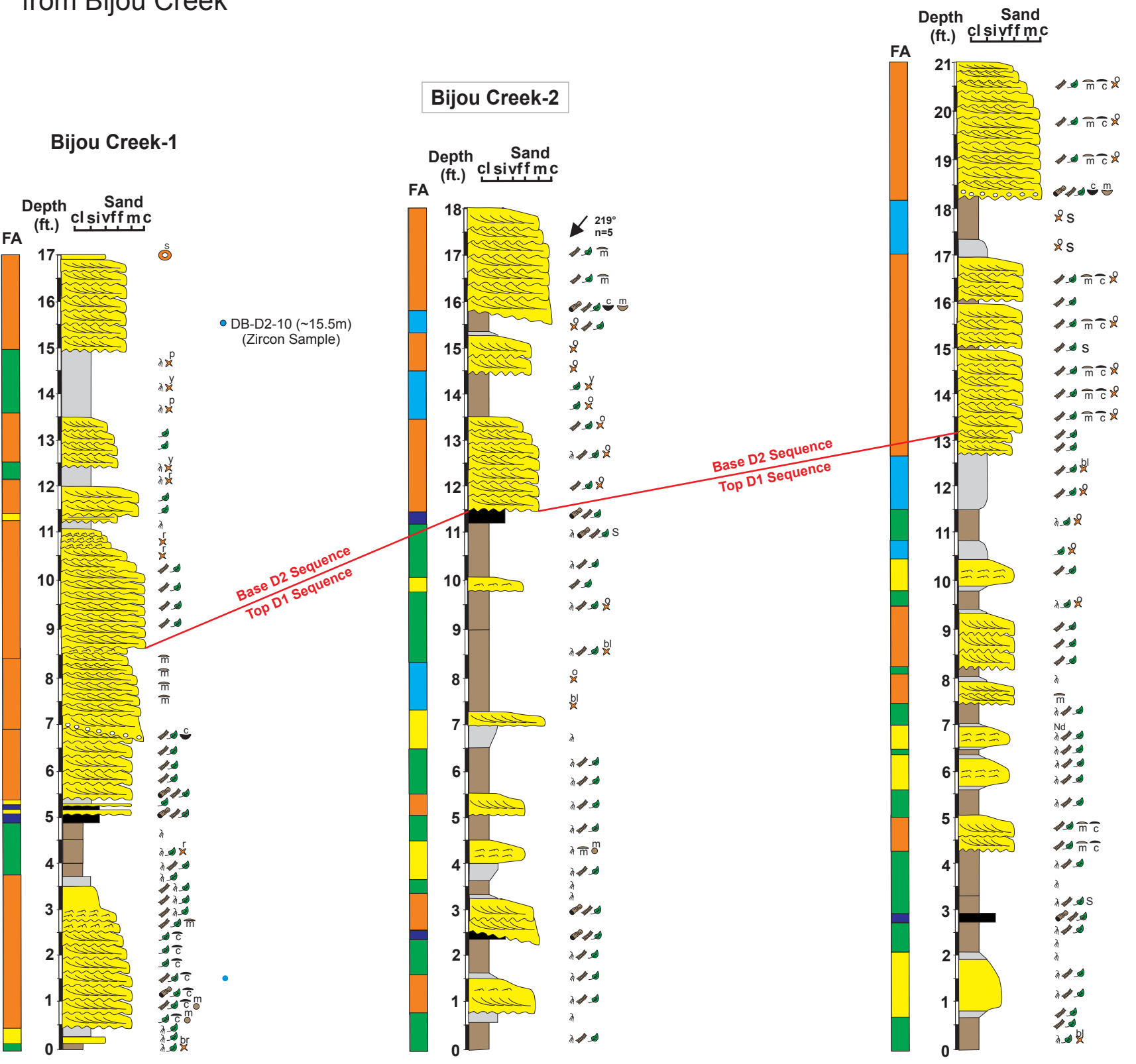

\section{Bijou Creek-3}

Symbols Key

\begin{tabular}{|c|c|}
\hline & Sandstone \\
\hline & Siltstone \\
\hline & Mudstone \\
\hline & Coal \\
\hline$\infty$ & Erosional contact \\
\hline & $\begin{array}{l}\text { Trough cross- } \\
\text { stratification }\end{array}$ \\
\hline & $\begin{array}{l}\text { Current ripple } \\
\text { cross-lamination }\end{array}$ \\
\hline & Horizontal lamination \\
\hline & Rhizoliths \\
\hline 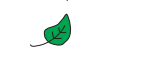 & $\begin{array}{l}\text { Leaf and/or plant } \\
\text { fragments }\end{array}$ \\
\hline$\Leftrightarrow$ & Wood impressions \\
\hline & Logs/Trees \\
\hline$m$ & Mud Rip-up Clasts \\
\hline & Organic Rip-up Clasts \\
\hline $\mathrm{m}$ & Mud Drapes \\
\hline$m$ & Mud balls \\
\hline$\frac{p}{x}$ & Mottles \\
\hline $\begin{array}{l}p=\text { purple, } \\
r=\text { red }, b r\end{array}$ & $\begin{array}{l}y=\text { yellow, o = orange } \\
=\text { brown, } b l=\text { blue }\end{array}$ \\
\hline & Siderite Cement \\
\hline S & Sulfur \\
\hline $\mathrm{Nd}$ & $\begin{array}{l}\text { Naktodemasis } \\
\text { Burrow }\end{array}$ \\
\hline & $\begin{array}{l}\text { Fluvial } \\
\text { Channel }\end{array}$ \\
\hline & $\begin{array}{l}\text { Levee or } \\
\text { Splay }\end{array}$ \\
\hline & $\begin{array}{l}\text { Lake or } \\
\text { Pond }\end{array}$ \\
\hline & Swamp \\
\hline & Paleosol \\
\hline
\end{tabular}

555-11 SM, p. 2 
Table SM1: U-Pb Ages of Detrital Zircons

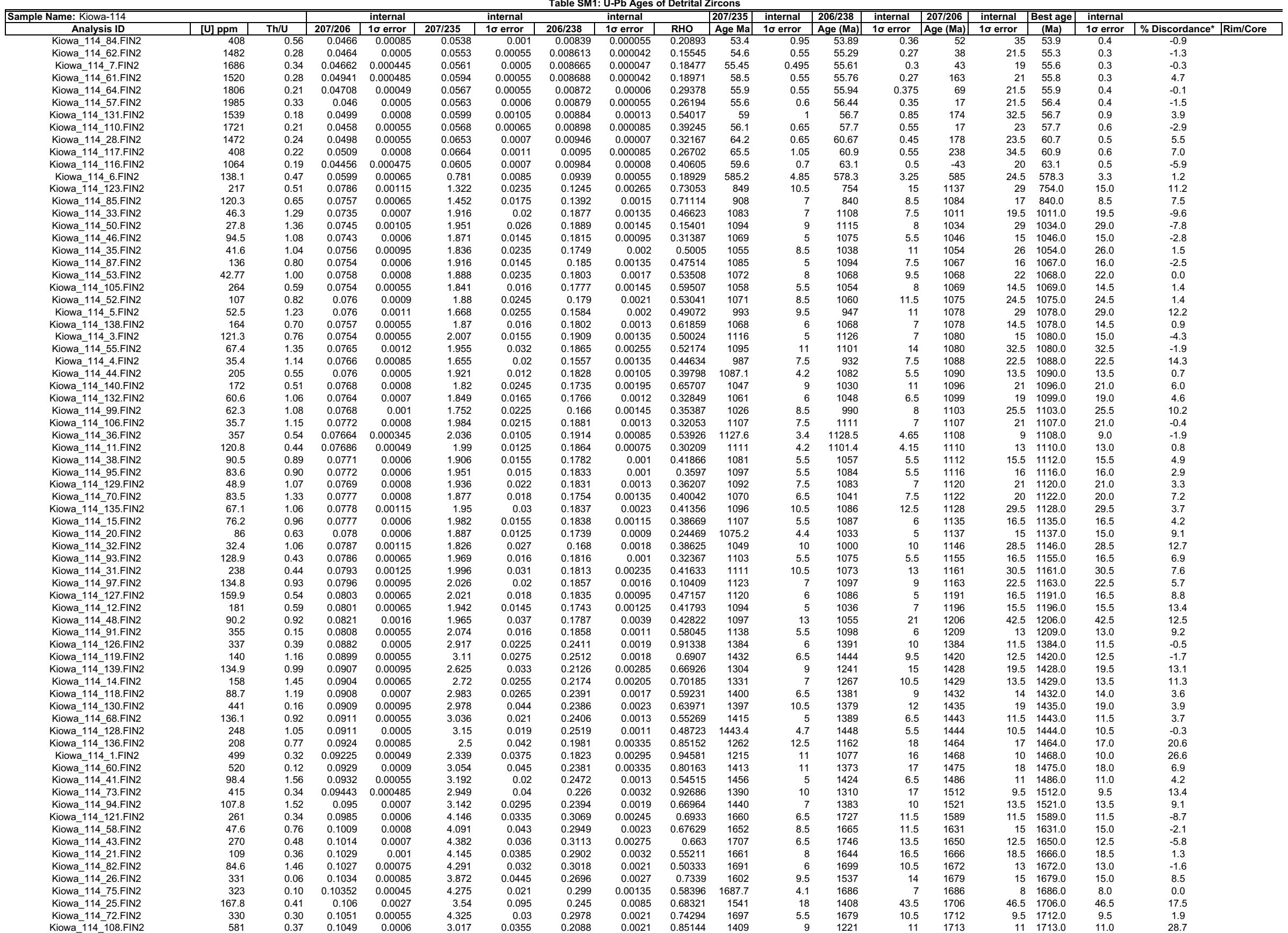




\begin{tabular}{|c|c|c|c|c|c|c|c|c|c|c|c|c|c|c|c|c|c|c|}
\hline Kiowa_114_13.FIN2 & 255 & 0.28 & 0.1058 & 0.0008 & 3.97 & 0.06 & 0.2714 & 0.0038 & 0.87966 & 1624 & 12.5 & 1544 & 19.5 & 1720 & 14 & 1720.0 & 14.0 & 10.2 \\
\hline Kiowa_114_69.FIN2 & 337 & 0.49 & 0.1061 & 0.00075 & 3.956 & 0.038 & 0.2707 & 0.00285 & 0.7644 & 1622 & 7.5 & 1542 & 14.5 & 1727 & 13 & 1727.0 & 13.0 & 10.7 \\
\hline Kiowa_114_19.FIN2 & 313 & 0.42 & 0.1062 & 0.0009 & 4.348 & 0.039 & 0.2946 & 0.00265 & 0.61484 & 1699 & 7.5 & 1663 & 13.5 & 1728 & 15 & 1728.0 & 15.0 & 3.8 \\
\hline Kiowa_114_27.FIN2 & 444 & 0.35 & 0.1064 & 0.00075 & 3.963 & 0.038 & 0.2681 & 0.0023 & 0.66181 & 1623 & 8 & 1530 & 11.5 & 1731 & 13.5 & 1731.0 & 13.5 & 11.6 \\
\hline Kiowa_114_134.FIN2 & 220 & 1.00 & 0.1064 & 0.00065 & 4.365 & 0.0275 & 0.2997 & 0.00175 & 0.50843 & 1704 & 5 & 1689 & 8.5 & 1732 & 11 & 1732.0 & 11.0 & 2.5 \\
\hline Kiowa_114_22.FIN2 & 331 & 0.17 & 0.1069 & 0.0009 & 4.32 & 0.0365 & 0.2898 & 0.00285 & 0.57573 & 1695 & 7 & 1638 & 14 & 1734 & 16 & 1734.0 & 16.0 & 5.5 \\
\hline Kiowa_114_56.FIN2 & 370 & 0.04 & 0.1068 & 0.001 & 3.6 & 0.065 & 0.2443 & 0.0044 & 0.87542 & 1536 & 14.5 & 1403 & 22.5 & 1740 & 17.5 & 1740.0 & 17.5 & 19.4 \\
\hline Kiowa_114_39.FIN2 & 141.5 & 0.47 & 0.1071 & 0.00175 & 3.9 & 0.08 & 0.264 & 0.0065 & 0.75423 & 1611 & 16 & 1509 & 31.5 & 1742 & 30 & 1742.0 & 30.0 & 13.4 \\
\hline Kiowa_114_67.FIN2 & 291 & 0.17 & 0.1071 & 0.0006 & 4.188 & 0.028 & 0.2831 & 0.0021 & 0.68216 & 1670 & 5.5 & 1606 & 10.5 & 1749 & 10 & 1749.0 & 10.0 & 8.2 \\
\hline Kiowa_114_59.FIN2 & 560 & 0.44 & 0.1079 & 0.0019 & 4.41 & 0.105 & 0.295 & 0.0065 & 0.69431 & 1707 & 20 & 1664 & 31.5 & 1752 & 32 & 1752.0 & 32.0 & 5.0 \\
\hline Kiowa_114_83.FIN2 & 160.8 & 0.35 & 0.1074 & 0.0006 & 4.73 & 0.0335 & 0.3192 & 0.00235 & 0.67903 & 1771 & 6 & 1785 & 11.5 & 1752 & 10.5 & 1752.0 & 10.5 & -1.9 \\
\hline Kiowa_114_17.FIN2 & 275 & 0.33 & 0.1078 & 0.0009 & 3.55 & 0.06 & 0.2388 & 0.0044 & 0.90197 & 1528 & 14 & 1375 & 23 & 1753 & 15 & 1753.0 & 15.0 & 21.6 \\
\hline Kiowa_114_80.FIN2 & 432 & 0.18 & 0.1078 & 0.0006 & 3.41 & 0.065 & 0.2277 & 0.0038 & 0.94525 & 1492 & 14.5 & 1318 & 20 & 1760 & 10 & 1760.0 & 10.0 & 25.1 \\
\hline Kiowa_114_45.FIN2 & 134.8 & 0.35 & 0.1079 & 0.0005 & 4.653 & 0.022 & 0.3114 & 0.00145 & 0.55728 & 1757.7 & 4 & 1747 & 7 & 1761 & 8.5 & 1761.0 & 8.5 & 0.8 \\
\hline Kiowa_114_112.FIN2 & 512 & 0.58 & 0.1081 & 0.0007 & 3.595 & 0.04 & 0.2406 & 0.00245 & 0.83419 & 1543 & 8.5 & 1391 & 13 & 1762 & 11.5 & 1762.0 & 11.5 & 21.1 \\
\hline Kiowa_114_37.FIN2 & 397 & 0.52 & 0.1083 & 0.0007 & 3.851 & 0.0475 & 0.2565 & 0.0031 & 0.85492 & 1597 & 10 & 1469 & 16 & 1767 & 11.5 & 1767.0 & 11.5 & 16.9 \\
\hline Kiowa_114_66.FIN2 & 224 & 0.83 & 0.1084 & 0.00065 & 4.511 & 0.031 & 0.3018 & 0.00195 & 0.60096 & 1731 & 5.5 & 1699 & 9.5 & 1768 & 11 & 1768.0 & 11.0 & 3.9 \\
\hline Kiowa_114_92.FIN2 & 152 & 0.39 & 0.1084 & 0.00075 & 4.64 & 0.0375 & 0.31 & 0.0023 & 0.56807 & 1754 & 6.5 & 1740 & 11 & 1768 & 13 & 1768.0 & 13.0 & 1.6 \\
\hline Kiowa_114_65.FIN2 & 243.6 & 0.30 & 0.1085 & 0.0006 & 4.916 & 0.033 & 0.3281 & 0.0023 & 0.61875 & 1804 & 6 & 1828 & 11.5 & 1769 & 10.5 & 1769.0 & 10.5 & -3.3 \\
\hline Kiowa_114_81.FIN2 & 140 & 0.34 & 0.1086 & 0.00055 & 4.892 & 0.0385 & 0.3268 & 0.0022 & 0.82009 & 1800 & 6.5 & 1822 & 11 & 1771 & 9.5 & 1771.0 & 9.5 & -2.9 \\
\hline Kiowa_114_96.FIN2 & 218 & 0.27 & 0.1087 & 0.0006 & 4.596 & 0.0255 & 0.306 & 0.00175 & 0.53388 & 1747.1 & 4.7 & 1720 & 9 & 1775 & 10 & 1775.0 & 10.0 & 3.1 \\
\hline Kiowa_114_122.FIN2 & 343 & 0.60 & 0.1089 & 0.00085 & 3.898 & 0.0375 & 0.261 & 0.00245 & 0.60337 & 1610 & 8 & 1493 & 12.5 & 1777 & 15 & 1777.0 & 15.0 & 16.0 \\
\hline Kiowa_114_9.FIN2 & 309 & 0.34 & 0.1092 & 0.00075 & 4.631 & 0.0365 & 0.3036 & 0.00255 & 0.62093 & 1752 & 6.5 & 1711 & 13 & 1779 & 12.5 & 1779.0 & 12.5 & 3.8 \\
\hline Kiowa_114_124.FIN2 & 188.6 & 0.38 & 0.1098 & 0.0005 & 4.64 & 0.029 & 0.3076 & 0.002 & 0.74321 & 1755 & 5 & 1728 & 10 & 1793 & 9 & 1793.0 & 9.0 & 3.6 \\
\hline Kiowa_114_125.FIN2 & 209 & 0.29 & 0.1098 & 0.00055 & 4.531 & 0.025 & 0.3017 & 0.00185 & 0.6401 & 1735.4 & 4.55 & 1699 & 9 & 1794 & 9 & 1794.0 & 9.0 & 5.3 \\
\hline Kiowa-114__88.FIN2 & 106.6 & 0.66 & 0.11 & 0.0006 & 4.762 & 0.039 & 0.3136 & 0.00235 & 0.75864 & 1776 & 7 & 1757 & 11.5 & 1796 & 10 & 1796.0 & 10.0 & 2.2 \\
\hline Kiowa_114_24.FIN2 & 340.9 & 0.49 & 0.111 & 0.0013 & 4.75 & 0.06 & 0.3116 & 0.0038 & 0.57489 & 1773 & 10 & 1745 & 18.5 & 1799 & 22 & 1799.0 & 22.0 & 3.0 \\
\hline Kiowa_114_89.FIN2 & 107.5 & 0.39 & 0.1105 & 0.00085 & 4.711 & 0.035 & 0.3093 & 0.0024 & 0.52991 & 1767 & 6 & 1736 & 12 & 1799 & 14 & 1799.0 & 14.0 & 3.5 \\
\hline Kiowa_114_107.FIN2 & 146.2 & 0.47 & 0.1103 & 0.0006 & 4.808 & 0.037 & 0.316 & 0.0022 & 0.70632 & 1784 & 6.5 & 1769 & 11 & 1801 & 10 & 1801.0 & 10.0 & 1.8 \\
\hline Kiowa_114_42.FIN2 & 556 & 0.13 & 0.1106 & 0.0008 & 4.86 & 0.05 & 0.3168 & 0.00315 & 0.77133 & 1792 & 8.5 & 1772 & 15.5 & 1802 & 12.5 & 1802.0 & 12.5 & 1.7 \\
\hline Kiowa_114_133.FIN2 & 286 & 0.63 & 0.1105 & 0.00065 & 3.712 & 0.044 & 0.2453 & 0.00285 & 0.86864 & 1568 & 9.5 & 1412 & 14.5 & 1802 & 11 & 1802.0 & 11.0 & 21.6 \\
\hline Kiowa_114_74.FIN2 & 252.1 & 0.45 & 0.1112 & 0.0011 & 4.45 & 0.06 & 0.2906 & 0.0044 & 0.76448 & 1712 & 11.5 & 1639 & 22 & 1804 & 18.5 & 1804.0 & 18.5 & 9.1 \\
\hline Kiowa_114_30.FIN2 & 186.3 & 0.43 & 0.1111 & 0.00075 & 4.967 & 0.0365 & 0.3216 & 0.0026 & 0.68309 & 1811 & 6.5 & 1796 & 12.5 & 1810 & 12 & 1810.0 & 12.0 & 0.8 \\
\hline Kiowa_114_109.FIN2 & 180 & 0.27 & 0.1108 & 0.0006 & 5.053 & 0.031 & 0.3311 & 0.0019 & 0.60134 & 1826 & 5 & 1843 & 9 & 1811 & 9.5 & 1811.0 & 9.5 & -1.8 \\
\hline Kiowa_114_137.FIN2 & 140.6 & 0.31 & 0.1125 & 0.00175 & 4.51 & 0.06 & 0.297 & 0.0047 & 0.49396 & 1727 & 11 & 1673 & 23.5 & 1814 & 27.5 & 1814.0 & 27.5 & 7.8 \\
\hline Kiowa_114_100.FIN2 & 192.5 & 0.42 & 0.1108 & 0.0007 & 5.065 & 0.0355 & 0.332 & 0.00275 & 0.60707 & 1832 & 6.5 & 1847 & 13.5 & 1816 & 12.5 & 1816.0 & 12.5 & -1.7 \\
\hline Kiowa_114_120.FIN2 & 155.9 & 1.01 & 0.1117 & 0.0009 & 4.721 & 0.0455 & 0.3085 & 0.00275 & 0.61951 & 1767 & 8 & 1732 & 13.5 & 1819 & 14.5 & 1819.0 & 14.5 & 4.8 \\
\hline Kiowa_114_111.FIN2 & 254 & 0.44 & 0.1118 & 0.00085 & 4.596 & 0.0405 & 0.2994 & 0.00245 & 0.60771 & 1745 & 7.5 & 1687 & 12 & 1820 & 13.5 & 1820.0 & 13.5 & 7.3 \\
\hline Kiowa_114_54.FIN2 & $\begin{array}{r}327 \\
1698\end{array}$ & $\begin{array}{l}0.60 \\
0.40\end{array}$ & $\begin{array}{l}0.1119 \\
0.1118\end{array}$ & 0.00085 & 4.556 & 0.0445 & 0.2933 & 0.00265 & 0.70026 & $\begin{array}{r}1737 \\
17687\end{array}$ & 8 & 1656 & 13 & 1825 & 14 & 1825.0 & 14.0 & 9.3 \\
\hline $\begin{array}{l}\text { Kiowa-114_90.FIN2 } \\
\text { Kiowa 1414 63.FIN2 }\end{array}$ & $\begin{aligned} 169.8 \\
212\end{aligned}$ & $\begin{array}{l}0.40 \\
0.44\end{array}$ & $\begin{array}{l}0.1118 \\
0.113\end{array}$ & $\begin{array}{l}0.00055 \\
0.00145\end{array}$ & $\begin{array}{r}4.714 \\
37\end{array}$ & $\begin{array}{r}0.023 \\
0.08\end{array}$ & $\begin{array}{l}0.3057 \\
0.237\end{array}$ & $\begin{array}{r}0.00155 \\
0.005\end{array}$ & $\begin{array}{l}0.56008 \\
0.82919\end{array}$ & $\begin{array}{r}1768.7 \\
1564\end{array}$ & $\begin{array}{l}4.05 \\
175\end{array}$ & $\begin{array}{l}1719 \\
171\end{array}$ & $\begin{array}{r}7.5 \\
26\end{array}$ & 1825 & $\begin{array}{r}8.5 \\
23.5\end{array}$ & 1825.0 & $\begin{array}{r}8.5 \\
23.5\end{array}$ & 5.8 \\
\hline $\begin{array}{l}\text { Klowa_-14_-6.1.IN2 } \\
\text { Kiowa_114_77.FIN2 }\end{array}$ & $\begin{array}{l}212 \\
192\end{array}$ & $\begin{array}{l}0.44 \\
0.39\end{array}$ & $\begin{array}{l}0.113 \\
0.113\end{array}$ & $\begin{array}{l}0.00145 \\
0.00105\end{array}$ & $\begin{array}{r}3.7 \\
4.229\end{array}$ & $\begin{array}{r}0.08 \\
0.0455\end{array}$ & $\begin{array}{l}0.231 \\
0.2715\end{array}$ & $\begin{array}{r}0.005 \\
0.0032\end{array}$ & $\begin{array}{l}0.82919 \\
0.71015\end{array}$ & $\begin{array}{l}1564 \\
1678\end{array}$ & $\begin{array}{r}17.5 \\
85\end{array}$ & $\begin{array}{l}1371 \\
1548\end{array}$ & 26 & $\begin{array}{l}1839 \\
1845\end{array}$ & $\begin{array}{r}23.5 \\
17\end{array}$ & $\begin{array}{l}1839.0 \\
-185.0\end{array}$ & $\begin{array}{l}23.5 \\
170\end{array}$ & 25.4 \\
\hline Kiowa_114_86.FIN2 & 235 & 0.69 & 0.1135 & 0.0009 & 4.23 & 0.075 & 0.265 & 0.0041 & $\begin{array}{l}0.11015 \\
0.83239\end{array}$ & $\begin{array}{l}16 / 8 \\
1668\end{array}$ & $\begin{array}{l}0.5 \\
14.5\end{array}$ & $\begin{array}{l}1548 \\
1516\end{array}$ & $\begin{array}{r}16.5 \\
21\end{array}$ & $\begin{array}{l}1845 \\
1853\end{array}$ & $\begin{array}{r}17 \\
14.5\end{array}$ & $\begin{array}{l}1845.0 \\
15530\end{array}$ & $\begin{array}{l}17.0 \\
14.5\end{array}$ & $\begin{array}{l}16.1 \\
18 ?-1\end{array}$ \\
\hline Kiowa_114_102.FIN2 & 193.4 & 0.38 & 0.1161 & 0.00085 & 5.004 & 0.0455 & 0.3128 & 0.002 & 0.6041 & 1817 & 7.5 & 1754 & 10 & 1891 & $\begin{aligned} 14.5 \\
13\end{aligned}$ & $\begin{array}{l}1853.0 \\
18910\end{array}$ & $\begin{array}{l}14.5 \\
130\end{array}$ & $\begin{array}{l}18.2 \\
72\end{array}$ \\
\hline Kiowa_114_115.FIN2 & 119.8 & 0.43 & 0.1161 & 0.001 & 4.99 & 0.05 & 0.3128 & 0.0028 & 0.61445 & 1815 & 8.5 & 1753 & 14 & 1894 & 15 & 1894.0 & 150 & 7.2 \\
\hline Kiowa_114_16.FIN2 & 165 & 0.35 & 0.1171 & 0.00125 & 4.35 & 0.06 & 0.2683 & 0.0036 & 0.68441 & 1697 & 11.5 & 1530 & 18 & 1901 & 19.5 & 1901.0 & $\begin{array}{l}15.0 \\
19.5\end{array}$ & $\begin{array}{l}1.4 \\
195\end{array}$ \\
\hline Kiowa_114_71.FIN2 & 120.3 & 0.38 & 0.1172 & 0.001 & 5.136 & 0.048 & 0.316 & 0.002 & 0.48876 & 1838 & 8 & 1772 & 10 & 1903 & 15 & 1903.0 & $\begin{array}{l}15.0 \\
15.0\end{array}$ & $\begin{array}{l}19.0 \\
69\end{array}$ \\
\hline Kiowa_114_34.FIN2 & 107.1 & 0.40 & 0.1192 & 0.00105 & 5.07 & 0.055 & 0.3069 & 0.0028 & 0.60695 & 1827 & 9 & 1724 & 14 & 1935 & 15.5 & 1935.0 & 15.5 & $\begin{array}{l}0.9 \\
109\end{array}$ \\
\hline Kiowa_114_47.FIN2 & 211.8 & 0.10 & 0.121 & 0.0023 & 4.71 & 0.095 & 0.287 & 0.006 & 0.61416 & 1759 & 18 & 1621 & 29.5 & 1941 & 32.5 & 1941.0 & 32.5 & 16.5 \\
\hline Kiowa_114_78.FIN2 & 219.7 & 0.19 & 0.179 & 0.00105 & 10.4 & 0.135 & 0.42 & 0.0055 & 0.87766 & 2462 & 12.5 & 2252 & 26 & 2639 & 9.5 & 2639.0 & 9.5 & 14.7 \\
\hline Kiowa_114_114.FIN2 & 431 & 0.30 & 0.19 & 0.00075 & 12.84 & 0.065 & 0.4907 & 0.00205 & 0.65668 & 2667.4 & 4.9 & 2575 & 9 & 2740 & 6.5 & 2740.0 & 6.5 & 6.0 \\
\hline Kiowa_-114__.FIN2 & 1687 & 0.34 & 0.0691 & 0.0013 & 0.0841 & 0.0019 & 0.00861 & 0.000065 & 0.34743 & 81.8 & 1.75 & 55.23 & 0.405 & 850 & 39.5 & DISCARD & \#VALUE! & 32.5 \\
\hline Kiowa_114_8FIN2 & 456 & 0.09 & 0.1186 & 0.00085 & 3.3 & 0.044 & 0.2019 & 0.0035 & 0.89065 & 1479 & 10.5 & 1182 & 18.5 & 1928 & 13 & DISCARD & \#VALUE! & 38.7 \\
\hline Kiowa_114_10.FIN2 & 380.1 & 0.65 & 0.1185 & 0.0011 & 2.118 & 0.0255 & 0.1291 & 0.0019 & 0.77486 & 1152 & 8.5 & 782 & 11 & 1928 & 16.5 & DISCARD & \#VALUE! & 32.1 \\
\hline Kiowa_114_18.FIN2 & 414 & 0.26 & 0.1378 & 0.00255 & 2.33 & 0.055 & 0.1259 & 0.0032 & 0.67894 & 1212 & 16.5 & 761 & 18 & 2150 & 33 & DISGARD & \#VALUE! & 37.2 \\
\hline Kiowa_114_29.FIN2 & 178.4 & 0.16 & 0.126 & 0.00175 & 2.81 & 0.055 & 0.162 & 0.00305 & 0.56046 & 1351 & 74.5 & 966 & 17 & 2027 & 24.5 & DISGARD & \#VALUE! & 52.3 \\
\hline Kiowa_114_40.FIN2 & 295 & 0.55 & 0.0953 & 0.0029 & 1.21 & 0.0385 & 0.0949 & 0.0012 & 0.43404 & 787 & 16 & 584 & 7 & 1430 & 55 & DISGARD & \#VALUE! & 25.8 \\
\hline Kiowa_114_49.FIN2 & 493 & 0.65 & 0.1043 & 0.00115 & 1.266 & 0.025 & 0.0875 & 0.0019 & 0.85567 & 822 & 10.5 & 539 & 11 & 1683 & 20.5 & DISCARD & \#VALUE! & 34.4 \\
\hline Kiowa_114_51.FIN2 & 590 & 0.35 & 0.1264 & 0.00085 & 3.745 & 0.039 & 0.215 & 0.0027 & 0.8269 & 15 & 8.5 & 1253 & 14 & 2045 & $12.5 \mathrm{P}>\mathrm{C}$ & DISCARD & \#VALUE! & 38.7 \\
\hline Kiowa_114_98.FIN2 & 381 & 0.48 & 0.1035 & 0.0008 & 2.768 & 0.024 & 0.195 & 0.002 & 0.66048 & 134 & 6.5 & 1150 & 11 & 1678 & 15 & DISCARD & \#VALUE! & 31.5 \\
\hline Kiowa_114_104_FIN2 & 103.4 & 0.63 & 0.0706 & 0.0005 & 1.771 & 0.015 & 0.1822 & 0.0012 & 0.53878 & 1033 & 5.5 & 1078 & 6.5 & 936 & 15.5 & DISCARD & \#VALUE! & -15.2 \\
\hline Name: Kiowa-262 & & & & internal & & internal & & internal & & $207 / 235$ & internal & $206 / 238$ & internal & $07 / 206$ & internal & Best age & internal & \\
\hline Analysis ID & [U] ppm & $\mathrm{Th} / \mathrm{U}$ & \begin{tabular}{|l|}
$207 / 206$ \\
\end{tabular} & 10 error & 207/235 & $1 \sigma$ error & 206/238 & $1 \sigma$ error & RHO & Age Ma & $1 \sigma$ error & Age (Ma) & $1 \sigma$ error & Age (Ma) & $1 \sigma$ error & (Ma) & $1 \sigma$ error & \% Discordance $\mid$ Rim/Core \\
\hline Kiowa 262 43.FIN2 & 112.5 & 0.69 & 0.0499 & 0.0017 & 0.0747 & 0.00245 & $\frac{1}{0.01082}$ & 0.0001 & 0.19665 & 72.8 & $\frac{12.3}{2.3}$ & 69.4 & 0.65 & 150 & 60 & $\frac{6.4}{69.4}$ & 0.7 & 4.7 \\
\hline Kiowa_262_64.FIN2 & 79.5 & 0.48 & 0.0493 & 0.0018 & 0.0742 & 0.00265 & 0.01092 & 0.000125 & 0.052483 & 72.9 & 2.55 & 70 & 0.8 & 130 & 65 & 70.0 & 0.8 & 4.0 \\
\hline Kiowa_262 5.FIN2 & 61.8 & 0.51 & 0.0472 & 0.0018 & 0.0724 & 0.00275 & 0.01115 & 0.00013 & 0.14542 & 70.5 & 2.6 & 71.4 & 0.85 & 60 & 70 & 71.4 & 0.9 & -1.3 \\
\hline Kiowa_262_50.FIN2 & 292 & 0.76 & 0.0489 & 0.00095 & 0.088 & 0.00165 & 0.01302 & 0.00011 & 0.25292 & 85.5 & 1.55 & 83.4 & 0.7 & 133 & 37 & 83.4 & 0.7 & 2.5 \\
\hline Kiowa_262_20.FIN2 & 303 & 0.55 & 0.0488 & 0.00075 & 0.1091 & 0.00165 & 0.01627 & 0.00012 & 0.262 & 105 & 1.5 & 104 & 0.75 & 140 & 31 & 104.0 & 0.8 & 1.0 \\
\hline Kiowa_262_57.FIN2 & 115.4 & 0.72 & 0.0498 & 0.0009 & 0.1742 & 0.0028 & 0.02524 & 0.000165 & -0.068228 & 162.6 & 2.45 & 160.7 & 1.05 & 181 & 36 & 160.7 & 1.1 & 1.2 \\
\hline Kiowa_262_54.FIN2 & 105 & 1.26 & 0.0513 & 0.00265 & 0.181 & 0.0085 & 0.0256 & 0.0006 & 0.006846 & 168 & 7.5 & 162.7 & 3.65 & 220 & 100 & 162.7 & 3.7 & 3.2 \\
\hline Kiowa_262_67.FIN2 & 272.7 & 0.30 & 0.0509 & 0.0007 & 0.1825 & 0.0025 & 0.02579 & 0.00015 & 0.18405 & 169.9 & 2.15 & 164.2 & 0.95 & 221 & 28.5 & 164.2 & 1.0 & 3.4 \\
\hline Kiowa_262_41.FIN2 & 574 & 0.59 & 0.04935 & 0.00047 & 0.1769 & 0.0015 & 0.02581 & 0.00012 & 0.086716 & 165.3 & 1.3 & 164.3 & 0.75 & 158 & 20 & 164.3 & 0.8 & 0.6 \\
\hline Kiowa_262_7.FIN2 & 444 & 0.98 & 0.0506 & 0.00055 & 0.1829 & 0.002 & 0.02631 & 0.00013 & 0.31135 & 170.3 & 1.7 & 167.4 & 0.8 & 209 & 22 & 167.4 & 0.8 & 1.7 \\
\hline Kiowa_262_40.FIN2 & 697 & 1.06 & 0.05151 & 0.000395 & 0.2093 & 0.00155 & 0.02911 & 0.00014 & 0.30784 & 192.8 & 1.3 & 185 & 0.9 & 254 & 17 & 185.0 & 0.9 & 4.0 \\
\hline Kiowa_262_34.FIN2 & 288 & 0.50 & 0.055 & 0.00055 & 0.4087 & 0.00415 & 0.05321 & 0.000345 & 0.38208 & 347.3 & & 334.1 & 2.1 & 399 & 22 & 334.1 & 2.1 & 3.8 \\
\hline
\end{tabular}




\begin{tabular}{|c|c|c|c|c|c|c|c|c|c|c|c|c|c|c|c|c|c|c|c|}
\hline Kiowa_262_31.FIN2 & 50.2 & 0.65 & 0.0563 & 0.00115 & 0.437 & 0.0085 & 0.0561 & 0.0005 & 0.24905 & 366 & 6 & 352 & 3.05 & 410 & 40.5 & 352.0 & 3.1 & 3.8 & \\
\hline Kiowa_262_61.FIN2 & 438 & 0.33 & 0.0561 & 0.000325 & 0.5286 & 0.00315 & 0.06776 & 0.000315 & 0.45738 & 431.1 & 2.15 & 422.6 & 1.9 & 449 & 13 & 422.6 & 1.9 & 2.0 & \\
\hline Kiowa_262_13.FIN2r & 349 & 0.04 & 0.0584 & 0.00065 & 0.595 & 0.007 & 0.0742 & 0.00095 & 0.58794 & 473.4 & 4.45 & 462 & 5.5 & 550 & 27.5 & 462.0 & 5.5 & 2.4 & Rim \\
\hline Kiowa_262_46.FIN2 & 268 & 0.01 & 0.0558 & 0.0005 & 0.59 & 0.0055 & 0.0758 & 0.000495 & 0.29266 & 469.9 & 3.4 & 470.9 & 2.95 & 432 & 21 & 470.9 & 3.0 & -0.2 & \\
\hline Kiowa_262_32.FIN2 & 69.5 & 0.91 & 0.0608 & 0.00075 & 0.738 & 0.008 & 0.08703 & 0.00046 & 0.089078 & 559.7 & 4.8 & 537.9 & 2.75 & 616 & 25.5 & 537.9 & 2.8 & 3.9 & \\
\hline Kiowa_262_35.FIN2 & 401 & 0.53 & 0.06186 & 0.000335 & 0.898 & 0.006 & 0.1045 & 0.00055 & 0.58392 & 649.8 & 3.25 & 640.3 & 3.15 & 663 & 11.5 & 640.3 & 3.2 & 1.5 & \\
\hline Kiowa_262_2.FIN2 & 13.99 & 0.53 & 0.0792 & 0.0016 & 1.497 & 0.0335 & 0.1377 & 0.002 & 0.45288 & 920 & 13.5 & 830 & 11.5 & 1140 & 41.5 & 830.0 & 11.5 & 9.8 & \\
\hline Kiowa_262_24.FIN2 & 541.1 & 0.32 & 0.06937 & 0.000495 & 1.42 & 0.0115 & 0.1479 & 0.00115 & 0.65372 & 896.3 & 4.95 & 889 & 6.5 & 902 & 14.5 & 902.0 & 14.5 & 1.4 & \\
\hline Kiowa_262_44.FIN2 & 142.4 & 0.32 & 0.0711 & 0.0006 & 1.535 & 0.014 & 0.1556 & 0.0011 & 0.47911 & 942 & 5.5 & 934 & 6 & 945 & 18 & 945.0 & 18.0 & 1.2 & \\
\hline Kiowa_262_14.FIN2 & 36.4 & 0.48 & 0.0726 & 0.0009 & 1.647 & 0.0185 & 0.1652 & 0.00115 & 0.15908 & 985 & 7 & 985 & 6.5 & 981 & 25 & 981.0 & 25.0 & -0.4 & \\
\hline $\begin{array}{l}\text { Kiowa_262_63.FIN2 } \\
\text { Kiowa 262 } 9 \text { FIN2 }\end{array}$ & 39.7 & 0.00 & 0.0756 & 0.00075 & 1.876 & 0.02 & 0.1778 & 0.00105 & 0.2984 & 1069 & 7 & 1055 & 6 & 1065 & 20.5 & 1065.0 & 20.5 & 0.9 & \\
\hline $\begin{array}{l}\text { Kiowa_262_9.FIN2 } \\
\text { Kiowa 262 39FIN2 }\end{array}$ & 107.8 & $\begin{array}{l}0.70 \\
0.54\end{array}$ & $\begin{array}{l}0.0756 \\
0.0759\end{array}$ & 0.00055 & 1.868 & 0.014 & 0.1803 & 0.00085 & $\begin{array}{r}0.3672 \\
0.5103\end{array}$ & 1069.5 & 4.75 & 1068.2 & 4.7 & 1079 & 14 & 1079.0 & 14.0 & 1.0 & \\
\hline & 221 & 0.54 & 0.0759 & 0.00055 & 2.045 & 0.016 & 0.1939 & 0.0015 & 0.56103 & 1129 & 5.5 & 1142 & 8 & 1084 & 14.5 & 1084.0 & 14.5 & -5.4 & \\
\hline $\begin{array}{l}\text { Kiowa-262-36. } 6 \text {. IN2 } \\
\text { Kiowa 262 8IFIN2 }\end{array}$ & $\begin{array}{l}276 \\
57.8\end{array}$ & 0.52 & $\begin{array}{l}0.0757 \\
0.0772\end{array}$ & $\begin{array}{l}0.0006 \\
0.0007\end{array}$ & $\begin{array}{r}1.505 \\
1.875\end{array}$ & $\begin{array}{r}0.012 \\
0.0215\end{array}$ & $\begin{array}{l}0.1427 \\
0.1771\end{array}$ & $\begin{array}{r}0.00115 \\
0.0015\end{array}$ & $\begin{array}{l}0.47303 \\
0.58758\end{array}$ & 931.2 & 4.9 & $\begin{array}{r}859 \\
1050-10\end{array}$ & $\begin{array}{r}6.5 \\
8.5\end{array}$ & 1087 & $\begin{array}{r}16.5 \\
19\end{array}$ & 1087.0 & 16.5 & 21.0 & \\
\hline $\begin{array}{l}\text { Kowa_26_-8.FIN2 } \\
\text { Kiowa_262_33.FIN2 }\end{array}$ & $\begin{array}{l}51.0 \\
84.8\end{array}$ & 1.06 & 0.0773 & $\begin{array}{l}0.0007 \\
0.00055\end{array}$ & $\begin{array}{l}1.885 \\
1.977\end{array}$ & $\begin{array}{l}0.0215 \\
0.0145\end{array}$ & 0.17711 & $\begin{array}{r}0.0015 \\
0.00085\end{array}$ & 0.58758 & $\begin{array}{l}1070 \\
1106\end{array}$ & $\begin{array}{r}7.5 \\
5\end{array}$ & $\begin{array}{r}1050 \\
10868\end{array}$ & 8.5 & 1114 & 19 & $\begin{array}{l}114.0 \\
11470\end{array}$ & 19.0 & 5.7 & \\
\hline Kiowa_262_19.FIN2 & 140.2 & 0.20 & 0.0784 & 0.00105 & 2.048 & 0.0295 & 0.1897 & $\begin{array}{l}0.00085 \\
0.00255\end{array}$ & $\begin{array}{l}0.536353 \\
0.53673\end{array}$ & $\begin{array}{l}11106 \\
1130\end{array}$ & $\begin{array}{r}5 \\
9.5\end{array}$ & $\begin{array}{r}1086.8 \\
1119\end{array}$ & $\begin{array}{r}4.65 \\
14\end{array}$ & $\begin{array}{l}1112 \\
1150\end{array}$ & $\begin{array}{r}14 \\
265\end{array}$ & $\begin{array}{l}1127.0 \\
11500\end{array}$ & $\begin{array}{r}14.0 \\
26.5\end{array}$ & $\begin{array}{l}3.6 \\
27\end{array}$ & \\
\hline Kiowa_262_15.FIN2 & 216 & 0.22 & 0.07894 & 0.000475 & 1.968 & 0.013 & 0.1809 & 0.0008 & 0.4633 & 1103.4 & 4.5 & 1071.4 & 4.5 & 1167 & 12.5 & 1167.0 & 12.5 & 8.2 & \\
\hline Kiowa_262_12.FIN2 & 82.3 & 1.26 & 0.0794 & 0.0008 & 1.709 & 0.0195 & 0.157 & 0.0016 & 0.55613 & 1009 & 7 & 939 & 9 & 1174 & 19.5 & 1174.0 & 19.5 & 20.0 & \\
\hline Kiowa_262_51.FIN2 & 148.1 & 0.37 & 0.0827 & 0.00055 & 2.38 & 0.0175 & 0.2069 & 0.00155 & 0.60595 & 1237 & 5.5 & 1212 & 8.5 & 1257 & 13.5 & 1257.0 & 13.5 & 3.6 & \\
\hline Kiowa_262_53.FIN2 & 100.5 & 1.17 & 0.0846 & 0.00075 & 2.102 & 0.0195 & 0.1789 & 0.00115 & 0.3615 & 1148 & 6.5 & 1060 & 6.5 & 1296 & 18.5 & 1296.0 & 18.5 & 18.2 & \\
\hline Kiowa_262_11.FIN2r & 347 & 0.37 & 0.0849 & 0.00065 & 2.361 & 0.0475 & 0.2022 & 0.0037 & 0.80046 & 1230 & 14 & 1187 & 20 & 1312 & 15 & 1312.0 & 15.0 & 9.5 & Rim \\
\hline Kiowa_262_13.FIN2C & 118.7 & 0.26 & 0.0858 & 0.00105 & 1.825 & 0.0315 & 0.1548 & 0.0019 & 0.68274 & 1052 & 11 & 927 & 10.5 & 1324 & 23.5 & 1324.0 & 23.5 & 30.0 & Core \\
\hline Kiowa_262_17.FIN2 & 626 & 0.19 & 0.0862 & 0.00075 & 2.113 & 0.0205 & 0.1785 & 0.00175 & 0.6413 & 1150 & 6.5 & 1058 & 9.5 & 1329 & 16.5 & 1329.0 & 16.5 & 20.4 & \\
\hline Kiowa_262_25.FIN2 & 138.1 & 0.82 & 0.08633 & 0.000445 & 2.757 & 0.0145 & 0.2305 & 0.0009 & 0.3334 & 1343.9 & 3.95 & 1336.7 & 4.8 & 1344 & 10.5 & 1344.0 & 10.5 & 0.5 & \\
\hline Kiowa_262_52.FIN2 & 222 & 0.98 & 0.08877 & 0.000385 & 2.994 & 0.0155 & 0.2419 & 0.00115 & 0.65878 & 1405 & 3.85 & 1396 & 6 & 1396 & 8.5 & 1396.0 & 8.5 & 0.0 & \\
\hline Kiowa_-262_4.FIN2 & 271.4 & 1.01 & 0.09491 & 0.000405 & 3.365 & 0.016 & 0.2582 & 0.001 & 0.56983 & 1495.3 & 3.75 & 1480 & 5 & 1523 & 8 & 1523.0 & 8.0 & 2.8 & \\
\hline Kiowa_262_48.FIN2 & 632 & 0.49 & 0.10048 & 0.000455 & 4.058 & 0.016 & 0.2904 & 0.00155 & 0.59829 & 1645.3 & 3.2 & 1643 & 7.5 & 1630 & 8.5 & 1630.0 & 8.5 & -0.8 & \\
\hline Kiowa_262_65.FIN2 & 262 & 0.60 & 0.10222 & 0.00042 & 4.41 & 0.0265 & 0.3096 & 0.00155 & 0.73663 & 1712 & 5 & 1738 & 7.5 & 1662 & 7.5 & 1662.0 & 7.5 & -4.6 & \\
\hline Kiowa_262_45.FIN2 & 148 & 0.54 & 0.1026 & 0.00085 & 4.278 & 0.042 & 0.2995 & 0.00285 & 0.58351 & 1687 & 8 & 1690 & 14.5 & 1670 & 15.5 & 1670.0 & 15.5 & -1.2 & \\
\hline Kiowa_262_30.FIN2 & 104.4 & 1.06 & 0.103 & 0.0006 & 4.027 & 0.0305 & 0.2814 & 0.00225 & 0.71318 & 1637 & 6 & 1603 & 10.5 & 1674 & 10.5 & 1674.0 & 10.5 & 4.2 & \\
\hline Kiowa_262_21.FIN2 & 378 & 0.15 & 0.1035 & 0.00055 & 4.265 & 0.029 & 0.2985 & 0.0022 & 0.73201 & 1685 & 5.5 & 1683 & 11 & 1684 & 9.5 & 1684.0 & 9.5 & 0.1 & \\
\hline Kiowa_262_55.FIN2 & 673 & 0.27 & 0.1039 & 0.0007 & 4.209 & 0.025 & 0.2908 & 0.00215 & 0.50959 & 1679 & 5 & 1645 & 11 & 1690 & 13 & 1690.0 & 13.0 & 2.7 & \\
\hline Kiowa_262_11.FIN2c & 682 & 0.02 & 0.10401 & 0.00032 & 3.778 & 0.018 & 0.2635 & 0.0012 & 0.78573 & 1588.4 & 3.75 & 1508 & 6 & 1698 & 5.5 & 1698.0 & 5.5 & 11.2 & Core \\
\hline Kiowa_262_18.FIN2 & 278 & 0.48 & 0.10546 & 0.00045 & 4.632 & 0.0275 & 0.3183 & 0.00175 & 0.71811 & 1753 & 5 & 1781 & 8.5 & 1720 & 8 & 1720.0 & 8.0 & -3.5 & \\
\hline Kiowa_262_22.FIN2 & 244 & 0.33 & 0.10574 & 0.000425 & 4.315 & 0.0185 & 0.2953 & 0.0013 & 0.58593 & 1696.2 & 3.6 & 1668 & 6.5 & 1725 & 7.5 & 1725.0 & 7.5 & 3.3 & \\
\hline Kiowa_262_27.FIN2 & $\begin{array}{r}772 \\
793\end{array}$ & 0.02 & 0.1061 & 0.0006 & 4.13 & 0.05 & 0.281 & 0.0036 & 0.90348 & 1655 & 10 & 1594 & 18 & 1731 & 10 & 1731.0 & 10.0 & 7.9 & \\
\hline Kiowa_262_6.FIN2 & 79.3 & 0.33 & 0.1068 & 0.0007 & 4.68 & 0.0325 & 0.3192 & 0.0017 & 0.49375 & 1763 & 6 & 1785 & 8.5 & 1739 & 12 & 1739.0 & 12.0 & -2.6 & \\
\hline $\begin{array}{l}\text { Kiowa_262_66.FIN2 } \\
\text { Kiowa 262 1.FIN2 }\end{array}$ & 310 & $\begin{array}{l}0.31 \\
0.66\end{array}$ & 0.1073 & 0.0013 & $\begin{array}{r}3.77 \\
374\end{array}$ & 0.07 & $\begin{array}{l}0.254 \\
0.997\end{array}$ & $\begin{array}{r}0.0048 \\
\end{array}$ & 0.79298 & 1572 & 15.5 & 1453 & 25 & 1740 & 23 & 1740.0 & 23.0 & 16.5 & \\
\hline $\begin{array}{l}\text { Kiowa_262 } 1 \text {.1.FIN2 } \\
\text { Kiowa 262 } 3 \text {.FIN2 }\end{array}$ & $\begin{array}{r}86 \\
315\end{array}$ & $\begin{array}{l}0.66 \\
0.33\end{array}$ & $\begin{array}{l}0.1084 \\
0.1086\end{array}$ & $\begin{array}{l}0.0007 \\
0.0006\end{array}$ & $\begin{array}{r}4.374 \\
4.25\end{array}$ & $\begin{array}{l}0.033 \\
0.055\end{array}$ & 0.2947 & $\begin{array}{l}0.00165 \\
0.00375\end{array}$ & $\begin{array}{l}0.54429 \\
0.90424\end{array}$ & $\begin{array}{l}1705 \\
165\end{array}$ & $\begin{array}{l}6.5 \\
11\end{array}$ & $\begin{array}{l}1664 \\
1591\end{array}$ & $\begin{array}{l}8.5 \\
19\end{array}$ & 1766 & 11.5 & $\begin{array}{l}1766.0 \\
1770\end{array}$ & 11.5 & 5.8 & \\
\hline $\begin{array}{l}\text { Kiowa_262-37.FIN2 } \\
\text { Kiowa 262 58.FIN2 }\end{array}$ & $\begin{array}{r}335 \\
267.8\end{array}$ & $\begin{array}{l}0.33 \\
0.32\end{array}$ & $\begin{array}{l}0.1086 \\
0.1087\end{array}$ & $\begin{array}{r}0.0006 \\
0.00065\end{array}$ & $\begin{array}{l}4.25 \\
4.73\end{array}$ & 0.055 & 0.2807 & $\begin{array}{l}0.00375 \\
0.00175\end{array}$ & 0.90424 & $\begin{array}{l}1675 \\
1770\end{array}$ & 11 & $\begin{array}{l}1591 \\
1748\end{array}$ & 19 & 1774 & $\begin{array}{r}10 \\
115\end{array}$ & $\begin{array}{l}1774.0 \\
1760\end{array}$ & $\begin{array}{l}10.0 \\
115\end{array}$ & 10.3 & \\
\hline Kiowa_262_16.FIN2 & 494 & 0.38 & 0.122 & 0.0005 & 5.719 & $\begin{array}{l}0.0395 \\
0.0395\end{array}$ & 0.3394 & $\begin{array}{r}0.001 / 5 \\
0.0021\end{array}$ & 0.79365 & 1932 & $\begin{array}{r}6.5 \\
6\end{array}$ & $\begin{array}{l}1 / 48 \\
1883\end{array}$ & $\begin{array}{r}9 \\
10\end{array}$ & $\begin{array}{l}1 / 76 \\
1984\end{array}$ & $\begin{array}{r}11.5 \\
7.5\end{array}$ & $\begin{array}{l}1776.0 \\
1984.0\end{array}$ & $\begin{array}{l}71.5 \\
7.5\end{array}$ & $\begin{array}{l}1.6 \\
5.1\end{array}$ & \\
\hline Kiowa_262_28.FIN2 & 229.5 & 0.41 & 0.1266 & 0.00095 & 4.532 & 0.048 & 0.2578 & 0.00225 & 0.69671 & 1733 & 8.5 & 1478 & 11.5 & 2045 & 12.5 & 2045.0 & 12.5 & 27.7 & \\
\hline Kiowa_262_49.FIN2 & 23.5 & 1.65 & 0.1821 & 0.00125 & 12.02 & 0.14 & 0.475 & 0.0055 & 0.84029 & 2600 & 11 & 2497 & 25 & 2666 & 11.5 & 2666.0 & 11.5 & 6.3 & \\
\hline Kiowa_262_26.FIN2 & 112.1 & 0.07 & 0.1819 & 0.001 & 12.29 & 0.07 & 0.4861 & 0.0024 & 0.53215 & 2625 & 5.5 & 2553 & 10.5 & 2669 & 8.5 & 2669.0 & 8.5 & 4.3 & \\
\hline Kiowa_262_3FIN2 & 119 & 0.68 & 0.1096 & 0.00075 & 3.188 & 0.04 & 0.2132 & 0.00305 & 0.88514 & 1456 & 9 & 1243 & 16.5 & 1785 & 12.5 & DISCARD & \#VALUE! & 30.4 & \\
\hline Kiowa_262_23.FIN2 & 275.3 & 0.54 & 0.0809 & 0.001 & 0.92 & 0.0165 & 0.0818 & 0.00085 & 0.5911 & 658 & 8.5 & 506 & 5 & 1206 & 24.5 & DISCARD & \#VALUE! & 23.1 & \\
\hline Kiowa_262_38.FIN2 & 145 & 0.50 & 0.0972 & 0.00095 & 1.94 & 0.07 & 0.145 & 0.0055 & 0.96568 & 1071 & 24.5 & 869 & 30.5 & 1566 & 19 & DISCARD & \#VALUE! & 44.5 & \\
\hline Kiowa_262_42.FIN2 & 206 & 0.38 & 0.0846 & 0.00095 & 1.181 & 0.0215 & 0.1016 & 0.0021 & 0.80281 & 786 & 10 & 622 & 12 & 1289 & 21.5 & DISCARD & \#VALUE! & 20.9 & \\
\hline Kiowa_262_47.FIN2 & 326 & 0.37 & 0.0949 & 0.00075 & 1.571 & 0.033 & 0.1178 & 0.0022 & 0.90648 & 949 & 12.5 & 717 & 12.5 & 1518 & 15 & DISCARD & \#VALUE! & 24.4 & \\
\hline Kiowa_262_56.FIN2 & 219.6 & 0.98 & 0.0951 & 0.00105 & 1.433 & 0.0325 & 0.1103 & 0.0029 & 0.90909 & 892 & 14 & 672 & 17 & 1514 & 20.5 & DISCARD & \#VALUE! & 24.7 & \\
\hline Kiowa_262_59.FIN2 & 627 & 0.39 & 0.1033 & 0.0007 & 2.762 & 0.0325 & 0.1902 & 0.00215 & 0.84314 & 1340 & 9.5 & 1121 & 12 & 1684 & 12.5 & DISCARD & \#VALUE! & 33.4 & \\
\hline Name: DB-D2-10 & & & & internal & & internal & & internal & & 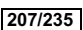 & internal & $206 / 238$ & internal & $207 / 206$ & internal & Best age & internal & & \\
\hline Analysis ID & {$[\mathrm{U}] \mathrm{ppm}$} & $\mathrm{Th} / \mathrm{U}$ & $207 / 206$ & $1 \sigma$ error & 207/235 & 10 error & 206/238 & 10 error & RHO & Age Ma & $1 \sigma$ error & Age (Ma) & $1 \sigma$ error & Age (Ma) & $1 \sigma$ error & (Ma) & $1 \sigma$ error & | \% Discordance ${ }^{*}$ & Rim/Core \\
\hline DB-D2-10_30.FIN2 & & 2.63 & 0.0501 & 0.00105 & 0.0606 & 0.00115 & 0.00884 & 0.000085 & 0.17853 & 59.7 & 1.1 & 56.7 & 0.55 & 180 & 39.5 & 56.7 & 0.6 & 5.0 & \\
\hline DB-D2-10_103.FII & 382 & 0.21 & 0.0483 & 0.00115 & 0.0648 & 0.0015 & 0.00966 & 0.00011 & 0.083987 & 6 & 1.4 & 62 & 0.7 & 112 & 45.5 & 62 & 0.7 & 2.5 & \\
\hline DB-D2-10_124.FIN2 & 90.4 & 0.81 & 0.054 & 0.0023 & 0. & 0.0033 & 0.00982 & 0.000165 & 0.25488 & 71 & 3.1 & 63 & 1.05 & 290 & 80 & 63 & 1.1 & 12.3 & \\
\hline DB-D2-10_35.FIN2 & 728 & 0.38 & 0.0491 & 0.001 & 0.06 & 0.001 & 0.00 & 0.0001 & -0.002916 & 65 & 1.25 & 63.1 & 0.65 & 137 & 37.5 & & 0.7 & 3.2 & \\
\hline DB-D2-10_62.FIN2 & 331 & 1.06 & 0.0506 & 0.00 & 0.0694 & 0.002 & 0.00 & 0.00008 & 0.05376 & 67 & 1.95 & 63.2 & 0.5 & 18 & 49 & 6 & 0.5 & 6.9 & \\
\hline DB-D2-10_126.FIN2 & 592 & 0.86 & 0.0517 & 0.0014 & 0.0692 & 0.001 & 0.009 & 0.000075 & 0.003354 & 67 & 1.45 & 63.68 & 0.48 & 218 & 48 & 63 & 0.5 & 6.1 & \\
\hline DB-D2-10_18.FIN2 & 355 & 0.81 & 0.054 & 0.00275 & 0.0751 & 0.0032 & 0.00999 & 0.00017 & -0.052125 & 73 & 3.05 & 64 & 1.1 & 320 & 105 & 64.1 & 1.1 & 12.7 & \\
\hline DB-D2-10_23.FIN2 & 89 & $\begin{array}{l}1.33 \\
1.35\end{array}$ & 0.0487 & $\begin{array}{r}0.0028 \\
0.0275\end{array}$ & 0.0654 & 0.00355 & 0.01006 & 0.00016 & 0.007706 & 65.2 & 3.6 & 64. & 1 & 60 & 90 & 64 & 1.0 & 1.1 & \\
\hline $\begin{array}{l}\text { DB-D2-10_80.FIN2 } \\
\text { DB-D2-10 50.FIN2 }\end{array}$ & 143.8 & 1.35 & $\begin{array}{r}0.053 \\
0.0528\end{array}$ & 0.00275 & 0.0736 & 0.0039 & 0.01005 & 0.00015 & 0.060008 & 71.7 & 3.55 & 64.5 & 0.95 & 270 & 90 & 64.5 & 1.0 & 10.0 & \\
\hline $\begin{array}{l}\text { DB-D2-10-50.FIN2 } \\
\text { DB-D2-10 139.FIN2 }\end{array}$ & $\begin{array}{r}192 \\
2564\end{array}$ & $\begin{array}{l}1.18 \\
0.92\end{array}$ & $\begin{array}{l}0.0528 \\
0.0493\end{array}$ & 0.00255 & 0.0721 & 0.00325 & 0.01009 & 0.000145 & -0.020709 & 70.2 & ${ }^{3}$ & 64.7 & 0.95 & 210 & 80 & 64.7 & 1.0 & 7.8 & \\
\hline $\begin{array}{l}\text { D-D2-10 }-139 \text {.FIN2 } \\
\text { DB-D2-10 43.FIN2 }\end{array}$ & $\begin{array}{r}256.4 \\
93.8\end{array}$ & $\begin{array}{l}0.92 \\
106\end{array}$ & $\begin{array}{l}0.0493 \\
0.0513\end{array}$ & $\begin{array}{l}0.00115 \\
0.00275\end{array}$ & $\begin{array}{l}0.0689 \\
0.0698\end{array}$ & $\begin{array}{l}0.00165 \\
0.00355\end{array}$ & $\begin{array}{r}0.01009 \\
0.0101\end{array}$ & $\begin{array}{r}0.00009 \\
0.000185\end{array}$ & $\begin{array}{r}0.10869 \\
-0\end{array}$ & 67.5 & $\begin{array}{r}1.55 \\
33\end{array}$ & 64.7 & 0.6 & 141 & ${ }_{85}^{44}$ & 64.7 & 0.6 & 4.1 & \\
\hline $\begin{array}{l}\text { DB-D2-10_-43.FIN2 } \\
\text { DB-D2-10 5.FIN2 }\end{array}$ & 93.8 & $\begin{array}{l}1.06 \\
1.37\end{array}$ & & $\begin{array}{l}0.00275 \\
0.00165\end{array}$ & 0.0698 & $\begin{array}{l}0.00355 \\
0.00215\end{array}$ & 0.0101 & $\begin{array}{l}0.000185 \\
0.000105\end{array}$ & -0.024447 & 68 & 3.3 & 64.8 & 1.2 & 140 & 85 & 64.8 & 1.2 & 4.7 & \\
\hline $\begin{array}{l}\text { DB-D2-10-5.FIN2 } \\
\text { DB-D2-10 60.FIN2 }\end{array}$ & $\begin{array}{l}228 \\
258\end{array}$ & 0.24 & $\begin{array}{l}0.0523 \\
0.0513\end{array}$ & $\begin{array}{l}0.00165 \\
0.0019\end{array}$ & 0.0712 & $\begin{array}{r}0.00215 \\
0.0028\end{array}$ & 0.01014 & $\begin{array}{l}0.000105 \\
0.00015\end{array}$ & $\begin{array}{l}0.11813 \\
0.23711\end{array}$ & $\begin{array}{l}0.4 \\
69.6\end{array}$ & 26 & $\begin{array}{r}64.9 \\
65\end{array}$ & $\begin{array}{l}0.05 \\
0.95\end{array}$ & 210 & $\begin{array}{l}55 \\
70\end{array}-10$ & $\begin{array}{l}64.9 \\
65.0\end{array}$ & $\begin{array}{l}0.1 \\
1.0\end{array}$ & $\begin{array}{l}1.8 \\
6.6\end{array}$ & \\
\hline DB-D2-10_86.FIN2 & 389.2 & 1.98 & 0.05 & 0.00115 & 0.0705 & 0.0016 & 0.01013 & 0.00009 & 0.24695 & 69 & 1.5 & 65 & 0.6 & 193 & 45 & 65.0 & 0.6 & 5.8 & \\
\hline DB-D2-10_87.FIN2 & 92 & 1.20 & 0.0529 & 0.0031 & 0.0748 & 0.00465 & 0.01017 & 0.00026 & 0.017382 & 72.8 & 4.35 & 65.2 & 1.65 & 250 & 105 & 65.2 & 1.7 & 10.4 & \\
\hline DB-D2-10_65.FIN2 & 222.8 & 0.37 & 0.0566 & 0.00245 & 0.0769 & 0.00275 & 0.01027 & 0.0001 & 0.083097 & 74.9 & 2.55 & 65.9 & 0.65 & 340 & 70 & 65.9 & 0.7 & 12.0 & \\
\hline DB-D2-10_19.FIN2 & 436.7 & 0.41 & 0.0511 & 0.00125 & 0.0728 & 0.0018 & 0.01032 & 0.000085 & 0.090137 & 71.2 & 1.65 & 66.2 & 0.55 & 209 & 46.5 & 66.2 & 0.6 & 7.0 & \\
\hline DB-D2-10_108.FIN2 & 63.2 & 0.72 & 0.0495 & 0.00365 & 0.072 & 0.0055 & 0.01037 & 0.000215 & -0.002719 & 70 & 5 & 66.5 & 1.4 & 160 & 130 & 66.5 & 1.4 & 5.0 & \\
\hline DB-D2-10_134.FIN2 & 271.3 & 0.23 & 0.0483 & 0.00135 & 0.0687 & 0.0017 & 0.0104 & 0.000095 & -0.069606 & 67.3 & 1.65 & 66.7 & 0.6 & 100 & 50 & 66.7 & 0.6 & 0.9 & \\
\hline
\end{tabular}




\begin{tabular}{|c|c|c|c|c|c|c|c|c|c|c|}
\hline DB-D2-10_2.FIN2 & 428 & 1.27 & 0.0523 & 0.0011 & 0.0747 & 0.0015 & 0.01042 & 0.000075 & 0.02187 & 73 \\
\hline DB-D2-10_20.FIN2 & 197.1 & 0.93 & 0.0503 & 0.00205 & 0.071 & 0.00275 & 0.01043 & 0.00016 & 0.12079 & 69.3 \\
\hline DB-D2-10_4.FIN2 & 39.51 & 1.54 & 0.055 & 0.006 & 0.075 & 0.0065 & 0.01048 & 0.000185 & 0.050714 & 71 \\
\hline DB-D2-10_76.FIN2 & 398 & 0.37 & 0.0525 & 0.00485 & 0.078 & 0.0075 & 0.01065 & 0.000145 & -0.26927 & 75 \\
\hline DB-D2-10_138.FIN2 & 489 & 0.65 & 0.0497 & 0.0008 & 0.1003 & 0.00195 & 0.0144 & 0.00011 & 0.45247 & 96.8 \\
\hline DB-D2-10_33.FIN2 & 1118 & 0.88 & 0.0489 & 0.0005 & 0.1048 & 0.00115 & 0.01541 & 0.00008 & 0.027294 & 101.1 \\
\hline DB-D2-10_131.FIN2 & $\begin{array}{l}544 \\
185\end{array}$ & 0.42 & 0.0532 & 0.0013 & 0.1186 & 0.0028 & 0.01613 & 0.000135 & 0.093968 & 113.4 \\
\hline DB-D2-10_95.FIN2 & $\begin{array}{r}185 \\
320\end{array}$ & 1.02 & 0.0577 & $\begin{array}{r}0.0016 \\
0.0125\end{array}$ & 0.199 & 0.005 & 0.02507 & 0.00027 & 0.033898 & 184.5 \\
\hline DB-D2-10_93.FIN2r & $\begin{array}{l}322.2 \\
1152\end{array}$ & 0.35 & 0.0526 & 0.00135 & 0.2091 & 0.00485 & 0.02829 & $\begin{array}{r}0.00036 \\
0.00265\end{array}$ & 0.076193 & $\begin{array}{l}192.5 \\
307.8\end{array}$ \\
\hline DB-D2-10_123.FIN2 & 1152 & 2.17 & 0.0559 & 0.00055 & 0.3549 & 0.00355 & 0.04609 & 0.000265 & 0.21553 & 307.8 \\
\hline DB-D2-10_92.FIN2 & 477 & 1.04 & 0.0632 & 0.00055 & 0.556 & 0.005 & 0.0632 & 0.00033 & 0.48293 & 448.5 \\
\hline DB-D2-10_110.FIN2 & 78.3 & 0.57 & 0.0611 & 0.0015 & 0.553 & 0.013 & 0.0658 & 0.0005 & 0.10259 & 442 \\
\hline DB-D2-10_127.FIN2r & 311 & 0.08 & 0.0598 & 0.002 & 0.549 & 0.018 & 0.0671 & 0.00135 & 0.036468 & 442 \\
\hline DB-D2-10_31.FIN2c & 6.01 & 0.37 & 0.0768 & 0.00455 & 1.96 & 0.125 & 0.1822 & 0.0049 & 0.43471 & 1069 \\
\hline DB-D2-10_119.FIN2 & 49.8 & 0.84 & 0.0758 & 0.00105 & 1.914 & 0.0265 & 0.1816 & 0.00165 & 0.29767 & 1082 \\
\hline DB-D2-10_137.FIN2 & 108.7 & 0.83 & 0.0756 & 0.00055 & 1.894 & 0.0145 & 0.1813 & 0.001 & 0.31989 & 1077 \\
\hline DB-D2-10_105.FIN2 & 67 & 1.44 & 0.0758 & 0.00085 & 1.895 & 0.022 & 0.181 & 0.00155 & 0.39043 & 1076 \\
\hline DB-D2-10_52.FIN2 & 191 & 0.94 & 0.07594 & 0.00047 & 1.838 & 0.0125 & 0.1762 & 0.001 & 0.42144 & 1057.5 \\
\hline DB-D2-10_91.FIN2 & 67.9 & 0.94 & 0.0761 & 0.0008 & 1.931 & 0.0195 & 0.1829 & 0.0012 & 0.27304 & 1090 \\
\hline DB-D2-10_104.FIN2 & 232 & 0.92 & 0.0761 & 0.00065 & 1.958 & 0.0175 & 0.185 & 0.0011 & 0.29462 & 1099 \\
\hline DB-D2-10_128.FIN2 & 167.4 & 0.47 & 0.076 & 0.0009 & 1.627 & 0.018 & 0.155 & 0.0014 & 0.009182 & 980 \\
\hline DB-D2-10_102.FIN2 & 142 & 0.70 & 0.0759 & 0.00065 & 1.908 & 0.0145 & 0.1814 & 0.00105 & 0.21305 & 1084 \\
\hline DB-D2-10_97.FIN2 & 249 & 1.26 & 0.0764 & 0.00055 & 2.012 & 0.0155 & 0.1895 & 0.00095 & 0.35797 & 1118 \\
\hline DB-D2-10_99.FIN2 & 246 & 0.57 & 0.07659 & 0.000425 & 1.965 & 0.011 & 0.1847 & 0.00085 & 0.43866 & 1102.5 \\
\hline DB-D2-10_8.FIN2 & 76.6 & 1.45 & 0.0769 & 0.00075 & 1.994 & 0.0185 & 0.1899 & 0.00125 & 0.32189 & 1111 \\
\hline DB-D2-10_84.FIN2 & 152.9 & 1.01 & 0.0771 & 0.0006 & 1.775 & 0.013 & 0.1659 & 0.00095 & 0.23002 & 1034.8 \\
\hline DB-D2-10_36.FIN2 & 231 & 0.61 & 0.07673 & 0.00049 & 1.977 & 0.0135 & 0.186 & 0.0011 & 0.36949 & 1106.3 \\
\hline DB-D2-10_12.FIN2 & 209 & 1.03 & 0.077 & 0.00055 & 1.973 & 0.0145 & 0.1867 & 0.00095 & 0.39922 & 1104.3 \\
\hline DB-D2-10_38.FIN2 & 229.5 & 0.68 & 0.0771 & 0.00065 & 1.833 & 0.018 & 0.1719 & 0.00145 & 0.58759 & 1056 \\
\hline DB-D2-10_122.FIN2 & 63.3 & 0.93 & 0.078 & 0.0011 & 1.95 & 0.025 & 0.1823 & 0.0015 & 0.10887 & 1094 \\
\hline DB-D2-10_28.FIN2 & 91.2 & 0.93 & 0.0777 & 0.00085 & 1.947 & 0.023 & 0.1812 & 0.00095 & 0.15334 & 1093 \\
\hline DB-D2-10_14.FIN2 & 53.1 & 1.20 & 0.0775 & 0.00095 & 1.956 & 0.0255 & 0.1839 & 0.0011 & 0.26877 & 1096 \\
\hline DB-D2-10_114.FIN2 & $\begin{array}{l}145.5 \\
166.4\end{array}$ & 0.81 & 0.0776 & 0.00065 & 2.015 & 0.0165 & 0.1882 & 0.00095 & 0.11965 & 1118 \\
\hline DB-D2-10_106.FIN2 & 166.4 & 0.92 & $\begin{array}{l}0.0775 \\
0.079\end{array}$ & 0.00055 & $\begin{array}{l}1.966 \\
2012\end{array}$ & 0.0155 & 0.1834 & $\begin{array}{r}0.0012 \\
0.0135\end{array}$ & 0.47164 & 1102 \\
\hline $\begin{array}{l}\text { DB-D2-10-135.FIN2 } \\
\text { D-D2-10 17 FIN2 }\end{array}$ & $\begin{array}{r}133 \\
99\end{array}$ & $\begin{array}{l}1.17 \\
0.58\end{array}$ & $\begin{array}{l}0.0789 \\
0.0778\end{array}$ & $\begin{array}{r}0.0012 \\
0.00065\end{array}$ & $\begin{array}{l}2.012 \\
1.969\end{array}$ & $\begin{array}{l}0.0265 \\
0.0175\end{array}$ & $\begin{array}{l}0.1866 \\
0.1837\end{array}$ & 0.00135 & $\begin{array}{r}0.1219 \\
0.92196\end{array}$ & 1114 \\
\hline $\begin{array}{l}\text { DB-D2-10-17.FIN2 } \\
\text { DB-D2-10 37.FIN2 }\end{array}$ & $\begin{array}{r}99 \\
131.9\end{array}$ & 0.72 & $\begin{array}{l}0.0778 \\
0.0774\end{array}$ & $\begin{array}{l}0.00065 \\
0.0006\end{array}$ & $\begin{array}{l}1.969 \\
1.986\end{array}$ & $\begin{array}{l}0.0175 \\
0.016\end{array}$ & $\begin{array}{l}0.1837 \\
0.1844\end{array}$ & $\begin{array}{r}0.001 \\
0.00075\end{array}$ & 0.29196 & 1102 \\
\hline DB-D2-10_100.FIN2 & 158.1 & 0.80 & 0.0777 & 0.00065 & $\begin{array}{l}1.900 \\
1.982\end{array}$ & $\begin{array}{l}0.0165 \\
0.016\end{array}$ & $\begin{array}{l}0.1838 \\
0.1838\end{array}$ & 0.0011 & 0.32202 & $\begin{array}{l}1109 \\
1107\end{array}$ \\
\hline DB-D2-10_59.FIN2 & 93.5 & 1.13 & 0.0783 & 0.0009 & 1.981 & 0.0245 & 0.184 & 0.0012 & 0.34531 & 1104 \\
\hline DB-D2-10_89.FIN2 & 57.1 & 0.74 & 0.08 & 0.00165 & 1.99 & 0.0385 & 0.182 & 0.0022 & 0.12246 & 1101 \\
\hline DB-D2-10_57.FIN2 & 85.8 & 0.57 & 0.0782 & 0.0007 & 1.97 & 0.0215 & 0.184 & 0.0014 & 0.56123 & 1102 \\
\hline DB-D2-10_10.FIN2 & 114.8 & 0.82 & 0.079 & 0.00085 & 1.867 & 0.0205 & 0.1729 & 0.00175 & 0.41016 & 1068 \\
\hline DB-D2-10_130.FIN2 & 145 & 0.70 & 0.0789 & 0.00075 & 1.979 & 0.018 & 0.1822 & 0.00115 & 0.043188 & 1106 \\
\hline DB-D2-10_42.FIN2 & 404 & 0.68 & 0.07866 & 0.000475 & 1.775 & 0.0115 & 0.1636 & 0.0009 & 0.35437 & 1035.2 \\
\hline DB-D2-10_121.FIN2 & 101.9 & 0.62 & 0.0787 & 0.0007 & 2.009 & 0.0205 & 0.1845 & 0.00115 & 0.44141 & 1117 \\
\hline DB-D2-10_61.FIN2 & 58.91 & 0.95 & 0.08 & 0.00115 & 2.088 & 0.0335 & 0.1886 & 0.0015 & 0.31117 & 1137 \\
\hline DB-D2-10_21.FIN2 & 137 & 0.91 & 0.0792 & 0.0008 & 1.986 & 0.019 & 0.1816 & 0.00095 & 0.15786 & 1109 \\
\hline DB-D2-10_39.FIN2 & 242.2 & 0.95 & 0.0794 & 0.000485 & 2.002 & 0.0125 & 0.1828 & 0.0009 & 0.44312 & 1114.9 \\
\hline DB-D2-10_82.FIN2 & 68.9 & 0.81 & 0.0808 & 0.00115 & 1.999 & 0.027 & 0.1789 & 0.00115 & 0.062605 & 1109 \\
\hline DB-D2-10_24.FIN2r & 206.9 & 0.28 & 0.0799 & 0.00115 & 1.588 & 0.026 & 0.1438 & 0.0012 & 0.22716 & 963 \\
\hline DB-D2-10_113.FIN2 & 284.4 & 0.46 & 0.0806 & 0.00065 & 2.006 & 0.018 & 0.1803 & 0.00095 & 0.35676 & 1115 \\
\hline DB-D2-10_125.FIN2 & 54.5 & 1.07 & 0.0833 & 0.00205 & 2.08 & & 0.1824 & 0.001 & 0.075365 & 1131 \\
\hline DB-D2-10_69.FIN2 & 152.5 & 0.54 & 0.0809 & 0.000 & 2.159 & 0.0155 & 0.1932 & 0.0012 & 0.30116 & 1166 \\
\hline DB-D2-10_81.FIN2 & 82.8 & 0.66 & 0.0833 & 0.0 & 2.098 & 0.032 & 0.1822 & 0.0013 & 0.050367 & 1145 \\
\hline DB-D2-10_72.FIN2 & 85 & 2.71 & 0.0852 & 0.00175 & 1.92 & 0.03 & 0.1 & 0.002 & 0.11315 & 1075 \\
\hline DB-D2-10_75.FIN2 & 99.2 & 0.45 & 0.084 & 0.000 & 2.32 & 0.0275 & 0.1985 & 0.00115 & 0.15972 & 1214 \\
\hline DB-D2-10_64.FIN2 & 24.51 & 1.16 & 0.0842 & 0.00135 & 2.573 & 0.0365 & 0.2233 & 0.00215 & 0.016212 & 1290 \\
\hline DB-D2-10_24.FIN2c & 284 & 0.61 & 0.0844 & 0.0008 & 2.556 & 0.03 & 0.2193 & 0.00205 & 0.64244 & 1286 \\
\hline $\begin{array}{l}\text { DB-D2-10_16.FIN2 } \\
\text { DB-D2-10 116 FIN2 }\end{array}$ & $\begin{array}{r}236 \\
236\end{array}$ & 0.94 & 0.0861 & 0.00065 & $\begin{array}{l}1.868 \\
2244\end{array}$ & $\begin{array}{l}0.0175 \\
0.044\end{array}$ & 0.1584 & 0.00125 & 0.59776 & 1068 \\
\hline $\begin{array}{l}\text { DB-D2-10-116.FIN2 } \\
\text { D-D2-10 47 FIN2 }\end{array}$ & $\begin{aligned} 146.2 \\
215\end{aligned}$ & 0.77 & 0.0889 & 0.00115 & 2.244 & $\begin{array}{r}0.044 \\
0.0175\end{array}$ & 0.1854 & 0.00265 & 0.70138 & 1191 \\
\hline $\begin{array}{l}\text { DB-D2-10-47.FIN2 } \\
\text { DB-D2-10 55.FIN2C }\end{array}$ & $\begin{array}{l}215 \\
301\end{array}$ & $\begin{array}{l}2.05 \\
0.13\end{array}$ & $\begin{array}{r}0.0895 \\
0.09\end{array}$ & $\begin{array}{l}0.0005 \\
0.00065\end{array}$ & $\begin{array}{l}3.128 \\
2.549\end{array}$ & $\begin{array}{l}0.0175 \\
0.0425\end{array}$ & $\begin{array}{l}0.2539 \\
0.2063\end{array}$ & $\begin{array}{r}0.0012 \\
0.003\end{array}$ & $\begin{array}{l}0.35711 \\
0.89301\end{array}$ & $\begin{array}{r}1438.5 \\
1281\end{array}$ \\
\hline DB-D2-10_58.FIN2 & 121.1 & 0.53 & 0.0901 & 0.00065 & 3.062 & 0.0245 & 0.2462 & 0.00165 & 0.54154 & 1423 \\
\hline DB-D2-10_132.FIN2 & 117.3 & 0.65 & 0.0901 & 0.00085 & 2.964 & 0.028 & 0.2383 & 0.0019 & 0.30098 & 1395 \\
\hline DB-D2-10_120.FIN2 & 67.2 & 1.76 & 0.0903 & 0.00075 & 3.129 & 0.0235 & 0.2519 & 0.0015 & 0.15151 & 1437 \\
\hline DB-D2-10_-90.FIN2 & 150 & 1.05 & 0.0902 & 0.00055 & 3.085 & 0.0195 & 0.2464 & 0.0013 & 0.48828 & 1427.1 \\
\hline DB-D2-10_71.FIN2 & 125.6 & 0.50 & 0.0909 & 0.00115 & 2.12 & 0.06 & 0.1688 & 0.0044 & 0.91277 & 1140 \\
\hline DB-D2-10_70.FIN2 & 791 & 0.70 & 0.09051 & 0.00049 & 3.136 & 0.0205 & 0.2496 & 0.0016 & 0.60832 & 1440 \\
\hline DB-D2-10_53.FIN2 & 221.1 & 0.80 & 0.0907 & 0.000 & 3.05 & 0.0215 & 0.2434 & 0.00125 & 0.54609 & 1421 \\
\hline DB-D2-10_-85.FIN2 & 282 & 0.90 & 0.09096 & 0.00 & 2.928 & 0.018 & 0.2313 & 0.0013 & 0.58157 & 1388.9 \\
\hline DB-D2-10_140.FIN2 & 221 & 1.03 & 0.0914 & 0.00 & 2.832 & 0.02 & 0.224 & 0.0017 & 0.6394 & 1363 \\
\hline DB-D2-10_77.FIN2 & 153.1 & 0.82 & 0.0917 & & 3.146 & 0.0295 & 0.2466 & 0.00155 & 0.42 & 1442 \\
\hline DB-D2-10_109.FIN2 & 171 & 0.80 & 0.0921 & 0.0 & 3.23 & 0.01 & 0.2546 & 0.0013 & 0.26447 & 1463.3 \\
\hline DB-D2-10_54.FIN2 & 168.9 & 0.58 & 0.0926 & 0.0007 & 2.575 & & 0.2022 & 0.00125 & 0.44595 & 1291 \\
\hline DB-D2-10_101.FIN2 & 167 & 0.69 & 0.0926 & 0.00065 & 3.11 & 0.025 & 0.2416 & 0.0014 & 0.44744 & 1433 \\
\hline DB-D2-10_112.FIN2 & 256.7 & 1.13 & 0.0928 & 0.0006 & 2.9 & 0.023 & 0.2269 & 0.00145 & 0.60245 & 1379 \\
\hline
\end{tabular}

\begin{tabular}{|c|c|c|c|}
\hline 1.4 & 66.85 & 0.495 & $25 \mathrm{~s}$ \\
\hline 2.6 & 66.9 & 1 & 17 \\
\hline 5.5 & 67.2 & 1.15 & \\
\hline 6.5 & 68.3 & 0.95 & 12 \\
\hline 1.8 & 92.2 & $\begin{array}{l}0.7 \\
05\end{array}$ & 17 \\
\hline 1.05 & $\begin{array}{r}98.6 \\
\end{array}$ & $\begin{array}{r}0.5 \\
0.5\end{array}$ & 14 \\
\hline 2.5 & 103.1 & $\begin{array}{r}0.85 \\
17\end{array}$ & \\
\hline 4.55 & $\begin{array}{l}159.6 \\
1708\end{array}$ & $\begin{array}{r}1.7 \\
225\end{array}$ & \\
\hline 4.05 & $\begin{array}{l}179.8 \\
2004\end{array}$ & $\begin{array}{r}2.25 \\
16\end{array}$ & $\begin{array}{l}299 \\
42\end{array}$ \\
\hline $\begin{array}{l}2.6 \\
3.3\end{array}$ & $\begin{array}{r}290.4 \\
395\end{array}$ & $\begin{array}{r}1.6 \\
2\end{array}$ & $\begin{array}{l}42 \\
71\end{array}$ \\
\hline $\begin{array}{r}3.3 \\
8\end{array}$ & $\begin{array}{r}395 \\
410.4\end{array}$ & 3.15 & 55 \\
\hline 11.5 & 418 & $\begin{array}{r}8.10 \\
8.5\end{array}$ & 5 \\
\hline 43 & 1077 & 26.5 & \\
\hline 9.5 & 1075 & 9 & 1055 \\
\hline 5 & 1074 & 5.5 & 1072 \\
\hline 7.5 & 1072 & 8.5 & 107. \\
\hline 4.45 & 1046 & 5.5 & 1086 \\
\hline & 1082 & 6.5 & 1086 \\
\hline 6 & 1094 & 6 & $\begin{array}{l}1086 \\
108\end{array}$ \\
\hline $\begin{array}{l}7 \\
5\end{array}$ & $\begin{array}{r}929 \\
1074\end{array}$ & $\begin{array}{r}8 \\
5.5\end{array}$ & $\begin{array}{l}108 \\
1096\end{array}$ \\
\hline 5 & 1118 & 5 & 1100 \\
\hline 3.7 & 1092.3 & 4.7 & 1107 \\
\hline 6.5 & 1121 & 7 & $110 \mathrm{~s}$ \\
\hline 4.75 & 991 & 5 & 111 \\
\hline 4.55 & 1099 & 6 & 111 \\
\hline 4.95 & 1103 & 5.5 & 1116 \\
\hline 6.5 & 1022 & 8 & 1116 \\
\hline 8.5 & 1079 & 8 & 1116 \\
\hline 7.5 & 1073 & 5.5 & 1117 \\
\hline 8.5 & 1088 & 6 & 1119 \\
\hline 5.5 & 1112 & 5 & 112 \\
\hline 5.5 & 1085 & 6.5 & 1125 \\
\hline 8 & 1104 & 7 & 1125 \\
\hline 6 & 1087 & 5.5 & 1127 \\
\hline 5.5 & 1090.7 & 4.2 & $112 \varepsilon$ \\
\hline 5.5 & 1087 & 6 & $112 \mathrm{~S}$ \\
\hline 8 & 1088 & 6.5 & 1132 \\
\hline $\begin{array}{r}12.5 \\
7.5\end{array}$ & $\begin{array}{l}1076 \\
1088\end{array}$ & $\begin{array}{r}12 \\
7.5\end{array}$ & $\begin{array}{l}1136 \\
1144\end{array}$ \\
\hline 7.5 & 1027 & 9.5 & 115 \\
\hline & 1078 & 6 & 115 \\
\hline 4.1 & 976.5 & 4.95 & 115 \\
\hline 6.5 & 1091 & 6 & 15 \\
\hline 10.5 & 1113 & 8 & 16 \\
\hline & 1076 & 5 & 17 \\
\hline 4.25 & 1082 & 5 & 17 \\
\hline 9 & 1061 & 6.5 & \\
\hline 10 & 866 & 7 & \\
\hline & 1068 & 5 & \\
\hline 12.5 & 1079 & 5.5 & \\
\hline 5 & 1138 & 6.5 & \\
\hline 10.5 & 1078 & 7 & \\
\hline 13 & 979 & 11 & \\
\hline & 1167 & 6 & \\
\hline 10.5 & 1298 & 11.5 & \\
\hline 8.5 & 1278 & 11 & \\
\hline $\begin{array}{r}6 \\
135\end{array}$ & $\begin{array}{r}947 \\
1096\end{array}$ & 145 & 33 \\
\hline 13.5 & $\begin{array}{l}1096 \\
1458\end{array}$ & 14.5 & \\
\hline 4.25 & 1458 & 6 & \\
\hline 13 & $\begin{array}{l}1206 \\
1418\end{array}$ & $\begin{array}{r}16 \\
85\end{array}$ & \\
\hline 6 & $\begin{array}{l}1418 \\
1377\end{array}$ & 8.5 & \\
\hline 1 & 131 & 10 & \\
\hline 49 & $\begin{array}{l}1440 \\
1419\end{array}$ & $\begin{array}{l}1.5 \\
65\end{array}$ & \\
\hline $\begin{array}{l}4.9 \\
20\end{array}$ & 1001 & $\begin{array}{l}0.5 \\
24.5\end{array}$ & \\
\hline 5 & 1436 & $\begin{array}{r}24.5 \\
8\end{array}$ & 43 \\
\hline 5.5 & 1404 & 6.5 & 143 \\
\hline 4.75 & 1341 & 6.5 & \\
\hline 5.5 & 1302 & 9 & \\
\hline 7 & 1420 & 8 & \\
\hline 4.65 & 1462 & 6.5 & \\
\hline 6 & 1187 & 6.5 & \\
\hline 6 & 1394 & 7.5 & \\
\hline 6 & 1318 & & $147 \pi$ \\
\hline
\end{tabular}

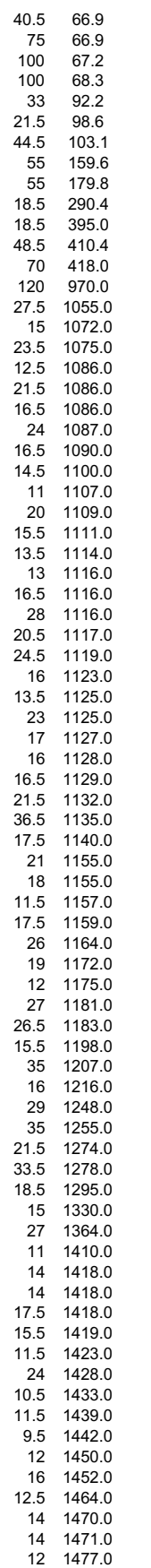

$\begin{array}{ccc} & & \\ 0.5 & 8.4 & \\ 1.0 & 3.5 & \\ 1.2 & 5.4 & \\ 1.0 & 8.9 & \\ 0.7 & 4.8 & \\ 0.5 & 2.5 & \\ 0.9 & 9.1 & \\ 1.7 & 13.5 & \\ 2.3 & 6.6 & \text { Rim } \\ 1.6 & 5.7 & \\ 2.0 & 11.9 & \\ 3.2 & 7.1 & \\ 8.5 & 5.4 & \text { Rim } \\ 120.0 & -11.0 & \text { Core } \\ 27.5 & -1.9 & \\ 15.0 & -0.2 & \\ 23.5 & 0.3 & \\ 12.5 & 3.7 & \\ 21.5 & 0.4 & \\ 16.5 & -0.7 & \\ 24.0 & 14.5 & \\ 16.5 & 1.5 & \\ 14.5 & -1.6 & \\ 11.0 & 1.3 & \\ 20.0 & -1.1 & \\ 15.5 & 10.8 & \\ 13.5 & 1.3 & \\ 13.0 & 1.2 & \\ 16.5 & 8.4 & \\ 28.0 & 3.3 & \\ 20.5 & 3.9 & \\ 24.5 & 2.8 & \\ 16.0 & 1.0 & \\ 13.5 & 3.6 & \\ 23.0 & 1.9 & \\ 17.0 & 3.5 & \\ 16.0 & 3.3 & \\ 16.5 & 3.7 & \\ 21.5 & 3.9 & \\ 36.5 & 5.2 & \\ 17.5 & 4.6 & \\ 21.0 & 11.1 & \\ 18.0 & 6.7 & \\ 11.5 & 15.6 & \\ 17.5 & 5.9 & \\ 26.0 & 4.4 & \\ 19.0 & 8.2 & \\ 12.0 & 7.9 & \\ 27.0 & 10.2 & \\ 26.5 & 26.8 & \\ 15.5 & 10.9 & \\ 35.0 & 10.6 & \\ 16.0 & 6.4 & \\ 29.0 & 13.6 & \\ 35.0 & 22.0 & \\ 21.5 & 8.4 & \\ 33.5 & -1.6 & \\ 18.5 & 1.3 & \\ 15.0 & 28.8 & \\ 27.0 & 19.6 & \\ 11.0 & -3.4 & \\ 14.0 & 15.0 & \\ 14.0 & 0.0 & \\ 17.5 & 2.9 & \\ 15.5 & -2.0 & \\ 11.5 & 0.3 & \\ 24.0 & 29.9 & \\ 10.5 & -0.2 & \\ 11.5 & 2.4 & \\ 9.5 & 7.0 & \\ 12.0 & 10.2 & \\ 16.0 & 2.2 & \\ 12.5 & 0.1 & \\ 14.0 & 19.3 & \\ 14.0 & 5.2 & \\ 12.0 & 10.8 & \\ & & \end{array}$


DB-D2-10_3.FIN2 DB-D2-10-83.FIN2 DB-D2-10-68.FIN2 DB-D2-10_79.FIN2 DB-D2-10_9.FIN2 DB-D2-10-11.FIN2 DB-D2-10-51.FIN2
DB-D2-10-94.FIN2 DB-D2-10-44.FIN2

DB-D2-10_46.FIN2

DBD2-10

DB-D2-10-96. FIN2

DB-D2-10-73.FIN2

DB-D2-10-22.FIN2C

DB-D2-10_118.FIN2

DB-D2-10-98.FIN2

DB-D2-10-7.FIN2

$210-26$ FN

D2 $10-31 . \mathrm{FIN}$

DB-D2-10-40.FIN2

DB-D2-10-19.FIN2

DB-D2-10-56.FIN2

DB-D2-10_66.FIN2

DB 22108 FIN

B-D2-10-107.FIN2

DBD2 10-115.FIN2

DB-D2-10-117.FIN2

DB-D2-10_136.FIN2

Sample Name: Kiowa-848

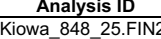

Kiowa_848_74.FIN2

848 24.FIN

Kiowa_848-51.FIN2

Kiowa_848_57.FIN2

Kiowa_848_81.FIN2

Iowa_848_80.FIN2

Kiowa_848_26.FIN2

Kiowa_848_12.FIN2

Kiowa_848_33.FIN2
Kiowa 848 36. FIN2

Kiowa_848_39.FIN2

Kiowa_848-10.FIN2

Kiowa_848_3.FIN2

Kiowa_848_82.FIN2

Kiowa_848_61.FIN2

Kiowa_848_19.FIN2

Kiowa_848_16.FIN2

$\begin{array}{rrrr}92 & 0.98 & 0.0935 & 0.00095 \\ 154.5 & 0.73 & 0.0933 & 0.0007 \\ 726 & 0.52 & 0.09315 & 0.0000415 \\ 178 & 0.78 & 0.0934 & 0.00065 \\ 176.1 & 0.99 & 0.0398 & 0.00085 \\ 208.2 & 0.19 & 0.0939 & 0.00055 \\ 234.2 & 0.39 & 0.0944 & 0.0005 \\ 285 & 0.14 & 0.0973 & 0.00075 \\ 321 & 0.32 & 0.0985 & 0.0008 \\ 132.9 & 1.19 & 0.0994 & 0.0006 \\ 525 & 0.07 & 0.1004 & 0.00065 \\ 508 & 0.28 & 0.1031 & 0.0008 \\ 59.5 & 0.63 & 0.1035 & 0.0011 \\ 1138 & 0.15 & 0.1059 & 0.00055 \\ 96 & 0.95 & 0.1067 & 0.0011 \\ 195.5 & 1.05 & 0.1066 & 0.00075 \\ 141.9 & 0.67 & 0.1066 & 0.00085 \\ 84.4 & 0.79 & 0.1085 & 0.00205 \\ 209.2 & 0.45 & 0.1084 & 0.00055 \\ 156 & 0.71 & 0.1095 & 0.0007 \\ 201.3 & 0.44 & 0.1999 & 0.00065 \\ 481 & 0.14 & 0.1144 & 0.0007 \\ 269.3 & 0.53 & 0.1151 & 0.00075 \\ 606 & 0.06 & 0.1175 & 0.00125 \\ 109.8 & 1.03 & 0.1177 & 0.0007 \\ 319 & 0.19 & 0.1281 & 0.00085 \\ 203.7 & 0.66 & 0.1663 & 0.00085 \\ 95.6 & 1.08 & 0.082 & 0.008 \\ 268 & 1.02 & 0.0599 & 0.0031 \\ 141 & 0.64 & 0.059 & 0.0055 \\ 122.2 & 1.33 & 0.074 & 0.0095 \\ 132 & 0.52 & 0.103 & 0.007 \\ 86.8 & 0.46 & 0.076 & 0.0115 \\ 338.5 & 0.52 & 0.0799 & 0.00305 \\ 164 & 1.40 & 0.0702 & 0.00415 \\ 178.2 & 0.966 & 0.062 & 0.0055 \\ 125 & 0.96 & 0.18 & 0.055 \\ 76.9 & 1.04 & 0.0596 & 0.00355 \\ 68.7 & 1.05 & 0.0891 & 0.0075 \\ 88.8 & 1.06 & 0.114 & 0.011 \\ 97.9 & 0.066 & 0.056 & 0.005 \\ 378 & 3.94 & 0.1205 & 0.00105 \\ 196.7 & 2.87 & 0.1082 & 0.0013 \\ 146 & 0.45 & 0.164 & 0.025 \\ 51.5 & 0.91 & 0.11 & 0.013 \\ 88 & 0.98 & 0.079 & 0.01 \\ 143.6 & 1.18 & 0.073 & 0.005 \\ & & & \end{array}$

\begin{tabular}{|c|c|c|c|c|c|}
\hline & 0.0285 & 0.2448 & 0.0015 & 0.099732 & \\
\hline & 0.026 & 0.2373 & 0.00145 & 0.20953 & \\
\hline 311 & 0.0215 & 0.2188 & 0.0016 & 0.81192 & \\
\hline 326 & 0.0235 & 2568 & 0.00105 & 23848 & \\
\hline 3.494 & 0.0335 & 2676 & 0.0016 & 25578 & \\
\hline 3.15 & 0.025 & 0.2446 & 0.00135 & 0.79853 & \\
\hline 3.278 & 0.019 & 0.2535 & 0.00115 & 0.48731 & 1475.8 \\
\hline 3.19 & 0.065 & 0.2356 & 0.0039 & 0.91077 & \\
\hline 3.768 & 0.0485 & ).2772 & 0.0029 & 0.79183 & 1582 \\
\hline 3.93 & 0.023 & .2865 & 0.0014 & 0.30051 & 1618.2 \\
\hline 3.561 & 0.028 & 0.2566 & 0.0017 & 0.65098 & \\
\hline 3.58 & 0.06 & 0.249 & 0.00335 & 0.90165 & 1534 \\
\hline 3.208 & 0.0405 & & 0.0023 & 0.54532 & \\
\hline 4.079 & 0.0325 & 0.2772 & 0.002 & 79783 & \\
\hline 4.376 & & & 0.00165 & & \\
\hline 4.521 & 0.0435 & & & & \\
\hline 4.549 & & & & & \\
\hline 3.49 & & & & & \\
\hline 4.697 & & & & & \\
\hline 3.844 & 0.0345 & & & & \\
\hline 4.495 & & & & & \\
\hline 5.293 & 0.0385 & & & & \\
\hline $\begin{array}{l}4.825 \\
52\end{array}$ & & & & & \\
\hline $\begin{array}{r}5.2 \\
5.98\end{array}$ & & & & & \\
\hline 5.68 & 0.0325 & & 0.00175 & 14476 & 328 \\
\hline $\begin{array}{r}6.81 \\
10.12\end{array}$ & $\begin{array}{l}0.055 \\
0.095\end{array}$ & & $\begin{array}{l}0.00225 \\
0.00205\end{array}$ & $\begin{array}{l}53834 \\
82911\end{array}$ & $\begin{array}{l}2087 \\
2442\end{array}$ \\
\hline 0.111 & & & 0.000165 & 0.094101 & 103 \\
\hline 0.0759 & 0.004 & 33 & 0.000325 & -0.020201 & \\
\hline 0.08 & 0.00 & 9 & 0.000 & 0.21634 & \\
\hline 0.09 & & 14 & 17 & 765 & \\
\hline 0.17 & & 01089 & 0.000185 & 0.08432 & \\
\hline 0.11 & 0.017 & 0.0 & 227 & 0.011608 & \\
\hline 0.18 & & 4 & 14 & & \\
\hline 0.06 & & & 0.000 & & \\
\hline 0.25 & & & & & \\
\hline 0.2 & & & & & \\
\hline 0.075 & & & & & \\
\hline 0.1 & & & & & \\
\hline & & & & & \\
\hline & & & & & \\
\hline & & & & & \\
\hline & & & & & \\
\hline & & & & & \\
\hline & & & 117 & & \\
\hline 0.095 & 0.0065 & 0.00958 & 0.00012 & 0.04236 & 85.5 \\
\hline
\end{tabular}
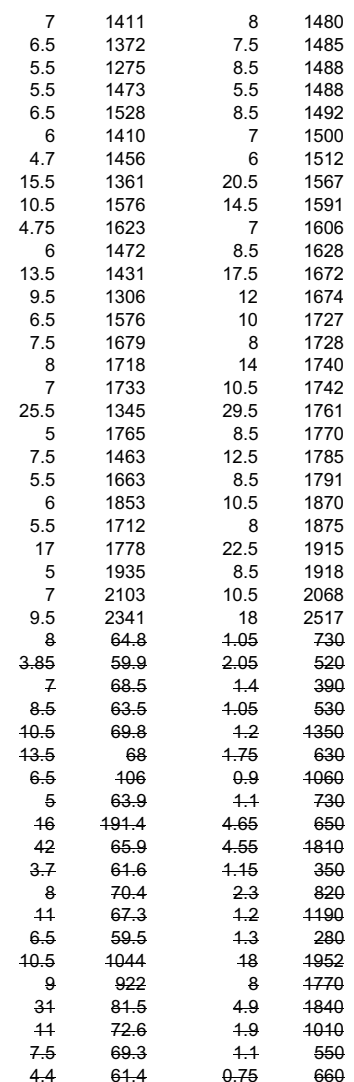

\begin{tabular}{|c|c|c|}
\hline 18.5 & 1480.0 & 185 \\
\hline 13.5 & 1485.0 & $\begin{array}{l}10.5 \\
13.5\end{array}$ \\
\hline 8.5 & 1488.0 & 8.5 \\
\hline 12.5 & 1488.0 & 12.5 \\
\hline & 1492.0 & 13.0 \\
\hline & 1500.0 & 11.0 \\
\hline 10.5 & 1512.0 & 10.5 \\
\hline 14.5 & 1567.0 & 14.5 \\
\hline $\begin{array}{r}15 \\
115\end{array}$ & 1591.0 & 15.0 \\
\hline 11.5 & 1606.0 & \\
\hline 12 & 1628.0 & 12.0 \\
\hline $\begin{array}{l}14 \\
20\end{array}$ & $\begin{array}{l}1672.0 \\
1674 .\end{array}$ & $\begin{array}{l}14.0 \\
200\end{array}$ \\
\hline 9 & 17270 & 9.0 \\
\hline 18 & 17280 & 180 \\
\hline 13 & 1740.0 & 130 \\
\hline 15.5 & 1742.0 & 15.5 \\
\hline 35.5 & 1761.0 & 35.5 \\
\hline & 1770.0 & 9.0 \\
\hline 1.5 & 1785.0 & 11.5 \\
\hline 11.5 & 1791.0 & 11.5 \\
\hline 10.5 & 1870.0 & 10.5 \\
\hline 11 & 1875.0 & 11.0 \\
\hline 19 & 1915.0 & 19.0 \\
\hline 10.5 & 1918.0 & 10.5 \\
\hline 12 & 2068.0 & 12.0 \\
\hline 8.5 & 2517.0 & 8.5 \\
\hline 115 & DISCARD & \#VALUE \\
\hline 110 & DISCARD & \#VALUE \\
\hline 155 & DISCARD & \#VALUE \\
\hline 135 & DISCARD & \#VALUE \\
\hline 105 & DISCARD & \#VALUE \\
\hline 795 & DISCARD & \#VALUE \\
\hline 75 & DISCARD & \#VALUE \\
\hline 110 & DISCARD & \#VALUE \\
\hline 205 & DISCARD & \#VALUE \\
\hline $\begin{aligned} 435 \\
95 \mathrm{t}\end{aligned}$ & $\begin{array}{l}\text { DISGARD } \\
\text { DISCARD }\end{array}$ & $\begin{array}{l}\text { \#VALUE } \\
\text { HVALUE }\end{array}$ \\
\hline 150 & DISCARD & \#VALUE \\
\hline 125 & DISCARD & \#VALUE \\
\hline $150 \mathrm{t}$ & DISCARD & \#VALUE \\
\hline $15.5 \mathrm{t}$ & DISCARD & \#VALUE \\
\hline $23 \mathrm{t}$ & DISGARD & \#VALUE \\
\hline $250 \mathrm{t}$ & DISCARD & \#VALUE: \\
\hline 150 & DISGARD & \#VALUE \\
\hline 110 & DISCARD & \#VALUE \\
\hline & & \#VALUE \\
\hline
\end{tabular}

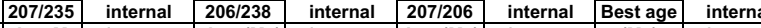

\begin{tabular}{|c|c|c|c|c|c|c|c|c|c|}
\hline 658 & 0.67 & 0.0507 & 0.00125 & 0.0866 & 0.0018 & 0.01251 & $\frac{10 \text { error }}{0.00024}$ & 0.28376 & . \\
\hline 271 & 0.94 & 0.0492 & 0.001 & 0.0991 & 0.00215 & 0.01458 & 0.000185 & 0.22922 & \\
\hline & 0.67 & 0.0485 & 0.00085 & 0.1029 & 0.0019 & 0.0153 & 0.0002 & 0.40337 & \\
\hline 136.8 & 0.77 & 0.0484 & 0.00125 & 0.1012 & 0.0025 & 0.01535 & 0.00016 & .14595 & \\
\hline 325 & 0.78 & 0.0505 & 0.00085 & 0.1091 & 0.00185 & 0.0156 & 0.000175 & .34825 & \\
\hline 161 & 0.98 & 0.056 & 0.0018 & 0.1265 & 0.00445 & 0.01632 & 0.000365 & .36096 & \\
\hline 387 & 0.62 & 0.0531 & 0.00095 & 0.1208 & 0.0024 & 0.01651 & 0.00029 & 0.46431 & \\
\hline 1405 & 0.62 & 0.0503 & 0.00055 & 0.1164 & 0.00155 & 0.01664 & 0.000165 & 0.51204 & \\
\hline 144.6 & 1.00 & 0.0487 & 0.0012 & 0.1125 & 0.0028 & 0.01666 & 0.000155 & 0.23685 & \\
\hline 556 & 0.81 & 0.0545 & 0.0009 & 0.1746 & 0.0031 & 0.02334 & 0.000305 & 0.45825 & \\
\hline 348 & 0.90 & 0.0511 & 0.00065 & 0.1732 & 0.00215 & 0.02463 & 0.00018 & 0.26939 & \\
\hline 231.9 & 1.12 & 0.0512 & 0.0007 & 0.1914 & 0.0026 & 0.02702 & 0.00021 & 0.31797 & \\
\hline 330 & 0.61 & 0.0531 & 0.0008 & 0.201 & 0.00315 & 0.02742 & 0.000235 & 0.31361 & \\
\hline 352 & 0.81 & 0.0494 & 0.0006 & 0.1868 & 0.00 & & 0.00019 & 0.30065 & \\
\hline 329.1 & 1.12 & 0.0499 & 0.0007 & 0.188 & 0.00275 & & & 0.35181 & \\
\hline 320.7 & 0.86 & 0.0498 & 0.00065 & 0.1922 & 0.00 & & & & \\
\hline 220.8 & 0.77 & & & & & & 0.000335 & 0.43436 & \\
\hline 287.9 & 1.11 & & 0.000 & & & & & 36987 & \\
\hline 776 & 0.27 & 0.05347 & & & & & & & \\
\hline 645 & 0.73 & 0.0547 & 0.000 & 0.4157 & & & 0.00047 & 0.48468 & \\
\hline 597 & 0.05 & 0.0547 & 0.000 & 0.429 & 0.0055 & 0.0569 & 0.0006 & 0.5993 & \\
\hline 359.4 & 1.33 & 0.0546 & 0.0005 & 0.4467 & 0.00425 & 4 & 0.0005 & 0.45403 & \\
\hline $\begin{array}{r}83.4 \\
111.3\end{array}$ & 1.97 & 0.0569 & & 0.509 & 0.0085 & 0.0663 & 0.001 & 0.46987 & \\
\hline 110.3 & 0.43 & 0.0556 & $\begin{array}{r}0.0007 \\
0.0055\end{array}$ & 0.505 & & 0.0667 & $\begin{array}{r}0.0007 \\
0.0005\end{array}$ & 0.38977 & \\
\hline $\begin{array}{l}275 \\
195\end{array}$ & 0.72 & $\begin{array}{l}0.0553 \\
0.0538\end{array}$ & $\begin{array}{l}0.00055 \\
0.00095\end{array}$ & 0.512 & 0.006 & 0.0671 & 0.00065 & 0.54965 & \\
\hline 195 & 0.02 & 0.0538 & 0.00095 & 0.506 & 0.009 & 0.0683 & 0.0011 & 0.38835 & \\
\hline
\end{tabular}

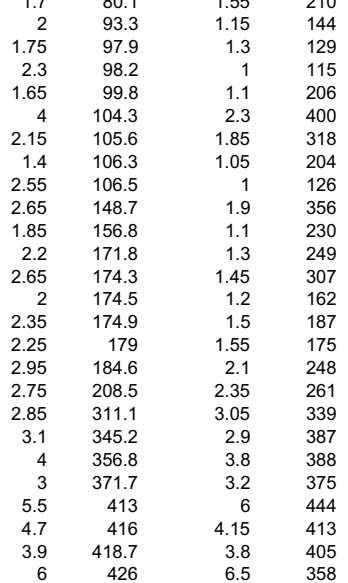

$\begin{array}{rr}49.5 & 80.1 \\ 38.5 & 93.3 \\ 34.5 & 97.9 \\ 49 & 98.2 \\ 34 & 99.8 \\ 65 & 104.3 \\ 37.5 & 105.6 \\ 23 & 106.3 \\ 47.5 & 106.5 \\ 34.5 & 148.7 \\ 26.5 & 156.8 \\ 28.5 & 171.8 \\ 32.5 & 174.3 \\ 25 & 174.5 \\ 29 & 174.9 \\ 27 & 179.0 \\ 34.5 & 184.6 \\ 29.5 & 208.5 \\ 19.5 & 311.1 \\ 20.5 & 345.2 \\ 23.5 & 356.8 \\ 21.5 & 371.7 \\ 37.5 & 413.0 \\ 28.5 & 416.0 \\ 21.5 & 418.7 \\ 3 & 42.0\end{array}$

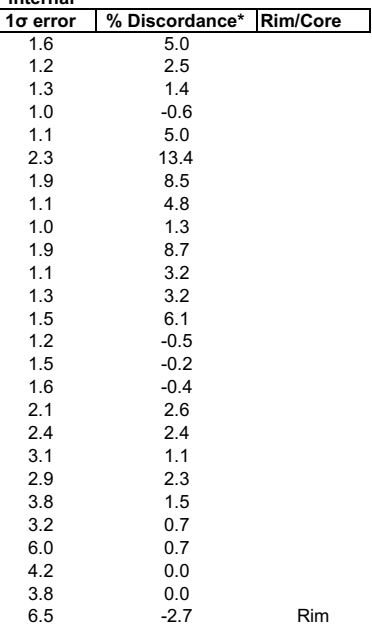




\begin{tabular}{|c|c|c|c|c|c|c|c|c|c|c|c|c|c|c|c|c|c|c|c|}
\hline Kiowa_848_64.FIN2 & 474 & 0.10 & 0.05932 & 0.000435 & 0.5603 & 0.00495 & 0.0686 & 0.0006 & 0.67336 & 450.9 & 3.2 & 427.8 & 3.7 & 571 & 15.5 & 427.8 & 3.7 & 5.1 & \\
\hline Kiowa_848_70.FIN2 & 339.8 & 0.79 & 0.05523 & 0.00036 & 0.5317 & 0.0034 & 0.06986 & 0.000325 & 0.3662 & 433.2 & 2.3 & 435.2 & 1.95 & 412 & 14.5 & 435.2 & 2.0 & -0.5 & \\
\hline Kiowa_848_14.FIN2 & 294 & 0.30 & 0.05431 & 0.00047 & 0.5422 & 0.0049 & 0.0722 & 0.00055 & 0.52785 & 439.1 & 3.25 & 450.1 & 3.4 & 369 & 19 & 450.1 & 3.4 & -2.5 & \\
\hline Kiowa_848_1.FIN2r & 670 & 0.15 & 0.0585 & 0.00195 & 0.631 & 0.0225 & 0.0785 & 0.0022 & 0.50216 & 495 & 13.5 & 487 & 13 & 520 & 70 & 487.0 & 13.0 & 1.6 & Rim \\
\hline Kiowa_848_89.FIN2 & 324 & 0.45 & 0.0591 & 0.00055 & 0.711 & 0.008 & 0.0871 & 0.0008 & 0.64902 & 543.9 & 4.8 & 537.9 & 4.75 & 556 & 20 & 537.9 & 4.8 & 1.1 & \\
\hline Kiowa_848_86.FIN2 & 82.4 & 0.59 & 0.0691 & 0.0014 & 0.878 & 0.018 & 0.0941 & 0.00145 & 0.36097 & 633 & 9.5 & 579 & 8.5 & 820 & 41.5 & 579.0 & 8.5 & 8.5 & \\
\hline Kiowa_848_91.FIN2 & 329 & 0.63 & 0.06229 & 0.00038 & 0.853 & 0.0085 & 0.0992 & 0.001 & 0.8027 & 624.8 & 4.7 & 609 & 6 & 679 & 13.5 & 609.0 & 6.0 & 2.5 & \\
\hline Kiowa_848_69.FIN2 & 89.3 & 1.26 & 0.0673 & 0.001 & 0.972 & 0.015 & 0.1046 & 0.0014 & 0.41753 & 685 & 7.5 & 641 & 8 & 817 & 32 & 641.0 & 8.0 & 6.4 & \\
\hline Kiowa_848_94.FIN2 & 227 & 0.24 & 0.0598 & 0.0005 & 0.873 & 0.0075 & 0.1057 & 0.0007 & 0.43607 & 635.9 & 4.15 & 647.4 & 4.15 & 586 & 18 & 647.4 & 4.2 & -1.8 & \\
\hline Kiowa_848_32.FIN2 & 63.8 & 0.71 & 0.0619 & 0.0007 & 0.905 & 0.01 & 0.1067 & 0.0006 & 0.30541 & 652 & 5.5 & 653.3 & 3.65 & 643 & 23.5 & 653.3 & 3.7 & -0.2 & \\
\hline Kiowa_848_60.FIN2 & 50.2 & 1.58 & 0.0689 & 0.0009 & 1.637 & 0.0245 & 0.1723 & 0.00235 & 0.59219 & 986 & 10 & 1024 & 13 & 893 & 25 & 893.0 & 25.0 & -14.7 & \\
\hline Kiowa_848_49.FIN2 & 57 & 0.57 & 0.0724 & 0.00065 & 1.837 & 0.017 & 0.1842 & 0.0012 & 0.38255 & 1058 & 6 & 1089 & 6.5 & 986 & 17.5 & 986.0 & 17.5 & -10.4 & \\
\hline Kiowa_848_30.FIN2 & 94.5 & 0.85 & 0.0732 & 0.00055 & 1.787 & 0.0125 & 0.1774 & 0.0009 & 0.30225 & 1039.1 & 4.55 & 1052 & 5 & 1009 & 15.5 & 1009.0 & 15.5 & -4.3 & \\
\hline Kiowa_848_47.FIN2 & 141.1 & 0.98 & 0.0739 & 0.00055 & 1.833 & 0.015 & 0.18 & 0.00115 & 0.45195 & 1055 & 5.5 & 1068 & 6.5 & 1029 & 16 & 1029.0 & 16.0 & -3.8 & \\
\hline Kiowa_848_68.FIN2 & 336 & 1.29 & 0.07373 & 0.000365 & 1.918 & 0.0125 & 0.1879 & 0.0012 & 0.65597 & 1087.3 & 4.25 & 1110 & 6.5 & 1032 & 10.5 & 1032.0 & 10.5 & -7.6 & \\
\hline Kiowa_848_79.FIN2 & 130.7 & 0.29 & 0.075 & 0.00105 & 1.854 & 0.03 & 0.1788 & 0.00285 & 0.61072 & 1057 & 10.5 & 1058 & 15.5 & 1042 & 29.5 & 1042.0 & 29.5 & -1.5 & \\
\hline Kiowa_848_28.FIN2 & 104 & 0.40 & 0.0755 & 0.00085 & 2.093 & 0.029 & 0.2014 & 0.00245 & 0.63255 & 1140 & 9.5 & 1184 & 13.5 & 1062 & 22 & 1062.0 & 22.0 & -11.5 & \\
\hline Kiowa_848_16.FIN2c & 37 & 0.39 & 0.076 & 0.0011 & 1.876 & 0.0365 & 0.1787 & 0.00235 & 0.52795 & 1069 & 13 & 1059 & 13 & 1083 & 28.5 & 1083.0 & 28.5 & 2.2 & Core \\
\hline Kiowa_848_95.FIN2 & 282 & 0.31 & 0.0771 & 0.0006 & 1.718 & 0.0165 & 0.1605 & 0.00135 & 0.65905 & 1012 & 6 & 959 & 7.5 & 1114 & 14.5 & 1114.0 & 14.5 & 13.9 & \\
\hline Kiowa_848_15.FIN2 & 5.37 & 0.62 & 0.082 & 0.0023 & 2.24 & 0.07 & 0.2054 & 0.0045 & 0.51244 & 1176 & 22.5 & 1198 & 24.5 & 1120 & 55 & 1120.0 & 55.0 & -7.0 & \\
\hline $\begin{array}{l}\text { Kiowa_848_48.FIN2 } \\
\text { KKiowa } 848 \text { 45FIN2 }\end{array}$ & $\begin{array}{r}202.6 \\
51.7\end{array}$ & 0.49 & 0.0779 & 0.00115 & 1.999 & 0.0305 & 0.1879 & 0.00285 & 0.50619 & 1114 & 10.5 & 1108 & 15.5 & 1128 & 30 & 1128.0 & 30.0 & 1.8 & \\
\hline $\begin{array}{l}\text { Kiowa_848_45.FIN2 } \\
\text { Kiowa_848_83.FIN2 }\end{array}$ & $\begin{array}{r}51.7 \\
137.4\end{array}$ & $\begin{array}{l}0.77 \\
0.60\end{array}$ & $\begin{array}{r}0.078 \\
0.0783\end{array}$ & $\begin{array}{r}0.0009 \\
0.00055\end{array}$ & $\begin{array}{l}2.013 \\
2.125\end{array}$ & $\begin{array}{r}0.03 \\
0.0185\end{array}$ & $\begin{array}{l}0.1869 \\
0.1962\end{array}$ & $\begin{array}{r}0.0021 \\
0.00145\end{array}$ & 0.64449 & $\begin{array}{l}1114 \\
1155\end{array}$ & 10.5 & $\begin{array}{l}1103 \\
1155\end{array}$ & $\begin{array}{r}11.5 \\
8\end{array}$ & $\begin{array}{l}1131 \\
1148\end{array}$ & 23.5 & 1131.0 & 23.5 & 2.5 & \\
\hline 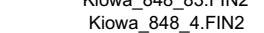 & $\begin{array}{r}151.4 \\
291\end{array}$ & 0.34 & $\begin{array}{l}0.0783 \\
0.0809\end{array}$ & $\begin{array}{r}0.00055 \\
0.0012\end{array}$ & $\begin{array}{l}2.125 \\
1.949\end{array}$ & $\begin{array}{l}0.0185 \\
0.034\end{array}$ & 0.1962 & $\begin{array}{l}0.00145 \\
0.00305\end{array}$ & 0.60462 & $\begin{array}{l}1155 \\
1089\end{array}$ & $\begin{array}{r}6 \\
12\end{array}$ & $\begin{array}{l}1155 \\
1041\end{array}$ & $\begin{array}{r}8 \\
17\end{array}$ & $\begin{array}{l}1148 \\
1185\end{array}$ & 15 & 1148.0 & 15.0 & -0.6 & \\
\hline Kiowa 848 - 1.FIN2c & 293 & 0.38 & 0.0822 & 0.00055 & $\begin{array}{r}. .349 \\
2.391\end{array}$ & 0.021 & $\begin{array}{l}0.158 \\
0.2111\end{array}$ & $\begin{array}{l}0.00305 \\
0.00195\end{array}$ & $\begin{array}{l}0.65936 \\
0.73855\end{array}$ & $\begin{array}{l}1089 \\
1238\end{array}$ & $\begin{array}{l}12 \\
6.5\end{array}$ & $\begin{array}{l}1041 \\
1234\end{array}$ & $\begin{array}{r}17 \\
105\end{array}$ & $\begin{array}{l}1185 \\
1245\end{array}$ & $\begin{array}{l}29.5 \\
13.5\end{array}$ & $\begin{array}{l}185.0 \\
1845 .\end{array}$ & $\begin{array}{l}29.5 \\
135\end{array}$ & $\begin{array}{l}12.2 \\
0.9\end{array}$ & Core \\
\hline Kiowa_848_8.FIN2 & 115.2 & 0.43 & 0.0833 & 0.00075 & 2.382 & 0.025 & 0.2073 & 0.00205 & 0.57988 & 1235 & 7.5 & 1214 & 11 & 1270 & 18 & 1270.0 & 18.0 & 4.4 & onto \\
\hline Kiowa_848_77.FIN2 & 176.9 & 0.32 & 0.0847 & 0.00065 & 2.501 & 0.0265 & 0.2133 & 0.0017 & 0.71439 & 1270 & 7.5 & 1246 & 9 & 1304 & 14 & 1304.0 & 14.0 & 4.4 & \\
\hline Kiowa_848_11.FIN2 & 49 & 0.61 & 0.0873 & 0.002 & 2.16 & 0.055 & 0.1766 & 0.00265 & 0.23441 & 1158 & 17 & 1046 & 14.5 & 1361 & 46 & 1361.0 & 46.0 & 23.1 & \\
\hline Kiowa_848_71.FIN2 & 33.6 & 0.10 & 0.0886 & 0.0009 & 3.031 & 0.0335 & 0.2482 & 0.00165 & 0.44819 & 1412 & 8 & 1429 & 8.5 & 1377 & 19.5 & 1377.0 & 19.5 & -3.8 & \\
\hline Kiowa_848_5.FIN2 & 53.2 & 1.07 & 0.0904 & 0.00105 & 2.88 & 0.031 & 0.235 & 0.0031 & 0.52653 & 1374 & 8.5 & 1362 & 16 & 1409 & 22.5 & 1409.0 & 22.5 & 3.3 & \\
\hline Kiowa_848_87.FIN2 & 275 & 0.25 & 0.0922 & 0.00075 & 3.401 & 0.0335 & 0.2663 & 0.0023 & 0.61958 & 1500 & 8 & 1520 & 12 & 1462 & 16 & 1462.0 & 16.0 & -4.0 & \\
\hline Kiowa_848_9.FIN2 & 93.5 & 0.76 & 0.0927 & 0.00065 & 3.066 & 0.0265 & 0.2401 & 0.00185 & 0.62987 & 1423 & 6.5 & 1386 & 9.5 & 1473 & 13.5 & 1473.0 & 13.5 & 5.9 & \\
\hline Kiowa_848_85.FIN2 & 139.5 & 0.51 & 0.0942 & 0.0006 & 3.427 & 0.024 & 0.2636 & 0.0019 & 0.64921 & 1508 & 5.5 & 1510 & 10 & 1505 & 12 & 1505.0 & 12.0 & -0.3 & \\
\hline Kiowa_848_52.FIN2 & 256.1 & 0.49 & 0.0947 & 0.00065 & 3.637 & 0.027 & 0.2784 & 0.0019 & 0.57539 & 1555 & 6 & 1582 & 9.5 & 1513 & 13 & 1513.0 & 13.0 & -4.6 & \\
\hline Kiowa_848_53.FIN2 & 132.7 & 0.70 & 0.0967 & 0.0016 & 3.38 & 0.065 & 0.2561 & 0.0045 & 0.58238 & 1487 & 15 & 1464 & 22.5 & 1520 & 32 & 1520.0 & 32.0 & 3.7 & \\
\hline Kiowa_848_46.FIN2 & 107.2 & 0.38 & 0.0961 & 0.0007 & 3.547 & 0.035 & 0.267 & 0.0022 & 0.69912 & 1536 & 8 & 1525 & 11 & 1545 & 13.5 & 1545.0 & 13.5 & 1.3 & \\
\hline Kiowa_848_13.FIN2 & 273 & 0.21 & 0.0974 & 0.00095 & 3.62 & 0.055 & 0.2679 & 0.00315 & 0.7777 & 1545 & 12.5 & 1527 & 16 & 1564 & 17 & 1564.0 & 17.0 & 2.4 & \\
\hline Kiowā_848_2.FIN2 & 162 & 0.64 & 0.0976 & 0.00055 & 4.09 & 0.0365 & 0.305 & 0.00275 & 0.76379 & 1648 & 7.5 & 1714 & 13.5 & 1573 & 11 & 1573.0 & 11.0 & -9.0 & \\
\hline Kiowa_848_27.FIN2 & 191 & 0.75 & 0.1001 & 0.0008 & 2.976 & 0.038 & 0.2154 & 0.0023 & 0.77284 & 1396 & 10 & 1256 & 12.5 & 1617 & 15.5 & 1617.0 & 15.5 & 22.3 & \\
\hline Kiowa_848_54.FIN2 & 249.3 & 0.66 & 0.1028 & 0.0009 & 3.943 & 0.0435 & 0.2791 & 0.0034 & 0.76297 & 1616 & 9.5 & 1583 & 17.5 & 1665 & 16.5 & 1665.0 & 16.5 & 4.9 & \\
\hline Kiowa_848_34.FIN2 & 88.3 & 0.60 & 0.1041 & 0.00125 & 4.16 & 0.065 & 0.2921 & 0.0047 & 0.68605 & 1664 & 12.5 & 1652 & 23 & 1678 & 23 & 1678.0 & 23.0 & 1.5 & \\
\hline Kiowa_848_40.FIN2 & 161.6 & 0.57 & 0.10335 & 0.000485 & 4.388 & 0.0275 & 0.3065 & 0.0018 & 0.66624 & 1708 & 5 & 1722 & 9 & 1686 & 9 & 1686.0 & 9.0 & -2.1 & \\
\hline Kiowa_848_20.FIN2 & 54.6 & 0.47 & 0.104 & 0.00065 & 4.38 & 0.031 & 0.3062 & 0.002 & 0.61056 & 1708 & 6 & 1724 & 10 & 1692 & 11.5 & 1692.0 & 11.5 & -1.9 & \\
\hline Kiowa_848_78.FIN2 & 255 & 0.42 & 0.1039 & 0.00075 & 4.588 & 0.044 & 0.32 & 0.00325 & 0.72018 & 1745 & 8 & 1791 & 15.5 & 1703 & 14 & 1703.0 & 14.0 & -5.2 & \\
\hline Kiowa_848_90.FIN2 & 585 & 0.08 & 0.105 & 0.0007 & 4.491 & 0.0365 & 0.3104 & 0.0026 & 0.65526 & 1730 & 7 & 1741 & 13 & 1707 & 12 & 1707.0 & 12.0 & -2.0 & \\
\hline $\begin{array}{l}\text { Kiowa_848_56.FIN2 } \\
\text { Kina }\end{array}$ & 41.7 & 1.70 & 0.1055 & 0.00095 & 4.69 & 0.055 & 0.3223 & 0.0027 & 0.52365 & 1763 & 8.5 & 1799 & 13 & 1710 & 16 & 1710.0 & 16.0 & -5.2 & \\
\hline $\begin{array}{l}\text { Kiowa_848_75.FIN2 } \\
\text { Kiowa } 848 \text { 67.FIN2 }\end{array}$ & $\begin{array}{l}76.5 \\
426\end{array}$ & $\begin{array}{l}2.08 \\
0.36\end{array}$ & $\begin{array}{r}0.1068 \\
0.10667\end{array}$ & 0.00135 & $\begin{array}{r}4.68 \\
4.72\end{array}$ & 0.06 & 0.3215 & $\begin{array}{r}0.0047 \\
0.0225\end{array}$ & 0.63606 & 1763 & 11 & 1797 & 23.5 & 1720 & 23 & 1720.0 & 23.0 & -4.5 & \\
\hline $\begin{array}{l}\text { Kiowa_848_67.FIN2 } \\
\text { Kiowa_848_29.FIN2 }\end{array}$ & $\begin{array}{l}426 \\
125\end{array}$ & $\begin{array}{l}0.36 \\
0.21\end{array}$ & $\begin{array}{r}0.10667 \\
0.1071\end{array}$ & $\begin{array}{l}0.00046 \\
0.0006\end{array}$ & $\begin{array}{l}4.172 \\
4619\end{array}$ & $\begin{array}{l}0.0355 \\
0.0415\end{array}$ & 0.2827 & $\begin{array}{l}0.00225 \\
0.00295\end{array}$ & $\begin{array}{l}0.85384 \\
081332\end{array}$ & $\begin{array}{l}1665 \\
1750\end{array}$ & $\begin{array}{r}7 \\
75\end{array}$ & $\begin{array}{l}1604 \\
1754\end{array}$ & $\begin{array}{l}11.5 \\
14.5\end{array}$ & $\begin{array}{l}1742 \\
1748\end{array}$ & 7.5 & 1742.0 & $\begin{array}{r}7.5 \\
100\end{array}$ & 7.9 & \\
\hline 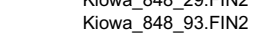 & $\begin{array}{l}290 \\
294-10\end{array}$ & 0.41 & 0.1081 & 0.0007 & $\begin{array}{l}4.019 \\
5.3\end{array}$ & 0.0405 & $\begin{array}{l}0.3732 \\
0.3549\end{array}$ & $\begin{array}{r}0.00295 \\
0.0031\end{array}$ & 0.81332 & $\begin{array}{l}1750 \\
1866\end{array}$ & $\begin{array}{r}7.5 \\
6.5\end{array}$ & $\begin{array}{l}1754 \\
1956\end{array}$ & $\begin{array}{r}14.5 \\
14.5\end{array}$ & 1748 & 10 & 1748.0 & 10.0 & -0.3 & \\
\hline Kiowa_848_44.FIN2 & 404 & 0.76 & 0.1098 & 0.00075 & 5.5 & 0.04 & 0.3639 & 0.00275 & 0.58681 & 1898 & $\begin{array}{l}6.5 \\
6.5\end{array}$ & $\begin{array}{l}1956 \\
1999\end{array}$ & $\begin{array}{r}14.5 \\
13\end{array}$ & $\begin{array}{l}\begin{array}{l}1761 \\
1790\end{array}\end{array}$ & $\begin{array}{l}12 \\
12\end{array}$ & $\begin{array}{l}1761.0 \\
1790.0\end{array}$ & $\begin{array}{l}12.0 \\
12.0\end{array}$ & $\begin{array}{l}-11.1 \\
-11.7\end{array}$ & \\
\hline Kiowa_848_18.FIN2 & 547 & 0.33 & 0.1113 & 0.00065 & 4.998 & 0.032 & 0.3259 & 0.00235 & 0.68126 & 1817 & 5.5 & 1817 & 11.5 & 1815 & 10 & 1815.0 & 10.0 & -0.1 & \\
\hline Kiowa_848_31.FIN2 & 386 & 0.39 & 0.11162 & 0.000465 & 5.055 & 0.031 & 0.329 & 0.00215 & 0.819 & 1827 & 5.5 & 1832 & 10.5 & 1825 & 8 & 1825.0 & 8.0 & -0.4 & \\
\hline Kiowa_848_62.FIN2 & 174.2 & 0.25 & 0.1476 & 0.0015 & 8.11 & 0.115 & 0.3985 & 0.00475 & 0.71632 & 2237 & 13 & 2156 & 22 & 2306 & 17.5 & 2306.0 & 17.5 & 6.5 & \\
\hline Kiowa_848_96.FIN2 & 57.7 & 0.64 & 0.1569 & 0.00185 & 8.39 & 0.135 & 0.39 & 0.0065 & 0.76173 & 2277 & 13 & 2112 & 29.5 & 2411 & 20.5 & 2411.0 & 20.5 & 12.4 & \\
\hline Kiowa_848_88.FIN2 & 106 & 0.54 & 0.1652 & 0.00225 & 10.22 & 0.17 & 0.449 & 0.007 & 0.61228 & 2442 & 15.5 & 2388 & 32.5 & 2501 & 22.5 & 2501.0 & 22.5 & 4.5 & \\
\hline Kiowa_848_92.FIN2 & 82.1 & 1.11 & 0.184 & 0.0013 & 14.5 & 0.135 & 0.567 & 0.006 & 0.75497 & 2780 & 9 & 2901 & 24.5 & 2681 & 12 & 2681.0 & 12.0 & -8.2 & \\
\hline Kiowa_848_50.FIN2 & 220 & 0.15 & 0.1888 & 0.0008 & 13.66 & 0.075 & 0.5251 & 0.0033 & 0.74933 & 2725 & 5 & 2719 & 14 & 2730 & 7 & 2730.0 & 7.0 & 0.4 & \\
\hline Kiowa_848_55.FIN2 & 315 & 0.23 & 0.1939 & 0.0017 & 15.11 & 0.16 & 0.57 & 0.0075 & 0.72829 & 2817 & 10 & 2904 & 29.5 & 2765 & 14 & 2765.0 & 14.0 & -5.0 & \\
\hline Kiowa_848_17.FIN2 & 221 & 0.48 & 0.15 & 0.005 & 0.386 & 0.016 & 0.01807 & 0.000225 & 0.68708 & 322 & 11.5 & 115.4 & 1.4 & 2190 & & - DISCARD & \#VALUE! & 64.2 & \\
\hline Kiowa_848_21.FIN2 & 188.6 & 0.85 & 0.204 & 0.0075 & 0.487 & 0.021 & 0.01638 & 0.00025 & 0.76754 & 394 & 15 & 104.7 & 1.6 & 2600 & & DISCARD & \#VALUE! & 73.4 & \\
\hline Kiowa_848_63.FIN2 & 65.1 & 2.21 & 0.0821 & 0.00195 & 0.414 & 0.0105 & 0.03646 & 0.000405 & 0.35808 & 349 & 7.5 & 230.8 & 2.55 & 1208 & & DISCARD & \#VALUE! & 33.9 & \\
\hline Kiowa_848_72.FIN2 & 53.6 & 5.84 & 0.0891 & 0.00105 & 1.648 & 0.0225 & 0.1343 & 0.0013 & 0.54015 & 986 & 8.5 & 811 & 7.5 & 1401 & & DISCARD & \#VALUE! & 17.7 & \\
\hline ample Name: Kiowa-973 & & & & internal & & internal & & internal & & $207 / 235$ & internal & 206/238 & internal & \begin{tabular}{|l|}
$207 / 206$ \\
\end{tabular} & internal & Best age & internal & & \\
\hline Analysis ID & {$[\mathrm{U}] \mathrm{ppm}$} & $\mathrm{Th} / \mathrm{U}$ & 207/206 & $1 \sigma$ error & 207/235 & $1 \sigma$ error & 206/238 & $1 \sigma$ error & RHO & Age Ma & $1 \sigma$ error & Age (Ma) & 10 error & $\mid$ Age (Ma) & 10 error & (Ma) & $1 \sigma$ error & \% Discordance ${ }^{*}$ & Rim/Core \\
\hline Kiowa_973_89.FIN2 & 96.7 & 0.54 & 0.0519 & 0.00175 & 0.0733 & 0.00235 & 0.01042 & 0.000195 & 0.12543 & 71.5 & 2.25 & 66.8 & 1.25 & 240 & 65 & 66.8 & 1.3 & 6.6 & \\
\hline Kiowa_973_63.FIN2 & 153.5 & 0.4 & 0.0501 & 0.00125 & 0.0 & 0.00175 & 0.01046 & 0.00011 & 0.11673 & & 1.7 & 67.1 & 0.7 & 185 & 49 & 67. & 0.7 & 4.8 & \\
\hline Kiowa_973_41.FIN2 & 137.9 & 0.46 & 0.0484 & 0.00125 & 0.0715 & 0.0018 & 0.01067 & 0.000125 & 0.058437 & 69.9 & 1.7 & 68.4 & 0.8 & 108 & 47.5 & 68.4 & 0.8 & 2.1 & \\
\hline Kiowa_973_45.FIN2 & 57.9 & 1.01 & 0.0468 & 0.00215 & 0.0708 & 0.0031 & 0.0114 & 0.00016 & 0.06797 & 68.9 & 2.9 & 73.1 & 1.05 & 0 & 75 & 73.1 & 1.1 & -6.1 & \\
\hline Kiowa_973_67.FIN2 & 258.3 & 0.84 & 0.0498 & 0.001 & 0.0799 & 0.00145 & 0.01176 & 0.0001 & 0.14314 & 77.9 & 1.35 & 75.4 & 0.65 & 174 & 38.5 & 75.4 & 0.7 & 3.2 & \\
\hline Kiowa_973_30.FIN2 & 151.5 & 0.90 & 0.0505 & 0.0022 & 0.082 & 0.00345 & 0.01181 & 0.000215 & 0.10754 & 79.8 & 3.25 & 75.7 & 1.4 & 190 & 85 & 75.7 & 1.4 & 5.1 & \\
\hline Kiowa_973_57.FIN2 & 111.2 & 0.78 & 0.0464 & 0.0012 & 0.0774 & 0.00185 & 0.01217 & 0.00012 & 0.15572 & 75.5 & 1.75 & 78 & 0.75 & 27 & 45 & 78.0 & 0.8 & -3.3 & \\
\hline Kiowa_973_96.FIN2 & 223 & 0.74 & 0.0534 & 0.0013 & 0.0917 & 0.00205 & 0.01261 & 0.000105 & 0.016031 & 88.8 & 1.9 & 80.8 & 0.65 & 272 & 42 & 80.8 & 0.7 & 9.0 & \\
\hline $\begin{array}{l}\text { Kiowa_973_52.FIN2 } \\
\text { Kiowa 973 106 FIN2 }\end{array}$ & $\begin{array}{r}233.5 \\
1259\end{array}$ & $\begin{array}{l}0.61 \\
0.53\end{array}$ & $\begin{array}{r}0.0482 \\
0.04732\end{array}$ & $\begin{array}{r}0.00095 \\
0.000485\end{array}$ & $\begin{array}{l}0.0918 \\
0.0935\end{array}$ & $\begin{array}{r}0.00185 \\
0.001\end{array}$ & $\begin{array}{l}0.01378 \\
0.01436\end{array}$ & $\begin{array}{r}0.000145 \\
0.0001\end{array}$ & $\begin{array}{l}0.31386 \\
0.41954\end{array}$ & $\begin{array}{r}89 \\
907\end{array}$ & $\begin{array}{r}1.7 \\
0.95\end{array}$ & $\begin{array}{l}88.2 \\
91.9\end{array}$ & $\begin{array}{r}0.9 \\
0.65\end{array}$ & $\begin{array}{l}124 \\
74\end{array}$ & $\begin{array}{r}39 \\
205\end{array}$ & $\begin{array}{l}88.2 \\
91.9\end{array}$ & $\begin{array}{l}0.9 \\
0.7\end{array}$ & $\begin{array}{c}0.9 \\
-1.3\end{array}$ & \\
\hline & & & & & & & & & & & & & & & & & & & \\
\hline
\end{tabular}




\begin{tabular}{|c|c|c|c|c|c|c|c|c|c|c|}
\hline Kiowa 973 130.FIN2 & 415 & 138 & 0.0474 & 0.0006 & 0.1007 & 0.0012 & 1547 & & 0.115 & .4 \\
\hline Kiowa 973 132.FIN2 & 299 & 0.45 & 0.0482 & 0.0008 & 0.102 & 0.0016 & 0.01554 & 0.000175 & 0.29216 & $98.5>2>0$ \\
\hline Kiowa 973 76.FIN2 & 274 & 0.71 & 0.0497 & 0.00095 & 0.1045 & 0.00185 & 0.01556 & 00175 & 0.22108 & 1007 \\
\hline Kiowa 973 23 FIN2 & 482 & 0.64 & 0.0492 & 0.00075 & 0.1048 & 0.0016 & 0.01556 & 0.000135 & 0.36108 & \\
\hline $\begin{array}{l}\text { Kiowa } 973 \text { 4. FIN2 } \\
\text { Kallo }\end{array}$ & 273 & 1.26 & 0.0491 & 0.00095 & 0.1088 & 0.00195 & 0.01619 & 0.00015 & 0.086996 & 10 \\
\hline Kiowa 973 128.FIN2 & 630 & 0.31 & 0.0493 & 0.0007 & 0.1098 & 0.00155 & 0.01638 & 0.000205 & 0.46627 & 105.7 \\
\hline Kiowa 973 33.FIN2 & 734 & 0.39 & 0.0485 & 0.00055 & 0.1102 & 0.0013 & 0.01664 & 0.00018 & 0.56014 & 106.1 \\
\hline Kiowa 973 50.FIN2 & 231.3 & 0.60 & 0.0497 & 0.0009 & 0.1142 & 0.00205 & 0.01664 & 0.00013 & 0.11391 & 109.6 \\
\hline Kiowa_973_133.FIN2 & 249 & 1.45 & 0.0496 & 0.0009 & 0.1164 & 0.00225 & 0.01708 & 0.000215 & 0.47317 & 111.5 \\
\hline Kiowa_973_37.FIN2 & 211.2 & 1.91 & 0.0527 & 0.00105 & 0.158 & 0.0034 & 0.02178 & 0.00031 & 0.43413 & 148.6 \\
\hline Kiowa_973_56.FIN2 & 102.5 & 0.89 & 0.05 & 0.001 & 0.1722 & 0.00325 & 0.02509 & 0.000185 & 0.097779 & 160.7 \\
\hline Kiowa_973_58.FIN2 & 319.8 & 0.80 & 0.0509 & 0.0006 & 0.1822 & 0.0021 & 0.02592 & 0.0002 & 0.22596 & 169.7 \\
\hline Kiowa_973_60.FIN2 & 1096 & 0.45 & 0.0554 & 0.00065 & 0.1975 & 0.00295 & 0.02612 & 0.00039 & 0.69532 & 182.7 \\
\hline Kiowa_973_126.FIN2 & 300.9 & 0.69 & 0.0508 & 0.0006 & 0.1834 & 0.00225 & 0.02625 & 0.000195 & 0.32674 & 171.2 \\
\hline Kiowa_973_10.FIN2 & 101.7 & 0.49 & 0.0486 & 0.0009 & 0.1758 & 0.00305 & 0.02631 & 0.00024 & 0.077724 & 164.6 \\
\hline Kiowa_973_80.FIN2 & 1182 & 2.75 & 0.05198 & 0.00041 & 0.1891 & 0.0018 & 0.02632 & 0.000235 & 0.50616 & 175.6 \\
\hline Kiowa_973_32.FIN2r & 593 & 0.64 & 0.0506 & 0.00095 & 0.1871 & 0.00315 & 0.02691 & 0.00043 & 0.23028 & 174 \\
\hline Kiowa_973_137.FIN2 & 277 & 1.58 & 0.0522 & 0.0012 & 0.1935 & 0.00485 & 0.0272 & 0.0006 & 0.49155 & 179.2 \\
\hline Kiowa_973_99.FIN2 & 85.4 & 2.28 & 0.052 & 0.0011 & 0.1975 & 0.00415 & 0.02782 & 0.000365 & 0.25585 & 182.1 \\
\hline Kiowa_973_54.FIN2 & 435 & 1.20 & 0.0501 & 0.00065 & 0.1934 & 0.00215 & 0.02819 & 0.000225 & 0.20607 & 179.7 \\
\hline Kiowa_973_77.FIN2 & 474 & 0.23 & 0.0498 & 0.0005 & 0.1955 & 0.0022 & 0.02853 & 0.00018 & 0.39004 & 181 \\
\hline Kiowa_973_20.FIN2 & 564 & 1.11 & 0.0507 & 0.00055 & 0.2046 & 0.00235 & 0.02926 & 0.00025 & 0.51412 & 188.7 \\
\hline Kiowa_973_36.FIN2 & 300 & 1.39 & 0.0517 & 0.0006 & 0.2101 & 0.0024 & 0.02955 & 0.00025 & 0.34704 & 193.4 \\
\hline Kiowa_973_55.FIN2 & 120.8 & 0.79 & 0.0498 & 0.00085 & 0.2027 & 0.0034 & 0.02958 & 0.000235 & 0.22447 & 186.8 \\
\hline Kiowa_973_90.FIN2 & 825 & 0.56 & 0.05265 & 0.0004 & 0.2693 & 0.0026 & 0.03745 & 0.000335 & 0.69288 & 242.3 \\
\hline Kiowa_973_71.FIN2 & 210 & 0.74 & 0.0551 & 0.00055 & 0.505 & 0.0055 & 0.0663 & 0.0005 & 0.4807 & 414.2 2 . \\
\hline Kiowa_973_46.FIN2 & 238 & 0.27 & 0.0551 & 0.0005 & 0.5158 & 0.0046 & 0.068 & 0.00055 & 0.42606 & 422.3 \\
\hline Kiowa_973_84.FIN2 & 356 & 0.49 & 0.05553 & 0.000475 & 0.554 & 0.005 & 0.0724 & 0.0006 & 0.51245 & 446.6 \\
\hline Kiowa_973_121.FIN2 & 278 & 0.50 & 0.0596 & 0.00115 & 0.605 & 0.0105 & 0.0753 & 0.0013 & 0.39232 & 479 \\
\hline Kiowa_973_117.FIN2 & 421 & 0.35 & 0.056 & 0.0008 & & & 0.0765 & 0.0012 & 0.49821 & 466 \\
\hline Kiowa_973_42.FIN2 & 208.7 & 0.80 & 0.05673 & 0.000445 & 0.6003 & 0.00465 & 0.07687 & 0.00043 & 0.36786 & 476.8 \\
\hline Kiowa_973_111.FIN2 & 191 & 0.89 & 0.0606 & 0.0007 & 0.669 & 0.0075 & 0.0809 & 0.00065 & 0.27541 & 518.5 \\
\hline Kiowa 973 38.FIN2 & 357 & 0.74 & 0.074 & 0.0009 & 0.984 & 0.013 & 0.0967 & 0.00135 & 0.53072 & 694 \\
\hline Kiowa_973_75.FIN2 & 210 & 0.91 & 0.06116 & 0.000475 & 0.839 & 0.0065 & 0.0997 & 0.0006 & 0.38289 & 618.6 \\
\hline Kiowa_973_34.FIN2 & 123 & 0.58 & 0.0609 & 0.00085 & 0.845 & 0.011 & 0.1013 & 0.00095 & 0.20637 & 619 \\
\hline Kiowa_973_116.FIN2 & 82.3 & 0.25 & 0.0608 & 0.00065 & 0.9 & 0.01 & 0.1078 & 0.00085 & 0.38777 & 649 \\
\hline Kiowa_973_115.FIN2 & 271 & 0.18 & 0.0628 & 0.0008 & 0.968 & 0.0125 & 0.1144 & 0.0018 & 0.61302 & 684 \\
\hline Kiowa_973_28.FIN2 & 61 & 0.41 & 0.0699 & 0.00075 & 1.522 & 0.015 & 0.158 & 0.001 & 0.27924 & 937 \\
\hline Kiowa_973_66.FIN2 & 366 & 0.06 & 0.0726 & 0.0005 & 1.764 & 0.0135 & 0.1769 & 0.0016 & 0.7023 & 1030 \\
\hline Kiowa_973_108.FIN2 & 36.5 & 1.81 & 0.0748 & 0.00115 & 1.616 & 0.023 & 0.1595 & 0.00185 & 0.35493 & 973 \\
\hline Kiowa_973_3.FIN2 & 70.4 & 0.56 & 0.0748 & 0.00065 & 1.87 & 0.016 & 0.1814 & 0.00115 & 0.32515 & 1068 \\
\hline Kiowa_973_135.FIN2 & 595 & 0.30 & 0.0746 & 0.0005 & 1.547 & 0.0185 & 0.1509 & 0.0019 & 0.8248 & 947 \\
\hline Kiowa_973_102.FIN2 & 139 & 0.36 & 0.0751 & 0.0006 & 1.85 & 0.0155 & 0.1804 & 0.00125 & 0.48181 & 1062 \\
\hline Kiowa_973_107.FIN2r & 169 & 0.04 & 0.0755 & 0.0011 & 1.889 & 0.0265 & 0.1821 & 0.0023 & 0.38859 & 1076 \\
\hline Kiowa-973_112.FIN2 & 100.9 & 1.07 & 0.0757 & 0.00065 & 1.917 & 0.018 & 0.1843 & 0.00125 & 0.45803 & 1084 \\
\hline Kiowa_973_7.FIN2 & 115.2 & 0.99 & 0.07596 & 0.000465 & 2.045 & 0.0155 & 0.1959 & 0.0013 & 0.65365 & 1129 \\
\hline Kiowa_973_27.FIN2 & 164.4 & 0.42 & 0.07791 & 0.000475 & 2.11 & 0.013 & 0.1966 & 0.001 & 0.4652 & 1151.9 \\
\hline Kiowa_973_9.FIN2 & 92.7 & 0.21 & 0.0798 & & 1.859 & 0.0315 & 0.173 & 0.0034 & 0.63259 & 1057 \\
\hline Kiowa_973_129.FIN2 & 334 & 0.92 & 0.08 & 0.00055 & 2.324 & 0.021 & 0.2101 & 0.00155 & 0.64788 & 1217 \\
\hline Kiowa_973_122.FIN2 & 154.7 & 1.65 & 0.08274 & 0.000485 & 2.408 & 0.02 & 0.2111 & 0.0016 & 0.76715 & 1242 \\
\hline Kiowa_973_31.FIN2 & 227 & 0.92 & 0.08389 & 0.000425 & 2.502 & 0.0195 & 0.2163 & 00175 & 0.87165 & 1270 \\
\hline Kiowa_973_95.FIN2 & 333 & 0.64 & 0.0846 & 0.0006 & 2.68 & 0.0195 & 0.2312 & 0.00185 & 0.62887 & 1321 \\
\hline Kiowa_973_35.FIN2 & 79.4 & 0.40 & 0.0887 & 0.0007 & 2.921 & 0.028 & 0.239 & 0.0024 & 0.61154 & 1383 \\
\hline Kiowa_973_107.FIN2c & 98.7 & 0.47 & 0.09 & 0.00055 & 3.068 & 0.018 & 0.2484 & 0.00125 & 0.38125 & 1423.7 \\
\hline Kiowa_973_101.FIN2 & 163.5 & 0.65 & 0.0904 & 0.00055 & 3.385 & 0.0245 & 0.2728 & 0.0019 & 0.61454 & 1498 \\
\hline Kiowa_973_26.FIN2 & $\begin{array}{r}243.8 \\
331\end{array}$ & 0.86 & 0.0902 & $\begin{array}{r}0.0006 \\
0.0075\end{array}$ & 3.122 & 0.0245 & 0.2508 & 0.00145 & 0.45729 & 1436 \\
\hline Kiowa_973_125.FIN2 & 331 & 0.38 & 0.0909 & 0.00075 & $\begin{array}{l}2.384 \\
3687\end{array}$ & 0.0305 & $\begin{array}{r}0.191 \\
02771\end{array}$ & 0.0027 & 0.79835 & 1234 \\
\hline $\begin{array}{l}\text { Kiowa_-973_139.FIN2 } \\
\text { Kolowa } 973 \text { 136 FIN2r }\end{array}$ & 115.9 & 0.51 & 0.0965 & 0.00085 & 3.687 & 0.0365 & 0.2771 & 0.0028 & 0.61053 & 1568 \\
\hline $\begin{array}{l}\text { Kiowa_973-136-FN2r } \\
\text { Kiowa 973 104.FIN2 }\end{array}$ & $\begin{array}{l}242 \\
507\end{array}$ & $\begin{array}{l}0.12 \\
0.41\end{array}$ & $\begin{array}{l}0.0981 \\
0.0992\end{array}$ & $\begin{array}{r}0.002 \\
0.00095\end{array}$ & $\begin{array}{r}3.11 \\
3.12\end{array}$ & $\begin{array}{r}0.1 \\
0.0365\end{array}$ & $\begin{array}{r}0.223 \\
0.2292\end{array}$ & $\begin{array}{r}0.0065 \\
0.00255\end{array}$ & $\begin{array}{l}0.761658 \\
0.69952\end{array}$ & $\begin{array}{l}\begin{array}{l}1400 \\
1432\end{array} \\
-\end{array}$ \\
\hline Kiowa_973_103.FIN2 & 956 & 0.02 & 0.1006 & 0.00135 & 2.781 & 0.046 & 0.2013 & 0.00295 & 0.58678 & 1346 \\
\hline Kiowa_973_40.FIN2 & 107.4 & 0.65 & 0.1009 & 0.00065 & 4.041 & 0.0305 & 0.2889 & 0.00205 & 0.5697 & 1640 \\
\hline Kiowa_973_93.FIN2 & 378 & 0.15 & 0.1028 & 0.00105 & 2.868 & 0.0365 & 0.2029 & 0.0024 & 0.66902 & 1369 \\
\hline Kiowa_973_73.FIN2 & 222.1 & 0.35 & 0.1042 & 0.001 & 3.94 & 0.0395 & 0.2738 & 0.00295 & 0.52125 & 1618 \\
\hline Kiowa 973 134.FIN2 & 193.9 & 0.95 & 0.1 & 0.00055 & 4.55 & 0.03 & 0.3228 & 0.00 & 0.73746 & 1746 \\
\hline Kiowa_973_48.FIN2 & 297 & 0.87 & & 0.00 & 4.3 & 0.0 & 0.304 & 0.00 & 0.69288 & 1702 \\
\hline Kiowa_973_62.FIN2 & 170 & 0.33 & & 0.0 & 4.6 & 0.02 & 0.3243 & 0.0 & 0.58834 & 1765 \\
\hline Kiowa_973_22.FIN2 & 158.2 & 0.36 & 0.1 & 0.0 & 4.28 & 0.02 & 0.295 & 0.00 & 0.53649 & 1689 \\
\hline Kiowa_973_124.FI & 72.4 & 1.69 & & 0.00085 & 4.8 & 0.0 & 0.33 & 0.00245 & 607 & 1790 \\
\hline Kiowa_973_81.FIN & 231.9 & 0.32 & 0.1068 & 0.00 & 4.80 & 0.033 & & 0.0021 & 0.69451 & 1785 \\
\hline Kiowa_973_94.FIN2 & 206.2 & 0.26 & 0.10722 & 0.00046 & 4.745 & 0.03 & & 0.002 & 0.79648 & 1773 \\
\hline Kiowa_973_65.FIN2 & 303 & 0.45 & & 0.0013 & 4.38 & 0.055 & 0.2973 & 0.0046 & 0.66519 & 1707 \\
\hline Kiowa_973_72.FIN2 & 240 & 0.40 & & 0.0009 & 3.451 & 0.045 & 0.2307 & 0.003 & 0.8269 & 1513 \\
\hline Kiowa_973_127.FIN2 & 536 & 0.03 & & 0.0009 & 3.66 & 0.05 & 0.2421 & 0.00255 & 0.78901 & 1556 \\
\hline Kiowa_973_24.FIN2 & 673 & 0.47 & 0.1099 & $\begin{array}{r}0.001 \\
0.017\end{array}$ & $\begin{array}{r}4.915 \\
5.19\end{array}$ & 0.042 & $\begin{array}{l}0.324 \\
0.336\end{array}$ & $\begin{array}{r}0.00355 \\
0.005\end{array}$ & $\begin{array}{l}0.55662 \\
0.51866\end{array}$ & $\begin{array}{l}1803 \\
1852\end{array}$ \\
\hline
\end{tabular}

$\begin{array}{rrrr}1.1 & 99 & 0.55 & 83 \\ 1.45 & 99.4 & 1.1 & 119 \\ 1.7 & 99.5 & 1.1 & 163 \\ 1.5 & 99.6 & 0.85 & 155 \\ 1.8 & 13.5 & 0.95 & 139 \\ 1.4 & 104.7 & 1.3 & 158 \\ 1.15 & 106.4 & 1.15 & 124 \\ 1.9 & 106.4 & 0.85 & 177 \\ 2.05 & 109.1 & 1.35 & 172 \\ 2.95 & 138.9 & 1.95 & 289 \\ 2.8 & 159.7 & 1.15 & 176 \\ 1.8 & 165 & 1.25 & 228 \\ 2.5 & 166.2 & 2.45 & 408 \\ 2 & 167 & 1.25 & 230 \\ 2.7 & 167.4 & 1.5 & 147 \\ 1.55 & 167.4 & 1.5 & 284 \\ 2.7 & 171.2 & 2.7 & 216 \\ 4.15 & 173 & 3.85 & 278 \\ 3.5 & 176.9 & 2.3 & 252 \\ 1.75 & 179.2 & 1.4 & 187 \\ 1.9 & 181.3 & 1.1 & 177 \\ 1.95 & 185.9 & 1.55 & 215 \\ 2.05 & 187.7 & 1.55 & 264 \\ 2.85 & 187.9 & 1.5 & 176 \\ 2.15 & 237 & 2.1 & 303 \\ 3.8 & 413.5 & 3.1 & 403 \\ 3.1 & 424.2 & 3.4 & 400 \\ 3.4 & 450.5 & 3.7 & 427 \\ 7 & 467 & 8 & 545 \\ 6 & 475 & 7 & 439 \\ .95 & 477.4 & 2.55 & 467 \\ 4.65 & 501.3 & 3.85 & 596 \\ 6.5 & 595 & 8 & 1026 \\ 3.45 & 612.4 & 3.6 & 631 \\ 6 & 622 & 5.5 & 596 \\ 5.5 & 659.8 & 4.9 & 608 \\ 6.5 & 697 & 10.5 & 667 \\ 6 & 945 & 5.5 & 914 \\ 5 & 1049 & 9 & 993 \\ 9 & 953 & 10 & 1034 \\ 5.5 & 1074 & 6.5 & 1053 \\ 7 & 904 & 10.5 & 1053 \\ 5.5 & 1069 & 7 & 1059 \\ 9.5 & 1078 & 12.5 & 1072 \\ 6 & 1090 & 6.5 & 1073 \\ 5 & 1153 & 7 & 1087 \\ 4.35 & 1157 & 5.5 & 1137 \\ 11.5 & 1025 & 18.5 & 1151 \\ 6.5 & 1229 & 8 & 191 \\ 6 & 1234 & 8.5 & 1257 \\ 6 & 1261 & 9 & 1285 \\ 5.5 & 1340 & 9.5 & 1298 \\ 7 & 1380 & 12.5 & 1396 \\ 4.55 & 1430 & 6.5 & 1425 \\ 5.5 & 1556 & 9.5 & 1426 \\ 6 & 1442 & 7.5 & 1427 \\ 9 & 1125 & 14.5 & 1440 \\ 8 & 1578 & 14 & 1552 \\ 24.5 & 1295 & 34 & 1573 \\ 9 & 1332 & 13 & 1598 \\ 12 & 1187 & 15 & 1630 \\ 6 & 1635 & 10.5 & 1641 \\ 9.5 & 1189 & 13 & 1666 \\ 8 & 1561 & 15.5 & 1683 \\ 5.5 & 1802 & 11.5 & 1683 \\ 5.5 & 1710 & 10 & 1691 \\ 4.95 & 1809 & 9 & 1172 \\ 5 & 1665 & 10 & 1717 \\ 8 & 1850 & 11.5 & 1725 \\ 5.5 & 1827 & 10 & 1743 \\ 5.5 & 1797 & 9.5 & 1750 \\ 11 & 1672 & 22.5 & 1756 \\ 10 & 1336 & 16 & 1784 \\ 11.5 & 1396 & 13 & 1785 \\ 77 & 1807 & 17.5 & 1791 \\ 13 & 1867 & 23.5 & 1838 \\ & & & \end{array}$

\begin{tabular}{|c|c|c|}
\hline 25.5 & 99.0 & 0.6 \\
\hline 33 & 99.4 & 1.1 \\
\hline 38 & 99.5 & 1.1 \\
\hline & 99.6 & 0.9 \\
\hline 36.5 & 103.5 & 1.0 \\
\hline $\begin{array}{r}29 \\
225\end{array}$ & 104.7 & 1.3 \\
\hline $\begin{array}{r}22.5 \\
37\end{array}$ & 106.4 & 1.2 \\
\hline & & 1.4 \\
\hline 41 & 138.9 & 2.0 \\
\hline 38.5 & 159.7 & 1.2 \\
\hline & 165.0 & 1.3 \\
\hline 25.5 & 166.2 & 2.5 \\
\hline 25.5 & 167.0 & 1.3 \\
\hline & 167.4 & 1.5 \\
\hline 18.5 & 167.4 & 1.5 \\
\hline 40.5 & 171.2 & 2.7 \\
\hline 49 & 173.0 & 3.9 \\
\hline 42 & 176.9 & 2.3 \\
\hline 26 & 179.2 & 1.4 \\
\hline 22 & 181.3 & 1.1 \\
\hline 22 & $\begin{array}{l}185.9 \\
1877\end{array}$ & 1.6 \\
\hline & $\begin{array}{l}187.7 \\
1879\end{array}$ & $\begin{array}{l}1.6 \\
15\end{array}$ \\
\hline 33.5 & $\begin{array}{l}187.9 \\
2370\end{array}$ & $\begin{array}{l}1.5 \\
2 .\end{array}$ \\
\hline $\begin{array}{l}11 \\
22\end{array}$ & $\begin{array}{l}237.0 \\
4135\end{array}$ & $\begin{array}{l}2.1 \\
3.1\end{array}$ \\
\hline 20.5 & 424.2 & 3.4 \\
\hline & 450.5 & 3.7 \\
\hline 40.5 & 467.0 & 8.0 \\
\hline & 475.0 & 7.0 \\
\hline 17.5 & 477.4 & 2.6 \\
\hline 25.5 & 501.3 & 3.9 \\
\hline 26 & 595.0 & 8.0 \\
\hline & 612.4 & 3.6 \\
\hline 30.5 & 622.0 & 5.5 \\
\hline $\begin{array}{l}23.5 \\
27\end{array}$ & 659.8 & $\begin{array}{r}4.9 \\
105\end{array}$ \\
\hline 20.5 & 914.0 & $\begin{array}{l}0.5 \\
20.5\end{array}$ \\
\hline 14 & 993.0 & 14.0 \\
\hline 30 & 1034.0 & 30.0 \\
\hline 17.5 & 1053.0 & 17.5 \\
\hline 14 & 1053.0 & 14.0 \\
\hline 16 & 1059.0 & 16.0 \\
\hline 29.5 & 1072.0 & 29.5 \\
\hline 17.5 & 1073.0 & 17.5 \\
\hline 12.5 & 1087.0 & 12.5 \\
\hline $\begin{array}{l}12 \\
33\end{array}$ & $\begin{array}{l}1137.0 \\
11510\end{array}$ & 12.0 \\
\hline 14 & $\begin{array}{l}1151.0 \\
\end{array}$ & $\begin{array}{l}33.0 \\
14.0\end{array}$ \\
\hline 11.5 & 1257.0 & 11.5 \\
\hline 10 & 1285.0 & 10.0 \\
\hline 13.5 & 1298.0 & 13.5 \\
\hline 14.5 & 1396.0 & 14.5 \\
\hline 11.5 & 1425.0 & 11.5 \\
\hline 12 & 1426.0 & 12.0 \\
\hline 13 & 1427.0 & 13.0 \\
\hline 16.5 & 1440.0 & 16.5 \\
\hline 17.5 & 1552.0 & 17.5 \\
\hline 37.5 & 1573.0 & 37.5 \\
\hline 17 & 1598.0 & 17.0 \\
\hline 25.5 & 1630.0 & 25.5 \\
\hline 12.5 & 1641.0 & 12.5 \\
\hline 19 & 1662.0 & 19.0 \\
\hline 18 & 1683.0 & 18.0 \\
\hline 9.5 & 1683.0 & 9.5 \\
\hline 9.5 & 1691.0 & 9.5 \\
\hline 10 & 1712.0 & 10.0 \\
\hline 12.5 & 1717.0 & 12.5 \\
\hline $\begin{array}{l}14.5 \\
1.5\end{array}$ & 1725.0 & 14.5 \\
\hline 10.5 & 1743.0 & 10.5 \\
\hline 8 & $1 / 50.0$ & 8.0 \\
\hline 22 & 1750.0 & 22.0 \\
\hline 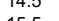 & 11804.0 & 14.5 \\
\hline 10.5 & 1180.0 & 15.5 \\
\hline $\begin{array}{l}10.0 \\
27.5\end{array}$ & 18380 & $\begin{array}{r}10.5 \\
27.5\end{array}$ \\
\hline
\end{tabular}

Rim 


\begin{tabular}{|c|c|c|c|c|c|c|c|c|c|c|c|c|c|c|c|c|c|c|c|}
\hline Kiowa_973_136.FIN2C & 82.3 & 0.36 & 0.1138 & 0.00105 & 5.69 & 0.06 & 0.3638 & 0.0036 & 0.57267 & 1926 & 9 & 1998 & 17 & 1851 & 17 & 1851.0 & 17.0 & -7.9 & Core \\
\hline Kiowa_973_78.FIN2 & 104.7 & 0.11 & 0.1141 & 0.00075 & 3.995 & 0.0405 & 0.2548 & 0.0024 & 0.71914 & 1628 & 8 & 1461 & 12.5 & 1861 & 12.5 & 1861.0 & 12.5 & 21.5 & \\
\hline Kiowa_973_113.FIN2 & 347 & 0.35 & 0.1147 & 0.00095 & 5.25 & 0.05 & 0.3336 & 0.0036 & 0.80907 & 1856 & 9 & 1852 & 17.5 & 1867 & 15 & 1867.0 & 15.0 & 0.8 & \\
\hline Kiowa_973_18.FIN2 & 362 & 0.38 & 0.1147 & 0.00065 & 5.44 & 0.0385 & 0.3446 & 0.00255 & 0.6861 & 1889 & 6 & 1907 & 12 & 1870 & 10.5 & 1870.0 & 10.5 & -2.0 & \\
\hline Kiowa_973_100.FIN2 & 976 & 0.60 & 0.1178 & 0.0015 & 4.698 & 0.0335 & 0.2956 & 0.0036 & 0.44446 & 1764 & 6 & 1666 & 18 & 1899 & 21 & 1899.0 & 21.0 & 12.3 & \\
\hline Kiowa_973_51.FIN2 & 41.58 & 0.71 & 0.117 & 0.00115 & 5.51 & 0.08 & 0.3409 & 0.00415 & 0.81822 & 1896 & 12.5 & 1889 & 20 & 1902 & 18 & 1902.0 & 18.0 & 0.7 & \\
\hline Kiowa_973_92.FIN2 & 214 & 0.50 & 0.1186 & 0.00135 & 5.5 & 0.06 & 0.3406 & 0.0043 & 0.54624 & 1905 & 10.5 & 1890 & 20.5 & 1920 & 20.5 & 1920.0 & 20.5 & 1.6 & \\
\hline Kiowa_973_131.FIN2 & 32.4 & 0.58 & 0.1192 & 0.0014 & 5.81 & 0.12 & 0.356 & 0.0075 & 0.85194 & 1932 & 19.5 & 1955 & 37.5 & 1932 & 22 & 1932.0 & 22.0 & -1.2 & \\
\hline Kiowa_973_98.FIN2 & $\begin{array}{r}143.5 \\
81\end{array}$ & 0.58 & 0.1554 & 0.0009 & 9.51 & 0.08 & 0.4448 & $\begin{array}{r}0.0034 \\
0.00425\end{array}$ & 0.76606 & 2384 & 8 & 2369 & 15 & 2402 & 9.5 & 2402.0 & 9.5 & 1.4 & \\
\hline $\begin{array}{l}\text { Kiowa_973_29.FIN2 } \\
\text { Kiowa } 973 \text { 43.FIN2 }\end{array}$ & $\begin{array}{r}81 \\
83.4\end{array}$ & $\begin{array}{l}0.72 \\
0.97\end{array}$ & $\begin{array}{l}0.1646 \\
0.1896\end{array}$ & $\begin{array}{l}0.0009 \\
0.0011\end{array}$ & $\begin{array}{r}11.9 \\
13.24\end{array}$ & 0.11 & 0.5238 & 0.00425 & 0.80991 & 2591 & $\begin{array}{r}9 \\
75\end{array}$ & 2711 & 18 & 2499 & 9.5 & 2499.0 & 9.5 & -8.5 & \\
\hline $\begin{array}{l}\text { Kiowa_973_43.FIN2 } \\
\text { Kiowa 973 11:FN2 }\end{array}$ & 106.3 & 0.97 & 0.1896 & $\begin{array}{l}0.00011 \\
0.0011\end{array}$ & 13.24 & $\begin{array}{r}0.11 \\
0.125\end{array}$ & $\begin{array}{l}0.5073 \\
0.263\end{array}$ & $\begin{array}{l}0.0041 \\
0.005\end{array}$ & $\begin{array}{l}0.75465 \\
0.9483\end{array}$ & 2699 & $\begin{array}{r}7.5 \\
17.5\end{array}$ & 2641 & $\begin{array}{l}17.5 \\
25.5\end{array}$ & 2734 & 9.5 & 2734.0 & $\begin{array}{l}9.5 \\
-15\end{array}$ & 3.4 & \\
\hline Kiowa 973-19.FIN2 & 33.2 & 0.11 & 0.1611 & 0.00135 & 5.75 & 0.055 & $\begin{array}{r}0.263 \\
0.2594\end{array}$ & 0.00185 & 0.55489 & $\begin{array}{l}2038 \\
1934\end{array}$ & $\begin{array}{r}71.5 \\
8.5\end{array}$ & $\begin{array}{l}7497 \\
1486\end{array}$ & $\begin{array}{r}25.5 \\
9.5\end{array}$ & $\begin{array}{l}2672 \\
2456\end{array}$ & $\begin{array}{l}70 ! \\
14 ! \\
\text { ! }\end{array}$ & $\begin{array}{l}\text { DISCARD } \\
\text { DISCARD }\end{array}$ & $\begin{array}{l}\text { \#VAUE! } \\
\text { \#YAUEI }\end{array}$ & $\begin{array}{l}44.0 \\
33.5\end{array}$ & \\
\hline Kiowa_973 32 .FIN2G & 272 & 0.60 & 0.0767 & 0.0011 & 0.524 & 0.008 & 0.0498 & 0.00055 & 0.55317 & 427 & 5.5 & 313.1 & 3.3 & 1101 & 28 & DISCARD & \#VALUE! & 26.7 & Gore \\
\hline Kiowa_973_53.FIN2 & 454 & 0.43 & 0.1089 & 0.00125 & 2.882 & 0.04 & 0.1925 & 0.00255 & 0.66874 & 1371 & 10.5 & 1133 & 14 & 1757 & 21.5 & DISCARD & \#VALUE! & 35.5 & \\
\hline Kiowa-973_61.FIN2 & 14.9 & 7.85 & 0.222 & 0.0065 & 8.19 & 0.29 & 0.2611 & 0.00305 & 0.56265 & 2190 & 32 & 1493 & 15.5 & 2890 & $48.5 !$ & DISCARD & \#VALUE! & 48.3 & \\
\hline Kiowa-973_91.FIN2 & 17.5 & 0.71 & 0.122 & 0.0033 & 1.63 & 0.05 & 0.0975 & 0.002 & 0.50456 & 958 & 20 & 598 & 11.5 & 1900 & 50 & DISCARD & \#VALUE! & 37.6 & \\
\hline Kiowa_973_110.FIN2 & 1820 & 0.21 & 0.0754 & 0.00185 & 0.1257 & 0.00295 & 0.01218 & 0.000175 & 0.14146 & 119.7 & 2.65 & 78.1 & 1.1 & 1003 & 46.5 & DISCARD & \#VALUE! & 34.8 & \\
\hline Kiowa_-973_-120.FIN2 & 324.9 & 0.60 & 0.1313 & 0.00105 & 2.895 & 0.026 & 0.1605 & 0.00145 & 0.55402 & 1380 & 7 & 959 & 8 & 2113 & 14.5 & BISCARD & \#VALUE! & 54.6 & \\
\hline e Name: Kiowa-1200 & & & & internal & & internal & & internal & & 207/235 & internal & $206 / 238$ & internal & 7/206 & internal & Best age & internal & & \\
\hline Analysis ID & \begin{tabular}{|l|}
$\mathrm{U}] \mathrm{ppm}$ \\
\end{tabular} & \begin{tabular}{|l|l} 
Th/U \\
\end{tabular} & $207 / 206$ & 10 error & 207/235 & $1 \sigma$ error & 206/238 & $1 \sigma$ error & RHO & Age Ma & 10 error & Age (Ma) & 10 error & Age (Ma) & $1 \sigma$ error & (Ma) & $1 \sigma$ error & \% Discordance ${ }^{*}$ & Rim/Core \\
\hline KIOWA_1200_39.FIN2r & 246 & 0.09 & 0.0492 & 0.00295 & 0.0694 & 0.00445 & 0.00985 & 0.00031 & -0.11012 & 67.9 & 4.2 & 63.2 & 2 & 150 & 115 & 63.2 & 2.0 & 6.9 & Rim \\
\hline KIOWA_-1200_96.FIN2 & 78.3 & 0.87 & 0.049 & 0.00245 & 0.0669 & 0.0034 & 0.01008 & 0.000165 & 0.26202 & 65.7 & 3.25 & 64.6 & 1.05 & 150 & 85 & 64.6 & 1.1 & 1.7 & \\
\hline KIOWA_1200_61.FIN2 & 353.3 & 0.23 & 0.0485 & 0.00135 & 0.068 & 0.0019 & 0.01021 & 0.000115 & 0.096821 & 67 & 1.75 & 65.5 & 0.7 & 120 & 50 & 65.5 & 0.7 & 2.2 & \\
\hline KIOWA_1200_82.FIN2 & 89 & 0.60 & 0.0467 & 0.00285 & 0.0653 & 0.0038 & 0.01054 & 0.00016 & -0.0466 & 63.4 & 3.6 & 67.6 & 1 & 20 & 100 & 67.6 & 1.0 & -6.6 & \\
\hline KIOWA_1200_89.FIN2 & 262 & 1.00 & 0.0561 & 0.002 & 0.084 & 0.00285 & 0.01089 & 0.00012 & 0.11761 & 81.4 & 2.7 & 69.8 & 0.75 & 410 & 70 & 69.8 & 0.8 & 14.3 & \\
\hline KIOWA_1200_84.FIN2r & 274 & 0.27 & 0.0487 & 0.00215 & 0.0707 & 0.0033 & 0.01095 & 0.00014 & 0.24914 & 69.1 & 3.15 & 70.2 & 0.9 & 160 & 85 & 70.2 & 0.9 & -1.6 & Rim \\
\hline KIOWA_1200_85.FIN2 & 113.9 & 0.42 & 0.0429 & 0.0024 & 0.0652 & 0.00345 & 0.01122 & 0.000175 & 0.021525 & 63.5 & 3.3 & 71.9 & 1.1 & -110 & 90 & 71.9 & 1.1 & -13.2 & \\
\hline KIOWA_1200_95.FIN2 & 167 & 0.85 & 0.0455 & 0.00185 & 0.0696 & 0.0027 & 0.01138 & 0.000135 & 0.038178 & 67.9 & 2.55 & 73 & 0.85 & 0 & 70 & 73.0 & 0.9 & -7.5 & \\
\hline KIOWA_1200_73.FIN2r & 203.9 & 0.57 & 0.048 & 0.0035 & 0.079 & 0.0065 & 0.01191 & 0.000455 & 0.35053 & 77 & 6 & 76.3 & 2.9 & 100 & 140 & 76.3 & 2.9 & 0.9 & $\operatorname{Rim}$ \\
\hline KIOWA_1200_26.FIN2r & 887 & 0.71 & 0.0489 & 0.0016 & 0.0821 & 0.0025 & 0.01255 & 0.00013 & -0.054063 & 80 & 2.35 & 80.4 & 0.85 & 140 & 65 & 80.4 & 0.9 & -0.5 & $\operatorname{Rim}$ \\
\hline KIOWA_1200_1.FIN2 & 233 & 0.59 & 0.0508 & 0.0016 & 0.0869 & 0.0025 & 0.01277 & 0.000125 & -0.020326 & 85.2 & 2.3 & 81.8 & 0.8 & 190 & 60 & 81.8 & 0.8 & 4.0 & \\
\hline KIOWA_1200_127.FIN2 & 396 & 0.70 & 0.0516 & 0.0011 & 0.0972 & 0.00215 & 0.01352 & 0.00015 & 0.23255 & 93.9 & 2 & 86.5 & 0.95 & 235 & 42.5 & 86.5 & 1.0 & 7.9 & \\
\hline KIOWA_1200_63.FIN2 & 1154 & 0.05 & 0.0494 & 0.0007 & 0.0923 & 0.00115 & 0.0137 & 0.00012 & 0.24001 & 89.6 & 1.1 & 87.7 & 0.75 & 166 & 28.5 & 87.7 & 0.8 & 2.1 & \\
\hline KIOWA_1200_66.FIN2 & 921 & 0.51 & 0.0481 & 0.0008 & 0.0919 & 0.00155 & 0.01399 & 0.000105 & 0.28098 & 89.4 & 1.45 & 89.5 & 0.65 & 113 & 32.5 & 89.5 & 0.7 & -0.1 & \\
\hline KIOWA_1200_133.FIN2 & 141.1 & 0.85 & 0.0473 & 0.00185 & 0.0915 & 0.0034 & 0.01421 & 0.00016 & -0.030302 & 88.2 & $\begin{array}{l}3.15 \\
155\end{array}$ & 90.9 & 1 & $\begin{array}{r}90 \\
902\end{array}$ & 70 & 90.9 & 1.0 & -3.1 & \\
\hline $\begin{array}{l}\text { KIIOWA_1200_91.FIN2 } \\
\text { KOWA_ 1200 54FIN }\end{array}$ & $\begin{array}{l}466 \\
898\end{array}$ & 1.07 & $\begin{array}{l}0.0501 \\
0.0054\end{array}$ & $\begin{array}{l}0.00085 \\
0.00075\end{array}$ & 0.0977 & $\begin{array}{l}0.00165 \\
0.00145\end{array}$ & 0.01437 & 0.000105 & 0.22057 & $\begin{array}{c}94.5 \\
08.8\end{array} \mathrm{C}$ & $\begin{array}{l}1.55 \\
1.35\end{array}$ & 92 & 0.7 & 192 & 34.5 & 92.0 & 0.7 & 2.6 & \\
\hline $\begin{array}{l}\text { KKOWA_1200_54.FIN2 } \\
\text { KIOWA 1 100 57 FIN }\end{array}$ & 898 & $\begin{array}{l}0.46 \\
0.43\end{array}$ & $\begin{array}{l}0.0504 \\
0.0486\end{array}$ & $\begin{array}{l}0.00075 \\
0.00095\end{array}$ & $\begin{array}{l}0.1023 \\
0.0988\end{array}$ & $\begin{array}{l}0.00145 \\
0.00185\end{array}$ & $\begin{array}{l}0.01444 \\
0.01465\end{array}$ & $\begin{array}{l}0.000095 \\
0.000115\end{array}$ & 0.14165 & 98.8 & 1.35 & $\begin{array}{l}92.4 \\
93.8\end{array}$ & 0.6 & 222 & 32 & 92.4 & 0.6 & 6.5 & \\
\hline KIOWA_1200_120.FIN2 & $\begin{array}{r}4871 \\
487.5\end{array}$ & $\begin{array}{l}0.43 \\
0.31\end{array}$ & $\begin{array}{l}0.0486 \\
0.0493\end{array}$ & $\begin{array}{l}0.000009 \\
0.009\end{array}$ & $\begin{array}{l}0.0988 \\
0.0995\end{array}$ & $\begin{array}{r}0.00185 \\
0.0017\end{array}$ & $\begin{array}{l}0.01465 \\
0.01465\end{array}$ & 0.000125 & $\begin{array}{l}0.12355 \\
0.13719\end{array}$ & $\begin{array}{l}95.5 \\
96.2\end{array}$ & $\begin{array}{l}1.75 \\
1.55\end{array}$ & $\begin{array}{l}93.8 \\
93.8\end{array}$ & $\begin{array}{r}0.15 \\
0.8\end{array}$ & $\begin{array}{l}134 \\
155\end{array}$ & $\begin{array}{l}39.5 \\
36.5\end{array}$ & $\begin{array}{l}93.8 \\
93.8\end{array}$ & 0.8 & 2.8 & \\
\hline KIOWA_1200_38.FIN2 & 499 & 0.40 & 0.0485 & 0.001 & 0.0984 & 0.0018 & 0.01467 & 0.00011 & -0.11608 & 95.1 & 1.65 & 93.9 & 0.7 & 137 & 40 & 93.9 & 0.7 & 1.3 & \\
\hline KIOWA_1200_100.FIN2 & 996 & 0.59 & 0.0478 & 0.00055 & 0.0975 & 0.00115 & 0.01481 & 0.000085 & 0.15266 & 94.4 & 1.1 & 94.7 & 0.55 & 92 & 25 & 94.7 & 0.6 & -0.3 & \\
\hline KIOWA_1200_106.FIN2 & 641 & 0.56 & 0.0479 & 0.0009 & 0.1032 & 0.00195 & 0.01564 & 0.000115 & 0.18288 & 99.6 & 1.8 & 100 & 0.7 & 100 & 38 & 100.0 & 0.7 & -0.4 & \\
\hline KIOWA_1200_132.FIN2 & 429 & 0.77 & 0.0494 & 0.0009 & 0.1055 & 0.0018 & 0.01563 & 0.000135 & 0.11798 & 101.7 & 1.65 & 100 & 0.85 & 155 & 36.5 & 100.0 & 0.9 & 1.7 & \\
\hline KIOWA_1200_15.FIN2 & 211 & 0.53 & 0.0462 & 0.0011 & 0.1494 & 0.0034 & 0.02322 & 0.000185 & 0.071411 & 140.8 & 3 & 148 & 1.2 & 62 & 44 & 148.0 & 1.2 & -5.1 & \\
\hline KIOWA_1200_134.FIN2 & 74 & 1.02 & 0.0486 & 0.0023 & 0.159 & 0.0065 & 0.02326 & 0.00043 & 0.096905 & 148 & 6 & 148.2 & 2.7 & 110 & 80 & 148.2 & 2.7 & -0.1 & \\
\hline KIOWA_1200_104.FIN2 & 39.5 & 1.38 & 0.0527 & 0.00285 & 0.169 & 0.0095 & 0.02335 & 0.000375 & 0.064528 & 157 & 8 & 149.2 & 2.35 & 270 & 100 & 149.2 & 2.4 & 5.0 & \\
\hline KIOWA-1200_25.FIN2 & 1411 & 0.33 & 0.0487 & 0.000445 & 0.1704 & 0.0015 & 0.02553 & 0.000095 & 0.096601 & 159.6 & 1.3 & 162.5 & 0.6 & 130 & 19.5 & 162.5 & 0.6 & -1.8 & \\
\hline KIOWA_1200_48.FIN2 & 532 & 1.14 & 0.0478 & 0.0007 & 0.1707 & 0.00255 & 0.02574 & 0.000125 & 0.14763 & 160.6 & 2.2 & 164 & 0.75 & 101 & 29.5 & 164.0 & 0.8 & -2.1 & \\
\hline KIOWA_1200_105.FIN2 & 271.6 & 0.48 & 0.0488 & 0.001 & 0.1718 & 0.0033 & 0.02587 & 0.000175 & 0.023321 & 160.5 & 2.85 & 164.6 & 1.1 & 129 & 40 & 164.6 & 1.1 & -2.6 & \\
\hline KIOWA_-1200_70.FIN2r & 96.2 & 0.99 & 0.0476 & 0.0029 & 0.174 & 0.0105 & 0.0259 & 0.00065 & 0.086794 & 161 & 9 & 164.8 & 3.95 & 110 & 115 & 164.8 & 4.0 & -2.4 & $\operatorname{Rim}$ \\
\hline KIOWA_-1200_29.FIN2 & 487 & 1.54 & 0.0492 & 0.0007 & 0.1758 & 0.00235 & 0.026 & 0.000145 & 0.034111 & 164.6 & 2 & 165.6 & 0.9 & 173 & 29 & 165 & 0.9 & -0.6 & \\
\hline KIOWA_1200_126.FIN2 & 806 & 1.07 & 0.0498 & 0.0006 & 0.1806 & 0.00195 & 0.02631 & 0.000195 & 0.1828 & 168.4 & 1.65 & 167.4 & 1.2 & 178 & 26 & 167 & 1.2 & 0.6 & \\
\hline KIOWA_1200_43.FIN2 & 424 & 0.7 & 0.0507 & 0.0007 & 894 & 0.0026 & 0.02676 & 0.00018 & 0.18879 & 175. & 2.2 & 170.2 & 1.15 & 220 & 30 & 170 & 1.2 & 3.2 & \\
\hline KIOWA_1200_63a.FIN2r & 204.7 & 0.8 & 0.0481 & 0.00135 & & 0.00465 & 0.02 & 00034 & 0.081923 & 165. & 4 & 171.5 & 2.15 & 100 & 55 & 171 & 2.2 & -3.6 & Rim \\
\hline KIOWA_1200_74.FIN2 & & & 0.0494 & 0.00 & & 0.00225 & 0.02737 & 0.00018 & 0.18495 & 174. & 1.95 & 174.1 & 1.15 & 161 & 26 & & 1.2 & 0.3 & \\
\hline KIOWA_1200_103.FIN2 & 729 & 0.58 & 0.0493 & 0.00055 & 0. & 0.0022 & 0.02775 & 0.00017 & 0.20873 & 17 & 1.85 & 176.5 & 1.05 & 161 & 23.5 & 176 & 1.1 & 0.3 & \\
\hline KIOWA_1200_102.FIN2 & 752 & 0. & 0.0504 & & 2114 & 0.0022 & 0.03034 & 0.000155 & 0.33658 & 194.5 & 1.85 & 192.7 & 0.95 & 213 & 22 & 192 & 1.0 & 0.9 & \\
\hline KIOWA_1200_32.FIN2 & 349.2 & 0.30 & 0.0507 & & 0.2208 & 0.0033 & 0.03167 & 0.00019 & 0.1049 & 203.3 & 2.75 & & 1.2 & 211 & 32.5 & 201 & 1.2 & 1.1 & \\
\hline KIOWA_1200_99.FIN2 & 524 & 0.53 & 0.0498 & 0.0009 & 0.2507 & 0.0035 & 0.0373 & 0.00055 & 0.26003 & 227.2 & 2.8 & 236 & 3.35 & 174 & 35.5 & 236.0 & 3.4 & -3.9 & \\
\hline 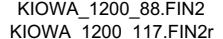 & $\begin{array}{r}1050 \\
614\end{array}$ & $\begin{array}{l}0.45 \\
0.64\end{array}$ & $\begin{array}{l}0.0515 \\
0.0559\end{array}$ & $\begin{array}{l}0.0005 \\
0.001\end{array}$ & $\begin{aligned} 0.2843 \\
0.342\end{aligned}$ & $\begin{array}{l}0.00255 \\
0.005\end{array}$ & $\begin{array}{l}0.04044 \\
0.04429\end{array}$ & $\begin{array}{l}0.000215 \\
0.00037\end{array}$ & $\begin{array}{l}0.066167 \\
0.1075\end{array}$ & $\begin{array}{l}254 \\
298\end{array}$ & $\begin{array}{r}2 \\
385\end{array}$ & $\begin{array}{l}256.2 \\
2793\end{array}$ & $\begin{array}{l}1.35 \\
2.3\end{array}$ & $\begin{array}{l}254 \\
433\end{array}$ & $\begin{array}{r}22 \\
395\end{array}$ & $\begin{array}{l}256.2 \\
2793\end{array}$ & 1.4 & -0.9 & Rim \\
\hline KIOWA 1200 37.FIN2 & 187 & 0.84 & 0.0548 & 0.00085 & 0.425 & 0.007 & 0.05641 & 0.000465 & 0.29046 & 360 & 5 & 353.7 & 2.85 & 384 & 33.5 & 353.7 & 2.9 & $\begin{array}{l}6.3 \\
1.8\end{array}$ & RIm \\
\hline KIOWA 1200 117.FIN2C & 353 & 0.74 & 0.059 & 0.00085 & 0.626 & 0.009 & 0.0757 & 0.0008 & 0.51839 & 493 & 5.5 & 470.4 & 4.75 & 550 & 32.5 & 470.4 & 4.8 & 4.6 & Core \\
\hline KIOWA_1200_49.FIN2 & 81.8 & 0.53 & 0.0652 & 0.00135 & 0.773 & 0.016 & 0.0847 & 0.00075 & 0.19546 & 578 & 9.5 & 524.1 & 4.35 & 756 & 45.5 & 524.1 & 4.4 & 9.3 & \\
\hline KIOWA_1200_3.FIN2 & 305 & 0.93 & 0.06 & 0.00055 & 0.803 & 0.0075 & 0.0973 & 0.0006 & 0.30639 & 598.2 & 4.2 & 599.4 & 3.45 & 607 & 20.5 & 599.4 & 3.5 & -0.2 & \\
\hline KIOWA_1200_121.FIN2 & 228 & 0.93 & 0.0621 & 0.00 & 0.897 & 0.01 & 0.1049 & 0.0008 & 0.43486 & 653 & 5 & 643 & 4.7 & 683 & 23.5 & 643.0 & 4.7 & 1.5 & \\
\hline KIOWA_1200_55.FIN2 & 240 & 0.26 & 0.0632 & 0.0006 & 1.023 & 0.016 & 0.1168 & 0.00125 & 0.72669 & 713 & 8.5 & 713 & 7 & 711 & 20 & 713.0 & 7.0 & 0.0 & \\
\hline KIOWA_1200_77.FIN2 & 147.1 & 0.46 & 0.0718 & 0.00 & 1.634 & 0.0145 & 0.1642 & 0.00075 & 0.28891 & 982 & 5.5 & 979.8 & 4.2 & 985 & 17.5 & 985.0 & 17.5 & 0.5 & \\
\hline KIOWA_1200_8.FIN2 & 422 & 0.1 & 0.0739 & 0.00 & 1.71 & 0.012 & 0.1687 & 0.00085 & 0.38403 & 1011.9 & 4.35 & 1004.6 & 4.7 & 1030 & 14 & 1030.0 & 14.0 & 2.5 & \\
\hline KIOWA_1200_108.FIN2r & 606 & 0.24 & 0.0742 & 0.00125 & 1.78 & 0.044 & 0.1 & 0.0037 & 0.7276 & 1037 & 16 & 1044 & 20.5 & 1042 & 34.5 & 1042.0 & 34.5 & -0.2 & $\operatorname{Rim}$ \\
\hline KIOWA_1200_27.FIN2 & 35.2 & 1.0 & 0.0751 & 0.00115 & 1.9 & 0.0325 & 0.1919 & 0.0017 & 0.34413 & 111 & 10.5 & 1131 & 9.5 & 1055 & 31.5 & 1055.0 & 31.5 & -7.2 & \\
\hline KIOWA_1200_113.FIN2 & 43 & 0.67 & 0.0765 & 0.00125 & 1.8 & 0.027 & 0.1777 & 0.00125 & 0.15801 & 1071 & 9.5 & 1054 & 7 & 1097 & 33 & 1097.0 & 33.0 & 3.9 & \\
\hline KIOWA-1200_123.FIN2 & 115.3 & 0.44 & 0.0773 & 0.00085 & 2.072 & 0.021 & 0.1936 & 0.0014 & 0.23161 & 1138 & 7 & 1140 & 7.5 & 1119 & 22 & 1119.0 & 22.0 & -1.9 & \\
\hline KIOWA_-1200_135.FIN2 & 100 & 0.60 & 0.0781 & 0.0008 & 2.114 & 0.02 & 0.1955 & 0.0011 & 0.21825 & 1152 & 6.5 & 1150 & 6 & 1135 & 20.5 & 1135.0 & 20.5 & -1.3 & \\
\hline
\end{tabular}




\begin{tabular}{|c|c|c|c|c|c|c|c|c|c|c|}
\hline KIOWA_1200_125.FIN2 & 299.1 & 0.40 & 0.0776 & 0.0005 & 2.118 & 0.014 & 0.1954 & 0.001 & 0.31544 & 1154.3 \\
\hline KIOWA_1200_11.FIN2 & 134.2 & 0.94 & 0.0789 & 0.00115 & 1.787 & 0.0255 & 0.1654 & 0.0011 & 0.20394 & 1040 \\
\hline KIOWA_1200_110.FIN2 & 113.7 & 0.99 & 0.0792 & 0.0008 & 2.202 & 0.0195 & 0.2001 & 0.00145 & $\begin{array}{r}0.2859 \\
0.5039\end{array}$ & 1183 \\
\hline KIOWA_1200_101.FIN2 & 730 & 0.69 & 0.07906 & 0.00039 & 2.225 & 0.012 & 0.2027 & 0.00105 & 0.50395 & 1188 \\
\hline KIOWA_1200_92.FIN2 & 1024 & 0.47 & 0.07999 & 0.000325 & 2.065 & 0.0115 & 0.1878 & 0.0008 & 0.62438 & 1136.4 \\
\hline KIOWA_1200_68.FIN2 & 86.4 & 0.82 & 0.0803 & 0.00085 & 2.344 & 0.0235 & 0.2125 & 0.0015 & 0.31407 & 1229 \\
\hline KIOWA_1200_46.FIN2 & 376 & 0.27 & 0.0805 & 0.0006 & 2.324 & 0.016 & 0.2066 & 0.0013 & 0.43337 & 1219 \\
\hline IIOWA_1200_67.FIN2 & 89.6 & 0.71 & 0.0808 & 0.00075 & 2.145 & 0.0185 & 0.1926 & 0.0012 & 0.28059 & 1164 \\
\hline $\begin{array}{l}\text { IIOWA_1200_19.FN2 } \\
\text { KIOWA 100 8.FIN2 }\end{array}$ & $\begin{array}{l}76.8 \\
55.6\end{array}$ & 0.88 & 0.0805 & $\begin{array}{l}0.00115 \\
0.00095\end{array}$ & $\begin{array}{l}1.942 \\
2298 \\
2298\end{array}$ & 0.0265 & $\begin{array}{l}0.1775 \\
0.2069\end{array}$ & 0.00115 & 0.28563 & 1090 \\
\hline $\begin{array}{l}\text { KIIWA-1200_83.FIN2 } \\
\text { KIOWA 1200 128.FIN2 }\end{array}$ & $\begin{array}{l}55.6 \\
78.8\end{array}$ & 0.61 & 0.0806 & $\begin{array}{r}0.00095 \\
0.0008\end{array}$ & 2.298 & 0.0255 & 0.2069 & 0.0018 & 0.32396 & 1208 \\
\hline KIOWA 1200 69.FIN2 & $\begin{array}{l}78.8 \\
512\end{array}$ & $\begin{array}{l}0.75 \\
0.53\end{array}$ & $\begin{array}{r}0.081 \\
0.08565\end{array}$ & $\begin{array}{l}0.0008 \\
0.00047\end{array}$ & $\begin{array}{l}2.213 \\
2.685\end{array}$ & $\begin{array}{l}0.0205 \\
0.0165\end{array}$ & $\begin{array}{l}0.1983 \\
0.2275\end{array}$ & $\begin{array}{l}0.00144 \\
0.00125\end{array}$ & $\begin{array}{l}0.24323 \\
0.47989\end{array}$ & $\begin{array}{r}11184 \\
1324.7\end{array}$ \\
\hline KIOWA_1200_44.FIN2 & 2490 & 0.30 & 0.08636 & 0.000335 & 3.188 & 0.016 & 0.2638 & 0.00125 & 0.71141 & 1455.2 \\
\hline KIOWA_1200_119.FIN2 & 676 & 0.80 & 0.08683 & 0.000425 & 2.648 & 0.034 & 0.2168 & 0.0021 & 0.93505 & 1314 \\
\hline KIOWA_-1200_34.FIN2 & 569 & 0.65 & 0.08678 & 0.000495 & 3.011 & 0.024 & 0.2498 & 0.0021 & 0.73195 & 1409 \\
\hline KIOWA_1200_73.FIN2C & 412.4 & 0.34 & 0.0872 & 0.00085 & 2.794 & 0.0235 & 0.232 & 0.00155 & 0.16756 & 1353 \\
\hline KIOWA_1200_21.FIN2 & 783 & 0.81 & 0.08752 & 0.00041 & 2.838 & 0.026 & 0.2357 & 0.00185 & 0.92638 & 1362 \\
\hline KIOWA_1200_52.FIN2 & 1343 & 0.29 & 0.08757 & 0.000325 & 3.044 & 0.0125 & 0.2496 & 0.001 & 0.62265 & 1418.2 \\
\hline KIOWA_1200_47.FIN2 & 891 & 0.88 & 0.08796 & 0.000455 & 3.062 & 0.0125 & 0.2495 & 0.0011 & 0.27835 & 1422.7 \\
\hline KIOWA_1200_70.FIN2C & 222.5 & 0.88 & 0.0882 & 0.00065 & 3.068 & 0.024 & 0.2516 & 0.00165 & 0.36052 & 1425 \\
\hline KIOWA_1200_116.FIN2 & 870 & 0.73 & 0.08826 & 0.000415 & 2.929 & 0.0155 & 0.2392 & 0.00135 & 0.57435 & 1388.4 \\
\hline KIOWA_1200_12.FIN2 & 61.7 & 1.39 & 0.0885 & 0.00095 & 2.982 & 0.028 & 0.2454 & 0.00165 & 0.21654 & 1401 \\
\hline KIOWA_1200_112.FIN2 & 405 & 0.76 & 0.0883 & 0.0005 & 3.113 & 0.017 & 0.254 & 0.0015 & 0.43933 & 1434.7 \\
\hline KIOWA_1200-84.FIN2C & 185.1 & 1.24 & 0.0887 & 0.00115 & 2.987 & 0.04 & 0.24 & 0.0025 & 0.23561 & 1410 \\
\hline KIOWA_1200_39.FIN2C & 203 & 0.41 & 0.0902 & 0.0012 & 2.149 & 0.0335 & 0.1756 & 0.00345 & 0.72285 & 1171 \\
\hline KIOWA_1200_97.FIN2 & 137.5 & 0.36 & 0.0918 & 0.0007 & 3.141 & 0.0225 & 0.2501 & 0.0014 & 0.2956 & 1444 \\
\hline KIOWA_1200_81.FIN2 & 521 & 0.44 & 0.0916 & 0.0006 & 2.595 & 0.023 & 0.2052 & 0.00205 & 0.78775 & 1298 \\
\hline KIOWA_1200_53.FIN2 & 277 & 0.45 & 0.0945 & 0.00075 & 3.329 & 0.0255 & 0.2549 & 0.00195 & 0.57031 & 1488 \\
\hline KIOWA_1200_33.FIN2 & 604 & 0.05 & 0.1 & 0.00055 & 3.979 & 0.0295 & 0.2867 & 0.0015 & 0.54992 & 1628 \\
\hline KIOWA_1200_42.FIN2 & 798 & 0.07 & 0.1008 & 0.0008 & 3.917 & 0.032 & 0.2807 & 0.0026 & 0.61138 & 1622 \\
\hline KIOWA_1200_63a.FIN2c & 511 & 0.12 & 0.1013 & 0.00075 & 4.299 & 0.045 & 0.3092 & 0.00285 & 0.74125 & 1692 \\
\hline KIOWA_1200_71.FIN2 & 464 & 0.62 & 0.10139 & 0.000475 & 4.277 & 0.0215 & 0.306 & 0.00155 & 0.54955 & 1688.7 \\
\hline KIOWA_1200_108.FIN2C & 199 & 0.95 & $\begin{array}{r}0.1015 \\
0.10178\end{array}$ & 0.00085 & 4.048 & 0.029 & 0.2858 & 0.00195 & 0.32801 & 1642 \\
\hline KIOWA_1200_124.FIN2 & 676 & 0.43 & 0.10178 & 0.000485 & 4.416 & 0.023 & 0.3124 & 0.0017 & 0.64759 & 1715.7 \\
\hline KIOWA_1200_78.FIN2 & $\begin{array}{r}255 \\
381\end{array}$ & 0.61 & $\begin{array}{r}0.1019 \\
0.10185\end{array}$ & $\begin{array}{r}0.00075 \\
0.00445\end{array}$ & $\begin{array}{l}4.076 \\
4.168\end{array}$ & 0.0335 & 0.2907 & 0.0022 & 0.55001 & 1649 \\
\hline $\begin{array}{l}\text { KIOWA-1200 } \\
\text { KIOWA } 1200 \text { - FIN } 2\end{array}$ & $\begin{array}{r}381 \\
3352\end{array}$ & 0.47 & $\begin{array}{l}0.10185 \\
0.1021\end{array}$ & $\begin{array}{l}0.000445 \\
0.0006\end{array}$ & $\begin{array}{l}4.168 \\
4.128\end{array}$ & 0.0185 & 0.2945 & $\begin{array}{l}0.00125 \\
0.00155\end{array}$ & $\begin{array}{l}0.41778 \\
0.51308\end{array}$ & 1669.2 \\
\hline KIOWA $1200 \quad 122$.FIN2c & $\begin{array}{l}103.3 \\
103.3\end{array}$ & 0.78 & 0.1021 & $\begin{array}{r}.0000 \\
0.001\end{array}$ & $\begin{array}{l}\begin{array}{l}4.128 \\
4.432\end{array} \\
\end{array}$ & $\begin{array}{l}0.0285 \\
0.0435\end{array}$ & $\begin{array}{l}0.2916 \\
0.317\end{array}$ & $\begin{array}{l}0.00155 \\
0.0026\end{array}$ & $\begin{array}{l}0.51308 \\
0.46717\end{array}$ & $\begin{array}{l}1760 \\
1719\end{array}$ \\
\hline KIOWĀ_1200_24.FIN2 & 621 & 0.09 & 0.10216 & 0.000375 & 4.271 & 0.018 & 0.3046 & 0.00125 & 0.53045 & 1687 \\
\hline KIOWA_1200_79.FIN2 & 147 & 0.77 & 0.1021 & 0.0006 & 4.301 & 0.029 & 0.3056 & 0.00175 & 0.58812 & 1693 \\
\hline KIOWA_1200_93.FIN2 & 268 & 0.44 & 0.1022 & 0.0007 & 4.259 & 0.0265 & 0.3048 & 0.0021 & 0.48976 & 1686 \\
\hline KIOWA_1200_87.FIN2 & 770 & 0.31 & 0.1023 & 0.000395 & 4.221 & 0.031 & 0.2996 & 0.0017 & 0.79563 & 1677 \\
\hline KIOWA_1200_118.FIN2 & 1384 & 0.08 & 0.1024 & 0.00055 & 4.21 & 0.0285 & 0.2962 & 0.0024 & 0.73412 & 1675 \\
\hline KIOWA_1200_131.FIN2 & 221 & 0.62 & 0.1024 & 0.0006 & 4.253 & 0.025 & 0.3004 & 0.0015 & 0.44412 & 1683.6 \\
\hline KIOWA_1200_80.FIN2 & 399 & 0.39 & 0.1027 & 0.00055 & 4.328 & 0.026 & 0.3059 & 0.0018 & 0.59284 & 1697.2 \\
\hline KIOWA_1200_129.FIN2 & 505 & 0.46 & 0.10249 & 0.000495 & 4.4 & 0.024 & 0.3096 & 0.00165 & 0.63349 & 1711 \\
\hline KIOWA_1200_7.FIN2 & 213.2 & 0.36 & 0.10276 & 0.000465 & 4.187 & 0.0225 & 0.2947 & 0.0012 & 0.45244 & 1672 \\
\hline KIOWA_1200_41.FIN2 & 218.1 & 0.74 & 0.1027 & 0.00065 & 3.922 & 0.0265 & 0.2734 & 0.0016 & 0.56135 & 1618 \\
\hline KIOWA_1200_45.FIN2 & 1067 & 0.05 & 0.10306 & 0.00037 & 4.34 & 0.015 & 0.304 & 0.00125 & 0.59072 & 1700.5 \\
\hline KIOWA_1200_64.FIN2 & 429 & 0.86 & 0.1033 & 0.00055 & 4.345 & 0.0 & 0.3 & 0.0021 & 0.66467 & 1701 \\
\hline KIOWA_1200_16.FIN2 & 214 & 0.85 & 0.1033 & 0.00055 & 3.98 & 0.02 & & 0.00 & 0.52173 & 1629.6 \\
\hline KIOWA-1200_36.F & 311 & 0.32 & 0.1034 & 0.00 & 4.13 & 0.0 & & & 0.7233 & 1661 \\
\hline KIOWA_1200_59 & 450 & 0.20 & 0.1034 & 0.00 & 3.802 & 0.0 & & & 0.37588 & 1590 \\
\hline KIOWA_1200_13 & 118 & 0.71 & 0.1032 & 0.00085 & & 0.04 & & 0.00285 & 812 & 1644 \\
\hline KIOWA_1200_9C & $\begin{array}{r}171.9 \\
347\end{array}$ & 0.59 & $\begin{array}{l}0.1039 \\
0.1038\end{array}$ & 0.0007 & $\begin{array}{l}4.29 \\
4.26\end{array}$ & 0.02 & & $\begin{array}{r}0.0019 \\
0.019\end{array}$ & 0.49573 & $\begin{array}{r}1693 \\
16856\end{array}$ \\
\hline KIOWA_1200 & $\begin{array}{l}347 \\
713\end{array}$ & 0.63 & $\begin{array}{l}0.1038 \\
0.1035\end{array}$ & & 4.2 & 0.022 & 0.29 & 0.00145 & 0.41533 & $\begin{array}{l}1685.6 \\
1588\end{array}$ \\
\hline $\begin{array}{l}\text { KIOWA_1200_-114.FIN2 } \\
\text { KIOWA 1200 109.FIN2 }\end{array}$ & $\begin{array}{l}713 \\
739\end{array}$ & $\begin{array}{l}0.41 \\
0.25\end{array}$ & $\begin{array}{r}0.1035 \\
0.10361\end{array}$ & $\begin{array}{l}0.00065 \\
0.00043\end{array}$ & $\begin{array}{l}3.78 \\
4.49 \\
-19\end{array}$ & $\begin{aligned} 0.04 \\
0.018\end{aligned}$ & $\begin{array}{c}0.2 \\
0.31\end{array}$ & $\begin{array}{l}0.0032 \\
0.0012\end{array}$ & $\begin{array}{l}0.87296 \\
0.45377\end{array}$ & $\begin{array}{r}1588 \\
1729.6\end{array}$ \\
\hline $\begin{array}{l}\text { KIOWA_1200-109.FIN2 } \\
\text { KIOWA } 1200 \text { 17.FIN }\end{array}$ & 1770 & $\begin{array}{l}0.25 \\
0.40\end{array}$ & $\begin{array}{r}0.10361 \\
0.1041\end{array}$ & $\begin{array}{r}0.00043 \\
0.0009\end{array}$ & $\begin{array}{l}4.42 \\
4.12\end{array}$ & $\begin{array}{l}0.0185 \\
0.0375\end{array}$ & $\begin{array}{l}0.3129 \\
0.2945\end{array}$ & $\begin{array}{r}0.0012 \\
0.00305\end{array}$ & 0.45377 & $\begin{array}{r}1729.6 \\
1658\end{array}$ \\
\hline KIOWA 1200 76.FIN2 & 221 & 0.71 & 0.1041 & 0.00055 & 4.35 & 0.024 & 0.3054 & 0.00145 & 0.35034 & 1702.1 \\
\hline KIOWA ${ }^{-1} 1200^{-14}$ 14FIN2 & 437 & 0.25 & 0.104 & 0.00055 & 4.06 & 0.0235 & 0.2846 & 0.00125 & 0.46016 & 1646.9 \\
\hline KIOWA-1200_5.FIN2 & 74.9 & 0.45 & 0.1044 & 0.001 & 4.195 & 0.0385 & 0.2892 & 0.00215 & 0.29163 & 1671 \\
\hline KIOWA_1200_18.FIN2 & 291 & 1.43 & 0.10412 & 0.00048 & 4.293 & 0.0205 & 0.301 & 0.00105 & 0.33861 & 1691 \\
\hline KIOWA_1200_94.FIN2 & 277 & 0.56 & 0.1044 & 0.0006 & 4.176 & 0.0245 & 0.29 & 0.00185 & 0.55882 & 1668.7 \\
\hline KIOWA_1200_10.FIN2 & 150.9 & 0.68 & 0.1042 & 0.000 & 3.96 & 0.0245 & 0.2781 & 0.0015 & 0.34558 & 1626 \\
\hline KIOWA_1200_22.FIN2 & 364 & 0.19 & 0.1045 & 0.00055 & 4.489 & 0.0295 & 0.313 & 0.0018 & 0.64515 & 1730 \\
\hline KIOWA_1200_58.FIN2 & 414 & 0.29 & 0.1049 & 0.00055 & 4.551 & 0.0295 & 0.3129 & 0.00175 & 0.6496 & 1738 \\
\hline KIOWA_1200_60.FIN2 & 276 & 0.36 & 0.1051 & 0.00055 & 4.478 & 0.0225 & 0.36 & 0.00155 & 0.38867 & 1725.7 \\
\hline A_1200_75.F & 219.8 & 0.46 & $0.1 c$ & 0.00 & & & & 0.0018 & 0.45076 & 1711 \\
\hline KIOWA_1200_9.FI & 167.8 & 0.81 & 0. & 0.00 & & & & 0.0 & 0.43664 & 1728 \\
\hline KIOWA_1200_65.FI & 778 & 0.36 & 0.10633 & 0.00 & & & & 0.00155 & 0.5549 & 1719.5 \\
\hline KIOWA_1200_130.F & 584.1 & 0.33 & 0.10 & & 4.08 & 0.042 & & & 0.30 & 1645 \\
\hline KIOWA_1200_56.F & 186.7 & 0.74 & 0.16 & & 4.8 & 0 & & 0.00315 & 0.65067 & 1792 \\
\hline KIOWA_1200_86.FIN2 & 267 & 1.21 & & & 4.2 & 0.05 & 0.2888 & 0.00295 & 0.85544 & 1675 \\
\hline KIOWA_1200_50.FIN2 & 444 & 0.80 & 0.1075 & 0.0006 & 4.782 & 0.0275 & 0.32 & 0.0015 & 0.4325 & 1781.2 \\
\hline KIOWA_1200_51.FIN2r & 624 & 0.13 & 0.1081 & 0.0022 & 3.98 & 0.07 & 0.2685 & 0.00405 & 0.20637 & 1628 \\
\hline
\end{tabular}

\begin{tabular}{|c|c|}
\hline 65 & \\
\hline 9 & $\begin{array}{r}986 \\
1177\end{array}$ \\
\hline 3.85 & 1191 \\
\hline 3.85 & 1109.5 \\
\hline & \\
\hline 4.95 & 1211 \\
\hline 6.5 & $\begin{array}{l}1135 \\
105\end{array}$ \\
\hline 9 & $\begin{array}{l}1053 \\
1211\end{array}$ \\
\hline 8 & $\begin{array}{l}1211 \\
1160\end{array}$ \\
\hline 6.5 & $\begin{array}{l}1166 \\
1321\end{array}$ \\
\hline $\begin{array}{r}4.7 \\
3.75\end{array}$ & $\begin{array}{l}1321 \\
1510\end{array}$ \\
\hline $\begin{array}{r}3.15 \\
9.5\end{array}$ & $\begin{array}{l}1570 \\
1264\end{array}$ \\
\hline 6 & 1439 \\
\hline & 1345 \\
\hline 7.5 & 1363 \\
\hline 3.1 & 1436 \\
\hline 3.1 & 1436 \\
\hline & 1446 \\
\hline $\begin{array}{r}4.05 \\
7\end{array}$ & $\begin{array}{l}1382 \\
1414\end{array}$ \\
\hline 4.25 & $\begin{array}{l}7414 \\
1460\end{array}$ \\
\hline & 1391 \\
\hline 11 & 1042 \\
\hline 5.5 & 1439 \\
\hline 6.5 & 1202 \\
\hline & 1465 \\
\hline 6 & 1625 \\
\hline 6.5 & 1593 \\
\hline 8.5 & 1736 \\
\hline 4.05 & 1720 \\
\hline 6 & $\begin{array}{l}1622 \\
1752\end{array}$ \\
\hline 4.4 & $\begin{array}{l}1752 \\
1742\end{array}$ \\
\hline 6.5 & 1648 \\
\hline $\begin{array}{l}3.7 \\
55\end{array}$ & $\begin{array}{l}1664 \\
1654\end{array}$ \\
\hline 5.5 & $\begin{array}{l}1651 \\
1774\end{array}$ \\
\hline 8.5 & $\begin{array}{l}1774 \\
1774\end{array}$ \\
\hline $\begin{array}{l}3.5 \\
5.5\end{array}$ & $\begin{array}{l}1714 \\
1718\end{array}$ \\
\hline 5.5 & $\begin{array}{l}1718 \\
1716\end{array}$ \\
\hline $\begin{array}{l}5 \\
6\end{array}$ & $\begin{array}{l}1716 \\
1691\end{array}$ \\
\hline $\begin{array}{r}6 \\
5.5\end{array}$ & $\begin{array}{l}1691 \\
1671\end{array}$ \\
\hline 5.5 & $\begin{array}{l}16 / 1 \\
1693\end{array}$ \\
\hline 4.8 & $\begin{array}{l}1693 \\
1719\end{array}$ \\
\hline $\begin{array}{l}4.9 \\
45\end{array}$ & \\
\hline 4.4 & 1666 \\
\hline 5.5 & 1557 \\
\hline 2.95 & 1711 \\
\hline & $\begin{array}{l}1714 \\
171\end{array}$ \\
\hline $\begin{array}{l}4.0 \\
7.5\end{array}$ & $\begin{array}{l}1616 \\
1631\end{array}$ \\
\hline 10 & 1525 \\
\hline 8.5 & 1612 \\
\hline 5.5 & 1714 \\
\hline 4.4 & 1687 \\
\hline 9.5 & \\
\hline 3.45 & 1755 \\
\hline 7.5 & \\
\hline 4.5 & 1718 \\
\hline 4.55 & \\
\hline 7.5 & \\
\hline 4 & \\
\hline 5 & $\begin{array}{r}1658 \\
1581\end{array}$ \\
\hline 5.5 & 1755 \\
\hline 5.5 & 1754 \\
\hline 4.15 & 1732 \\
\hline $\begin{array}{r}5.5 \\
5\end{array}$ & $\begin{array}{l}17 \\
17\end{array}$ \\
\hline $\begin{array}{r}5 \\
3.45\end{array}$ & $\begin{array}{l}1730 \\
1701\end{array}$ \\
\hline 7.5 & 1572 \\
\hline 10 & \\
\hline $\begin{array}{r}10.5 \\
4.9\end{array}$ & $\begin{array}{l}16 \\
17\end{array}$ \\
\hline & 153 \\
\hline
\end{tabular}


KIOWA_1200_98.FIN2 KIOWA-1200_2.FIN2 KIOWA-1200_411.FIN2 KIOWA_1200_30.FIN2 KIOWA-1200_26.FIN2C KIOWA-1200_20.FIN2 KIOWA 1200 - 6.FIN2 K1OWA-1200-31.FIN2 KIOWA_1200-107.FIN2

\begin{tabular}{rrrr}
464 & 0.30 & 0.10863 & 0.00047 \\
64.4 & 1.30 & 0.11 & 0.00115 \\
104.7 & 0.48 & 0.116 & 0.00125 \\
313 & 0.43 & 0.1131 & 0.00075 \\
264 & 0.17 & 0.1198 & 0.00075 \\
1170 & 0.09 & 0.1577 & 0.00105 \\
260.7 & 0.68 & 0.1615 & 0.00085 \\
530 & 0.14 & 0.1899 & 0.00155 \\
14.1 & 9.38 & 0.245 & 0.0055 \\
76 & 0.64 & 0.366 & 0.0085 \\
58.4 & 0.76 & 0.0601 & 0.0037 \\
144.3 & 1.42 & 0.0588 & 0.0022 \\
199 & 0.65 & 0.087 & 0.0019 \\
\hline
\end{tabular}

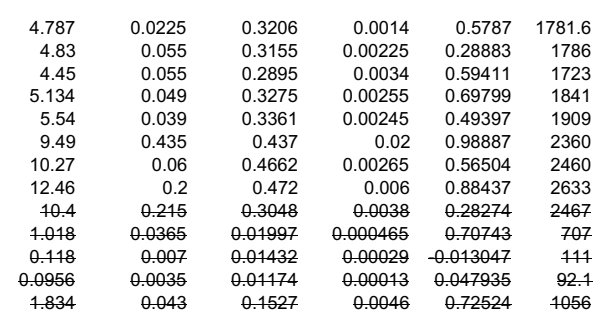

$\begin{array}{r}1781.6 \\ 1786 \\ 1723 \\ 1841 \\ 1909 \\ 2360 \\ 2460 \\ 2633 \\ 2467 \\ 707 \\ 111 \\ 92.1 \\ \hline\end{array}$

$\begin{array}{rr}3.9 & 1795 \\ 9 & 1770 \\ 9.5 & 1636 \\ 8.5 & 1825 \\ 6 & 1869 \\ 50 & 2300 \\ 5 & 2466 \\ 15.5 & 2489 \\ 18.5 & 1712 \\ 18.5 & 127.4 \\ 6 & 91.7 \\ 3.25 & 75.2\end{array}$

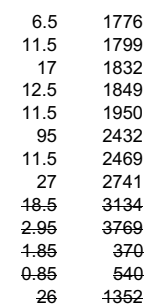

$\begin{array}{ll}7.5 & 1776 \\ 20 & 1799.0\end{array}$

$\begin{array}{rr}7.20 & 1779.0 \\ 20.5 & 1832.0 \\ 12 & 1849.0 \\ 11 & 1950\end{array}$

$\begin{array}{ll}12 & 1849.0 \\ 11 & 1950.0 \\ 11 & 2432.0\end{array}$

$\begin{array}{rr}11 & 2432.0 \\ 9 & 2469.0 \\ 13.5 & 2641.0\end{array}$

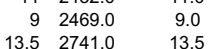

33.5 DISCARD DISCARD

115 DISCARD DISCARD

75 DISCARD DISCARD

Sample Name: Kiowa-1395
Analysis ID
KIOWA 1395 33.

KIOWAA-1395_33.FIN2
KIOWA_ 1395_56FIN2

KIOWA-1395_56.FIN2

KIOWA_1395_3.FIN2

KIOWA_1395_79.FIN2

KIOWA_1395_22.FIN2

KIOWA_1395_12.FIN2

KIOWA_1395_116.FIN2

KIOWA_1395_45.FIN2

KIOWA_1395_40.FIN2

KIOWA_1395_81.FIN2
KIOWA_1395_108.FIN2

KIOWA_1395_71.FIN2

KIOWA_1395_28.FIN2

KIOWA-1395-46.FIN2

KIOWA-1395_46.FIN2

KIOWA-1395_27.FIN2

KIOWA_1395-115.FIN2

KIOWA-1395_48.FIN2

KIOWA-1395-41.FIN2

KIOWA-1395_99.FIN2

KIOWA_1395_112.FIN2

KIOWA 1395_62.FIN2

KIOWA-1395-109.FIN2
KIOWA_1395_13.FIN2

KIOWA_1395_16.FIN2

KIOWA_1395_44.FIN2

KIOWA-1395_73.FIN2

KIOWA_1395_24.FIN2

KIOWA-1395-111.FIN2

KIOWA-1395_98.FIN2

KIOWA-1395-129.FIN2

KIOWA-1395-89.FIN2

KIOWA-1395_30.FIN2

KIOWA_1395_49.FIN2

KIOWA_1395_-107.FIN2

KIOWA_1395_107.FIN2
KIOWA 1395 91.FIN2

KIOWA-1395_55.FIN2

KIOWA 1395 90.FIN2

KIOWA_1395_105.FIN2

KIOWA_1395_96.FIN2

KIOWA_1395_57.FIN2

KIOWA_1395_60.FIN2

KIOWA_1395_54.FIN2
KIOWA_1395_14.FIN2

KIOWA_1395_101.FIN2

KIOWA_1395_-1.FIN2

KIOWA_1395_80.FIN2

\begin{tabular}{|c|c|c|c|c|c|c|c|c|c|}
\hline 136.7 & 0.31 & 0.0495 & 0.00175 & 0.1098 & 0.0037 & 0.01614 & 0.00017 & -0.076399 & 10 \\
\hline $\begin{array}{r}116.6 \\
869\end{array}$ & $\begin{array}{l}0.92 \\
1.34\end{array}$ & $\begin{array}{l}0.0485 \\
0.0537\end{array}$ & $\begin{array}{l}0.0012 \\
0.0007\end{array}$ & $\begin{array}{l}0.1634 \\
0.2079\end{array}$ & $\begin{array}{l}0.0042 \\
0.0026\end{array}$ & $\begin{array}{l}0.02429 \\
0.02821\end{array}$ & $\begin{array}{l}0.000235 \\
0.000195\end{array}$ & $\begin{array}{l}0.26517 \\
0.25089\end{array}$ & \\
\hline $\begin{array}{r}869 \\
264.1\end{array}$ & $\begin{array}{l}1.34 \\
1.23\end{array}$ & 0.0493 & 0.001 & $\begin{array}{l}0.2079 \\
0.1918\end{array}$ & 0.0026 & $\begin{array}{l}0.02821 \\
0.02823\end{array}$ & $\begin{array}{l}0.000195 \\
0.00029\end{array}$ & 0.25089 & \\
\hline 91 & 0.94 & 0.0509 & 0.00155 & 0.216 & 0.006 & 0.03085 & 0.000275 & $\begin{array}{r}0.27301 \\
0.028739\end{array}$ & \\
\hline 523 & 2.80 & 0.0515 & 0.0006 & 0.3302 & 0.0039 & 0.04668 & 0.00029 & 0.30771 & \\
\hline 355 & 0.32 & 0.0549 & 0.0006 & 0.4537 & 0.0049 & 0.06067 & 0.000335 & 0.26221 & \\
\hline 186.2 & 0.41 & 0.0558 & 0.0008 & 0.587 & 0.0085 & 0.07669 & 0.000495 & 0.13286 & \\
\hline 220.6 & 0.99 & 0.0577 & 0.00055 & 0.631 & 0.0055 & 0.08049 & 0.00047 & 0.2232 & \\
\hline 180.4 & 0.40 & 0.0599 & 0.0007 & 0.804 & 0.008 & 0.0985 & 0.00065 & 0.052099 & \\
\hline & & & & & & & & & \\
\hline 75.1 & 0.40 & 0.0707 & 0.0009 & $\begin{array}{l}1.492 \\
1.02\end{array}$ & 0.018 & 0.1546 & 0.00105 & 0.24754 & \\
\hline 263.5 & 0.46 & 0.0712 & 0.0005 & 1.624 & 0.012 & 0.1643 & 0.00085 & 0.37321 & \\
\hline 281 & 0.36 & 0.073 & 0.0005 & 1.785 & 0.014 & 0.176 & 0.001 & 0.3633 & \\
\hline 285.7 & 0.24 & 0.0736 & 0.00055 & 1.79 & 0.012 & 0.175 & 0.00135 & 0.40399 & \\
\hline 94 & 0.62 & 0.0749 & 0.00075 & 1.905 & 0.0195 & 0.1837 & 0.0012 & 0.34297 & \\
\hline 108.2 & 0.88 & 0.0739 & 0.00065 & 1.906 & 0.018 & 0.1854 & $\begin{array}{r}0.001 \\
0\end{array}$ & 0.29026 & \\
\hline 137 & 0.94 & $\begin{array}{r}0.075 \\
\end{array}$ & 0.00065 & 1.904 & 0.016 & 0.1847 & $\begin{array}{r}0.0011 \\
0.0009\end{array}$ & 0.29061 & \\
\hline 197.5 & 0.56 & 0.07485 & 0.000475 & 1.896 & 0.013 & 0.1827 & 0.00095 & $\begin{array}{r}0.48282 \\
\end{array}$ & \\
\hline 132.3 & 1.09 & 0.0755 & 0.0007 & 1.86 & 0.016 & 0.1802 & 0.00075 & 0.047053 & \\
\hline 90.1 & 1.00 & 0.0751 & 0.00075 & 1.934 & 0.021 & 0.1866 & 0.0011 & $\begin{array}{r}0.36091 \\
\end{array}$ & \\
\hline $\begin{array}{l}93.7 \\
270\end{array}$ & 0.71 & 0.0758 & 0.00095 & 1.846 & $\begin{array}{r}0.022 \\
0145\end{array}$ & 0.1771 & $\begin{array}{r}0.0012 \\
0.0125\end{array}$ & $\begin{array}{r}0.097219 \\
\end{array}$ & \\
\hline 270 & 0.65 & $\begin{array}{r}0.0751 \\
0.0751\end{array}$ & 0.00055 & 1.896 & 0.0145 & 0.1815 & 0.00125 & $\begin{array}{l}0.45599 \\
0\end{array}$ & \\
\hline 240 & 33 & 0.07516 & 0.00046 & 1.954 & $\begin{array}{r}0.011 \\
0.0155\end{array}$ & $\begin{array}{r}0.19 \\
0\end{array}$ & $\begin{array}{r}0.0007 \\
0\end{array}$ & 27349 & \\
\hline 129.6 & & $\begin{array}{l}0.0751 \\
0.074\end{array}$ & 0.000 & $\begin{array}{l}1.889 \\
1814\end{array}$ & & & 0.00095 & & \\
\hline 288 & & & & & & & & & \\
\hline 108.7 & & & & & & & & & \\
\hline & & $\begin{array}{l}0.0753 \\
0.0754\end{array}$ & & & & & & & \\
\hline $\begin{array}{l}91.8 \\
199\end{array}$ & & $\begin{array}{l}0.0754 \\
0.0754\end{array}$ & & $\begin{array}{l}1.919 \\
1.934\end{array}$ & & & & $\begin{array}{l}0.15877 \\
0.26703\end{array}$ & \\
\hline $\begin{array}{r}199 \\
1999\end{array}$ & $\begin{array}{l}0.55 \\
0.56\end{array}$ & $\begin{array}{l}0.0754 \\
0.0754\end{array}$ & & $\begin{array}{l}1.934 \\
1.919\end{array}$ & & & $\begin{array}{r}0.001 \\
0.00085\end{array}$ & $\begin{array}{l}0.26703 \\
0.21509\end{array}$ & \\
\hline $\begin{array}{l}199.9 \\
132.4\end{array}$ & $\begin{array}{l}0.56 \\
0.79\end{array}$ & $\begin{array}{r}0.0754 \\
0.076\end{array}$ & $\begin{array}{r}0.0006 \\
0.00115\end{array}$ & $\begin{array}{l}1.919 \\
1.789\end{array}$ & $\begin{array}{r}0.015 \\
0.0285\end{array}$ & $\begin{array}{l}0.1837 \\
0.1722\end{array}$ & $\begin{array}{l}0.000055 \\
0.0015\end{array}$ & $\begin{array}{l}0.21509 \\
0.39023\end{array}$ & \\
\hline $\begin{array}{l}132.4 \\
115.8\end{array}$ & 0.61 & 0.0757 & 0.00085 & 1.733 & & & $\begin{array}{l}0.0015 \\
0.0014\end{array}$ & $\begin{array}{l}0.39023 \\
0.32667\end{array}$ & \\
\hline 140.5 & 1.00 & 0.0752 & & 1.89 & & & 0.00095 & $\begin{array}{l}0.32061 \\
0.199\end{array}$ & \\
\hline 157.8 & 0.67 & 0.076 & & 1.90 & & & 0.00085 & -0.088539 & \\
\hline & 0.58 & 0.0759 & & 1.885 & 0.0165 & & 0.00105 & 0.38991 & \\
\hline 70.6 & & 0.0758 & & 1.809 & 0.025 & & 0.0013 & 0.38187 & \\
\hline 76.4 & & 0.0762 & & 1.897 & 0.02 & 0.181 & 0.00095 & 15744 & \\
\hline 137.2 & & 0.0758 & & 1.60 & & 0.15 & 0.00095 & 0.17769 & \\
\hline 80.7 & & 0.0776 & & 1.67 & & 0.1616 & 0.0018 & 0.019991 & \\
\hline 242 & & 0.0757 & & 1.87 & & 0.179 & 0.00105 & 0.36685 & \\
\hline 152.6 & & 0.0757 & & 1.86 & 0.0 & 0.18 & 0.00095 & 0.19218 & \\
\hline 273.4 & & 0.07 & & 1.84 & 0.01 & & 0.0016 & 0.66486 & \\
\hline 160.7 & & & & 1.5 & & & & & \\
\hline 102.3 & & & & 1. & & & & & \\
\hline 122.5 & & & & 1.9 & & & & & \\
\hline & & & & & & & & & \\
\hline 184 & & & & & & & & & \\
\hline $\begin{array}{r}72.4 \\
7096\end{array}$ & & & & & & & & & \\
\hline $\begin{array}{l}209.6 \\
38.8\end{array}$ & & & & & & & & & \\
\hline & & & & & & & & & \\
\hline & & & & & & & & & \\
\hline & & & & & & & & & \\
\hline $\begin{array}{l}122.9 \\
73.8\end{array}$ & $\begin{array}{l}1.16 \\
1.03\end{array}$ & & $\begin{array}{r}0.000 \\
0.00\end{array}$ & & & & & & \\
\hline $\begin{array}{c}73.8 \\
153\end{array}$ & $\begin{array}{l}1.03 \\
0.85\end{array}$ & $\begin{array}{r}0.076 \\
0.0764\end{array}$ & $\begin{array}{r}0.0007 \\
0.00065\end{array}$ & & & & $\begin{array}{l}0.0011 \\
0.0009\end{array}$ & $\begin{array}{r}0.19533 \\
0.078095\end{array}$ & \\
\hline $\begin{array}{c}153 \\
96.9\end{array}$ & $\begin{array}{l}0.85 \\
0.85\end{array}$ & $\begin{array}{l}0.0764 \\
0.076\end{array}$ & $\begin{array}{r}0.00065 \\
0.0011\end{array}$ & & & & & $\begin{array}{l}0.078095 \\
0.4525\end{array}$ & \\
\hline $\begin{array}{r}96.9 \\
196.7\end{array}$ & $\begin{array}{l}0.85 \\
1.36\end{array}$ & $\begin{array}{r}0.076 \\
0.0766\end{array}$ & $\begin{array}{r}0.0011 \\
0.00065\end{array}$ & $\begin{array}{l}1.72 \\
1.71\end{array}$ & $\begin{array}{l}0.02 \\
0.01\end{array}$ & $\begin{array}{r}0.1672 \\
0.161\end{array}$ & $\begin{array}{l}0.00245 \\
0.00085\end{array}$ & $\begin{array}{r}0.4524 \\
0.30336\end{array}$ & \\
\hline $\begin{array}{l}196.7 \\
124.6\end{array}$ & $\begin{array}{l}1.36 \\
1.35\end{array}$ & $\begin{array}{l}0.0766 \\
0.0767\end{array}$ & $\begin{array}{r}0.000655 \\
0.0008\end{array}$ & $\begin{array}{l}1.1711 \\
1.967\end{array}$ & & $\begin{array}{r}0.161 \\
0.1864\end{array}$ & $\begin{array}{r}0.000055 \\
0.0013\end{array}$ & 30336 & \\
\hline $\begin{array}{r}124.6 \\
62\end{array}$ & $\begin{array}{l}1.35 \\
0.31\end{array}$ & $\begin{array}{l}0.0767 \\
0.0769\end{array}$ & $\begin{array}{r}0.0008 \\
0.00105\end{array}$ & $\begin{array}{l}1.837 \\
1.837\end{array}$ & $\begin{array}{r}0.019 \\
0.0235\end{array}$ & $\begin{array}{l}0.1864 \\
0.1724\end{array}$ & $\begin{array}{r}0.00133 \\
0.00155\end{array}$ & $\begin{array}{l}0.341111 \\
0.24895\end{array}$ & \\
\hline
\end{tabular}

$\begin{array}{rrrr}\text { rror } & \text { Age (Ma) } & \text { 10 error } & \text { Age (Ma) } \\ 3.3 & 103.2 & 1.05 & 140 \\ 3.65 & 154.7 & 1.5 & 117 \\ 2.15 & 179.3 & 1.2 & 342 \\ 3.3 & 179.4 & 1.35 & 180 \\ 5 & 195.8 & 1.7 & 200 \\ 3 & 294.1 & 1.8 & 252 \\ 3.45 & 379.6 & 2.05 & 393 \\ 5 & 476.2 & 2.95 & 424 \\ 3.5 & 499 & 2.8 & 517 \\ 4.55 & 605.5 & 3.8 & 594 \\ 4 & 643.9 & 3 & 617 \\ 7.5 & 928 & 6 & 948 \\ 4.55 & 980.2 & 4.8 & 972 \\ 5 & 1045 & 5.5 & 1011 \\ 4.5 & 1039 & 7.5 & 1030 \\ 6.5 & 1087 & 6.5 & 1052 \\ 6 & 1097 & 5.5 & 1054 \\ 5.5 & 1094 & 6 & 1060 \\ 4.6 & 1081 & 5 & 1063 \\ 5.5 & 1067.8 & 4.05 & 1067 \\ 7.5 & 1103 & 6 & 1068 \\ 8 & 1051 & 6.5 & 1069 \\ 5 & 1074 & 6.5 & 1070 \\ 3.9 & 1121.4 & 3.9 & 1071 \\ 5.5 & 1063 & 5 & 1071 \\ 6 & 1054 & 6 & 1072 \\ 6.5 & 1073 & 6.5 & 1073 \\ 5 & 1020 & 7 & 1075 \\ 6 & 1086 & 5.5 & 1076 \\ 4.9 & 1105 & 5.5 & 1078 \\ 5 & 1086.7 & 4.55 & 1079 \\ 10 & 1024 & 8.5 & 1079 \\ 6.5 & 998 & 8 & 1079 \\ 5 & 1084 & 5.5 & 1082 \\ 5.5 & 1083.2 & 4.5 & 1085 \\ 5.5 & 1068 & 5.5 & 1085 \\ 8.5 & 1030 & 7 & 1087 \\ 7 & 1072 & 5.5 & 1087 \\ 6 & 925 & 5 & 1088 \\ 9.5 & 964 & 10 & 1089 \\ 4.6 & 1061 & 5.5 & 1089 \\ 4.95 & 1072 & 5 & 1090 \\ 5.5 & 1055 & 8.5 & 1093 \\ 6 & 1095 & 6 & 1093 \\ 6.5 & 1085 & 5 & 1094 \\ 5 & 1077 & 6 & 1095 \\ 7.5 & 1023 & 7.5 & 1097 \\ 4.95 & 1064.8 & 4.1 & 1097 \\ 8 & 1082 & 7.5 & 1097 \\ 5.5 & 1122 & 5.5 & 1097 \\ 12 & 1098 & 10 & 1099 \\ 7 & 1104.8 & 4.75 & 1100 \\ 5 & 1049 & 6.5 & 1100 \\ 6.5 & 1065 & 6 & 1100 \\ 7 & 1112 & 6 & 1101 \\ 5 & 1074 & 5 & 1102 \\ 9.5 & 1000 & 14 & 1103 \\ 5.5 & 962 & 4.75 & 1104 \\ 6.5 & 1101 & 7 & 1104 \\ 8.5 & 1027 & 8.5 & 1105 \\ & & & \end{array}$

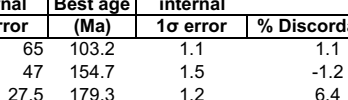

$\begin{array}{rr}40 & 179.4 \\ 55 & 195.8\end{array}$

$\begin{array}{ll}55 & 195.8 \\ 24 & 294.1 \\ 24 & 379.6 \\ 3 & 476.2\end{array}$

$\begin{array}{ll}24 & 379.6 \\ 30 & 476.2\end{array}$

$\begin{array}{rr}20 & 499.0 \\ 25.5 & 605.5\end{array}$

$\begin{array}{rr}19 & 643.9\end{array}$

$\begin{array}{ll}26 & 948.0 \\ 15 & 972.0\end{array}$

$\begin{array}{rr}15 & 1011.0 \\ 155 & 1030\end{array}$

$\begin{array}{rr}20.5 & 1052.0 \\ 19 & 1054.0\end{array}$

$\begin{array}{ll}19 & 1054.0 \\ 18 & 1060.0\end{array}$

$\begin{array}{rrr}12.5 & 1063.0\end{array}$

$\begin{array}{rr}18.5 & 1067.0 \\ 19.5 & 1068.0 \\ 23.5 & 1069.0\end{array}$

$\begin{array}{rr}23.5 & 1069.0 \\ 15 & 1070.0\end{array}$

$\begin{array}{rr}17.5 & 1071.0 \\ 14.5 & 1072.0\end{array}$

$\begin{array}{ll}20 & 1073.0 \\ 16 & 1075.0\end{array}$

$\begin{array}{ll}16 & 1075.0 \\ 19 & 1076.0\end{array}$

$\begin{array}{ll}16 & 1078.0\end{array}$

$\begin{array}{rr}16.5 & 1079.0 \\ 31 & 1079.0\end{array}$

$\begin{array}{rr}22.5 & 1079.0 \\ 17 & 1082.0\end{array}$

$\begin{array}{ll}17 & 1082.0 \\ 16 & 1085.0\end{array}$

$\begin{array}{rr}17 & 1085.0 \\ 24.5 & 1087.0\end{array}$

$\begin{array}{ll}22.5 & 1087.0 \\ 19.5 & 1088.0\end{array}$

$\begin{array}{rr}33 & 1089.0 \\ 145 & 10890\end{array}$

14.51089 .0

$\begin{array}{rr}17.5 & 1093.0 \\ 17.5 & 1093.0\end{array}$

$\begin{array}{rr}17.5 & 1093.0 \\ 22 & 1094.0\end{array}$

$\begin{array}{rr}17 & 1095.0 \\ 21.5 & 1097.0 \\ 16 & 1097.0\end{array}$

$\begin{array}{rr}21.5 & 1097.0 \\ 16 & 1097.0 \\ 24.5 & 1097.0\end{array}$

$\begin{array}{rr}24.5 & 1097.0 \\ 13.5 & 1097.0\end{array}$

$\begin{array}{rr}37 & 1099.0 \\ 21.5 & 1100.0\end{array}$

$\begin{array}{ll}13.5 & 1100.0 \\ 17.5 & 1100 \\ 20 & 1101.0\end{array}$

$\begin{array}{rr}20 & 1101.0 \\ 17 & 1102.0\end{array}$

$\begin{array}{ll}28.5 & 1103.0 \\ 16.5 & 1104.0 \\ 2.5 & 1104.0\end{array}$

$\begin{array}{rr}20.5 & 1104 \\ 27 & 1105.0\end{array}$

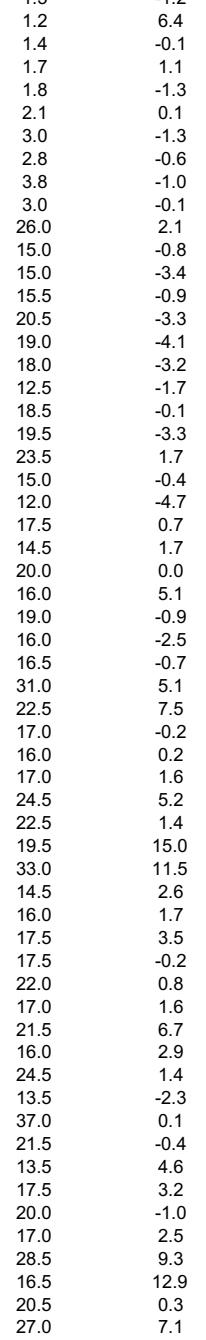




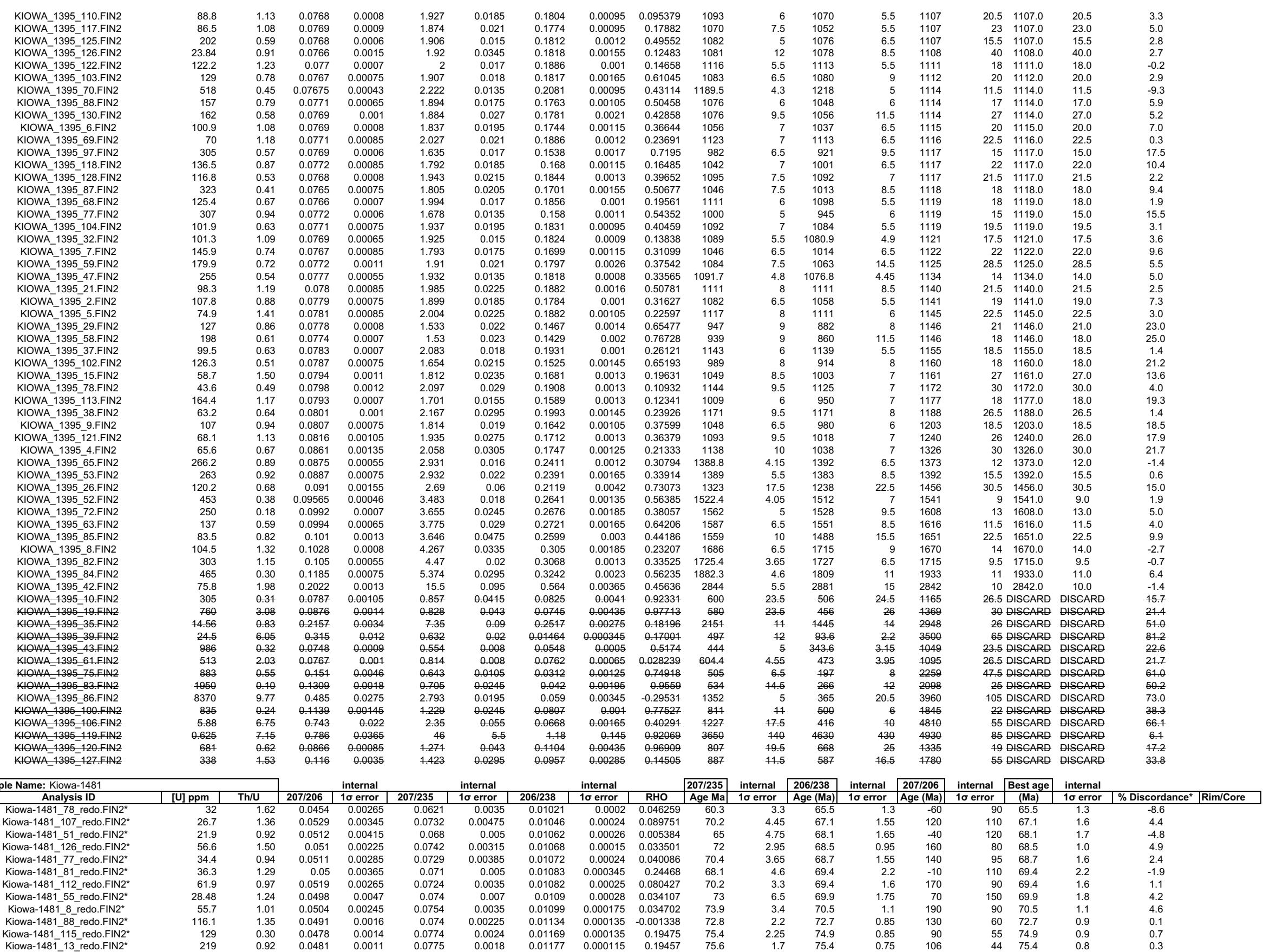




\begin{tabular}{|c|c|c|c|c|c|c|c|c|c|c|}
\hline Kiowa_1481_137.FIN2r & 61.8 & 0.83 & 0.056 & 0.007 & 0.087 & 0.01 & 0.01202 & 0.00048 & -0.023048 & 84 \\
\hline Kiowa_1481_12.FIN2 & 270 & 0.59 & 0.0501 & 0.0009 & 0.1007 & 0.0018 & 0.01416 & 0.0001 & 0.1374 & 97.2 \\
\hline Kiowa_1481_114.FIN2 & 120.6 & 0.25 & 0.0486 & 0.0011 & 0.1117 & 0.0024 & 0.01678 & 0.00014 & 0.12547 & 107.2 \\
\hline Kiowa_1481_48.FIN2 & 175 & 0.51 & 0.0539 & 0.00135 & 0.1855 & 0.00465 & 0.02498 & 0.00023 & 0.20308 & 171.7 \\
\hline Kiowa_1481_121.FIN2 & 414 & 1.13 & 0.0498 & 0.00065 & 0.1807 & 0.00255 & 0.02607 & 0.000265 & 0.47565 & 168.3 \\
\hline Kiowa_1481_6.FIN2 & 144 & 1.28 & 0.0499 & 0.00085 & 0.19 & 0.00315 & 0.02723 & 0.000205 & 0.15125 & 176.1 \\
\hline & 228 & 0.27 & 0.0515 & 0.0009 & 0.2155 & 0.004 & 0.02959 & 0.000295 & 0.3817 & 197.3 \\
\hline $\begin{array}{l}\text { Kiowa_1481_84.FIN2 } \\
\text { Kiowa 1481 64 FIN }\end{array}$ & $\begin{array}{l}87.9 \\
325\end{array}$ & 0.44 & 0.0567 & 0.00135 & 0.253 & 0.006 & 0.03208 & 0.00024 & 0.036268 & 228.2 \\
\hline $\begin{array}{l}\text { Kiowa_- 1481-64.FIN2 } \\
\text { Kiowa } 1481 \text { 26.FIN2 }\end{array}$ & $\begin{array}{l}325 \\
213\end{array}$ & $\begin{array}{l}0.98 \\
0.92\end{array}$ & $\begin{array}{l}0.0504 \\
0.0514\end{array}$ & $\begin{array}{l}0.00065 \\
0.00085\end{array}$ & $\begin{array}{l}0.2273 \\
0.2618\end{array}$ & $\begin{array}{l}0.00285 \\
0.00395\end{array}$ & $\begin{array}{l}0.03243 \\
0.03619\end{array}$ & $\begin{array}{l}0.000225 \\
0.000325\end{array}$ & $\begin{array}{l}0.15909 \\
0.2716\end{array}$ & $\begin{array}{r}207.6 \\
236.2\end{array}$ \\
\hline Kiowa_1481_20.FIN2 & 287 & 1.12 & $\begin{array}{l}0.0523 \\
0.0523\end{array}$ & 0.00055 & 0.326 & $\begin{array}{l}0.0033 \\
0.0035\end{array}$ & 0.04359 & 0.000235 & $\begin{array}{l}0.22428 \\
0.21710\end{array}$ & $\begin{array}{r}386.2 \\
286\end{array}$ \\
\hline Kiowa_1481_136.FIN2 & 283 & 0.44 & 0.05541 & 0.000495 & 0.5164 & 0.0047 & 0.06749 & 0.000375 & 0.3729 & 422.8 \\
\hline Kiowa_1481_19.FIN2 & 511 & 0.22 & 0.0569 & 0.0005 & 0.572 & 0.006 & 0.0706 & 0.0007 & 0.60832 & 459 \\
\hline Kiowa_1481_17.FIN2 & 343 & 0.38 & 0.0591 & 0.0006 & 0.629 & 0.0065 & 0.0748 & 0.00065 & 0.45638 & 494 \\
\hline Kiowa_1481_16.FIN2 & 171.1 & 0.41 & 0.058 & 0.00065 & 0.631 & 0.0075 & 0.0759 & 0.0006 & 0.43186 & 496.1 \\
\hline Kiowa_1481_34.FIN2 & 529 & 0.24 & 0.059 & 0.0006 & 0.624 & 0.009 & 0.076 & 0.0011 & 0.74447 & 490 \\
\hline Kiowa_1481_38.FIN2 & 160.6 & 0.49 & 0.0581 & 0.00065 & 0.615 & 0.0065 & 0.0764 & 0.0005 & 0.31209 & 486.3 \\
\hline Kiowa_1481_54.FIN2 & 84 & 0.36 & 0.0582 & 0.00095 & 0.621 & 0.01 & 0.0768 & 0.0006 & 0.27192 & 488 \\
\hline Kiowa_1481_123.FIN2 & 148.1 & 0.32 & 0.058 & 0.0006 & 0.625 & 0.007 & 0.07735 & 0.00044 & 0.32453 & 491.5 \\
\hline Kiowa_1481_103.FIN2 & 94.2 & 0.70 & 0.059 & 0.00075 & 0.622 & 0.0085 & 0.0782 & 0.00055 & 0.34965 & 489 \\
\hline Kiowa_1481_95.FIN2 & 444 & 0.33 & 0.05754 & 0.00041 & 0.628 & 0.0075 & 0.0798 & 0.0009 & 0.79646 & 493.1 \\
\hline Kiowa_1481_87.FIN2 & 110.5 & 0.62 & 0.0574 & 0.0007 & 0.636 & 0.0075 & 0.0801 & 0.00055 & 0.28994 & 498.2 \\
\hline Kiowa_1481_110.FIN2 & 152.3 & 0.65 & 0.0567 & 0.0006 & 0.619 & 0.007 & 0.08037 & 0.000415 & 0.31653 & 488.1 \\
\hline Kiowa_1481_1.FIN2 & 400 & 0.46 & 0.05763 & 0.00035 & 0.6444 & 0.00415 & 0.08166 & 0.000355 & 0.46866 & 504.5 \\
\hline Kiowa_1481_73.FIN2 & 112.1 & 0.72 & 0.0578 & 0.0006 & 0.665 & 0.008 & 0.0829 & 0.00055 & 0.42344 & 516 \\
\hline Kiowa_1481_125.FIN2 & 198 & 0.39 & 0.0559 & 0.00055 & 0.646 & 0.007 & 0.0835 & 0.0007 & 0.4659 & 504.6 \\
\hline Kiowa_1481_111.FIN2 & 97.9 & 0.67 & 0.0569 & 0.0007 & 0.648 & 0.008 & 0.0838 & 0.0006 & 0.36808 & 508 \\
\hline Kiowa_1481_127.FIN2 & 396 & 0.77 & 0.0609 & 0.0006 & 0.81 & 0.0085 & 0.0961 & 0.00085 & 0.54543 & 601.2 \\
\hline Kiowa_1481_70.FIN2 & 414 & 0.87 & 0.0645 & 0.0007 & 0.973 & 0.015 & 0.1079 & 0.0009 & 0.57846 & 686 \\
\hline Kiowa_1481_93.FIN2 & 77.8 & 0.78 & 0.0777 & 0.00145 & 1.28 & 0.025 & 0.1203 & 0.00175 & 0.45194 & 833 \\
\hline Kiowa_1481_138.FIN2 & 177 & 0.31 & 0.0723 & 0.0007 & 1.24 & 0.0145 & 0.1237 & 0.001 & 0.51666 & 817 \\
\hline Kiowa_1481_128.FIN2 & 63.8 & 0.51 & 0.0703 & $\begin{array}{r}0.0008 \\
0\end{array}$ & 1.636 & 0.021 & 0.1686 & $\begin{array}{r}0.0017 \\
0.0105\end{array}$ & 0.51871 & 981 \\
\hline $\begin{array}{l}\text { Kowa_1481_ }{ }^{118 . F I N 2} \\
\text { Kolowa } 1481 \text { 135FEN2 }\end{array}$ & $\begin{array}{r}78.3 \\
58\end{array}$ & $\begin{array}{l}0.96 \\
0.63\end{array}$ & $\begin{array}{l}0.0718 \\
0.073\end{array}$ & $\begin{array}{l}0.00085 \\
0.00075\end{array}$ & $\begin{array}{l}1.437 \\
1708\end{array}$ & 0.017 & $\begin{array}{r}0.144 \\
0.162\end{array}$ & 0.00105 & $\begin{array}{r}0.3191 \\
0.35297\end{array}$ & 902 \\
\hline $\begin{array}{l}\text { Kiowa_ } 1481 \text { 1 } 135 \text {. IN } 2 \\
\text { Kiowa } 1481 \text { 130.FIN2 }\end{array}$ & $\begin{array}{r}58 \\
51.8\end{array}$ & $\begin{array}{l}0.63 \\
0.75\end{array}$ & $\begin{array}{l}0.0737 \\
0.0743\end{array}$ & $\begin{array}{l}0.00075 \\
0.0007\end{array}$ & $\begin{array}{l}1.708 \\
1717\end{array}$ & $\begin{array}{l}0.018 \\
0.017\end{array}$ & $\begin{array}{l}0.1682 \\
0.1664\end{array}$ & $\begin{array}{l}0.00115 \\
0.0009\end{array}$ & $\begin{array}{l}0.35297 \\
0.2482\end{array}$ & $\begin{array}{l}1012 \\
1012\end{array}$ \\
\hline Kiowa_1481_53.FIN2 & 216.6 & 0.73 & 0.07453 & $\begin{array}{r}0.000445 \\
\end{array}$ & 1.673 & 0.0095 & $\begin{array}{l}0.1664 \\
0.1619\end{array}$ & 0.00075 & 0.36472 & $\begin{array}{l}1012 \\
999.1\end{array}$ \\
\hline Kiowa_1481_3.FIN2 & 39.2 & 0.82 & 0.0754 & 0.00095 & 2.01 & 0.023 & 0.1934 & 0.0014 & 0.26836 & 1114 \\
\hline Kiowa_1481_134.FIN2 & 70.9 & 1.02 & 0.0755 & 0.00075 & 1.748 & 0.0215 & 0.1666 & 0.00115 & 0.37085 & 1019 \\
\hline Kiowa_1481_109.FIN2 & 30.7 & 1.03 & 0.076 & 0.00105 & 1.669 & 0.022 & 0.1633 & 0.00145 & 0.2929 & 993 \\
\hline Kiowa_1481_113.FIN2 & 103.7 & 1.22 & 0.0753 & 0.0006 & 1.831 & 0.0155 & 0.1771 & 0.0011 & 0.41264 & 1054 \\
\hline Kiowa_1481_85.FIN2 & 254 & 0.71 & 0.07515 & 0.000475 & 1.876 & 0.013 & 0.1792 & 0.001 & 0.47114 & 1071 \\
\hline Kiowa_1481_89.FIN2 & 160 & 0.77 & 0.0752 & 0.0006 & 1.836 & 0.018 & 0.1758 & 0.00135 & 0.61523 & 1057 \\
\hline Kiowa_1481_63.FIN2 & 377 & 0.59 & 0.0759 & 0.0006 & 1.871 & 0.0195 & 0.1778 & 0.00175 & 0.67931 & 1069 \\
\hline Kiowa_1481_15.FIN2 & 173 & 0.67 & 0.0755 & 0.00055 & 1.859 & 0.0155 & 0.1724 & 0.0011 & 0.51931 & 1066 \\
\hline Kiowa_1481_98.FIN2 & 208 & 0.60 & 0.0757 & 0.0005 & 1.807 & 0.014 & & 0.001 & 0.51809 & 1046 \\
\hline Kiowa_1481_94.FIN2 & 123 & 1.09 & 0.0763 & 0.00065 & 1.779 & 0.0 & & & & 1035 \\
\hline Kiowa_1481_2.FIN2 & 93 & 1.15 & 0.0 & 0.0008 & & 0.02 & 0.1693 & & 1592 & 1036 \\
\hline Kiowa_1481_46.FI & 182.7 & 1.07 & 0.07 & 0.00 & 1.805 & & & & 0.55361 & 1048.2 \\
\hline Kiowa_1481_75.FIN2 & 154 & 0.75 & & 0.00 & 1. & & & & & 1028 \\
\hline Kiowa_1481_11.FII & 32.2 & 1.43 & & 0.00 & & 0.0 & 0.1572 & 0.0013 & 0.27936 & 1014 \\
\hline Kiowa_1481_108.FI & 211 & 0.19 & 0.0776 & 0.00 & 1.768 & & & 024 & 7174 & 1030 \\
\hline Kiowa_148 & 29.1 & 0.88 & 0.0786 & 0.0011 & 1.81 & 0.02 & & 0.0017 & 4709 & 1044 \\
\hline Kiowa_1481_52.FIN2 & 79.6 & 0.71 & 0.079 & 0.00085 & 1.76 & 0.0 & 0.162 & 0.0015 & 6482 & 1027 \\
\hline Kiowa_1481_33.FIN2 & 190 & 0.75 & 0.0789 & 0.0007 & 1.716 & 0.0 & 0.15 & 0.0018 & 6387 & 1011 \\
\hline Kiowa_1481_41.FIN2 & 64.2 & 1.18 & 0.0806 & 0.0012 & 1.689 & 0.026 & 0.1522 & 0.002 & 0.41971 & 97 \\
\hline Kiowa_1481_80.FII & 344 & 1.16 & 0.0798 & $\begin{array}{r}0.0005 \\
0.00655\end{array}$ & 2.22 & 0.0185 & 0.2006 & 0.0017 & 0638 & 1188 \\
\hline $\begin{array}{l}\text { Kiowa_1481-76.FIN2 } 2 \\
\text { Kiowa } 1481 \text { 120.FIN2 }\end{array}$ & $\begin{array}{r}173 \\
233\end{array}$ & $\begin{array}{l}0.64 \\
0.80\end{array}$ & $\begin{array}{l}0.0809 \\
0.0817\end{array}$ & 0.00065 & 1.92 & 0.018 & 0.1717 & $\begin{array}{r}0.0013 \\
0.0175\end{array}$ & 0.53961 & 1085 \\
\hline $\begin{array}{l}\text { Kiowa_1481_120.FIN2 } \\
\text { Kiowa 1481 31FIN2 }\end{array}$ & $\begin{array}{r}23.3 \\
132.3\end{array}$ & 0.80 & 0.0817 & $\begin{array}{r}0.0013 \\
0.00055\end{array}$ & 2.071 & 0.028 & 0.1835 & 0.00175 & 0.18861 & 1138 \\
\hline 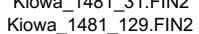 & $\begin{array}{r}132.3 \\
85.7\end{array}$ & $\begin{array}{l}0.81 \\
0.87\end{array}$ & 0.0821 & $\begin{array}{l}0.00055 \\
0.0006\end{array}$ & $\begin{array}{l}2.289 \\
2.371\end{array}$ & $\begin{array}{l}0.075 \\
0.018\end{array}$ & $\begin{array}{l}0.2005 \\
0.2093\end{array}$ & $\begin{array}{l}0.00095 \\
0.0013\end{array}$ & $\begin{array}{l}0.4656 \\
0.4642\end{array}$ & $\begin{array}{l}1207 \\
1233\end{array}$ \\
\hline Kiowa_1481_104.FIN2 & 44.6 & 1.63 & $0.08>>2>-1$ & 0.00125 & 2.064 & 0.0345 & 0.18 & 0.00185 & 0.29006 & 1128 \\
\hline Kiowa_1481_39.FIN2 & 24.09 & 1.38 & $0.08<>>2$ & 0.00195 & 2.236 & 0.04 & 0.1891 & 0.00255 & 0.12329 & 1189 \\
\hline Kiowa_1481_139.FIN2 & 62 & 0.48 & 0.08 & 0.00105 & 2.22 & 0.0 & 0.184 & 0.00205 & 0.62555 & 1180 \\
\hline Kiowa_1481_96.FIN2 & 275 & 0.30 & 0.089 & 0.000 & 3.0 & 0.0 & 0.2 & 0.0011 & 0.53086 & 1413.2 \\
\hline Kiowa_1481_97.FIN2 & 40.3 & 0.65 & & & 3.29 & 0.0 & 0.2 & 0.003 & 0.54671 & 1478 \\
\hline Kiowa_1481_137.FIN & 91.7 & 1.08 & & 0.0 & 2.42 & 0.02 & & 0.00135 & 0.4139 & 1249 \\
\hline Kiowa__1481_57.FIN & 1090 & 0.1 & & & 3.1 & 0.0 & & 0.0 & & 1432 \\
\hline Kiowa_1481_102.F & 96.8 & 0. & & & & & 0.2 & 0.00125 & 0.49637 & 1360 \\
\hline Kiowa_1481_131.F & 867 & 0. & & & 2.9 & 0.0 & & 0.0 & & 1381 \\
\hline Nā_1481__32.FI & 149.4 & 0. & & & & & & & & 1385 \\
\hline Kiowa_1481_68.FI & 750 & 0. & & & & & & 018 & & 1270 \\
\hline Kiowa_1481_44.FI & 291 & 0.6 & 0.091 & & & & & & 224 & 1400.5 \\
\hline Kiowa_1481_59.FIN2 & 349 & 0.09 & & & & 0.0225 & & 0.00175 & 0.45481 & 1399 \\
\hline Kiowa_1481_56.FIN2r & 1400 & 0.07 & & 0.00125 & & 0.0465 & & 0.0033 & 0.61942 & 1420 \\
\hline Kiowa_1481_62.FIN2 & 136 & 1.63 & 0.0929 & 0.00065 & 3.274 & 0.027 & 0.2528 & 0.0015 & 0.54584 & 1472 \\
\hline Kiowa $14811^{-60}$ 60.FIN2 & 156 & 0.97 & 0.0935 & 0.00105 & 2.946 & 0.0495 & 0.2258 & 0.00285 & 0.7607 & 1387 \\
\hline
\end{tabular}

\begin{tabular}{|c|c|c|c|}
\hline 9.5 & 77 & 3.05 & 210 \\
\hline 1.65 & 90.7 & 0.65 & 189 \\
\hline 2.2 & 107.3 & 0.9 & $\begin{array}{l}138 \\
313\end{array}$ \\
\hline 4 & 159 & $\begin{array}{l}1.45 \\
1.55\end{array}$ & $\begin{array}{l}313 \\
179\end{array}$ \\
\hline 2.2 & 165.9 & $\begin{array}{r}1.65 \\
13\end{array}$ & 179 \\
\hline 2.7 & 173.2 & $\begin{array}{r}1.3 \\
1.85\end{array}$ & 177 \\
\hline 3.3 & 187.9 & $\begin{array}{r}1.85 \\
15\end{array}$ & 240 \\
\hline 4.85 & 203.5 & $\begin{array}{l}1.5 \\
14\end{array}$ & $\begin{array}{l}424 \\
204\end{array}$ \\
\hline $\begin{array}{r}2.35 \\
3.3\end{array}$ & $\begin{array}{l}205.7 \\
2029\end{array}$ & $\begin{array}{r}1.4 \\
2\end{array}$ & $\begin{array}{l}204 \\
224\end{array}$ \\
\hline $\begin{array}{l}3.3 \\
.5\end{array}$ & $\begin{array}{l}229.2 \\
275\end{array}$ & $\begin{array}{r}2 \\
1.45\end{array}$ & $\begin{array}{l}241 \\
296\end{array}$ \\
\hline $\begin{array}{r}2.5 \\
3.25\end{array}$ & $\begin{array}{r}275 \\
420.9\end{array}$ & $\begin{array}{l}1.45 \\
2.25\end{array}$ & 412 \\
\hline 3.95 & 439.3 & 4.15 & 472 \\
\hline & 464.6 & 4 & 552 \\
\hline 4.8 & 471.3 & 3.65 & \\
\hline 5.5 & 473 & 6.5 & \\
\hline 4.25 & 474.5 & 3.05 & 508 \\
\hline 6 & 476.8 & 3.5 & \\
\hline 4.2 & 480.2 & 2.65 & \\
\hline 5 & 485.2 & 3.15 & \\
\hline 4.7 & 494 & 5 & \\
\hline 4.75 & 496.8 & 3.35 & \\
\hline 4.35 & 498.3 & 2.45 & \\
\hline 2.55 & 506 & 2.15 & \\
\hline 4.75 & 513.3 & 3.15 & \\
\hline 4.35 & 517 & 4.05 & $\begin{array}{l}429 \\
456\end{array}$ \\
\hline $\begin{array}{r}5 \\
4.85\end{array}$ & $\begin{array}{r}10.4 \\
591\end{array}$ & r. $\begin{array}{r}0.05 \\
5\end{array}$ & \\
\hline 7.5 & 660 & 5 & \\
\hline 11 & 732 & 10 & 1113 \\
\hline 6.5 & 752 & 5.5 & \\
\hline 8 & 1004 & 9.5 & 916 \\
\hline 7 & 867 & 6 & $\begin{array}{r}966 \\
\end{array}$ \\
\hline & 1002 & 6.5 & $\begin{array}{l}1016 \\
1045\end{array}$ \\
\hline 6.5 & 992 & 5 & 1045 \\
\hline 3.7 & $\begin{array}{l}966.9 \\
1139\end{array}$ & $\begin{array}{r}4.15 \\
75\end{array}$ & $\begin{array}{l}1051 \\
1055\end{array}$ \\
\hline $\begin{array}{r}8 \\
65\end{array}$ & $\begin{array}{r}1139 \\
993\end{array}$ & $\begin{array}{l}7.5 \\
6.5\end{array}$ & $\begin{array}{l}1050 \\
1062\end{array}$ \\
\hline $\begin{array}{l}6.5 \\
8.5\end{array}$ & $\begin{array}{l}993 \\
975\end{array}$ & $\begin{array}{r}.5 \\
8\end{array}$ & 1063 \\
\hline 5.5 & 1051 & 6 & 1066 \\
\hline 4.7 & 1063 & 5.5 & 106 \\
\hline 6.5 & 1043 & 7.5 & 57 \\
\hline & 1054 & 9.5 & 07 \\
\hline 5.5 & 1025 & 6 & 07 \\
\hline 5 & 1044 & 5.5 & 08 \\
\hline 6 & 1010 & 7 & 109 \\
\hline & 1007 & 10 & 109 \\
\hline 4.9 & 1021.4 & 4.65 & \\
\hline 6 & 990 & 6.5 & \\
\hline 9.5 & 943 & 7.5 & \\
\hline 10 & 994 & 13 & \\
\hline 10 & 995 & 9.5 & \\
\hline 8.5 & 971 & 8.5 & \\
\hline . & 934 & 10 & \\
\hline 10 & 912 & 11.5 & \\
\hline 5.5 & 1178 & 9 & \\
\hline 6 & $\begin{array}{l}1021 \\
1085\end{array}$ & 7 & 1206 \\
\hline 9.5 & 1085 & 9.5 & \\
\hline $\begin{array}{l}5.5 \\
5 .\end{array}$ & 1178 & 5 & \\
\hline 5.5 & $\begin{array}{l}1224 \\
1066\end{array}$ & 7 & \\
\hline 15 & $\begin{array}{l}1066 \\
1115\end{array}$ & $\begin{array}{l}10 \\
14\end{array}$ & 27 \\
\hline $\begin{array}{l}15.5 \\
10.5\end{array}$ & $\begin{array}{l}1115 \\
1088\end{array}$ & $\begin{array}{r}14 \\
115\end{array}$ & \\
\hline $\begin{array}{l}10.5 \\
3.55\end{array}$ & 1428 & $\begin{array}{r}11.5 \\
5.5\end{array}$ & \\
\hline $\begin{array}{l}3.55 \\
11\end{array}$ & 1530 & 15 & $\begin{array}{l}1419 \\
1426\end{array}$ \\
\hline 8 & 1138 & 7.5 & \\
\hline 9.5 & 1421 & 15 & \\
\hline 5.5 & 1335 & 6.5 & 43 \\
\hline 7.5 & 1337 & & \\
\hline 8 & 1327 & 13.5 & \\
\hline 6.5 & 1161 & 9.5 & \\
\hline 4.8 & 1354 & 6 & \\
\hline & 1351 & 9 & \\
\hline 11.5 & 1386 & 17 & 5 \\
\hline 6.5 & 1452 & 8 & \\
\hline
\end{tabular}

\begin{tabular}{|c|c|c|c|}
\hline 215 & 77.0 & 31 & 8.3 \\
\hline 36.5 & 90.7 & 0.7 & 6.7 \\
\hline 43 & 107.3 & 0.9 & -0.1 \\
\hline 49.5 & 159.0 & 1.5 & 7.4 \\
\hline 26.5 & 165.9 & 1.7 & 1.4 \\
\hline 33.5 & 173.2 & 1.3 & 1.6 \\
\hline 35 & 187.9 & 1.9 & 4.8 \\
\hline 48 & 203.5 & 1.5 & 10.8 \\
\hline 26.5 & 205.7 & 1.4 & 0.9 \\
\hline 34 & 229.2 & 2.0 & 3.0 \\
\hline 23 & 275.0 & 1.5 & 3.8 \\
\hline 20 & 420.9 & 2.3 & 0.4 \\
\hline 20.5 & 439.3 & 4.2 & 4.3 \\
\hline 22.5 & 464.6 & 4.0 & 6.0 \\
\hline 25 & 471.3 & 3.7 & 5.0 \\
\hline 23 & 473.0 & 6.5 & 3.5 \\
\hline 24 & 474.5 & 3.1 & 2.4 \\
\hline 34 & 476.8 & 3.5 & $\begin{array}{l}2.3 \\
2.3\end{array}$ \\
\hline $\begin{array}{l}23 \\
28\end{array}$ & 480.2 & 2.7 & 2.3 \\
\hline $\begin{array}{r}28 \\
165\end{array}$ & 485.2 & $\begin{array}{l}3.2 \\
50\end{array}$ & 0.8 \\
\hline $\begin{array}{r}16.5 \\
26\end{array}$ & $\begin{array}{r}494.0 \\
4968\end{array}$ & $\begin{array}{l}5.0 \\
3.4\end{array}$ & $\begin{array}{l}-0.2 \\
0.3\end{array}$ \\
\hline 23.5 & $\begin{array}{r}490.0 \\
498.3\end{array}$ & $\begin{array}{l}2.4 \\
2.5\end{array}$ & $\begin{array}{l}0.3 \\
-2.1\end{array}$ \\
\hline 13 & 506.0 & 2.2 & -0.3 \\
\hline 24.5 & 513.3 & 3.2 & 0.5 \\
\hline 22 & 517.0 & 4.1 & -2.5 \\
\hline 27 & 518.4 & 3.6 & -2.0 \\
\hline 20.5 & 591.0 & 5.0 & 1.7 \\
\hline 23 & 660.0 & 5.0 & 3.8 \\
\hline 38 & 732.0 & 10.0 & 12.1 \\
\hline 19 & 752.0 & $\begin{array}{r}5.5 \\
\end{array}$ & 8.0 \\
\hline 23.5 & 916.0 & 23.5 & -9.6 \\
\hline 24 & 966.0 & 24.0 & $\begin{array}{l}10.2 \\
1.4\end{array}$ \\
\hline 22 & 1016.0 & 22.0 & $\begin{array}{l}1.4 \\
51\end{array}$ \\
\hline 21 & 1045.0 & 21.0 & 5.1 \\
\hline 12.5 & 1051.0 & 12.5 & 8.0 \\
\hline 25 & 1055.0 & 25.0 & -8.0 \\
\hline 20.5 & 1062.0 & 20.5 & 6.5 \\
\hline 28 & 1063.0 & 28.0 & 8.3 \\
\hline 16.5 & 1066.0 & 16.5 & 1.4 \\
\hline 13 & 1068.0 & 13.0 & 0.5 \\
\hline 16.5 & 1070.0 & 16.5 & 2.5 \\
\hline 16.5 & 1078.0 & $\begin{array}{l}16.5 \\
\end{array}$ & 2.2 \\
\hline 15 & 1079.0 & 15.0 & 5.0 \\
\hline $\begin{array}{r}\begin{array}{r}4.5 \\
16\end{array}\end{array}$ & 1081.0 & $\begin{array}{l}14.5 \\
\end{array}$ & $\begin{array}{l}3.4 \\
7.3\end{array}$ \\
\hline $\begin{array}{r}16 \\
20.5\end{array}$ & $\begin{array}{l}109000 \\
1\end{array}$ & $\begin{aligned} & 16.0 \\
& 20.0\end{aligned}$ & \\
\hline 12.5 & $\begin{array}{l}1095.0 \\
10\end{array}$ & $\begin{array}{l}20.5 \\
12.5\end{array}$ & $\begin{array}{l}8.0 \\
6.7\end{array}$ \\
\hline 17.5 & 1096.0 & 17.5 & 9.7 \\
\hline 30.5 & 1104.0 & 30.5 & \\
\hline 19.5 & 1132.0 & 19.5 & 12.2 \\
\hline 28.5 & 1135.0 & 28.5 & 12.3 \\
\hline 21.5 & 1158.0 & 21.5 & \\
\hline 17.5 & 1160.0 & 17.5 & 19.5 \\
\hline 31.5 & 1180.0 & 31.5 & 22. \\
\hline 12.5 & 1186.0 & 12.5 & 0.7 \\
\hline 16 & 1206.0 & 16.0 & 15.3 \\
\hline 30 & 1214.0 & $\begin{array}{r}30.0 \\
\end{array}$ & 10.6 \\
\hline 13.5 & 1220.0 & 13.5 & 3.4 \\
\hline 14.5 & 1236.0 & 14.5 & 1.0 \\
\hline 29.5 & 1272.0 & 29.5 & 16.2 \\
\hline 47 & 1292.0 & 47.0 & 13.7 \\
\hline 23.5 & 1350.0 & 23.5 & 19.4 \\
\hline 8 & 1419.0 & 8.0 & 0.0 \\
\hline 24 & 1426.0 & 24.0 & -7.3 \\
\hline 20.5 & 1431.0 & 20.5 & 20.5 \\
\hline 19 & 1432.0 & 19.0 & 0.8 \\
\hline 12.5 & 1439.0 & $\begin{array}{l}12.5 \\
\end{array}$ & 7.2 \\
\hline $\begin{array}{l}17.5 \\
155\end{array}$ & 1445.0 & $\begin{array}{l}17.5 \\
15.5\end{array}$ & \\
\hline 15.5 & 1451.0 & $\begin{array}{r}15.5 \\
\end{array}$ & $\begin{array}{r}8.5 \\
\end{array}$ \\
\hline $\begin{array}{r}9 \\
85\end{array}$ & 1455.0 & $\begin{array}{r}9.0 \\
8.0\end{array}$ & 20.2 \\
\hline $\begin{array}{l}8.5 \\
15.5\end{array}$ & 1458.0 & $\begin{array}{l}8.5 \\
155\end{array}$ & $\begin{array}{l}7.1 \\
7\end{array}$ \\
\hline 15.5 & 1458.0 & $\begin{array}{l}15.5 \\
250\end{array}$ & .3 \\
\hline 13.5 & 1478.0 & 13.5 & $\begin{array}{l}\text {. } \\
1.8\end{array}$ \\
\hline 21.5 & 1486.0 & 21.5 & \\
\hline
\end{tabular}




\begin{tabular}{|c|c|c|c|c|c|c|c|c|c|c|c|c|c|c|c|c|c|c|c|}
\hline Kiowa_1481_69.FIN2 & 298 & 1.14 & 0.095 & 0.00075 & 2.398 & 0.024 & 0.1841 & 0.002 & 0.69449 & 1240 & 7.5 & 1088 & 11 & 1522 & 15 & 1522.0 & 15.0 & 28.5 & \\
\hline Kiowa_1481_5.FIN2 & 417 & 0.26 & 0.09844 & 0.000455 & 3.848 & 0.0225 & 0.2819 & 0.00145 & 0.66801 & 1601.2 & 4.7 & 1600 & 7.5 & 1591 & 8.5 & 1591.0 & 8.5 & -0.6 & \\
\hline Kiowa_1481_47.FIN2 & 236 & 0.28 & 0.0993 & 0.0007 & 3.525 & 0.0295 & 0.2563 & 0.00215 & 0.63937 & 1530 & 6.5 & 1470 & 11 & 1604 & 13 & 1604.0 & 13.0 & 8.4 & \\
\hline Kiowa_1481_18.FIN2 & 544 & 0.30 & 0.1005 & 0.00055 & 4.212 & 0.0295 & 0.2933 & 0.00215 & 0.70776 & 1674 & 6 & 1656 & 10.5 & 1627 & 10 & 1627.0 & 10.0 & -1.8 & \\
\hline Kiowa_1481_66.FIN2 & 224 & 1.40 & 0.1009 & 0.0006 & 4.076 & 0.0285 & 0.2916 & 0.0018 & 0.61005 & 1647 & 5.5 & 1649 & 9 & 1638 & 10.5 & 1638.0 & 10.5 & -0.7 & \\
\hline Kiowa_1481_14.FIN2 & 258 & 0.19 & 0.1015 & 0.00075 & 4.058 & 0.0415 & 0.2814 & 0.0026 & 0.71888 & 1643 & 8.5 & 1596 & 13 & 1645 & 14 & 1645.0 & 14.0 & 3.0 & \\
\hline Kiowa_1481_116.FIN2 & 108 & 1.44 & 0.1014 & 0.0006 & 3.957 & 0.031 & 0.282 & 0.002 & 0.70624 & 1622 & 6.5 & 1600 & 10 & 1646 & 11 & 1646.0 & 11.0 & 2.8 & \\
\hline Kiowa_1481_68.FIN2C & 206 & 0.42 & 0.1017 & 0.0013 & 3.67 & 0.07 & 0.2611 & 0.00355 & 0.74457 & 1563 & 15 & 1495 & 18 & 1652 & 24 & 1652.0 & 24.0 & 9.5 & Core \\
\hline Kiowa_1481_37.FIN2 & 94.1 & 1.57 & 0.102 & 0.00085 & 3.449 & 0.045 & 0.2427 & 0.00305 & 0.78877 & 1510 & 10 & 1402 & 16.5 & 1653 & 15.5 & 1653.0 & 15.5 & 15.2 & \\
\hline Kiowa_1481_45.FIN2 & 346 & 0.19 & 0.102 & 0.00055 & 3.921 & 0.0365 & 0.2761 & 0.00175 & 0.83524 & 1616 & 7.5 & 1571 & 9 & 1657 & 10 & 1657.0 & 10.0 & 5.2 & \\
\hline Kiowa_1481_57.FIN2c & 189.6 & 0.41 & 0.1019 & 0.00055 & 3.823 & 0.0215 & 0.2711 & 0.0016 & 0.50671 & 1599.6 & 4.85 & 1546 & 8 & 1659 & 10 & 1659.0 & 10.0 & 6.8 & Core \\
\hline Kiowa_1481_105.FIN2 & 479 & 0.29 & 0.1026 & 0.00085 & 3.73 & 0.05 & 0.2727 & 0.00395 & 0.82266 & 1578 & 11.5 & 1550 & 20 & 1664 & 15 & 1664.0 & 15.0 & 6.9 & \\
\hline Kiowa_1481_83.FIN2 & 185 & 0.29 & 0.1031 & 0.00075 & 3.7 & 0.046 & 0.2582 & 0.003 & 0.82599 & 1564 & 10 & 1478 & 15 & 1674 & 13.5 & 1674.0 & 13.5 & 11.7 & \\
\hline Kiowa_1481_122.FIN2 & 333.1 & 0.25 & 0.10289 & 0.00044 & 3.861 & 0.023 & 0.2696 & 0.0016 & 0.69454 & 1603.8 & 4.8 & 1538 & 8 & 1674 & 8 & 1674.0 & 8.0 & 8.1 & \\
\hline Kiowa_1481_58.FIN2 & 159 & 0.32 & 0.1031 & 0.00055 & 4.033 & 0.0255 & 0.28 & 0.00165 & 0.62426 & 1640 & 5 & 1591 & 8.5 & 1679 & 10.5 & 1679.0 & 10.5 & 5.2 & \\
\hline Kiowa_1481_86.FIN2 & 240.4 & 0.78 & 0.103 & 0.0006 & 4.349 & 0.0355 & 0.3033 & 0.0024 & 0.71234 & 1699 & 7 & 170 & 12 & $168 \mathrm{r}$ & 11 & 1682.0 & 11.0 & -1.4 & \\
\hline Kiowa_1481_106.FIN2 & 306 & 0.68 & 0.10344 & 0.00045 & 3.85 & 0.0205 & 0.2751 & 0.00145 & 0.65669 & 1602.3 & 4.35 & 15 & 7 & 16 & 8 & 1684.0 & 8. & 7. & \\
\hline Kiowa_1481_131.FIN2C & 68.2 & 0.22 & 0.1039 & 0.0011 & 3.553 & 0.0385 & 0.2483 & 0.0021 & 0.47289 & 1539 & 9 & 142 & 11 & 16 & 19.5 & 1684.0 & 19.5 & 15.1 & Core \\
\hline Kiowa 1481 4.FIN2 & 149 & 0.23 & 0.1037 & 0.0006 & 4.166 & 0.0265 & 0.2901 & 0.0015 & 0.5278 & 1668.2 & 4.9 & 164 & 7.5 & 168 & 11 & 1686.0 & 11.0 & 27 & \\
\hline Kiowa 1481 132.FIN2 & 384 & 0.16 & 0.1037 & 0.0005 & 4.206 & 0.025 & 0.2931 & 0.0016 & 0.62513 & 1673.4 & 4.9 & 1656 & 8 & 1687 & 9 & 1687.0 & 9.0 & 1.8 & \\
\hline Kiowa 1481 29.FIN2 & 223 & 0.49 & 0.1039 & 0.00055 & 4.267 & 0.026 & 0.2912 & 0.0018 & 0.65487 & 1687 & 5 & 1647 & 9 & 1692 & 9 & 1692.0 & 9.0 & 2.7 & \\
\hline Kiowa_1481_22.FIN2 & 314 & 0.55 & 0.1041 & 0.0006 & 4.023 & 0.0315 & 0.2711 & 0.002 & 0.73999 & 1637 & 6.5 & 1545 & 10 & 1693 & 10.5 & 1693.0 & 10.5 & 8.7 & \\
\hline Kiowa_1481_43.FIN2C & 117.1 & 0.42 & 0.1043 & 0.00075 & 3.921 & 0.041 & 0.2711 & 0.0028 & 0.76905 & 1615 & 8.5 & 1545 & 14 & 1698 & 13 & 1698.0 & 13.0 & 9.0 & Core \\
\hline Kiowa_1481_133.FIN2 & 161 & 0.52 & 0.1043 & 0.0006 & 4.198 & 0.0345 & 0.2903 & 0.00215 & 0.71955 & 1670 & 7 & 1642 & 10.5 & 1700 & 10.5 & 1700.0 & 10.5 & 3.4 & \\
\hline Kiowa_1481_24.FIN2 & 157 & 0.59 & 0.1047 & 0.00065 & 4.062 & 0.0245 & 0.2737 & 0.00195 & 0.55318 & 1644.8 & 4.95 & 1559 & 10 & 1702 & 12 & 1702.0 & 12.0 & 8.4 & \\
\hline Kiowa_1481_30.FIN2 & 241 & 0.30 & 0.105 & 0.0007 & 3.826 & 0.0475 & 0.258 & 0.00275 & 0.84569 & 1591 & 10 & 1477 & 14 & 1706 & 12.5 & 1706.0 & 12.5 & 13.4 & \\
\hline Kiowa_1481_74.FIN2 & 334 & 1.63 & 0.10519 & 0.000395 & 4.136 & 0.0205 & 0.2844 & 0.00125 & 0.63922 & 1660.1 & 4.05 & 1613 & 6 & 1715 & 7 & 1715.0 & 7.0 & 5.9 & \\
\hline Kiowa_1481_117.FIN2 & 116.3 & 0.56 & 0.1061 & 0.0008 & 4.18 & 0.038 & 0.2834 & 0.00245 & 0.68386 & 1669 & 7.5 & 1610 & 13 & 1726 & 13.5 & 1726.0 & 13.5 & 6.7 & \\
\hline Kiowa_1481_10.FIN2 & 690 & 0.23 & 0.1059 & 0.0005 & 5.41 & 0.055 & 0.3602 & 0.0032 & 0.87074 & 1881 & 9 & 1985 & 15.5 & 1728 & 9 & 1728.0 & 9.0 & -14.9 & \\
\hline Kiowa_1481_40.FIN2 & 117.1 & 0.62 & 0.1062 & 0.0008 & 4.521 & 0.045 & 0.3066 & 0.00305 & 0.757 & 1730 & 8 & 1722 & 15 & 1729 & 14 & 1729.0 & 14.0 & 0.4 & \\
\hline Kiowa_1481_71.FIN2 & 408 & 0.77 & 0.1061 & 0.00055 & 4.336 & 0.0235 & 0.2977 & 0.00145 & 0.51213 & 1698.9 & 4.4 & 1679 & 7 & 1729 & 9 & 1729.0 & 9.0 & 2.9 & \\
\hline Kiowa_1481_101.FIN2 & 293 & 0.29 & 0.1061 & 0.0008 & 4.377 & 0.0485 & 0.3056 & 0.00345 & 0.72424 & 1705 & 9.5 & 1718 & 17 & 1729 & 14.5 & 1729.0 & 14.5 & 0.6 & \\
\hline Kiowa_1481_56.FIN2C & 294 & 0.39 & 0.107 & 0.00075 & 4.159 & 0.033 & 0.2794 & 0.00195 & 0.55488 & 1665 & 6.5 & 1588 & 10 & 1744 & 13 & 1744.0 & 13.0 & 8.9 & Core \\
\hline Kiowa_1481_61.FIN2 & 173 & 0.61 & 0.1073 & 0.00085 & 3.73 & 0.055 & 0.2508 & 0.00345 & 0.87049 & 1578 & 11 & 1439 & 18 & 1744 & 14 & 1744.0 & 14.0 & 17.5 & \\
\hline Kiowa_1481_72.FIN2 & 502 & 0.70 & 0.1071 & 0.0005 & 4.385 & 0.0245 & 0.2962 & 0.0017 & 0.6648 & 1707.9 & 4.65 & 1672 & 8.5 & 1747 & 8.5 & 1747.0 & 8.5 & 4.3 & \\
\hline Kiowa_1481_23.FIN2 & 67.7 & 0.66 & 0.1075 & 0.0007 & 4.758 & 0.0335 & 0.3092 & 0.00165 & 0.44543 & 1775 & 6 & 1736 & 8 & 1753 & 12.5 & 1753.0 & 12.5 & 1.0 & \\
\hline Kiowa_1481_119.FIN2 & 347 & 0.16 & 0.1084 & 0.001 & 4.41 & 0.06 & 0.2928 & 0.0038 & 0.75712 & 1707 & 11.5 & 1656 & 18.5 & 1762 & 16.5 & 1762.0 & 16.5 & 6.0 & \\
\hline Kiowa_1481_7.FIN2 & 230.3 & 0.46 & 0.1082 & 0.00055 & 3.484 & 0.036 & 0.2298 & 0.00195 & 0.87343 & 1518 & 8.5 & 1332 & 10 & 1765 & 9.5 & 1765.0 & 9.5 & 24.5 & \\
\hline Kiowa_1481_99.FIN2 & 69.7 & 0.65 & 0.1105 & 0.0008 & 4.668 & 0.0385 & 0.3128 & 0.0026 & 0.64384 & 1761 & 6.5 & 1753 & 13 & 1799 & 13 & 1799.0 & 13.0 & 2.6 & \\
\hline Kiowa_1481_100.FIN2 & 152.2 & 1.29 & 0.1142 & 0.00055 & 5.293 & 0.028 & 0.3438 & 0.00185 & 0.57124 & 1866.3 & 4.5 & 1906 & 8.5 & 1862 & 9 & 1862.0 & 9.0 & -2.4 & \\
\hline Kiowa_1481_28.FIN2 & 264 & 0.38 & 0.1255 & 0.001 & 6.06 & 0.055 & 0.3407 & 0.00265 & 0.53696 & 1983 & 8 & 1888 & 13 & 2025 & 14 & 2025.0 & 14.0 & 6.8 & \\
\hline Kiowa_1481_90.FIN2 & $\begin{array}{l}86.4 \\
257\end{array}$ & 0.88 & 0.1826 & 0.00105 & $\begin{array}{r}12.23 \\
131\end{array}$ & 0.105 & 0.4839 & $\begin{array}{l}0.00275 \\
0.00265\end{array}$ & 0.73098 & 2618 & 8 & 2543 & 12 & 2672 & 9.5 & 2672.0 & 9.5 & 4.8 & \\
\hline Kiowa_1481_21.FIN2 & 257 & 0.49 & $\begin{array}{r}0.186 \\
0.198\end{array}$ & $\begin{array}{r}0.0008 \\
0.00125\end{array}$ & $\begin{array}{r}13.1 \\
1267\end{array}$ & 0.07 & 0.4926 & $\begin{array}{l}0.00265 \\
0.00375\end{array}$ & $\begin{array}{l}0.69906 \\
0.75042\end{array}$ & $\begin{array}{l}2685 \\
2652\end{array}$ & 5 & $\begin{array}{l}2580 \\
2510\end{array}$ & 11.5 & 2704 & $\begin{array}{r}7 \\
105\end{array}$ & 2704.0 & $\begin{array}{l}7.0 \\
105\end{array}$ & 4.6 & \\
\hline Kiowa 1481 25.FIN2 & $\begin{array}{l}94.7 \\
473\end{array}$ & $\begin{array}{l}0.87 \\
0.11\end{array}$ & $\begin{array}{l}0.1928 \\
0.1987\end{array}$ & $\begin{array}{l}0.00105 \\
0.00105\end{array}$ & $\begin{array}{l}12.01 \\
14.26\end{array}$ & $\begin{array}{r}0.115 \\
0.1\end{array}$ & $\begin{array}{l}0.467 \\
0.505\end{array}$ & $\begin{array}{r}0.00375 \\
0.0043\end{array}$ & $\begin{array}{l}0.75042 \\
0.78405\end{array}$ & $\begin{array}{l}2652 \\
2764\end{array}$ & $\begin{array}{r}9 \\
65\end{array}$ & 2510 & $\begin{array}{r}16 \\
185\end{array}$ & 2760 & $\begin{array}{r}10.5 \\
8.5\end{array}$ & 2760.0 & 10.5 & 9.1 & \\
\hline Kiowa 1481 35 FIN2 & 291 & 0.92 & 0.0468 & 0.0012 & 0.0605 & 0.00155 & 0.00919 & 0.0001 & 0.18189 & $\begin{array}{r}2 / 64 \\
59.5\end{array}$ & $\begin{array}{l}6.5 \\
1.5\end{array}$ & $\begin{aligned} 2631 \\
59\end{aligned}$ & $\begin{array}{l}18.5 \\
0.65\end{array}$ & $\begin{aligned} 2812 \\
67\end{aligned}$ & $\begin{array}{r}8.5 \\
47.5\end{array}$ & $\begin{array}{l}2812.0 \\
\text { DISCARD }\end{array}$ & $\begin{array}{l}8.5 \\
\text { DISCARD }\end{array}$ & $\begin{array}{l}6.4 \\
0.8\end{array}$ & \\
\hline Kiowa_1481_81.FIN2 & 42.7 & 1.61 & 0.055 & 0.0036 & 0.0656 & 0.004 & 0.00929 & 0.00021 & -0.052511 & 64.3 & 3.85 & 59.6 & 1.35 & 150 & 105 & DISCARD & DISCARD & 7.3 & \\
\hline Kiowa_1481_49.FIN2 & 42.1 & 2.22 & 0.0572 & 0.00355 & 0.0705 & 0.0042 & 0.00937 & 0.000175 & 0.035224 & 68 & 3.95 & 60.1 & 1.15 & 240 & 110 & DISCARD & DISCARD & 11.6 & \\
\hline Kiowa_1481_126.FIN2 & 60.2 & 1.24 & 0.0506 & 0.00235 & 0.066 & 0.003 & 0.00944 & 0.00014 & 0.040022 & 65 & 2.95 & 60.8 & 0.9 & 160 & 85 & DISCARD & DISCARD & 6.5 & \\
\hline Kiowa_1481_78.FIN2 & 70.5 & 1.78 & 0.0443 & 0.0024 & 0.0589 & 0.0032 & 0.00968 & 0.00015 & 0.12194 & 57.4 & 3.05 & 62.1 & 0.95 & -90 & 85 & DISCARD & DISCARD & -8.2 & \\
\hline Kiowa_1481_79.FIN2 & 38 & 1.80 & 0.0561 & 0.00395 & 0.073 & 0.005 & 0.00976 & 0.000185 & 0.15225 & 70.1 & 4.8 & 62.6 & 1.15 & 150 & 120 & DISCARD & DISCARD & 10.7 & \\
\hline Kiowa_1481_51.FIN2 & 31.01 & 1.84 & 0.0535 & 0.00375 & 0.0681 & 0.0043 & 0.0098 & 0.00022 & -0.054653 & 65.7 & 4.05 & 62.8 & 1.4 & 110 & 1115 & DISCARD & DISGARD & 4.4 & \\
\hline Kiowa_1481_77.FIN2 & 45.1 & 1.82 & 0.0451 & 0.00305 & 0.0602 & 0.004 & 0.00988 & 0.00021 & 0.065814 & 58.3 & 3.8 & 63.3 & 1.35 & 70 & 110 & DISCARD & DISGARD & -8.6 & \\
\hline Kiowa_1481_55.FIN2 & 24.8 & 1.71 & 0.0514 & 0.00435 & 0.068 & 0.0055 & 0.01005 & 0.000245 & 0.1151 & 65 & 5 & 64.4 & 1.55 & -40 & 125 & DISCARD & DISGARD & 0.9 & \\
\hline Kiowa_1481_112.FIN2 & 75 & 4.34 & 0.0526 & 0.0024 & 0.0717 & 0.00335 & 0.01008 & 0.00014 & 0.3096 & 69.6 & 3.1 & 64.6 & 0.9 & 210 & 80 & DISCARD & DISGARD & 7.2 & \\
\hline Kiowa_1481_107.FIN2 & 22.14 & 1.03 & 0.0543 & 0.0046 & 0.069 & 0.0055 & 0.01027 & 0.000245 & 0.13846 & 65.8 & 4.9 & 65.9 & 1.55 & 50 & 125 & DISCARD & DISGARD & 0.2 & \\
\hline Kiowa_1481_43.FIN2r & 96.5 & 0.82 & 0.0536 & 0.00445 & 0.077 & 0.005 & 0.01029 & 0.00035 & -0.041459 & 75 & 5 & 66 & 2.25 & 350 & 170 & DISCARD & DISGARD & 12.0 & Rim \\
\hline Kiowa_1481_21.FIA & 98.4 & 2.04 & 0.0439 & 0.0019 & 0.0615 & 0.0024 & 0.01037 & 0.000135 & 0.017009 & 60 & 2.35 & 66.5 & 0.85 & -80 & 70 & & DISCARD & -10.5 & \\
\hline Kiowa_1 & 114 & 1.43 & 0.0481 & 0.00 & 0.0699 & 0.0027 & 0.01041 & 0.00015 & -0.022832 & 68 & 2.55 & 66.7 & 0.95 & 140 & 75 & & DISCARD & 2.1 & \\
\hline Kiowa_1481_88.FIN2 & 118 & 1.43 & 0.0479 & 0.0017 & 0.0703 & 0.00245 & 0.01063 & 0.00013 & 0.050646 & 68.6 & 2.3 & 68 & 0.85 & 120 & 65 & DISC & DISCARD & 0.7 & \\
\hline Kiowa_1481_13.FIN2 & 224 & 0.59 & 0.0484 & 0.00135 & 0.0726 & 0.0019 & 0.01065 & 0.000135 & 0.16646 & 71.4 & 1.9 & 68.3 & 0.85 & 140 & 55 & DISC & DISCARD & 4.3 & \\
\hline Kiowa_1481_115.FIN2 & 119.2 & 0.34 & 0.0477 & 0.00145 & 0.0696 & 0.0020 & 0.010 & 0.00011 & -0.028843 & & 1.95 & 68.5 & 0.7 & 90 & 55 & DISC & DISCARD & -0.7 & \\
\hline Kiowa_1481_9.FIN2 & 223 & 0.44 & 0.0767 & 0.0011 & 0.632 & 0.0215 & 0.058 & 0.00175 & 0.92503 & 489 & 12.5 & 365 & 10.5 & 1097 & 27 & DISC & \#VALUE! & 25.4 & \\
\hline Kiowa_1481_50.FIN2 & 35.4 & 1.57 & 0.075 & 0.005 & 0.106 & 0.007 & 0.01039 & 0.00024 & 0.078374 & 100 & 6.5 & 66.6 & 1.55 & 710 & 135 & & \#VALUE! & 33.4 & \\
\hline Kiowa_1481_82.FIN2 & 34.6 & 1.62 & 0.0434 & 0.00325 & 0.0553 & 0.00405 & 0.00968 & 0.00023 & 0.027249 & 53.6 & 3.85 & 62.1 & 1.45 & -170 & 110 & & \#VALUE! & -15.9 & \\
\hline \multirow{3}{*}{ Kiowa_1481_124.FIN2 } & 107.9 & 1.45 & 0.107 & 0.0065 & 0.16 & 0.0105 & 0.01046 & 0.000175 & 0.46838 & 145 & 9 & 67.1 & 1.1 & 1150 & 125 & DISCARD & \#VALUE! & 53.7 & \\
\hline & 174 & 1.96 & 0.0781 & 0.00075 & 1.177 & 0.02 & 0.1087 & 0.00175 & 0.82564 & 784 & 9.5 & 664 & 10.5 & 1138 & 19 & DISCARD & \#УALUE! & 75.3 & \\
\hline & & & & internal & & internal & & internal & & 207/235 & internal & $206 / 238$ & internal & \begin{tabular}{|l|}
$207 / 206$ \\
\end{tabular} & internal & Best age & internal & & \\
\hline Analysis ID & \begin{tabular}{|l|}
{$[\mathrm{U}] \mathrm{ppm}$} \\
\end{tabular} & \begin{tabular}{l|l} 
Th/U \\
\end{tabular} & 207/206 & 10 error & $207 / 235$ & $1 \sigma$ error & $206 / 238$ & $1 \sigma$ error & RHO & Age Ma & $1 \sigma$ error & Age (Ma) & $1 \sigma$ error & Age (Ma) & $1 \sigma$ error & (Ma) & $1 \sigma$ error & \% Discordance* & Rim/Core \\
\hline Kiowa_1635_21.FIN2 & 45.2 & 1.52 & 0.0525 & 0.00245 & 0.0752 & 0.00355 & 0.01043 & 0.00016 & 0.088591 & 72.8 & 3.35 & 66.8 & & 200 & 85 & 66.8 & 1.0 & 8.2 & \\
\hline Kiowa_1635_125.FIN2 & 69.8 & 1.34 & 0.0519 & 0.00225 & 0.0758 & 0.0032 & 0.01085 & 0.000155 & 0.11543 & 73.5 & 3 & 69.6 & 1 & 160 & 75 & 69.6 & 1.0 & 5.3 & \\
\hline Kiowa_1635_16.FIN2 & 359 & 0.64 & 0.0492 & 0.00095 & 0.074 & 0.00135 & 0.01098 & 0.000075 & 0.10753 & 72.4 & 1.3 & 70.42 & 0.49 & 148 & 37.5 & 70.4 & 0.5 & 2.7 & \\
\hline Kiowa_1635_15.FIN2 & 218.3 & 0.42 & 0.0473 & 0.00105 & 0.0708 & 0.00145 & 0.01101 & 0.00008 & -0.018914 & 69.3 & 1.35 & 70.6 & 0.5 & 74 & 40.5 & 70.6 & 0.5 & -1.9 & \\
\hline Kiowa_1635_46.FIN2 & 197 & 0.93 & 0.0468 & 0.0011 & 0.0722 & 0.00155 & 0.01126 & 0.000095 & 0.11284 & 70.6 & 1.5 & 72.2 & 0.6 & 53 & 43 & 72.2 & 0.6 & -2.3 & \\
\hline Kiowa_1635_1.FIN2 & 720 & 0.43 & 0.0475 & 0.0005 & 0.0761 & 0.00075 & 0.0116 & 0.000055 & 0.19477 & 74.5 & 0.7 & 74.32 & 0.35 & 81 & 21.5 & 74.3 & 0.4 & 0.2 & \\
\hline
\end{tabular}




\begin{tabular}{|c|c|c|c|c|c|c|c|c|c|c|}
\hline Kiowa_1635_114.FIN2 & 1391 & 0.50 & 0.04811 & 0.000455 & 0.0773 & 0.0008 & 0.01161 & 0.000065 & 0.38525 & 75.6 \\
\hline Kiowa_1635_123.FIN2 & 107 & 0.65 & 0.0496 & 0.00155 & 0.0818 & 0.0025 & 0.01184 & 0.00012 & -0.04308 & 79.4 \\
\hline Kiowa_1635_119.FIN2 & 641 & 0.51 & 0.0463 & 0.0006 & 0.0773 & 0.001 & 0.01204 & 0.000085 & 0.29528 & 75.5 \\
\hline Kiowa_1635_33.FIN2 & 173.1 & 1.06 & 0.0497 & 0.0011 & 0.0836 & 0.00175 & 0.01226 & 0.00009 & 0.10213 & 81.3 \\
\hline Kiowa_1635_19.FIN2 & 435 & 1.40 & 0.0493 & 0.0007 & 0.0839 & 0.00125 & 0.01235 & 0.00009 & 0.35084 & 81.7 \\
\hline Kiowa_1635_7.FIN2 & 346.5 & 0.38 & 0.0503 & 0.0012 & 0.0893 & 0.002 & 0.01294 & 0.000125 & 0.093077 & 86.8 \\
\hline Kiowa_1635_48.FIN2 & 444 & 0.59 & 0.0493 & 0.0007 & 0.0897 & 0.0013 & 0.01321 & 0.000095 & 0.17112 & 87.1 \\
\hline Kiowa_1635_135.FIN2 & 164 & 0.63 & 0.0466 & 0.00105 & 0.0871 & 0.00185 & 0.01351 & 0.000135 & 0.14704 & 84.5 \\
\hline Kiowa_1635_104.FIN2 & $\begin{array}{r}579 \\
249\end{array}$ & 0.35 & 0.0487 & 0.00055 & 0.0941 & 0.0011 & 0.01409 & 0.000085 & $\begin{array}{l}0.34254 \\
0.20465\end{array}$ & $\begin{array}{l}91.3 \\
95.9\end{array}-9$ \\
\hline $\begin{array}{l}\text { Kiowa_-1635-78.FIN2 } \\
\text { Kowawa 1635 50.FIN2 }\end{array}$ & $\begin{array}{l}249 \\
472\end{array}$ & $\begin{array}{l}0.69 \\
0.96\end{array}$ & $\begin{array}{l}0.0479 \\
0.0528\end{array}$ & $\begin{array}{l}0.00085 \\
0.00075\end{array}$ & 0.0993 & $\begin{array}{r}0.0019 \\
0.0025\end{array}$ & 0.01494 & 0.000095 & $\begin{array}{r}0.20465 \\
0.3091\end{array}$ & 95.9 \\
\hline $\begin{array}{l}\text { Kiowa_ } 1635 \text {-50.FIN2 } \\
\text { Kiowa } 1635 \text { 18.FIN2 }\end{array}$ & $\begin{array}{l}472 \\
242\end{array}$ & $\begin{array}{l}0.96 \\
1.15\end{array}$ & $\begin{array}{l}0.0528 \\
0.0561\end{array}$ & $\begin{array}{l}0.00075 \\
0.0008\end{array}$ & $\begin{array}{l}0.1731 \\
0.1915\end{array}$ & $\begin{array}{l}0.00255 \\
0.0025\end{array}$ & $\begin{array}{l}0.02399 \\
0.02489\end{array}$ & $\begin{array}{l}0.000185 \\
0.000185\end{array}$ & $\begin{array}{r}0.3091 \\
0.10819\end{array}$ & $\begin{array}{l}161.8 \\
177.7\end{array}$ \\
\hline Kiowa_1635_120.FIN2 & 589 & 1.29 & 0.04978 & 0.00044 & 0.174 & 0.00165 & $\begin{array}{l}0.02489 \\
0.02522\end{array}$ & $\begin{array}{r}0.000185 \\
0.00013\end{array}$ & $\begin{array}{l}0.10819 \\
0.34052\end{array}$ & 116.7 \\
\hline Kiowa_1635_124.FIN2 & 836 & 0.97 & 0.04858 & 0.00039 & 0.1726 & 0.00155 & 0.02568 & 0.000145 & 0.48043 & $\begin{array}{l}102.9 \\
161.9\end{array}$ \\
\hline Kiowa_1635_27.FIN2 & 523 & 0.87 & 0.0499 & 0.00055 & 0.1792 & 0.002 & 0.02586 & 0.00016 & 0.37195 & 167.2 \\
\hline Kiowa_1635_86.FIN2 & 504 & 0.79 & 0.05104 & 0.00044 & 0.1813 & 0.00145 & 0.02612 & 0.000135 & 0.21179 & 169 \\
\hline Kiowa_1635_38.FIN2 & 173.1 & 1.17 & 0.051 & 0.00085 & 0.183 & 0.00285 & 0.02615 & 0.000195 & 0.12065 & 170.2 \\
\hline Kiowa_1635_90.FIN2 & 213 & 1.13 & 0.0568 & 0.00105 & 0.2053 & 0.00415 & 0.0262 & 0.000175 & 0.25051 & 188.7 \\
\hline Kiowa_1635_44.FIN2 & 392 & 0.26 & 0.0532 & 0.00075 & 0.1904 & 0.00315 & 0.02623 & 0.000295 & 0.55302 & 177.1 \\
\hline Kiowa_1635_102.FIN2 & 333 & 1.10 & 0.055 & 0.00065 & 0.1975 & 0.00225 & 0.02627 & 0.00016 & 0.28936 & 182.8 \\
\hline Kiowa_1635_43.FIN2 & 366 & 1.14 & 0.0517 & 0.00055 & 0.2143 & 0.00245 & 0.03007 & 0.000245 & 0.3774 & 196.9 \\
\hline Kiowa_1635_8.FIN2 & 326 & 0.87 & 0.0517 & 0.00055 & 0.2426 & 0.0026 & 0.03402 & 0.000195 & 0.24931 & 220.2 \\
\hline Kiowa_1635_118.FIN2 & 319 & 0.41 & 0.05494 & 0.000465 & 0.4593 & 0.0042 & 0.06053 & 0.00045 & 0.55305 & 383.9 \\
\hline Kiowa_1635_72.FIN2 & 257 & 0.48 & 0.05616 & 0.00048 & 0.5 & 0.00425 & 0.06471 & 0.000285 & 0.24781 & 411.1 \\
\hline Kiowa_1635_126.FIN2 & 238 & 0.62 & 0.0574 & 0.00085 & 0.524 & 0.009 & 0.0663 & 0.00085 & 0.49472 & 425 \\
\hline Kiowa_1635_66.FIN2 & 216 & 0.89 & 0.05481 & 0.000455 & 0.5086 & 0.0043 & 0.06719 & 0.000395 & 0.32065 & 416.9 \\
\hline Kiowa_1635_63.FIN2 & 215.4 & 1.63 & 0.0555 & 0.00047 & 0.5247 & 0.0045 & 0.0683 & 0.000285 & 0.27253 & 427.6 \\
\hline Kiowa_1635_12.FIN2 & 150.7 & 0.94 & 0.0562 & 0.00065 & 0.528 & 0.0055 & 0.06889 & 0.000355 & 0.16119 & 429.2 \\
\hline Kiowa_1635_52.FIN2 & 183.5 & 1.16 & 0.0568 & 0.00055 & 0.5664 & 0.00485 & 0.07334 & 0.000455 & 0.24188 & 455 \\
\hline Kiowa_1635_107.FIN2 & 111.8 & 0.49 & 0.057 & 0.0006 & 0.588 & 0.0065 & 0.07524 & 0.000405 & 0.35623 & 470.4 \\
\hline Kiowa_1635_54.FIN2 & 53.7 & 0.93 & 0.0619 & 0.001 & 0.737 & 0.013 & 0.0865 & 0.0006 & 0.23332 & 558 \\
\hline Kiowa_1635_108.FIN2 & 107.8 & 0.61 & $\begin{array}{r}0.0733 \\
\end{array}$ & 0.0006 & 1.665 & 0.0135 & & 0.0008 & 0.3277 & 993 \\
\hline Kiowa_1635_112.FIN2 & 125.7 & 0.44 & 0.07347 & 0.000495 & 1.7 & 0.0115 & 0.1681 & 0.00085 & 0.39996 & 1007.3 \\
\hline Kiowa_1635-103.FIN2 & $\begin{array}{r}264 \\
558\end{array}$ & 0.37 & $\begin{array}{l}0.07358 \\
0.0741\end{array}$ & 0.00043 & 1.674 & $\begin{array}{l}0.0105 \\
0.019\end{array}$ & 0.1659 & 0.00075 & 0.55066 & 997.4 \\
\hline Kiowa_1635_4.FIN2 & 55.8 & 1.12 & $\begin{array}{l}0.0741 \\
\end{array}$ & $\begin{array}{r}0.0008 \\
0.00055\end{array}$ & $\begin{array}{l}1.716 \\
176\end{array}$ & $\begin{array}{l}0.019 \\
0.013\end{array}$ & 0.1671 & 0.00125 & 0.3428 & 1013 \\
\hline Kiowa_1635_85.FIN2r & $\begin{array}{l}512 \\
131\end{array}$ & 0.01 & $\begin{array}{l}0.0738 \\
0.0743\end{array}$ & $\begin{array}{l}0.00055 \\
0.0005\end{array}$ & $\begin{array}{r}1.76 \\
1.891\end{array}$ & $\begin{array}{r}0.013 \\
0.0145\end{array}$ & $\begin{array}{l}0.175 \\
0.184\end{array}$ & 0.00115 & $\begin{array}{l}0.46466 \\
0.52758\end{array}$ & $\begin{array}{l}1030.1 \\
1076\end{array}$ \\
\hline Kiowa_1635_30.FIN2 & $\begin{array}{r}131 \\
386\end{array}$ & 0.53 & $\begin{array}{l}0.0743 \\
0.0742\end{array}$ & $\begin{array}{r}0.0005 \\
0.00075\end{array}$ & $\begin{array}{r}1.891 \\
1.83\end{array}$ & $\begin{array}{l}0.0145 \\
0.025\end{array}$ & $\begin{array}{r}0.184 \\
0.1804\end{array}$ & $\begin{array}{l}0.00105 \\
0.00175\end{array}$ & $\begin{array}{l}0.52758 \\
0.65721\end{array}$ & $\begin{array}{l}1076 \\
1052\end{array}$ \\
\hline $\begin{array}{l}\text { lowa- } 1635 \text {-91.. } \\
\text { Kiowa } 1635 \text { 3.FIN2 }\end{array}$ & $\begin{array}{r}38.6 \\
1009\end{array}$ & $\begin{array}{l}0.63 \\
0.58\end{array}$ & $\begin{array}{r}0.0742 \\
0.07452\end{array}$ & 0.00075 & $\begin{array}{r}1.83 \\
1858\end{array}$ & $\begin{array}{l}0.025 \\
0.013\end{array}$ & $\begin{array}{l}0.1804 \\
0.1804\end{array}$ & $\begin{array}{l}0.001 / 5 \\
0.0009\end{array}$ & $\begin{array}{l}0.65 / 27 \\
0.44496\end{array}$ & 1052 \\
\hline Kiowa_1635_62.FIN2 & 540 & $\begin{array}{l}0.00 \\
0.03\end{array}$ & 0.07429 & $\begin{array}{l}0.00048 \\
0.00048\end{array}$ & $\begin{array}{l}1.1 .736 \\
1.736\end{array}$ & $\begin{array}{l}0.013 \\
0.012\end{array}$ & $\begin{array}{l}0.1804 \\
0.1693\end{array}$ & $\begin{array}{l}0.0009 \\
0.0009\end{array}$ & $\begin{array}{l}0.44446 \\
0.42882\end{array}$ & $\begin{array}{l}1065.8 \\
1021.2\end{array}$ \\
\hline Kiowa_1635_37.FIN2 & 267 & 0.41 & 0.0753 & 0.0007 & 1.561 & 0.015 & 0.1518 & 0.0016 & 0.60498 & 952 \\
\hline Kiowa_1635_93.FIN2 & 119 & 1.20 & 0.0759 & 0.0005 & 1.841 & 0.015 & 0.1779 & 0.0012 & 0.60652 & 1058 \\
\hline Kiowa_1635_11.FIN2 & 120.3 & 0.82 & 0.0759 & 0.0005 & 1.904 & 0.014 & 0.1829 & 0.00095 & 0.48487 & 1080.7 \\
\hline Kiowa_1635_82.FIN2 & 155.7 & 0.63 & 0.0769 & 0.0007 & 1.939 & 0.0225 & 0.183 & 0.0015 & 0.58488 & 1090 \\
\hline Kiowa_1635_17.FIN2 & 445 & 0.63 & 0.07774 & 0.00042 & 1.984 & 0.0115 & 0.1859 & 0.00095 & 0.53212 & 1108.8 \\
\hline Kiowa_1635_39.FIN2 & 252.5 & 0.39 & 0.07785 & 0.00033 & 2.108 & 0.011 & 0.1958 & 0.0008 & 0.57817 & 1150.5 \\
\hline Kiowa_1635_57.FIN2 & 176 & 0.25 & 0.0789 & 0.00065 & 2.24 & 0.0225 & 0.206 & 0.00185 & 0.58358 & 1190 \\
\hline Kiowa_1635_10.FIN2 & 186 & 0.69 & 0.07893 & 0.00041 & 2.094 & 0.013 & 0.1923 & 0.00085 & 0.53084 & 1145.4 \\
\hline Kiowa_1635_60.FIN2 & 108.3 & 1.00 & 0.0823 & 0.00165 & 1.908 & 0.042 & 0.1732 & 0.0037 & 0.58696 & 1078 \\
\hline Kiowa_1635_76.FIN2 & 426 & 0.99 & 0.0794 & 0.000455 & 2.262 & 0.0 & & 0.00155 & 0.66234 & 1200 \\
\hline Kiowa_1635_22.FII & 309 & 0.65 & 0.07969 & 0.00035 & & 0.01 & & 0.0012 & & 1169.1 \\
\hline Kiowa_1635_105.FI & 71.2 & 0.87 & 0.08 & & & & & & & 1161 \\
\hline Kiowa_1635_14.FI & 200 & 0.73 & $0.08 \mathrm{C}$ & 0.000 & & & & & & 1173.9 \\
\hline Kiowa_163 & 151 & 0.61 & 0.0812 & 0.0 & 2. & & & & & 1213 \\
\hline Kiowa_163 & 244 & 1.02 & & 0.00 & & 0.01 & & 0009 & 8174 & 1212.5 \\
\hline Kiowa_16 & 71.1 & 1.20 & & 0.00 & 2.305 & & 0.2 & 0.0013 & & 1212 \\
\hline Kiowa_1635_100.F & 180.7 & 0.65 & 0.08352 & 0.000415 & & 0.012 & & & 0.45635 & 1259.3 \\
\hline Kiowa_1635_136.FI & 70.2 & 1.55 & 0.0874 & 0.0008 & 3.018 & 0.0245 & 0.2489 & 0.00185 & 4321 & \\
\hline Kiowa_1635_128.FII & $\begin{array}{l}26.83 \\
482\end{array}$ & $\begin{array}{l}1.28 \\
3.34\end{array}$ & 0.0882 & $\begin{array}{r}0.001 \\
0.00065\end{array}$ & 2.733 & 0.0295 & 0.2241 & 0.00125 & 0.19167 & 1337 \\
\hline & $\begin{array}{l}48.2 \\
151\end{array}$ & $\begin{array}{l}3.34 \\
0.52\end{array}$ & 0.0877 & 0.00065 & $\begin{array}{l}2.803 \\
2804\end{array}$ & $\begin{array}{l}0.021 \\
0.023\end{array}$ & 0.2327 & 0.00135 & 0.38027 & 1354 \\
\hline Kiowa_1635_85.FIN2C & $\begin{array}{r}151 \\
1268\end{array}$ & 0.52 & 0.0874 & $\begin{array}{l}0.0007 \\
-0.008\end{array}$ & 2.804 & 0.023 & 0.2345 & $\begin{array}{r}0.0015 \\
\end{array}$ & 0.38233 & 1356 \\
\hline $\begin{array}{l}\text { Kowa-1635-41.FIN2r } \\
\text { Kiow-1635-45.FIN2 }\end{array}$ & $\begin{array}{r}1268 \\
293\end{array}$ & $\begin{array}{l}0.01 \\
0.52\end{array}$ & $\begin{array}{l}0.0893 \\
0.0897\end{array}$ & $\begin{array}{l}0.0008 \\
0.00075\end{array}$ & $\begin{array}{l}2.987 \\
2.832\end{array}$ & $\begin{array}{l}0.028 \\
0.028\end{array}$ & $\begin{array}{l}0.2247 \\
0.2302\end{array}$ & $\begin{array}{l}0.00224 \\
0.00205\end{array}$ & $\begin{array}{l}0.50371 \\
0.64269\end{array}$ & $\begin{array}{l}1402 \\
1360\end{array}$ \\
\hline Kiowa_1635_71.FIN2 & 356 & 0.94 & 0.08931 & 0.000325 & 3.021 & 0.013 & 0.2457 & 0.00095 & 0.58859 & 1412.1 \\
\hline Kiowa_1635_80.FIN2 & 204 & 1.50 & 0.0902 & 0.0006 & 3.023 & 0.0275 & 0.2444 & 0.00185 & 0.64193 & 1410 \\
\hline Kiowa_1635_97.FIN2 & 272 & 1.12 & 0.09009 & 0.000475 & 2.795 & 0.016 & 0.2281 & 0.00115 & 0.56063 & 1354 \\
\hline Kiowa_1635_87.FIN2r & 734 & 0.07 & 0.0902 & 0.0005 & 2.998 & 0.0205 & 0.2446 & 0.00185 & 0.70607 & 1406 \\
\hline Kiowa_1635_13.FIN2 & 218 & 0.59 & $0.0 \leqq$ & 0.00 & 3.149 & 0.0225 & 0.2528 & 0.00195 & 0.72293 & 1444 \\
\hline Kiowa_163 & 250 & 0.56 & & 0.00 & 2.6 & 0.0 & 0.2 & 0.0024 & 0.701 & 1318 \\
\hline 5_89.F & 166.2 & 0.50 & 0.0 & & 2.5 & 0.0 & & & 1557 & 1277 \\
\hline Kiowa_1635_132.F & 156.1 & 0.40 & 0.0 & & 3.18 & 0.022 & & & 8234 & 1452 \\
\hline Kiowa_1635_25.FII & 604 & 0.06 & 0.05 & 0.00 & 3.5 & 0. & & 0.00435 & & 1536 \\
\hline Kiowa_1635_67.FI & 74.6 & 0.53 & 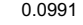 & & 3.6 & 0.0 & 0.2892 & 0.004 & 1873 & 16 \\
\hline Kiowa_1635_127.F & 82.8 & 0.46 & 0.1 & 0.00 & 3. & 0. & 0.2877 & 048 & 0.67112 & 16 \\
\hline Kiowa_1635_41.FIN2C & 214 & 0.37 & 0.1005 & 0.00075 & 3.92 & 0.037 & 0.282 & 0.0024 & 0.68207 & \\
\hline Kiowa_1635_26.FIN2 & 495 & 0.42 & 0.10191 & 0.000435 & 4.003 & 0.02 & 0.2824 & 0.00125 & 0.62424 & 1633.8 \\
\hline
\end{tabular}

\begin{tabular}{|c|c|c|c|}
\hline 0.75 & 74.4 & 0.41 & 108 \\
\hline 2.35 & 75.9 & 0.75 & 170 \\
\hline 0.95 & 77.2 & 0.55 & 34 \\
\hline 1.65 & 78.5 & 0.6 & 156 \\
\hline 1.15 & 79.1 & 0.6 & 153 \\
\hline 1.85 & 82.9 & 0.8 & 192 \\
\hline 1.2 & 84.6 & 0.6 & 157 \\
\hline 1.75 & 86.5 & 0.85 & 45 \\
\hline 1.05 & $\begin{array}{l}90.2 \\
956\end{array}$ & 0.55 & 135 \\
\hline 1.75 & $\begin{array}{r}95.6 \\
\end{array}$ & $\begin{array}{r}0.6 \\
\end{array}$ & 112 \\
\hline $\begin{array}{l}2.25 \\
2.15\end{array}$ & $\begin{array}{l}152.8 \\
158.5\end{array}$ & $\begin{array}{l}1.15 \\
1.15\end{array}$ & 298 \\
\hline $\begin{array}{l}2.15 \\
145\end{array}$ & $\begin{array}{l}158.5 \\
160.6\end{array}$ & $\begin{array}{r}1.15 \\
0.8\end{array}$ & $\begin{array}{l}440 \\
178\end{array}$ \\
\hline $\begin{array}{l}1.45 \\
1.35\end{array}$ & $\begin{array}{l}160.6 \\
163.4\end{array}$ & $\begin{array}{l}0.8 \\
0.9\end{array}$ & $\begin{array}{l}178 \\
127\end{array}$ \\
\hline 1.75 & 164.6 & 1 & $\begin{array}{l}127 \\
183\end{array}$ \\
\hline 1.25 & 166.2 & 0.85 & 234 \\
\hline 2.45 & 166.4 & 1.25 & 224 \\
\hline 3.4 & 166.7 & 1.1 & 460 \\
\hline 2.75 & 166.8 & 1.85 & 308 \\
\hline 1.9 & 167.2 & 1 & 388 \\
\hline 2.05 & 191 & 1.55 & 263 \\
\hline 2.15 & 215.6 & 1.2 & 265 \\
\hline 3 & 378.8 & 2.75 & 399 \\
\hline 2.85 & 404.2 & 1.75 & 447 \\
\hline 6 & 414 & 5 & 472 \\
\hline 2.9 & 419.1 & 2.4 & 395 \\
\hline 3 & 425.9 & 1.7 & 417 \\
\hline 3.6 & 430 & 2.2 & 435 \\
\hline 3.2 & 456.8 & 2.65 & 468 \\
\hline 4.4 & 467.6 & 2.4 & 474 \\
\hline 7.5 & 534.8 & 3.7 & 646 \\
\hline & 983.1 & 4.35 & 1015 \\
\hline 4.25 & 1001.2 & 4.7 & 1018 \\
\hline $\begin{array}{r}4.05 \\
7\end{array}$ & $\begin{array}{l}989 \\
996\end{array}$ & $\begin{array}{r}4.15 \\
7\end{array}$ & $\begin{array}{l}1023 \\
1024\end{array}$ \\
\hline $\begin{array}{l}1 \\
4.8\end{array}$ & $\begin{array}{r}996 \\
1040\end{array}$ & $\begin{array}{r}7 \\
6.5\end{array}$ & $\begin{array}{l}1024 \\
1032\end{array}$ \\
\hline & 1090 & 6 & 1039 \\
\hline 9 & 1070 & 9.5 & 1040 \\
\hline 4.5 & 1068.7 & 4.85 & 1047 \\
\hline 4.4 & 1008 & 5 & 1048 \\
\hline & 910 & & 1059 \\
\hline 5.5 & 1055 & 6.5 & 1082 \\
\hline 4.85 & 1083 & 5.5 & 1083 \\
\hline 7.5 & 1082 & 8 & 1111 \\
\hline 3.95 & 1099 & 5 & 1135 \\
\hline 3.5 & 1152.7 & 4.2 & 1140 \\
\hline & 1206 & 10 & 1164 \\
\hline 4.25 & 1133.5 & 4.5 & 1168 \\
\hline 15.5 & 1025 & 20 & 1177 \\
\hline & 1210 & 8.5 & 1179 \\
\hline 4.4 & 1150 & 6.5 & 1186 \\
\hline 6.5 & 1146 & 6.5 & 1191 \\
\hline 2.7 & 1161.6 & 3.45 & 1205 \\
\hline 9.5 & 1206 & 13.5 & 1221 \\
\hline 3.45 & 1187.2 & 4.9 & 1235 \\
\hline 6 & 1191 & 7 & 1243 \\
\hline 3.75 & 1260.6 & 4.85 & 1277 \\
\hline & 1432 & 9.5 & 1354 \\
\hline 8.5 & 1303 & 6.5 & 1363 \\
\hline 5.5 & 1350 & 7 & 1365 \\
\hline 6 & 1358 & 8 & 1366 \\
\hline & $\begin{array}{l}1400 \\
1334\end{array}$ & $\begin{array}{l}12.5 \\
105\end{array}$ & $\begin{array}{l}1404 \\
1\end{array}$ \\
\hline $\begin{array}{r}7 \\
3.25\end{array}$ & $\begin{array}{l}1334 \\
1416\end{array}$ & $\begin{array}{r}10.5 \\
5\end{array}$ & $\begin{array}{l}\text { 1407 } \\
1408\end{array}$ \\
\hline & 1409 & 9.5 & 1422 \\
\hline 4.35 & 1324 & 6 & 1422 \\
\hline 5 & 1410 & 9.5 & 1428 \\
\hline 5.5 & 1452 & 10 & 1440 \\
\hline 8.5 & 1232 & 12.5 & 1454 \\
\hline 9.5 & 1177.1. & 4.55 & 1463 \\
\hline 5.5 & 1434 & 7 & 1466 \\
\hline 14 & 1535 & 22 & 1520 \\
\hline 11.5 & 1633 & 20 & 1588 \\
\hline 12.5 & 1623 & 24 & 1634 \\
\hline 7.5 & 1601 & 12 & 1638 \\
\hline 4.1 & 1603 & 6.5 & 1657 \\
\hline
\end{tabular}

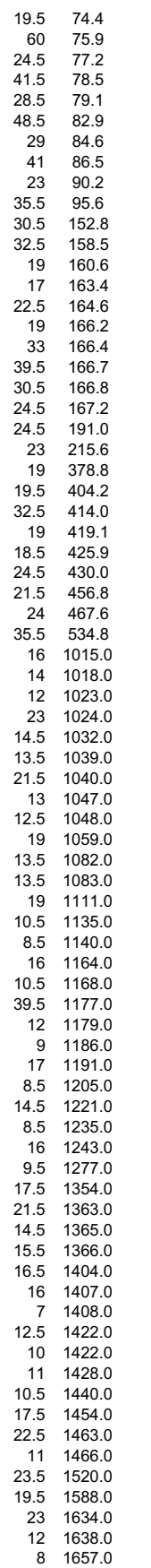

$\begin{array}{lll} & & \\ 0.4 & 1.6 & \\ 0.8 & 4.4 & \\ 0.6 & -2.3 & \\ 0.6 & 3.4 & \\ 0.6 & 3.2 & \\ 0.8 & 4.5 & \\ 0.6 & 2.9 & \\ 0.9 & -2.4 & \\ 0.6 & 1.2 & \\ 0.6 & 0.3 & \\ 1.2 & 5.6 & \\ 1.2 & 10.8 & \\ 0.8 & 1.3 & \\ 0.9 & -0.9 & \\ 1.0 & 1.6 & \\ 0.9 & 1.7 & \\ 1.3 & 2.2 & \\ 1.1 & 11.7 & \\ 1.9 & 5.8 & \\ 1.0 & 8.5 & \\ 1.6 & 3.0 & \\ 1.2 & 2.1 & \\ 2.8 & 1.3 & \\ 1.8 & 1.7 & \\ 5.0 & 2.6 & \\ 2.4 & -0.5 & \\ 1.7 & 0.4 & \\ 2.2 & -0.2 & \\ 2.7 & -0.4 & \\ 2.4 & 0.6 & \\ 3.7 & 4.2 & \\ 16.0 & 3.1 & \\ 14.0 & 1.7 & \\ 12.0 & 3.3 & \\ 23.0 & 2.7 & \\ 14.5 & -0.8 & \\ 13.5 & -4.9 & \\ 21.5 & -2.9 & \\ 13.0 & -2.1 & \\ 12.5 & 3.8 & \\ 19.0 & 14.1 & \\ 13.5 & 2.5 & \\ 13.5 & 0.0 & \\ 19.0 & 2.6 & \\ 10.5 & 3.2 & \\ 8.5 & -1.1 & \\ 16.0 & -3.6 & \\ 10.5 & 3.0 & \\ 39.5 & 12.9 & \\ 12.0 & -2.6 & \\ 9.0 & 3.0 & \\ 17.0 & 3.8 & \\ 8.5 & 3.6 & \\ 14.5 & 1.2 & \\ 8.5 & 3.9 & \\ 16.0 & 4.2 & \\ 9.5 & 1.3 & \\ 17.5 & -5.8 & \\ 21.5 & 4.4 & \\ 14.5 & 1.1 & \\ 15.5 & 0.6 & \\ 16.5 & 0.3 & \\ 16.0 & 5.2 & \\ 7.0 & -0.6 & \\ 12.5 & 0.9 & \\ 10.0 & 6.9 & \\ 11.0 & 1.3 & \\ 10.5 & -0.8 & \\ 17.5 & 15.3 & \\ 22.5 & 19.5 & \\ 11.0 & 2.2 & \\ 23.5 & -1.0 & \\ 19.5 & -2.8 & \\ 23.0 & 0.7 & \\ 12.0 & 2.3 & \\ 8.0 & 3.3 & \text { Core } \\ & & \end{array}$




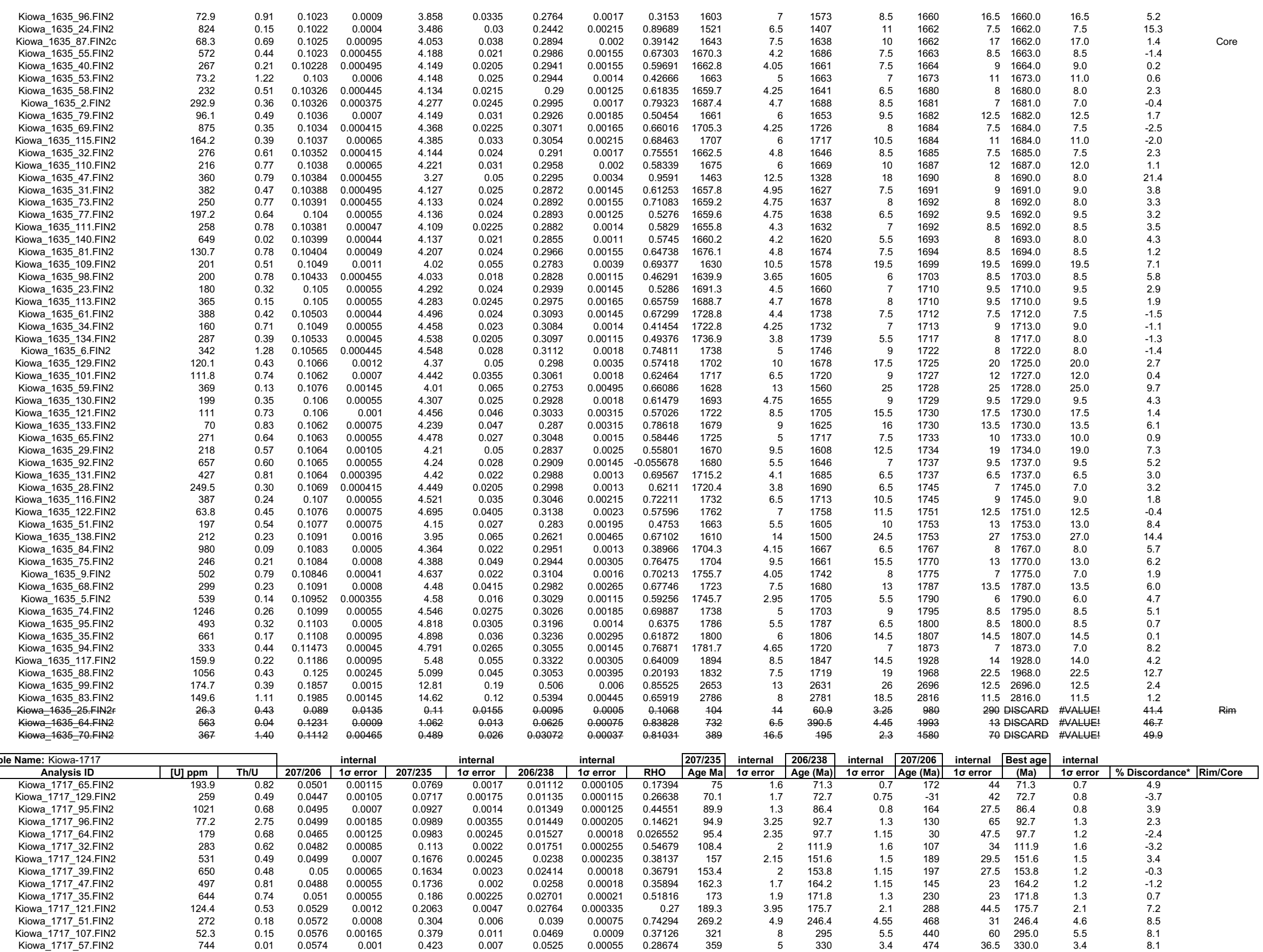




\begin{tabular}{|c|c|c|c|c|c|c|c|c|c|c|}
\hline Kiowa_1717_106.FIN2 & 602 & 0.04 & 0.0563 & 0.0011 & 0.43 & 0.0085 & 0.0541 & 0.0009 & 0.52008 & 363 \\
\hline Kiowa_1717_55.FIN2 & 114 & 0.43 & 0.0606 & 0.00095 & 0.55 & 0.0085 & 0.0651 & 0.00075 & 0.35747 & 444 \\
\hline Kiowa_1717_21.FIN2 & 298 & 0.80 & 0.0562 & 0.00055 & 0.519 & 0.006 & 0.0663 & 0.0006 & 0.53444 & 423.1 \\
\hline Kiowa_1717_131.FIN2 & 30.7 & 0.96 & 0.061 & 0.0017 & 0.584 & 0.018 & 0.0679 & 0.0011 & 0.43118 & 459 \\
\hline Kiowa_1717_109.FIN2 & 166 & 0.78 & 0.056 & 0.00075 & 0.545 & 0.007 & 0.0686 & 0.00065 & 0.39296 & 440 \\
\hline Kiowa_1717_31.FIN2 & 473 & 0.15 & 0.05638 & 0.00048 & 0.532 & 0.005 & 0.0693 & 0.00055 & 0.52152 & 432.5 \\
\hline Kiowa_1717_85.FIN2 & 510 & 0.28 & 0.0579 & 0.0005 & 0.564 & 0.005 & 0.0695 & 0.0005 & 0.40111 & 453.3 \\
\hline Kiowa_1717_123.FIN2 & 412 & 0.48 & 0.0571 & 0.00065 & 0.564 & 0.0065 & 0.0704 & 0.0007 & 0.47352 & 455.3 \\
\hline Kiowa-1717-104.FIN2 & 380 & 0.51 & 0.0551 & 0.0006 & 0.551 & 0.006 & 0.0714 & 0.0007 & 0.44269 & 445.4 \\
\hline Kiowa_1717_28.FIN2 & 52.2 & 0.48 & 0.0578 & 0.00135 & 0.584 & 0.0135 & 0.0743 & 0.00075 & 0.18449 & 464 \\
\hline Kiowa_1717_70.FIN2 & 154 & 0.27 & 0.0559 & 0.0007 & 0.596 & 0.008 & 0.0775 & 0.00055 & 0.32653 & 473.9 \\
\hline Kiowa_1717_48.FIN2 & 108.3 & 0.84 & 0.057 & 0.0007 & 0.672 & 0.008 & 0.0866 & 0.0007 & 0.38761 & 522 \\
\hline Kiowa_1717_71.FIN2 & 130.6 & 0.76 & 0.0616 & 0.00065 & 0.83 & 0.01 & 0.0972 & 0.00085 & 0.50861 & 613 \\
\hline Kiowa_1717-114.FIN2 & $\begin{array}{l}132 \\
383\end{array}$ & 0.84 & 0.0579 & 0.00095 & 0.805 & 0.015 & 0.0979 & 0.00125 & 0.50521 & 597 \\
\hline Kiowa_1717_78.FIN2 & 383 & 0.56 & 0.0602 & 0.00065 & 0.852 & 0.01 & 0.1017 & 0.0012 & $\begin{array}{r}0.5564 \\
073267\end{array}$ & 625 \\
\hline Kiowa_1717_40.FIN2 & $\begin{array}{r}123.2 \\
120\end{array}$ & 1.23 & 0.0756 & 0.00095 & 1.235 & 0.0225 & 0.1213 & 0.00215 & 0.73267 & 812 \\
\hline $\begin{array}{l}\text { Kiowa-1717_-81.FIN2 } \\
\text { Kiowa } 1717 \text { - FIN2 }\end{array}$ & $\begin{array}{r}120 \\
806\end{array}$ & $\begin{array}{l}1.04 \\
258\end{array}$ & 0.0764 & $\begin{array}{l}0.00125 \\
0.00095\end{array}$ & $\begin{array}{l}1.344 \\
1529\end{array}$ & 0.0265 & 0.1257 & 0.00215 & 0.61228 & 858 \\
\hline $\begin{array}{l}\text { Kiowa_ } 17177 \text { - } 45 \text {.FIN2 } \\
\text { Kiowa } 1717 \\
135 \text {.FIN2 }\end{array}$ & $\begin{array}{l}80.6 \\
33.6\end{array}$ & $\begin{array}{l}2.58 \\
2.04\end{array}$ & $\begin{array}{l}0.0811 \\
0.0685\end{array}$ & $\begin{array}{r}0.00095 \\
0.0009\end{array}$ & $\begin{array}{l}1.529 \\
1.456\end{array}$ & $\begin{array}{l}0.0215 \\
0.0205\end{array}$ & $\begin{array}{l}0.1395 \\
0.1519\end{array}$ & $\begin{array}{l}0.00175 \\
0.001\end{array}$ & $\begin{array}{l}0.62383 \\
0.35175\end{array}$ & $\begin{array}{l}937 \\
907\end{array}$ \\
\hline Kiowa_1717_24.FIN2 & 93.1 & 0.47 & 0.0718 & 0.001 & 1.612 & 0.0255 & 0.1636 & 0.0024 & 0.58564 & 968 \\
\hline Kiowa_1717_74.FIN2 & 18.62 & 1.98 & 0.0727 & 0.0014 & 1.651 & 0.034 & 0.1625 & 0.00215 & 0.35754 & 977 \\
\hline Kiowa_-1717_-72.FIN2 & 97 & 0.44 & 0.0726 & 0.00085 & 1.791 & 0.023 & 0.1779 & 0.00175 & 0.46265 & 1039 \\
\hline Kiowa_1717_117.FIN2 & 262 & 0.26 & 0.0733 & 0.00095 & 1.512 & 0.041 & 0.1463 & 0.0039 & 0.86808 & 915 \\
\hline Kiowa_1717_60.FIN2 & 122 & 0.48 & 0.0727 & 0.00065 & 1.67 & 0.0155 & 0.1634 & 0.0012 & 0.49455 & 995 \\
\hline Kiowa_1717_13.FIN2 & 22.1 & 2.52 & 0.0746 & 0.00145 & 1.866 & 0.037 & 0.1777 & 0.0017 & 0.24568 & 1056 \\
\hline Kiowa_1717_103.FIN2 & 51.9 & 0.89 & 0.0741 & 0.0012 & 1.73 & 0.0325 & 0.1687 & 0.00255 & 0.62541 & 1019 \\
\hline Kiowa_1717_38.FIN2 & 83 & 0.22 & 0.0734 & 0.001 & 1.515 & 0.0265 & 0.1548 & 0.00255 & 0.68089 & 933 \\
\hline Kiowa_1717_77.FIN2 & 67 & 0.67 & 0.0734 & 0.00075 & 1.776 & 0.02 & 0.1718 & 0.00145 & 0.51337 & 1035 \\
\hline Kiowa_1717_66.FIN2 & 69.9 & 0.80 & 0.0737 & 0.00075 & 1.678 & 0.0175 & 0.1635 & 0.00135 & 0.40832 & 998 \\
\hline Kiowa_1717_50.FIN2 & 72.9 & 1.18 & 0.0739 & 0.0007 & 1.727 & 0.018 & 0.1694 & 0.00105 & 0.40415 & 1015 \\
\hline Kiowa_1717_99.FIN2 & 109.2 & 0.99 & 0.0741 & 0.0008 & 1.578 & 0.0225 & 0.1534 & 0.00215 & 0.70297 & 955 \\
\hline Kiowa_1717_44.FIN2 & 81.2 & 0.29 & 0.0742 & 0.0007 & 1.849 & 0.0185 & 0.1842 & 0.0013 & 0.40543 & 1060 \\
\hline Kiowa_1717_75.FIN2 & 364 & 0.23 & 0.0748 & 0.00085 & 1.801 & 0.0235 & 0.1747 & 0.0023 & 0.60542 & 1040 \\
\hline Kiowa_1717_22.FIN2 & 391 & 0.40 & 0.075 & 0.0011 & 1.827 & & 0.1763 & 0.00355 & 0.71482 & 1041 \\
\hline Kiowa_1717_93.FIN2 & 133 & 0.52 & 0.0742 & 0.0007 & 1.801 & 0.026 & 0.1752 & 0.00205 & 0.73694 & 1039 \\
\hline Kiowa_1717_54.FIN2 & 588 & 0.23 & 0.07436 & 0.000385 & 1.75 & 0.013 & 0.1684 & 0.00115 & 0.75573 & 1025.6 \\
\hline Kiowa_1717_79.FIN2 & 47.4 & 0.98 & 0.0749 & 0.0009 & 1.821 & 0.0225 & 0.1734 & 0.0015 & 0.42187 & 1052 \\
\hline Kiowa_1717_119.FIN2 & 146 & 0.83 & 0.0749 & 0.00085 & 1.589 & 0.023 & 0.15 & 0.002 & 0.69521 & 960 \\
\hline Kiowa_1717_87.FIN2 & 174 & 0.96 & 0.075 & 0.0006 & 1.821 & 0.015 & 0.1743 & 0.00115 & 0.47485 & 1051 \\
\hline Kiowa_1717_53.FIN2 & 133 & 0.42 & 0.0751 & 0.0006 & 1.865 & 0.0155 & 0.1778 & 0.0012 & 0.43007 & 1066 \\
\hline Kiowa_1717_88.FIN2 & 246 & 0.66 & $\begin{array}{r}0.075 \\
0.0753\end{array}$ & 0.0005 & 1.905 & 0.0155 & 0.1828 & 0.00135 & 0.64063 & 1081 \\
\hline Kiowa_1717_126.FIN2 & $\begin{array}{l}210 \\
365\end{array}$ & 0.59 & 0.0753 & 0.00085 & 1.575 & 0.0195 & $\begin{array}{r}0.15 \\
01618\end{array}$ & $\begin{array}{r}0.0021 \\
0\end{array}$ & $\begin{array}{r}0.7059 \\
0.51852\end{array}$ & $\begin{array}{r}956 \\
1018\end{array}$ \\
\hline Kiowa_1717_105.FIN2 & 365 & 0.09 & 0.0751 & 0.0006 & 1.728 & 0.014 & 0.1618 & 0.00115 & 0.51852 & 1018 \\
\hline $\begin{array}{l}\text { Kiowa_- } 1717 \text { - } 25 \text {.FIN2 } \\
\text { Kiowa } 177 \text { BFIN2 }\end{array}$ & $\begin{array}{r}178 \\
472\end{array}$ & 0.86 & 0.0755 & $\begin{array}{r}0.0009 \\
0.00095\end{array}$ & $\begin{array}{l}1.862 \\
1854\end{array}$ & 0.0235 & 0.1802 & $\begin{array}{r}0.0022 \\
0.00145\end{array}$ & 0.53699 & 1063 \\
\hline 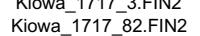 & $\begin{array}{l}47.2 \\
38.7\end{array}$ & $\begin{array}{l}1.76 \\
0.59\end{array}$ & $\begin{array}{l}0.066 \\
0.071\end{array}$ & $\begin{array}{l}0.00095 \\
0.00135\end{array}$ & $\begin{array}{l}1.854 \\
1.865\end{array}$ & $\begin{array}{r}0.0225 \\
0.032\end{array}$ & $\begin{array}{l}0.1773 \\
0.1765\end{array}$ & $\begin{array}{l}0.00145 \\
0.00265\end{array}$ & $\begin{array}{l}0.31818 \\
0.49523\end{array}$ & $\begin{array}{l}1060 \\
1062\end{array}$ \\
\hline Kiowa_1717_8.FIN2 & 235.4 & 0.45 & 0.07497 & 0.000385 & 1.864 & 0.0105 & 0.1785 & 0.00085 & 0.49409 & 1068.3 \\
\hline Kiowa_1717-12.FIN2 & 42.5 & 1.18 & 0.076 & 0.0009 & 1.831 & 0.0215 & 0.1724 & 0.00105 & 0.22446 & 1052 \\
\hline Kiowa_1717_118.FIN2 & 430 & 0.42 & 0.0756 & 0.00065 & 1.862 & 0.0175 & 0.1736 & 0.00165 & 0.5534 & 1066 \\
\hline Kiowa_1717_1.FIN2 & 468 & 0.39 & 0.07535 & 0.00048 & 1.838 & 0.0165 & 0.176 & 0.00135 & 0.71921 & 1056 \\
\hline Kiowa_1717_89.FIN2 & 106.8 & 1.16 & 0.0756 & 0.0006 & 1.768 & 0.015 & 0.1682 & 0.0011 & 0.41288 & 1032 \\
\hline Kiowa_1717_110.FIN2 & 27.3 & 0.55 & 0.0774 & 0.0014 & 2.005 & 0.0365 & 0.1825 & 0.0023 & 0.34946 & 1109 \\
\hline Kiowa_1717_113.FIN2 & 272 & 1.03 & 0.0752 & 0.00055 & 1.872 & 0.0165 & 0.174 & 0.0014 & 0.24592 & 1069 \\
\hline Kiowa_1717_61.FIN2 & 304 & 0.54 & 0.07521 & 0.00042 & 1.784 & 0.0125 & 0.1679 & 0.00095 & 0.63702 & 1039.3 \\
\hline Kiowa_1717_43.FIN2 & 222.2 & 0.44 & 0.0755 & 0.0005 & 1.854 & 0.0115 & 0.1813 & 0.0011 & 0.43567 & 1064.6 \\
\hline Kiowa_1717_68.FIN2 & 138 & 0.45 & 0.0776 & 0.00145 & 1.872 & 0.0 & 0.177 & 0.0035 & 0.61055 & 1058 \\
\hline Kiowa_1717_137.FIN2 & 187 & 0.58 & 0.0761 & & 1.853 & 0.0 & & 0.0022 & 2781 & 1062 \\
\hline Kiowa_1717_69.FII & 58.6 & 1.12 & & & & & & & & 1056 \\
\hline Kiowa_1717_52.FI & 119.6 & 1.25 & 0.076 & & 1.879 & 0.0 & & 0.00145 & & 1071 \\
\hline Kiowa_17 & 107.3 & 0.83 & 0.07597 & 0.000 & 1.829 & & & 0.00085 & & 1054 \\
\hline Kiowa_1717_116.FIN2 & 38.9 & 0.76 & 0.0765 & 0.00 & 1.91 & 0.03 & & 0.0021 & 3659 & 1078 \\
\hline Kiowa_1717_59.FIN2 & 116.4 & 0.89 & & 0.00 & 1.741 & 0.0 & & 0.0017 & 0.71278 & 1020 \\
\hline Kiowa_1717_73.FIN2 & 171 & 0.42 & 0.07 & 0.00 & 1.545 & 0.0 & & 0.00285 & & 945 \\
\hline Kiowa_1717_76.FIN2 & 142 & 2.33 & 0.0765 & 0.000 & 1.856 & 0.02 & 0.1743 & 0.00165 & 0.52847 & 1061 \\
\hline Kiowa_1717_56.FIN2 & 237 & 0.52 & 0.0769 & 0.00055 & 1.938 & 0.0 & 0.1787 & 0.0011 & 0.50409 & 1092 \\
\hline Kiowa_1717_136.FIN2 & 410 & 0.60 & 0.0771 & 0.00055 & 1.845 & 0.01 & 0.173 & 0.0013 & 0.57207 & 1059 \\
\hline Kiowa_1717_138.FIN2 & 93 & 0.78 & 0.0772 & 0.0007 & 1.872 & 0.016 & 0.1772 & 0.0011 & & 1069 \\
\hline Kiowa_1717_-98.FIN2 & & 0.31 & & 0.0006 & 1.86 & 0.0155 & 0.1729 & 0.00105 & 0.45196 & 1065 \\
\hline $\begin{array}{l}\text { Kiowa_-1717_-46.FIN2 } \\
\text { Kowa 1717 20.FIN2 }\end{array}$ & $\begin{array}{r}110.1 \\
158\end{array}$ & $\begin{array}{l}0.76 \\
0.82\end{array}$ & $\begin{array}{l}0.0783 \\
0.079\end{array}$ & $\begin{array}{r}0.00115 \\
0.0007\end{array}$ & $\begin{array}{l}1.933 \\
1703\end{array}$ & $\begin{array}{r}0.028 \\
0.0225\end{array}$ & 0.1828 & $\begin{array}{r}0.0024 \\
0.00165\end{array}$ & $\begin{array}{l}0.37205 \\
0.74111\end{array}$ & 1090 \\
\hline $\begin{array}{l}\text { Liowa- } 1 / 17 \text {-20.FIN2 } \\
\text { Kiowa } 1717 \text { 63.FIN2 }\end{array}$ & $\begin{array}{l}158 \\
106.8\end{array}$ & $\begin{array}{l}0.82 \\
0.73\end{array}$ & $\begin{array}{l}0.0781 \\
0.0781\end{array}$ & 0.00075 & $\begin{array}{l}1.173 \\
1.783\end{array}$ & $\begin{array}{r}0.0225 \\
0.016\end{array}$ & $\begin{array}{l}0.1552 \\
0.1634\end{array}$ & $\begin{array}{l}0.00165 \\
0.0012\end{array}$ & 0.14111 & 1004 \\
\hline Kiowa-1717-62.FIN2 & $\begin{array}{l}140.0 \\
141.1\end{array}$ & 0.66 & 0.0782 & 0.00095 & 2.153 & 0.028 & $\begin{array}{l}0.1034 \\
0.1965\end{array}$ & 0.002 & $\begin{array}{r}0.020 \\
0.42095\end{array}$ & 1164 \\
\hline Kiowa_1717_29.FIN2 & 115.2 & 0.44 & 0.0784 & 0.0006 & 2.053 & 0.0175 & 0.1922 & 0.00135 & 0.55466 & 1131 \\
\hline Kiowa_1717_122.FIN2 & 304 & 0.63 & 0.078 & 0.00055 & 1.826 & 0.015 & 0.166 & 0.0013 & 0.63825 & 1053 \\
\hline Kiowa_1717_91.FIN2 & 57.6 & 2.69 & 0.0796 & 0.0011 & 1.969 & 0.033 & 0.1777 & 0.0019 & 0.54229 & 1098 \\
\hline Kiowa_1717_97.FIN2 & 169.9 & 1.03 & 0.0797 & 0.001 & 1.582 & 0.02 & 0.143 & 0.002 & 0.5454 & 964 \\
\hline
\end{tabular}

\begin{tabular}{|c|c|c|c|}
\hline 6.5 & 340 & 5.5 & 426 \\
\hline 5.5 & 406.6 & 4.6 & 585 \\
\hline 3.9 & 413.8 & 3.75 & 446 \\
\hline 11.5 & 423 & 6.5 & 560 \\
\hline 4.75 & 427.5 & & \\
\hline 3.4 & 432 & 3.35 & 451 \\
\hline 3.3 & 433.2 & 3.05 & 512 \\
\hline 4.25 & 438.5 & & 482 \\
\hline 3.85 & 444.2 & 4.2 & 394 \\
\hline 8.5 & 461.6 & 4.6 & 467 \\
\hline 4.85 & 480.9 & 3.35 & 419 \\
\hline 5 & 535.3 & 4.1 & 470 \\
\hline & 597.8 & 4.9 & 644 \\
\hline 8.5 & 602 & 7.5 & 487 \\
\hline 5.5 & 624 & & 590 \\
\hline 10.5 & 737 & 12.5 & 1067 \\
\hline 11.5 & 762 & 12 & 1063 \\
\hline 8.5 & 841 & 10 & 1203 \\
\hline 8.5 & 911 & 5.5 & 859 \\
\hline 10 & 975 & 13 & 939 \\
\hline 15 & 969 & 12 & 949 \\
\hline 8.5 & 1004 & $\begin{array}{l}9.5 \\
.02\end{array}$ & 987 \\
\hline 10 & $\begin{array}{l}014 \\
075\end{array}$ & 22 & 987 \\
\hline . & 170 & 0.0 & 989 \\
\hline 13 & $1054^{2}$ & (5.5 & 990 \\
\hline 11.5 & 925 & $\begin{array}{l}14 \\
14\end{array}$ & 994 \\
\hline & 1021 & 8 & 1016 \\
\hline 7 & 975 & 7.5 & 1017 \\
\hline 6.5 & 1008 & 6 & 1019 \\
\hline & 918 & 12 & 1025 \\
\hline 6.5 & 1089 & 7 & 1033 \\
\hline 8.5 & 1036 & 12.5 & 1040 \\
\hline 13.5 & 1042 & 19.5 & 1041 \\
\hline 9.5 & 1039 & 11 & 1041 \\
\hline 4.8 & 1003 & 6.5 & 1048 \\
\hline 8 & 1030 & 8 & 1050 \\
\hline 9 & 900 & 11.5 & 1052 \\
\hline 5.5 & 1035 & 6.5 & 1056 \\
\hline 5.5 & 1054 & 6.5 & 1058 \\
\hline 5.5 & 1082 & & 1058 \\
\hline 7.5 & 899 & 11.5 & 1058 \\
\hline 5.5 & 966 & 6.5 & 1059 \\
\hline 8.5 & 1067 & 12 & 1063 \\
\hline 115 & $\begin{array}{l}1051 \\
104\end{array}$ & $\begin{array}{r}8 \\
15\end{array}$ & $\begin{array}{l}1064 \\
104\end{array}$ \\
\hline 3.55 & 10583 & 4.55 & 1065 \\
\hline 7.5 & 1025 & 5.5 & 1066 \\
\hline 6.5 & 1031 & 9 & 1069 \\
\hline & 1045 & 7.5 & 1070 \\
\hline 5.5 & 1002 & 6 & 1070 \\
\hline 12.5 & 1079 & 12.5 & 1070 \\
\hline & 1036 & 8 & 1070 \\
\hline 4.55 & 1000 & 5.5 & 1071 \\
\hline 4.25 & 1074 & 6 & 1074 \\
\hline 13.5 & 1047 & 19 & 1074 \\
\hline 7.5 & 1060 & 12 & 1076 \\
\hline 9.5 & 1033 & 9 & 1078 \\
\hline & 1056 & 8 & 1085 \\
\hline 4.95 & 1024.9 & 4.65 & 1086 \\
\hline 11 & 1041 & 11.5 & 1087 \\
\hline 7.5 & 966 & 9.5 & 1092 \\
\hline 11.5 & 873 & 16 & 1102 \\
\hline 7.5 & 1035 & 9 & 1104 \\
\hline 5.5 & 1059 & 6 & 1109 \\
\hline 6 & 1028 & 7 & 1115 \\
\hline & 1051 & 6 & 1115 \\
\hline 5.5 & 1028 & 6 & 1116 \\
\hline 10 & 1080 & 13 & 1126 \\
\hline 8.5 & 92 & 9 & 133 \\
\hline & & 6.5 & \\
\hline 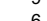 & 1150 & 10.5 & \\
\hline 55 & 1133 & 75 & \\
\hline 11.5 & 1053 & 10.5 & $\begin{array}{l}1140 \\
1156\end{array}$ \\
\hline 7.5 & 861 & 11.5 & 1175 \\
\hline
\end{tabular}

\begin{tabular}{|c|c|c|c|}
\hline 40.5 & 340.0 & 5.5 & 6.3 \\
\hline 33.5 & 406.6 & 4.6 & \\
\hline & 413.8 & 3.8 & \\
\hline 60 & 423.0 & 6.5 & \\
\hline 28 & 427.5 & 4.0 & \\
\hline 19 & 432.0 & 3.4 & \\
\hline 20 & 433.2 & 3.1 & \\
\hline & 438.5 & 4.1 & 3.7 \\
\hline 23.5 & 444.2 & 4.2 & \\
\hline 49.5 & 461.6 & 4.6 & \\
\hline & 480.9 & 3.4 & \\
\hline 25.5 & 535.3 & 4.1 & \\
\hline & 597.8 & 4.9 & \\
\hline 34.5 & 602.0 & 7.5 & \\
\hline 24.5 & 624.0 & 7.0 & \\
\hline 27 & 737.0 & 12.5 & \\
\hline 33 & 762.0 & 12.0 & \\
\hline $\begin{array}{l}22.5 \\
285\end{array}$ & 841.0 & 10.0 & \\
\hline $\begin{array}{r}28.5 \\
29\end{array}$ & 859.0 & 28.5 & \\
\hline $\begin{array}{r}29 \\
415\end{array}$ & 939.0 & $\begin{array}{l}29.0 \\
415\end{array}$ & \\
\hline $\begin{array}{l}41.5 \\
24.5\end{array}$ & $\begin{array}{r}949.0 \\
987.0\end{array}$ & $\begin{array}{l}41.5 \\
245\end{array}$ & $\begin{array}{l}-2.1 \\
-6.8\end{array}$ \\
\hline 26.5 & 987.0 & 26.5 & 11.4 \\
\hline 18 & 989.0 & 18.0 & 1.4 \\
\hline 41 & 990.0 & 41.0 & -6.5 \\
\hline 34 & 991.0 & 34.0 & -1.2 \\
\hline 28.5 & 994.0 & 28.5 & \\
\hline 20.5 & 1016.0 & 20.5 & -0.5 \\
\hline 20.5 & 1017.0 & 20.5 & \\
\hline & 1019.0 & 20.0 & \\
\hline 22.5 & 1025.0 & 22.5 & \\
\hline 19 & 1033.0 & 19.0 & \\
\hline 24.5 & 1040.0 & 24.5 & 0.4 \\
\hline 29.5 & 1041.0 & 29.5 & -0.1 \\
\hline 18 & 1041.0 & 18.0 & 0.2 \\
\hline 10.5 & 1048.0 & 10.5 & 4.3 \\
\hline 24 & 1050.0 & 24.0 & 1.9 \\
\hline 23 & 1052.0 & 23.0 & 14.4 \\
\hline 16 & 1056.0 & 16.0 & 2.0 \\
\hline 16 & 1058.0 & 16.0 & 0.4 \\
\hline 13.5 & 1058.0 & 13.5 & -2.3 \\
\hline 22 & 1058.0 & 22.0 & 15.0 \\
\hline 16 & 1059.0 & 16.0 & 8.8 \\
\hline 24.5 & 1063.0 & 24.5 & -0.4 \\
\hline 25 & 1064.0 & 25.0 & 1.2 \\
\hline $\begin{array}{c}35.5 \\
10\end{array}$ & 1064.0 & 35.5 & 1.4 \\
\hline & 1065.0 & 10.0 & 0.6 \\
\hline $\begin{array}{r}24.5 \\
18\end{array}$ & 1066.0 & $\begin{array}{l}24.5 \\
180\end{array}$ & 3.8 \\
\hline $\begin{array}{l}18 \\
13\end{array}$ & $\begin{array}{l}1069.0 \\
1070 .\end{array}$ & $\begin{array}{l}18.0 \\
130\end{array}$ & $\begin{array}{l}3.6 \\
2.6\end{array}$ \\
\hline 17 & 1070.0 & $\begin{array}{l}10.0 \\
17.0\end{array}$ & \\
\hline 39.5 & 1070.0 & 39.5 & -0.8 \\
\hline 14 & 1070.0 & 14.0 & \\
\hline 11.5 & 1071.0 & 11.5 & \\
\hline 13.5 & 1074.0 & 13.5 & \\
\hline 36.5 & 1074.0 & 36.5 & \\
\hline 20.5 & 1076.0 & 20.5 & \\
\hline & 1078.0 & 24.0 & 4.2 \\
\hline 17.5 & 1085.0 & 17.5 & \\
\hline 13.5 & 1086.0 & 13.5 & \\
\hline 29.5 & 1087.0 & 29.5 & \\
\hline 16.5 & 1092.0 & 16.5 & \\
\hline 32.5 & 1102.0 & 32.5 & \\
\hline 19.5 & 1104.0 & 19.5 & \\
\hline 15 & 1109.0 & 15.0 & \\
\hline 14 & 1115.0 & 14.0 & \\
\hline 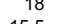 & 1115.0 & 18.0 & \\
\hline 15.5 & 1116.0 & 15.5 & \\
\hline $\begin{array}{l}30.5 \\
305\end{array}$ & 1126.0 & 30.5 & \\
\hline 18.5 & 1733.0 & 18.5 & \\
\hline 255 & 7136.0 & 19.0 & \\
\hline $\begin{array}{l}20.5 \\
155\end{array}$ & 1139.0 & 25.5 & \\
\hline 150.0 & 1146.0 & 15.5 & \\
\hline & 1146.0 & 14.0 & \\
\hline 20.5 & 150.0 & 20.5 & \\
\hline & 1175.0 & 25.0 & \\
\hline
\end{tabular}




\begin{tabular}{|c|c|c|c|c|c|c|c|c|c|c|c|c|c|c|c|c|c|c|c|}
\hline Kiowa_1717_133.FIN2 & 51 & 0.43 & 0.0816 & 0.0013 & 2.578 & 0.046 & 0.2271 & 0.00325 & 0.51709 & 1282 & 13 & 1320 & 17.5 & 1195 & 31.5 & 1195.0 & 31.5 & -10.5 & \\
\hline Kiowa_1717_130.FIN2 & 95 & 0.34 & 0.0828 & 0.001 & 2.522 & 0.0305 & 0.2186 & 0.0026 & 0.54797 & 1275 & 9 & 1272 & 13.5 & 1242 & 24 & 1242.0 & 24.0 & -2.4 & \\
\hline Kiowa_-1717_14.FIN2 & 242 & 0.42 & 0.083 & 0.00055 & 2.327 & 0.016 & 0.2009 & 0.0015 & 0.55455 & 1218.8 & 4.85 & 1179 & 8 & 1260 & 13.5 & 1260.0 & 13.5 & 6.4 & \\
\hline Kiowa_1717_128.FIN2 & 84.9 & 1.05 & 0.0838 & 0.00115 & 2.08 & 0.0325 & 0.1769 & 0.00245 & 0.51096 & 1134 & 10.5 & 1048 & 13.5 & 1264 & 27.5 & 1264.0 & 27.5 & 17.1 & \\
\hline Kiowā_1717_2.FIN2 & 227 & 0.56 & 0.0848 & 0.0006 & 2.195 & 0.02 & 0.1871 & 0.0017 & 0.70022 & 1177 & 6.5 & 1105 & 9 & 1310 & 13.5 & 1310.0 & 13.5 & 15.6 & \\
\hline Kiowa_1717_141.FIN2 & 144 & 1.19 & 0.0866 & 0.0005 & 2.669 & 0.0195 & 0.2267 & 0.00155 & 0.66165 & 1318 & 5.5 & 1316 & 8 & 1344 & 11.5 & 1344.0 & 11.5 & 2.1 & \\
\hline Kiowa_1717_90.FIN2 & 702 & 0.32 & 0.0871 & 0.00065 & 2.544 & 0.0305 & 0.2109 & 0.00275 & 0.81263 & 1280 & 8.5 & 1231 & 14.5 & 1354 & 15 & 1354.0 & 15.0 & 9.1 & \\
\hline Kiowa_1717_132.FIN2 & 50.9 & 0.22 & 0.0904 & 0.0012 & 3.029 & 0.04 & 0.2421 & 0.0028 & 0.46563 & 1408 & 10 & 1395 & 14.5 & 1403 & 25 & 1403.0 & 25.0 & 0.6 & \\
\hline Kiowa__1717_37.FIN2 & 140 & 0.50 & 0.0902 & 0.0005 & 3.207 & 0.019 & 0.2634 & 0.0014 & 0.52994 & 1457.3 & 4.6 & 1507 & 7 & 1425 & 10.5 & 1425.0 & 10.5 & -5.8 & \\
\hline Kiowa_1717_49.FIN2 & 561 & 0.57 & 0.0947 & 0.00075 & 3.049 & 0.027 & 0.2335 & 0.0015 & 0.50254 & 1417 & 7 & 1352 & 8 & 1518 & 14.5 & 1518.0 & 14.5 & 10.9 & \\
\hline Kiowa_1717-139.FIN2 & 229 & 0.28 & 0.0958 & 0.00105 & 3.543 & 0.045 & 0.2727 & 0.0033 & 0.5983 & 1529 & 10 & 1551 & 16.5 & 1522 & 21.5 & 1522.0 & 21.5 & -1.9 & \\
\hline Kiowa_1717_41.FIN2 & 138 & 1.18 & $\begin{array}{r}0.0973 \\
0.0789\end{array}$ & 0.0007 & 3.988 & 0.037 & 0.3042 & 0.0027 & 0.69784 & 1628 & 7.5 & 1710 & 13.5 & 1564 & 13.5 & 1564.0 & 13.5 & -9.3 & \\
\hline Kiowa_1717_83.FIN2 & 727 & 0.34 & 0.09788 & 0.000475 & 3.716 & 0.018 & 0.2702 & 0.00145 & 0.61983 & 1573.6 & 3.95 & 1541 & 7.5 & 1585 & 8.5 & 1585.0 & 8.5 & 2.8 & \\
\hline Kiowa_1717_80.FIN2 & $\begin{array}{r}248 \\
1735\end{array}$ & 0.16 & 0.0997 & 0.001 & $\begin{array}{r}3.27 \\
3695\end{array}$ & 0.041 & 0.2367 & 0.003 & 0.68112 & 1469 & 10 & 1366 & 15.5 & 1604 & 19 & 1604.0 & 19.0 & 14.8 & \\
\hline $\begin{array}{l}\text { Kiowa_1717 } 67 \text {-FIN2 } 2 \\
\text { Kiowa } 1717 \text { 1115.FIN2 }\end{array}$ & $\begin{array}{r}173.5 \\
260\end{array}$ & 0.58 & $\begin{array}{l}0.1001 \\
0.1008\end{array}$ & $\begin{array}{l}0.00095 \\
0.00135\end{array}$ & $\begin{array}{r}3.695 \\
3.4\end{array}$ & $\begin{array}{l}0.038 \\
0.055\end{array}$ & $\begin{array}{l}0.2662 \\
0.2404\end{array}$ & $\begin{array}{r}0.0026 \\
0.00325\end{array}$ & 0.59504 & 1569 & 8 & 1520 & 13 & 1614 & 17 & 1614.0 & 17.0 & 5.8 & \\
\hline $\begin{array}{l}\text { Kiowa_ } 1717 \text { - } 115 \text {.FIN2 } \\
\text { Kiowa } 1717 \text { 120.FIN2r }\end{array}$ & $\begin{array}{r}260 \\
184.4\end{array}$ & $\begin{array}{l}0.16 \\
0.49\end{array}$ & $\begin{array}{l}0.1008 \\
0.1002\end{array}$ & $\begin{array}{l}0.00135 \\
0.0013\end{array}$ & $\begin{array}{r}3.41 \\
3.842\end{array}$ & $\begin{array}{l}0.055 \\
0.043\end{array}$ & $\begin{array}{l}0.2404 \\
0.2729\end{array}$ & $\begin{array}{r}0.00325 \\
0.004\end{array}$ & $\begin{array}{l}0.62944 \\
0.53453\end{array}$ & $\begin{array}{l}1505 \\
1600\end{array}$ & $\begin{array}{r}13 \\
9.5\end{array}$ & $\begin{array}{l}1386 \\
1553\end{array}$ & $\begin{array}{r}16.5 \\
20\end{array}$ & $\begin{array}{l}1614 \\
1617\end{array}$ & $\begin{array}{l}23.5 \\
24\end{array}$ & $\begin{array}{l}1614.0 \\
16170\end{array}$ & $\begin{array}{l}23.5 \\
24.0\end{array}$ & $\begin{array}{l}14.1 \\
4.0\end{array}$ & Bim \\
\hline Kiowa_1717_11.FIN2 & 105.1 & 0.46 & 0.1011 & 0.001 & 3.766 & 0.037 & 0.2686 & 0.00195 & 0.43647 & 1582 & 7.5 & 1533 & 10 & 1631 & 17.5 & 1631.0 & 17.5 & 6.0 & №n $25+2$ \\
\hline Kiowa_1717_33.FIN2 & 378 & 0.05 & 0.1011 & 0.0006 & 4.206 & 0.032 & 0.3058 & 0.0021 & 0.68206 & 1672 & 6.5 & 1719 & 10.5 & 1638 & 11 & 1638.0 & 11.0 & -4.9 & \\
\hline Kiowa_1717_18.FIN2 & 29.1 & 1.12 & 0.1018 & 0.001 & 3.886 & 0.0385 & 0.2724 & 0.002 & 0.53626 & 1608 & 8 & 1552 & 10 & 1641 & 17.5 & 1641.0 & 17.5 & 5.4 & \\
\hline Kiowa_1717_17.FIN2 & 90.2 & 1.29 & 0.1014 & 0.0006 & 4.165 & 0.027 & 0.2934 & 0.00195 & 0.53215 & 1665 & 5.5 & 1657 & 9.5 & 1644 & 11 & 1644.0 & 11.0 & -0.8 & \\
\hline Kiowa_1717_36.FIN2 & 107.1 & 0.82 & 0.1017 & 0.001 & 4.021 & 0.0485 & 0.2927 & 0.0036 & 0.63475 & 1634 & 9.5 & 1651 & 18 & 1648 & 19.5 & 1648.0 & 19.5 & -0.2 & \\
\hline Kiowa_1717_58.FIN2 & 54.6 & 0.41 & 0.1022 & 0.0009 & 3.95 & 0.05 & 0.2729 & 0.0023 & 0.71112 & 1617 & 10.5 & 1557 & 12 & 1652 & 17 & 1652.0 & 17.0 & 5.8 & \\
\hline Kiowa_1717_42.FIN2 & 224 & 0.21 & 0.103 & 0.00105 & 3.394 & 0.043 & 0.2446 & 0.0033 & 0.69159 & 1500 & 10 & 1407 & 17 & 1665 & 19 & 1665.0 & 19.0 & 15.5 & \\
\hline Kiowa_1717_101.FIN2 & 158 & 0.76 & 0.1041 & 0.0011 & 4.26 & 0.0475 & 0.2939 & 0.00325 & 0.58018 & 1682 & 9 & 1658 & 16.5 & 1684 & 19 & 1684.0 & 19.0 & 1.5 & \\
\hline Kiowa-_1717_19.FIN2 & 766 & 0.22 & 0.104 & 0.0005 & 3.653 & 0.035 & 0.2508 & 0.0022 & 0.85114 & 1559 & 7.5 & 1441 & 11.5 & 1692 & 9 & 1692.0 & 9.0 & 14.8 & \\
\hline Kiowa_1717_134.FIN2 & 351 & 0.48 & 0.1046 & 0.0005 & 4.297 & 0.025 & 0.2959 & 0.00155 & 0.66607 & 1693.6 & 4.75 & 1670 & 7.5 & 1704 & 9 & 1704.0 & 9.0 & 2.0 & \\
\hline Kiowa_1717_102.FIN2 & 178 & 0.29 & 0.1058 & 0.0011 & 3.871 & 0.048 & 0.2602 & 0.00365 & 0.61013 & 1600 & 10 & 1487 & 18.5 & 1715 & 20 & 1715.0 & 20.0 & 13.3 & \\
\hline Kiowa_-1717_27.FIN2 & 203 & 0.30 & 0.1075 & 0.0014 & 3.53 & 0.065 & 0.2414 & 0.00415 & 0.73919 & 1519 & 15.5 & 1388 & 21.5 & 1733 & 24 & . 1733.0 & 24.0 & 19.9 & \\
\hline Kiowa_1717_23.FIN2 & 253 & 0.60 & 0.1068 & 0.0006 & 4.657 & 0.0385 & 0.3143 & 0.00235 & 0.73291 & 1757 & 7 & 1760 & 11.5 & 1740 & 10.5 & 1740.0 & 10.5 & -1.1 & \\
\hline Kiowa_1717_94.FIN2 & 79.5 & 1.17 & 0.1093 & 0.00155 & 4.92 & 0.09 & 0.33 & 0.006 & 0.71469 & 1788 & 15.5 & 1830 & 30 & 1753 & 26.5 & 1753.0 & 26.5 & -4.4 & \\
\hline Kiowa_1717_4.FIN2 & 179.5 & 0.37 & 0.1078 & 0.0009 & 4.83 & 0.05 & 0.3228 & 0.0032 & 0.67208 & 1789 & 8.5 & 1802 & 15.5 & 1756 & 15 & 1756.0 & 15.0 & -2.6 & \\
\hline Kiowa_1717_10.FIN2 & 254 & 0.51 & 0.1096 & 0.00075 & 4.597 & 0.047 & 0.3025 & 0.00345 & 0.79663 & 1745 & 9 & 1700 & 17 & 1789 & 12.5 & 1789.0 & 12.5 & 5.0 & \\
\hline Kiowa_1717_9.FIN2 & 118.1 & 0.74 & 0.1098 & 0.00065 & 4.807 & 0.0345 & 0.314 & 0.00185 & 0.60559 & 1783 & 6 & 1759 & 9 & 1790 & 10.5 & 1790.0 & 10.5 & 1.7 & \\
\hline Kiowa_1717_127.FIN2 & 292 & 0.46 & 0.1108 & 0.00095 & 4.891 & 0.0455 & 0.314 & 0.0033 & 0.64993 & 1796 & 8 & 1757 & 16 & 1799 & 16 & 1799.0 & 16.0 & 2.3 & \\
\hline Kiowa_1717_120.FIN2c & 123.8 & 0.59 & 0.1129 & 0.00165 & 4.08 & 0.055 & 0.2559 & 0.0032 & 0.31196 & 1648 & 10.5 & 1468 & 16.5 & 1836 & 27 & 1836.0 & 27.0 & 20.0 & Core \\
\hline Kiowa_1717_6.FIN2 & 68.3 & 1.34 & 0.115 & 0.00125 & 5.4 & 0.06 & 0.3395 & 0.00385 & 0.51997 & 1878 & 9.5 & 1880 & 18.5 & 1870 & 19.5 & 1870.0 & 19.5 & -0.5 & \\
\hline Kiowa_1717_84.FIN2 & 84.1 & 2.36 & 0.1147 & 0.0007 & 5.308 & 0.033 & 0.3314 & 0.00205 & 0.49539 & 1869 & 5 & 1844 & 10 & 1872 & 11.5 & 1872.0 & 11.5 & 1.5 & \\
\hline Kiowa_1717_5.FIN2 & 171.7 & 0.19 & 0.1172 & 0.001 & 4.639 & 0.047 & 0.2856 & 0.00275 & 0.63332 & 1752 & 8.5 & 1618 & 14 & 1906 & 15 & 1906.0 & 15.0 & 15.1 & \\
\hline Kiowa_1717-108.FIN2 & 747 & 0.05 & 0.1192 & $\begin{array}{r}0.0016 \\
0.0125\end{array}$ & 4.94 & 0.075 & 0.293 & 0.00485 & 0.6978 & 1797 & 13 & 1650 & 24 & 1917 & 23 & 1917.0 & 23.0 & 13.9 & \\
\hline Kiowa_1717_26.FIN2 & 146 & 0.06 & 0.1193 & 0.00125 & 4.99 & 0.09 & 0.308 & 0.0055 & 0.83328 & 1804 & 15 & 1724 & 27.5 & 1933 & 18 & 1933.0 & 18.0 & 10.8 & \\
\hline $\begin{array}{l}\text { Kowa_-1717-1111.FIN2 } \\
\text { Kolowa } 1717 \text { 125 FIN2 }\end{array}$ & $\begin{array}{l}99.5 \\
709\end{array}$ & $\begin{array}{l}0.45 \\
0.22\end{array}$ & 0.1212 & 0.00175 & 4.83 & 0.08 & 0.2828 & $\begin{array}{r}0.0045 \\
0.0365\end{array}$ & $\begin{array}{r}0.6017 \\
071558\end{array}$ & 1778 & 14.5 & 1605 & 23 & 1940 & 25.5 & 1940.0 & 25.5 & 17.3 & \\
\hline Kiowa_1717-125.FIN2 & $\begin{array}{r}709 \\
648\end{array}$ & 0.22 & 0.1301 & 0.00095 & 6.29 & 0.06 & 0.3429 & 0.00365 & 0.71558 & 2015 & 8 & 1898 & 17.5 & 2094 & 12.5 & 2094.0 & 12.5 & 9.4 & \\
\hline $\begin{array}{l}\text { Kiowa_1717_16.FIN2 } \\
\text { Kiowa 1717 34.FIN2 }\end{array}$ & $\begin{array}{r}64.8 \\
2316\end{array}$ & $\begin{array}{l}0.75 \\
102\end{array}$ & 0.1783 & 0.001 & $\begin{array}{l}12.42 \\
1328\end{array}$ & 0.075 & 0.4962 & 0.00295 & 0.55439 & 2634 & 5.5 & 2595 & 12.5 & 2634 & 9.5 & 2634.0 & 9.5 & 1.5 & \\
\hline $\begin{array}{l}\text { Kiowa-1717-34.FIN2 } \\
\text { Kiowa 1717 30.FIN2 }\end{array}$ & $\begin{array}{l}231.6 \\
105.2\end{array}$ & $\begin{array}{l}1.02 \\
0.82\end{array}$ & $\begin{array}{r}0.1835 \\
0.202\end{array}$ & $\begin{array}{l}0.0008 \\
0.0024\end{array}$ & $\begin{array}{r}13.28 \\
14.2\end{array}$ & $\begin{array}{l}0.075 \\
0.225\end{array}$ & $\begin{array}{l}0.5322 \\
0.512\end{array}$ & $\begin{array}{l}0.0025 \\
0.0055\end{array}$ & $\begin{array}{l}0.65683 \\
0.56842\end{array}$ & $\begin{array}{l}2697 \\
2747\end{array}$ & $\begin{array}{l}5.5 \\
15\end{array}$ & $\begin{array}{l}2749 \\
2659\end{array}$ & $\begin{aligned} 10.5 \\
24\end{aligned}$ & $\begin{array}{l}2682 \\
2829\end{array}$ & $\begin{array}{r}7 \\
195\end{array}$ & $\begin{array}{r}2682.0 \\
28290\end{array}$ & $\begin{array}{l}7.0 \\
195\end{array}$ & -2.5 & \\
\hline Kiowa_1717_86.FIN2 & 217 & $\begin{array}{l}.02 \\
1.01\end{array}$ & 0.0804 & 0.00095 & $\begin{array}{l}14.2 \\
1.34\end{array}$ & 0.018 & $\begin{array}{r}0.512 \\
0.1199\end{array}$ & $\begin{array}{l}0.0055 \\
0.0016\end{array}$ & $\begin{array}{l}0.56842 \\
0.58667\end{array}$ & $\begin{array}{r}244 \\
860\end{array}$ & $\begin{array}{r}15 \\
8\end{array}$ & $\begin{array}{r}2659 \\
729\end{array}$ & $\begin{array}{r}24 \\
9\end{array}$ & $\begin{array}{l}2829 \\
1191\end{array}$ & $\begin{array}{r}19.5 \\
24\end{array}$ & $\begin{array}{l}2829.0 \\
\text { DISCARD }\end{array}$ & $\begin{array}{l}19.5 \\
\text { \#VALEE! }\end{array}$ & $\begin{array}{l}6.0 \\
152\end{array}$ & \\
\hline Kiowa_1717_92.FIN2 & 400 & 0.51 & 0.0902 & 0.0017 & 0.807 & 0.019 & 0.0649 & 0.0008 & 0.66114 & 593 & 10.5 & 405.1 & 4.95 & 1385 & & DISCARD & \#VALUE! & $\begin{array}{l}75.2 \\
31.7\end{array}$ & \\
\hline e Name: Kiowa-1777 & & & & internal & & internal & & internal & & $207 / 235$ & internal & $206 / 238$ & internal & 207/206 & internal & Best age & internal & & \\
\hline Analysis ID & [U] ppm & \begin{tabular}{|l|l}
$T h / U$ \\
\end{tabular} & \begin{tabular}{l|l|}
$207 / 206$ \\
\end{tabular} & $1 \sigma$ error & $207 / 235$ & 10 error & $206 / 238$ & 10 error & RHO & Age Ma & 10 error & Age (Ma) & $1 \sigma$ error & $\mid$ Age (Ma) & 10 error & (Ma) & $1 \sigma$ error & \% Discordance ${ }^{*}$ & Rim/Core \\
\hline Kiowa_1777_73.FIN2 & 143 & 1.02 & 0.052 & 0.0018 & 0.0732 & 0.00245 & 0.01039 & 0.000105 & 0.043095 & 71.4 & 2.3 & 66.6 & 0.65 & 230 & 65 & 66.6 & 0.7 & 6.7 & \\
\hline Kiowa_1777_81.FIN2 & 324 & 0.71 & 0.0466 & 0.0012 & 0.0704 & 0.00175 & 0.01084 & 0.0001 & 0.023613 & 69 & 1.65 & 69.5 & 0.65 & 45 & 48 & 69.5 & 0.7 & -0.7 & \\
\hline Kiowa_1777_87.FIN2 & 108.4 & 0.98 & 0.0476 & 0.00145 & 0.0725 & 0.0022 & 0.011 & 0.00015 & 0.12339 & 70.8 & 2.1 & 70.5 & 0.95 & 70 & 55 & 70.5 & 1.0 & 0.4 & \\
\hline Kiowa_1777_88.FIN2 & 246 & 0.55 & 0.0478 & 0.00105 & 0.0751 & 0.0016 & 0.01125 & 0.00009 & 0.11423 & 73.3 & 1.5 & 72.1 & 0.6 & 109 & 41.5 & 72.1 & 0.6 & 1.6 & \\
\hline Kiowa_1777_34.FIN2 & 235 & 0.59 & 0.0462 & 0.0011 & 0.0726 & 0.00165 & 0.01144 & 0.00009 & 0.071002 & 70.9 & 1.55 & 73.3 & 0.55 & 23 & 42.5 & 73.3 & 0.6 & -3.4 & \\
\hline Kiowa_1777_135.FIN2 & 590 & 0.53 & 0.0488 & 0.001 & 0.079 & 0.00155 & 0.01157 & 0.000105 & 0.081173 & 77.1 & 1.45 & 74.1 & 0.65 & 126 & 39.5 & 74.1 & 0.7 & 3.9 & \\
\hline Kiowa_1777_115.FIN2 & 270 & 0. & 0.0488 & 0.001 & 0.07 & 0.0015 & 0.01184 & 0.00008 & 0.057553 & 76 & 1.4 & 75.9 & 0.5 & 128 & 39 & 75.9 & 0.5 & 0.1 & \\
\hline Kiowa_1777_71.FIN2 & 1270 & 0. & 0.0515 & 0.00065 & 0.0832 & 0.00105 & 0.01197 & 0.00013 & 0.4858 & 81.1 & 0.95 & 76.7 & 0.8 & 246 & 26 & 76 & 0.8 & 5.4 & \\
\hline Kiowa_1777_8.FIN2 & 89.3 & 0. & 0.0502 & 0.00195 & 0.0838 & 0.0031 & 0.01224 & 0.000155 & 0.000333 & 81.2 & 2.9 & 78 & 1 & 160 & 70 & 78 & 1.0 & 3.4 & \\
\hline Kiowa_1777_129.FIN2 & 2540 & 0.28 & 0.0535 & 0.00135 & 0.0957 & 0.0021 & 0.01292 & 0.00023 & 0.28557 & 92.7 & 1.95 & 82 & 1.45 & 340 & 55 & 82 & 1.5 & 10.8 & \\
\hline Kiowa_1777_67.FIN2 & 1635 & 0. & 0.0517 & 0.0006 & 0.4 & 0.0011 & 0.0134 & 0.000145 & 0.51161 & 91.2 & 1 & 85.8 & 0.95 & 268 & 24.5 & 85.8 & 1.0 & 5.9 & \\
\hline Kiowa_1777_60.FIN2 & 767 & 0.54 & 0.0489 & 0.0006 & 0.0916 & 0.001 & 0.01357 & 0.000095 & 0.10357 & 88 & 0.95 & 86.9 & 0.6 & 138 & 25.5 & 86 & 0.6 & 2.2 & \\
\hline Kiowa_1777_7.FIN2 & 216 & 0.49 & 0.0505 & 0.00115 & 0.0956 & 0.0022 & 0.0137 & 0.00011 & 0.23661 & 92.4 & 2.05 & 87.7 & 0.7 & 191 & 43.5 & 87 & 0.7 & 5.1 & \\
\hline Kiowa_1777_51.FIN2 & 826 & 0.67 & 0.0491 & 0.0005 & 0.0988 & 0.00105 & 0.01432 & 0.00009 & 0.30258 & 95.6 & 0.95 & 91.7 & 0.55 & 151 & 22 & 91.7 & 0.6 & 4.1 & \\
\hline Kiowa_1777_32.FIN2 & 479 & 0.77 & 0.0484 & 0.00065 & 0.0982 & 0.00135 & 0.0145 & 0.00009 & 0.16427 & 95 & 1.25 & 92.8 & 0.6 & 119 & 27 & 92.8 & 0.6 & 2.3 & \\
\hline Kiowa_1777_14.FIN2 & 554 & 0.55 & 0.0498 & 0.0009 & 0.0995 & 0.0018 & 0.01455 & 0.00009 & 0.25011 & 96.1 & 1.6 & 93.1 & 0.6 & 164 & 31 & 93.1 & 0.6 & 3.1 & \\
\hline Kiowa_1777_92.FIN2 & $\begin{array}{l}1510 \\
814\end{array}$ & 0.30 & 0.0501 & 0.0006 & 0.1033 & 0.0016 & 0.0147 & 0.00016 & 0.6139 & 99.7 & 1.45 & 94.1 & 1.05 & 189 & 25.5 & 94.1 & 1.1 & 5.6 & \\
\hline 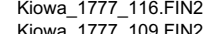 & $\begin{array}{r}814 \\
1730\end{array}$ & 0.48 & $\begin{array}{l}0.04796 \\
0.04913\end{array}$ & $\begin{array}{l}0.000495 \\
0.00465\end{array}$ & $\begin{array}{l}0.0978 \\
0.1013\end{array}$ & $\begin{array}{l}0.001 \\
0.001\end{array}$ & $\begin{array}{l}0.01492 \\
0.01518\end{array}$ & $\begin{array}{l}0.00011 \\
0.00012\end{array}$ & $\begin{array}{l}0.31683 \\
0.4515\end{array}$ & $\begin{array}{c}94.7 \\
98\end{array}$ & $\begin{array}{r}0.9 \\
0.95\end{array}$ & $\begin{array}{c}95.5 \\
97.1\end{array}$ & $\begin{array}{r}0.7 \\
0.75\end{array}$ & $\begin{array}{l}107 \\
151\end{array}$ & $\begin{aligned} 21.5 \\
20\end{aligned}$ & 95.5 & 0.7 & -0.8 & \\
\hline $\begin{array}{l}\text { Kiowa_1777 } 109 \text {.FIN2 } \\
\text { Kiowa 1777 69.FIN2 }\end{array}$ & $\begin{array}{r}1730 \\
243.2\end{array}$ & $\begin{array}{l}0.43 \\
0.56\end{array}$ & $\begin{array}{r}0.04913 \\
0.0507\end{array}$ & $\begin{array}{l}0.000465 \\
0.00095\end{array}$ & 0.1013 & $\begin{array}{l}0.001 \\
0.0021\end{array}$ & $\begin{array}{l}0.01518 \\
0.01564\end{array}$ & $\begin{array}{l}0.00012 \\
0.00016\end{array}$ & $\begin{array}{r}0.4515 \\
0.26912\end{array}$ & $\begin{array}{r}98 \\
1045\end{array}$ & 0.95 & $\begin{array}{r}97.1 \\
100\end{array}$ & 0.75 & $\begin{array}{r}151 \\
218\end{array}$ & $\begin{array}{l}20 \\
38\end{array}$ & 97.1 & 0.8 & $\begin{array}{l}0.9 \\
4.3\end{array}$ & \\
\hline $\begin{array}{l}\text { Klowa_-177_-69.FIN2 } \\
\text { Kiowa_1777_FIN2 }\end{array}$ & $\begin{array}{r}243.2 \\
334\end{array}$ & $\begin{array}{l}0.56 \\
0.73\end{array}$ & $\begin{array}{l}0.040 \% \\
0.0459\end{array}$ & $\begin{array}{l}0.00095 \\
0.0007\end{array}$ & $\begin{array}{l}.1086 \\
0.1019\end{array}$ & $\begin{array}{l}0.0021 \\
0.00165\end{array}$ & $\begin{array}{l}0.01564 \\
0.01608\end{array}$ & $\begin{array}{l}0.00016 \\
0.00012\end{array}$ & $\begin{array}{r}0.26912 \\
0.2314\end{array}$ & $\begin{array}{r}104.5 \\
98.3\end{array}$ & $\begin{array}{r}1.95 \\
1.5\end{array}$ & $\begin{array}{r}100 \\
102.8\end{array}$ & $\begin{array}{r}1 \\
0.75\end{array}$ & $\begin{array}{r}218 \\
16\end{array}$ & $\begin{array}{r}38 \\
29.5\end{array}$ & $\begin{array}{l}100.0 \\
102.8\end{array}$ & $\begin{array}{l}1.0 \\
0.8\end{array}$ & $\begin{array}{l}4.3 \\
-4.6\end{array}$ & \\
\hline Kiowa_1777_29.FIN2 & 686 & 0.47 & 0.049 & 0.0006 & 0.1264 & 0.0015 & 0.0186 & 0.00013 & 0.26994 & 120.7 & 1.35 & 118.8 & 0.85 & 144 & 25.5 & 118.8 & 0.9 & 1.6 & \\
\hline Kiowa_1777_31.FIN2 & 87.7 & 1.48 & 0.0568 & 0.00195 & 0.17 & 0.0055 & 0.02158 & 0.00019 & -0.03007 & 158 & 4.75 & 137.6 & 1.2 & 390 & 65 & 137.6 & 1.2 & 12.9 & \\
\hline Kiowa_1777_82.FIN2 & 343 & 0.89 & 0.0564 & 0.00105 & 0.1696 & 0.00295 & 0.02178 & 0.000305 & 0.22084 & 158.6 & 2.55 & 138.8 & 1.9 & 421 & 39 & 138.8 & 1.9 & 12.5 & \\
\hline Kiowa_1777_65.FIN2 & 128.5 & 3.19 & 0.0537 & 0.00155 & 0.1606 & 0.0046 & 0.02189 & 0.000275 & 0.094078 & 150.8 & 4.05 & 139.6 & 1.75 & 330 & 60 & 139.6 & 1.8 & 7.4 & \\
\hline
\end{tabular}




\begin{tabular}{|c|c|c|c|c|c|c|c|c|c|c|}
\hline Kiowa_1777_83.FIN2 & 317.7 & 2.30 & 0.0479 & 0.0006 & 0.1582 & 0.0019 & 0.02357 & 0.00014 & 0.19833 & 148.9 \\
\hline Kiowa_1777_55.FIN2 & 481.1 & 0.59 & 0.0502 & 0.00065 & 0.1781 & 0.00205 & 0.02527 & 0.00014 & 0.008731 & 166.3 \\
\hline Kiowa_1777_124.FIN2 & 292 & 0.51 & 0.0554 & 0.0007 & 0.2317 & 0.0034 & 0.02985 & 0.000205 & 0.52004 & 211 \\
\hline Kiowa_1777_42.FIN2 & 143.5 & 0.76 & 0.0515 & 0.00085 & 0.2211 & 0.0035 & 0.03109 & 0.000195 & 0.035008 & 202.9 \\
\hline Kiowa_1777_136.FIN2 & 416 & 0.72 & 0.0532 & 0.0007 & 0.294 & 0.00405 & 0.0398 & 0.0006 & 0.51255 & 261 \\
\hline Kiowa_1777_46.FIN2r & 1380 & 0.35 & 0.0521 & 0.0008 & 0.337 & 0.0055 & 0.0464 & 0.0006 & 0.4487 & 294.7 \\
\hline Kiowa_1777_108.FIN2 & 548 & 0.36 & 0.0547 & 0.0008 & 0.35 & 0.005 & 0.0477 & 0.0007 & 0.54088 & 303.5 \\
\hline Kiowa_1777_50.FIN2 & 962 & 0.12 & 0.05336 & 0.000355 & 0.3699 & 0.0029 & 0.04952 & 0.00028 & 0.58877 & 319.4 \\
\hline Kiowa_1777_86.FIN2 & 116.8 & 0.65 & 0.0577 & 0.0009 & 0.405 & 0.0065 & 0.05015 & 0.000345 & 0.33015 & 343.7 \\
\hline Kiowa_1777_93.FIN2 & 1461 & 0.69 & 0.0523 & 0.00055 & 0.3656 & 0.0044 & 0.0503 & 0.00055 & 0.60464 & 315.6 \\
\hline Kiowa_1777_28.FIN2 & 1011 & 0.42 & 0.05687 & 0.000415 & 0.424 & 0.00325 & 0.05378 & 0.0003 & 0.36297 & 358.6 \\
\hline Kiowa_1777_-30.FIN2 & 647 & 0.29 & 0.05382 & 0.000405 & 0.4113 & 0.00315 & 0.05489 & 0.000285 & 0.35365 & 349.4 \\
\hline Kiowa_1777_49.FIN2 & 729 & 1.33 & 0.05417 & 0.00039 & 0.4413 & 0.00315 & 0.05822 & 0.000315 & 0.37656 & 370.8 \\
\hline Kiowa_1777_10.FIN2 & 720 & 0.21 & 0.0631 & 0.0008 & 0.524 & 0.007 & 0.059 & 0.00065 & 0.3343 & 425.9 \\
\hline & 452 & 0.62 & 0.05674 & 0.000365 & 0.4988 & 0.00325 & 0.06461 & 0.000315 & 0.39486 & 410.5 \\
\hline $\begin{array}{l}\text { Kiowa_-1777-1017. FIN2 } \\
\text { Kiowa 177-102.FIN2r }\end{array}$ & $\begin{array}{r}234.6 \\
301\end{array}$ & $\begin{array}{l}2.02 \\
0.12\end{array}$ & $\begin{array}{l}0.0563 \\
0.0628\end{array}$ & $\begin{array}{r}0.0006 \\
0.00315\end{array}$ & $\begin{array}{l}0.513 \\
0.574\end{array}$ & $\begin{array}{l}0.006 \\
0.0245\end{array}$ & $\begin{array}{l}0.0657 \\
0.0671\end{array}$ & $\begin{array}{l}0.00055 \\
0.00305\end{array}$ & $\begin{array}{l}0.46287 \\
0.33131\end{array}$ & $\begin{array}{r}419.2 \\
459\end{array}$ \\
\hline Kiowa_1777_17.FIN2 & 713 & 0.15 & $\begin{array}{l}0.06361 \\
0.0630\end{array}$ & 0.000455 & 0.659 & 0.008 & 0.0755 & 0.0008 & 0.79877 & 512 \\
\hline Kiowa_1777_80.FIN2 & 702 & 0.12 & 0.0634 & 0.0008 & 0.674 & 0.009 & 0.0761 & 0.001 & 0.56561 & 521 \\
\hline Kiowa_1777_117.FIN2 & 66.57 & 2.69 & 0.0614 & 0.00095 & 0.749 & 0.0115 & 0.0889 & 0.0007 & 0.11993 & 565 \\
\hline Kiowa_1777_127.FIN2 & 420 & 0.38 & 0.0598 & 0.0004 & 0.744 & 0.005 & 0.0895 & 0.00055 & 0.49448 & 564.7 \\
\hline Kiowa_1777_27.FIN2 & 86.1 & 1.56 & 0.0618 & 0.0009 & 0.762 & 0.0105 & 0.0897 & 0.0007 & 0.33307 & 573 \\
\hline Kiowa_1777_130.FIN2 & 142 & 0.83 & 0.0715 & 0.001 & 0.908 & 0.012 & 0.0913 & 0.0007 & 0.1946 & 657 \\
\hline Kiowa_1777_39.FIN2 & 144.6 & 0.33 & 0.0606 & 0.0007 & 0.782 & 0.009 & 0.0928 & 0.00075 & 0.44612 & 585 \\
\hline Kiowa_1777_37.FIN2 & 143.8 & 0.53 & 0.06108 & 0.000485 & 0.791 & 0.0075 & 0.0933 & 0.00065 & 0.65497 & 590.5 \\
\hline Kiowa_1777_98.FIN2 & 148.8 & 0.89 & 0.0594 & 0.00065 & 0.771 & 0.0095 & 0.0933 & 0.00085 & 0.55287 & 581 \\
\hline Kiowa_1777_-72.FIN2 & 230 & 1.51 & 0.05849 & 0.000465 & 0.772 & 0.0055 & 0.0958 & 0.00055 & 0.27705 & 580 \\
\hline Kiowa_1777_46.FIN2C & 734 & 0.39 & 0.0601 & 0.0005 & 0.807 & 0.0055 & 0.0959 & 0.0006 & 0.26216 & 600.3 \\
\hline Kiowa_1777_79.FIN2 & 142.8 & 1.20 & 0.0634 & 0.0007 & 0.875 & 0.0095 & 0.0982 & 0.00085 & 0.42872 & 639 \\
\hline Kiowa_1777_77.FIN2 & 131.9 & 0.71 & 0.0637 & 0.00075 & 0.961 & 0.015 & 0.1084 & 0.001 & 0.62 & 681 \\
\hline Kiowa_1777_102.FIN2c & 616.5 & 0.60 & 0.0664 & 0.0006 & 1.01 & 0.012 & 0.1097 & 0.00085 & 0.66683 & 708 \\
\hline Kiowa_1777_110.FIN2 & 279 & 0.51 & 0.0733 & 0.00135 & 1.323 & 0.04 & 0.1339 & 0.0041 & 0.79236 & 842 \\
\hline Kiowa_1777_19.FIN2r & 776 & 0.29 & 0.0756 & 0.0012 & 1.453 & 0.0295 & 0.1402 & 0.00275 & 0.67514 & 910 \\
\hline Kiowa_1777_134.FIN2 & 181.6 & 0.60 & 0.07099 & 0.00049 & 1.458 & 0.015 & 0.147 & 0.00135 & 0.76504 & 912 \\
\hline Kiowa_1777_104.FIN2 & 195.7 & 0.77 & 0.0721 & 0.00055 & 1.667 & 0.012 & 0.1677 & 0.00105 & 0.32403 & 994.3 \\
\hline Kiowa_1777_13.FIN2 & 117.1 & 0.86 & 0.0728 & 0.0006 & 1.477 & 0.013 & 0.1474 & 0.0011 & 0.53305 & 919 \\
\hline Kiowa_1777_139.FIN2 & 599 & 0.41 & 0.0726 & 0.00085 & 1.713 & 0.027 & 0.1703 & 0.00265 & 0.64258 & 1011 \\
\hline Kiowa_1777_33.FIN2 & 131.9 & 0.57 & 0.07388 & 0.00045 & 1.697 & 0.011 & 0.1648 & 0.00075 & 0.39075 & 1006.1 \\
\hline Kiowa_1777_24.FIN2 & 327 & 0.34 & 0.07384 & 0.00039 & 1.681 & 0.0095 & 0.1644 & 0.0008 & 0.66195 & 1000.3 \\
\hline Kiowa_1777_111.FIN2 & 97.7 & 0.51 & 0.0763 & 0.0009 & 1.762 & 0.022 & 0.1718 & 0.0022 & 0.57132 & 1027 \\
\hline Kiowa_1777_-78.FIN2 & 50.5 & 1.09 & $\begin{array}{r}0.0763 \\
0.07679\end{array}$ & 0.0009 & $\begin{array}{r}1.618 \\
178\end{array}$ & 0.0185 & 0.1524 & 0.00085 & 0.17475 & 977 \\
\hline Kiowa_1777_58.FIN2 & 381 & 0.87 & 0.07679 & 0.000455 & $\begin{array}{r}1.78 \\
1787\end{array}$ & 0.0105 & 0.1669 & 0.0008 & 0.52031 & 1037 \\
\hline $\begin{array}{l}\text { Kiowa_ } 1777 \text {-57.FIN2 } \\
\text { Kiowa } 1777 \text { 9.FIN2 }\end{array}$ & $\begin{array}{l}519 \\
216\end{array}$ & $\begin{array}{l}0.44 \\
0.68\end{array}$ & $\begin{array}{l}0.07679 \\
0.07694\end{array}$ & $\begin{array}{r}0.00033 \\
0.000485\end{array}$ & $\begin{array}{l}1.787 \\
1.851\end{array}$ & $\begin{array}{r}0.008 \\
0.0125\end{array}$ & 0.1671 & $\begin{array}{r}0.0008 \\
0.0095\end{array}$ & $\begin{array}{l}0.58881 \\
051526\end{array}$ & 1039.9 \\
\hline $\begin{array}{l}\text { Kowa }-1777-\text { 9.FIIII } \\
\text { Kiowa } 132 \text {.FIN2 }\end{array}$ & $\begin{array}{r}216 \\
102.6\end{array}$ & $\begin{array}{l}0.68 \\
0.44\end{array}$ & $\begin{array}{l}0.00694 \\
0.0778\end{array}$ & $\begin{array}{r}0.000485 \\
0.00065\end{array}$ & 2.851 & $\begin{array}{l}0.0725 \\
0.0215\end{array}$ & $\begin{array}{l}0.1737 \\
0.1906\end{array}$ & $\begin{array}{l}0.000095 \\
0.0017\end{array}$ & $\begin{aligned} 0.51526 \\
0.6467\end{aligned}$ & $\begin{array}{r}1062.2 \\
1139\end{array}$ \\
\hline Kiowa_1777_40.FIN2 & 141.7 & 0.37 & 0.0804 & 0.0006 & 2.074 & 0.017 & 0.1861 & 0.00095 & 0.37423 & 1139 \\
\hline Kiowa 1777 99.FIN2 & 336 & 0.44 & 0.082 & 0.0006 & 2.028 & 0.0165 & 0.1769 & 0.0014 & 0.61606 & 1124 \\
\hline Kiowa_1777_95.FIN2 & 85.5 & 0.65 & 0.0848 & 0.00075 & 2.412 & 0.023 & 0.2044 & 0.0016 & 0.51484 & 1245 \\
\hline Kiowa_1777_133.FIN2 & 462 & 0.67 & 0.0851 & 0.001 & 2.505 & 0.0385 & 0.2134 & 0.00325 & 0.6838 & 1267 \\
\hline Kiowa_1777_59.FIN2 & 51 & 0.27 & 0.089 & 0.00175 & 2.142 & 0.048 & 0.1743 & 0.00115 & 0.68183 & 1149 \\
\hline Kiowa_1777_114.FIN2 & 183.5 & 0.35 & 0.08686 & 0.00049 & 2.537 & 0.022 & 0.2135 & 0.0019 & 0.76761 & 1282 \\
\hline Kiowa_1777_45.FIN2 & 278 & 0.40 & 0.08836 & 0.00048 & 2.778 & 0.016 & 0.2256 & 0.00115 & 0.51772 & 1348.3 \\
\hline Kiowa_1777_48.FIN2 & 203 & 1.38 & 0.089 & 0.0006 & 3.093 & 0.0365 & 0.2483 & 0.0031 & 0.85083 & 1427 \\
\hline Kiowa_1777_1.FIN2 & 112.3 & 0.68 & 0.0893 & 0.00055 & 2.842 & 0.0195 & & 0.00105 & 0.44975 & 1365 \\
\hline Kiowa_1777_97.FIN2 & 251 & 1.12 & 0.0896 & 0.00075 & 3.045 & 0.034 & 43 & 0.0027 & 0.73142 & 1414 \\
\hline Kiowa_1777_119.FIN2 & 122.2 & 1.26 & 0.0903 & 0.00065 & 2.8 & 0.0 & & 0.0014 & 0.5211 & 1354 \\
\hline Kiowa_1777_126.F & 441 & 0.34 & 0.0905 & 0.0009 & & & & & & 1382 \\
\hline Kiowa_1777_91.F & 23 & 0.42 & & 0.000 & & & & & & 1356 \\
\hline Kiowa_1777_1 & 264.7 & 1.53 & 0.0 & 0.00 & & 0.0 & & & & 1363.7 \\
\hline Kiowa_177 & 146.7 & 0.9 & 0.0918 & 0.00 & & & & & 0.45803 & 1342 \\
\hline Kiowa_1777_38.FI & 30 & 0.50 & 0.0919 & 0.000 & 2.59 & 0.0 & & & 0.6918 & 1297.1 \\
\hline Kiowa_17 & 260 & 1.72 & 0.09 & 0.00 & & & & & & 1510 \\
\hline Kiowa_1777_123.FI & 378 & 1.33 & 0.0937 & 0.000 & & 0.0 & & 0.00225 & 0.67874 & 1421 \\
\hline Kiowa_1777_63.FII & 220 & 0.43 & 0.0956 & 0.00055 & 3.40 & 0.015 & & 0.00135 & 0.43898 & 1504.8 \\
\hline Kiowa_1777_2.FIN2 & 295 & 0.88 & 0.09651 & 0.00042 & 3.376 & 0.0 & & 0.0013 & 0.70303 & 1497 \\
\hline Kiowa_1777_19.FIN & 464.9 & 2.37 & 0.0996 & 0.00055 & & 0.035 & & 0.0023 & 0.88118 & 1396 \\
\hline Kiowa_1777_90.FII & 872 & 0.57 & 0.1011 & 0.00075 & 4.125 & 0.0345 & 0.2922 & 0.00275 & & 1656 \\
\hline Kiowa_1777_36.FIN & 317 & 1.29 & 0.10114 & 0.00039 & 3.934 & 0.0165 & & 0.0011 & 0.55953 & 1619.9 \\
\hline $\begin{array}{l}\text { Kiowa_1777_6.FIN2 } 2 \\
\text { Kiowa } 1777 \text { 74 FIN }\end{array}$ & & 0.19 & 0.1017 & 0.00055 & $\begin{array}{r}4.134 \\
297\end{array}$ & 0.046 & 0.2933 & 0.0028 & 0.84328 & 1655 \\
\hline $\begin{array}{l}\text { Kiowa_-17777-74.FIN2 } \\
\text { Kiowa } 1777 \text { 62.FIN2 }\end{array}$ & $\begin{array}{r}124.3 \\
578\end{array}$ & 0.74 & $\begin{array}{r}0.101 \\
0.1018\end{array}$ & $\begin{array}{l}0.000 / 5 \\
0.0007\end{array}$ & $\begin{array}{r}2.97 \\
4.101\end{array}$ & 0.055 & 0.22111 & $\begin{array}{l}0.00036 \\
0.026\end{array}$ & 0.88901 & 1394 \\
\hline $\begin{array}{l}\text { Kowa_-177-62.FIN2 } \\
\text { Kiowa_35.FIN2 }\end{array}$ & $\begin{array}{r}518 \\
268.3\end{array}$ & $\begin{array}{l}0.56 \\
1.57\end{array}$ & $\begin{array}{l}0.1018 \\
0.1021\end{array}$ & $\begin{array}{r}0.00000755 \\
0.004\end{array}$ & $\begin{array}{l}4.101 \\
3.917\end{array}$ & $\begin{array}{l}0.0215 \\
0.0215\end{array}$ & $\begin{array}{l}0.2936 \\
0.2753\end{array}$ & $\begin{array}{l}0.0006 \\
0.0014\end{array}$ & $\begin{array}{l}0.67611 \\
0.7919\end{array}$ & $\begin{array}{l}1652 \\
1615.8\end{array}$ \\
\hline Kiowa_1777_103.FIN2 & 1114 & 0.13 & 0.10226 & 0.000445 & 3.529 & 0.0315 & 0.2504 & 0.00225 & 0.88821 & 1534 \\
\hline Kiowa_1777_120.FIN2 & 327 & 0.60 & 0.1024 & 0.0006 & 3.927 & 0.038 & 0.2785 & 0.00275 & 0.81337 & 1615 \\
\hline Kiowa_1777_16.FIN2 & 173 & 0.70 & 0.1027 & 0.00055 & 4.386 & 0.0355 & 0.3097 & 0.00235 & 0.92303 & 1706 \\
\hline Kiowa_1777_113.FIN2 & 373 & 0.24 & 0.1032 & 0.0006 & 4.039 & 0.028 & 0.2872 & 0.00185 & 0.7192 & 1640 \\
\hline
\end{tabular}

\begin{tabular}{|c|c|c|c|}
\hline 1.65 & 150.2 & 0.9 & 97 \\
\hline 1.75 & 160.9 & 0.9 & 199 \\
\hline 2.8 & 189.6 & 1.3 & 409 \\
\hline & 197.3 & 1.25 & 249 \\
\hline 3.2 & 251.7 & 3.6 & 330 \\
\hline 4.15 & 292.6 & 3.65 & $\begin{array}{l}280 \\
280\end{array}$ \\
\hline 3.8 & 300.2 & 4.35 & $\begin{array}{l}385 \\
227\end{array}$ \\
\hline 2.15 & 311.6 & 1.7 & 337 \\
\hline 4.75 & 315.4 & $\begin{array}{l}2.15 \\
345\end{array}$ & $\begin{array}{r}503 \\
285\end{array}$ \\
\hline 3.25 & 316.1 & 3.45 & 285 \\
\hline 2.3 & 337.7 & 1.85 & 482 \\
\hline 2.25 & 344.4 & 1.75 & 355 \\
\hline 2.2 & 364.7 & 1.95 & 367 \\
\hline 4.7 & 369.3 & 4.1 & 703 \\
\hline 2.2 & 403.5 & 1.9 & 476 \\
\hline 3.85 & 410 & 3.2 & 444 \\
\hline 16 & 418 & 18.5 & 660 \\
\hline 4.75 & 468.8 & 4.95 & 717 \\
\hline 5.5 & 473 & 6 & \\
\hline 6.5 & 548.8 & 4.1 & 622 \\
\hline 3.1 & $\begin{array}{r}552.5 \\
553.3\end{array}$ & 3.25 & $\begin{array}{l}586 \\
624\end{array}$ \\
\hline $\begin{array}{r}6 \\
65\end{array}$ & $\begin{array}{l}553.3 \\
563.1\end{array}$ & $\begin{array}{r}4.2 \\
4.05\end{array}$ & $\begin{array}{l}624 \\
961\end{array}$ \\
\hline 5 & 572 & 4.3 & 604 \\
\hline 4.3 & 574.6 & 3.7 & 628 \\
\hline 6 & 575 & 5 & \\
\hline 3.3 & 589.5 & 3.3 & \\
\hline 3.2 & 590.2 & 3.7 & 602 \\
\hline 5.5 & 604 & 5 & 71 \\
\hline 8 & 663 & 5.5 & 71 \\
\hline 6 & 671 & 5 & 81 \\
\hline 17.5 & 806 & $\begin{array}{r}23 \\
\end{array}$ & \\
\hline 12 & 846 & $\begin{array}{r}15.5 \\
75\end{array}$ & $\begin{array}{r}1079 \\
951\end{array}$ \\
\hline $\begin{array}{r}6 \\
455\end{array}$ & 883 & $\begin{array}{r}7.5 \\
55\end{array}$ & $\begin{array}{l}951 \\
977\end{array}$ \\
\hline 4..00 5 & 886 & $\begin{array}{r}.5 \\
6\end{array}$ & 999 \\
\hline 10.5 & 1012 & 14.5 & 1002 \\
\hline 4.2 & 983.2 & 4.05 & 1031 \\
\hline 3.5 & 980.8 & 4.3 & 1034 \\
\hline 8 & 1021 & 12 & 1077 \\
\hline 7.5 & 914.4 & 4.7 & 1094 \\
\hline 3.95 & 994.7 & 4.35 & 1109 \\
\hline 2.9 & 996.1 & 4.5 & 1112 \\
\hline 4.35 & 1032 & 5 & 1114 \\
\hline $\begin{array}{r}7 \\
55\end{array}$ & $\begin{array}{l}1123 \\
1100\end{array}$ & $\begin{array}{l}9 \\
5\end{array}$ & $\begin{array}{l}1122 \\
1200\end{array}$ \\
\hline 5.5 & 1049 & $\begin{array}{l}5 \\
8\end{array}$ & 1240 \\
\hline 6.5 & 1198 & 8.5 & 1297 \\
\hline 11 & 1243 & 17 & 130 \\
\hline 13 & 1035 & 6.5 & 134 \\
\hline & 1247 & 10 & 135 \\
\hline 4.25 & 1311 & 6 & 1385 \\
\hline 9 & 1426 & 16 & 140 \\
\hline 5 & 1329 & 5.5 & 140 \\
\hline 8.5 & 1407 & 14 & 14 \\
\hline 6 & 1312 & 7.5 & 142 \\
\hline 11.5 & 1343 & 18 & \\
\hline 7.5 & 1295 & 11 & \\
\hline 4.4 & 1306 & 7 & 14 \\
\hline - & 1255 & 7 & \\
\hline 4.5 & 1196 & 7 & \\
\hline 11.5 & 1513 & 20 & \\
\hline 8.5 & 1362 & 12 & $\begin{array}{l}1499 \\
1539\end{array}$ \\
\hline 4.55 & $\begin{array}{l}1487 \\
1447\end{array}$ & $\begin{array}{r}7 \\
6\end{array}$ & \\
\hline 4.85 & 1443 & 6.5 & $\begin{array}{l}1556 \\
\end{array}$ \\
\hline 9 & $\begin{array}{l}1262 \\
162\end{array}$ & 12 & 1616 \\
\hline 7 & $\begin{array}{l}1650 \\
1592\end{array}$ & $\begin{array}{r}144 \\
55\end{array}$ & \\
\hline 3.35 & $\begin{array}{l}1592 \\
1692\end{array}$ & $\begin{array}{l}5.5 \\
14\end{array}$ & 643 \\
\hline $\begin{array}{r}9.5 \\
145\end{array}$ & $\begin{array}{l}1656 \\
1233\end{array}$ & $\begin{array}{l}l_{14} \\
19\end{array}$ & \\
\hline $\begin{array}{r}14.5 \\
7\end{array}$ & 1657 & 13 & $\begin{array}{l}1000 \\
1657\end{array}$ \\
\hline 4.45 & 1567 & 7 & \\
\hline 7 & 1439 & 12 & \\
\hline 8 & 1582 & 14 & \\
\hline 7.5 & 1738 & 12 & \\
\hline 5.5 & 1626 & & \\
\hline
\end{tabular}

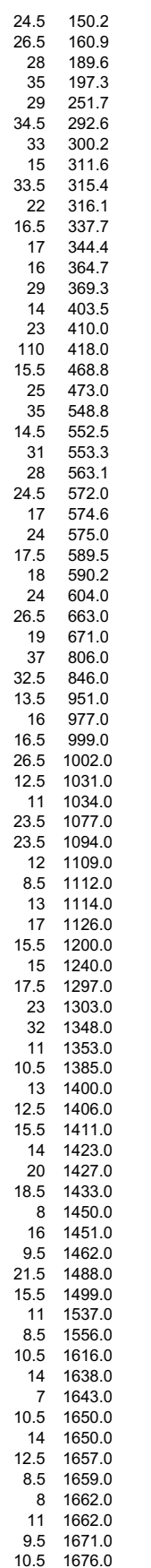




\begin{tabular}{|c|c|c|c|c|c|c|c|c|c|c|c|c|c|c|c|c|c|c|c|}
\hline Kiowa_1777_41.FIN2 & 118.9 & 0.37 & 0.10333 & 0.000475 & 4.51 & 0.027 & 0.315 & 0.00155 & 0.64806 & 1730.9 & 4.85 & 1764 & 7.5 & 1681 & 8.5 & 1681.0 & 8.5 & -4.9 & \\
\hline Kiowa_1777_25.FIN2 & 169 & 0.79 & 0.1038 & 0.00085 & 3.858 & 0.033 & 0.2696 & 0.00195 & 0.55472 & 1601 & 7 & 1538 & 10 & 1685 & 15 & 1685.0 & 15.0 & 8.7 & \\
\hline Kiowa_1777_125.FIN2 & 101.8 & 0.69 & 0.1043 & 0.00085 & 4.046 & 0.042 & 0.2794 & 0.00255 & 0.64456 & 1640 & 8.5 & 1586 & 13 & 1691 & 14.5 & 1691.0 & 14.5 & 6.2 & \\
\hline Kiowa_1777_118.FIN2 & 843 & 0.77 & 0.10401 & 0.00045 & 4.246 & 0.03 & 0.2969 & 0.0021 & 0.74032 & 1681 & 6 & 1675 & 10.5 & 1694 & 8 & 1694.0 & 8.0 & 1.1 & \\
\hline Kiowa_1777_53.FIN2 & 1244 & 0.10 & 0.1045 & 0.0005 & 3.785 & 0.0275 & 0.256 & 0.0019 & 0.75526 & 1588 & 6 & 1468 & 9.5 & 1703 & 9 & 1703.0 & 9.0 & 13.8 & \\
\hline Kiowa_1777_3.FIN2 & 212 & 1.56 & 0.1046 & 0.00055 & 3.929 & 0.032 & 0.2696 & 0.00155 & 0.71716 & 1619 & 7 & 1538 & 8 & 1704 & 9.5 & 1704.0 & 9.5 & 9.7 & \\
\hline Kiowa_1777_121.FIN2 & 221.2 & 0.66 & 0.1055 & 0.0011 & 3.662 & 0.0425 & 0.2537 & 0.0029 & 0.60717 & 1559 & 9 & 1458 & 14.5 & 1708 & 19.5 & 1708.0 & 19.5 & 14.6 & \\
\hline Kiowa_1777_54.FIN2 & 452 & 0.44 & 0.10493 & 0.00046 & 4.193 & 0.0235 & 0.2845 & 0.0016 & 0.69874 & 1671.5 & 4.6 & 1613 & 8 & 1711 & 8 & 1711.0 & 8.0 & 5.7 & \\
\hline Kiowa_-1777-47.FIN2 & 1057 & 0.21 & 0.10489 & 0.00039 & 4.209 & 0.018 & 0.2868 & 0.00155 & 0.69731 & 1676.3 & 3.35 & 1625 & 7.5 & 1713 & 7 & 1713.0 & 7.0 & 5.1 & \\
\hline Kiowa_1777_106.FIN2 & 541 & 0.17 & 0.1061 & 0.001 & 4.08 & 0.055 & 0.2815 & 0.00335 & 0.72903 & 1648 & 11 & 1596 & 17 & 1719 & 17.5 & 1719.0 & 17.5 & 7.2 & \\
\hline Kiowa_1777_131.FIN2 & $\begin{array}{r}274 \\
1045\end{array}$ & 0.71 & 0.1065 & 0.00125 & 4.11 & 0.055 & 0.2801 & 0.0041 & 0.63809 & 1652 & 11.5 & 1592 & 20 & 1729 & 21.5 & 1729.0 & 21.5 & 7.9 & \\
\hline Kiowa_1777_94.FIN2 & $\begin{array}{r}104.5 \\
365\end{array}$ & 0.54 & $\begin{array}{r}0.1072 \\
0.10691\end{array}$ & 0.0012 & $\begin{array}{r}4.32 \\
\end{array}$ & $\begin{array}{r}0.065 \\
0.075\end{array}$ & 0.2895 & 0.004 & 0.71024 & 1690 & 12.5 & 1634 & 20 & 1736 & 20.5 & 1736.0 & 20.5 & 5.9 & \\
\hline $\begin{array}{l}\text { Kiowa_1777_21.FIN2 } \\
\text { Kiowa 1777 4.FIN2 }\end{array}$ & $\begin{array}{r}365 \\
2261\end{array}$ & 0.62 & 0.10684 & 0.000475 & 4.464 & 0.0275 & 0.305 & 0.00225 & 0.94029 & 1723 & 5 & 1710 & 9.5 & 1744 & 8 & 1744.0 & 8.0 & 1.9 & \\
\hline $\begin{array}{l}\text { Kiowa- } 1777 \text { - } 4 \text {.IN2 } \\
\text { Kiowa } 1777 \text {.FIN2 }\end{array}$ & $\begin{array}{r}226.1 \\
559\end{array}$ & 0.71 & 0.1077 & $\begin{array}{l}0.00075 \\
0.0005\end{array}$ & $\begin{array}{r}4.621 \\
45\end{array}$ & $\begin{array}{r}0.033 \\
0.023\end{array}$ & 0.3067 & 0.00175 & 0.47362 & $\begin{array}{r}1753 \\
17208\end{array}$ & 6 & 1724 & 8.5 & $\begin{array}{l}1756 \\
1758\end{array}$ & 12.5 & $\begin{array}{l}1756.0 \\
17580\end{array}$ & $\begin{array}{l}12.5 \\
8.5\end{array}$ & 1.8 & \\
\hline Kiowa 1777 140.FIN2 & 74.8 & $\begin{array}{l}0.06 \\
0.47\end{array}$ & $\begin{array}{l}0.10 / 7 \\
0.1098\end{array}$ & $\begin{array}{l}0.0005 \\
0.0016\end{array}$ & $\begin{array}{l}4.5 \\
4.8\end{array}$ & $\begin{array}{l}0.023 \\
0.09\end{array}$ & $\begin{array}{r}0.3009 \\
0.324\end{array}$ & $\begin{array}{l}0.00175 \\
0.006\end{array}$ & & $\begin{array}{l}1729.8 \\
1778\end{array}$ & $\begin{array}{l}4.25 \\
165\end{array}$ & 1695 & 8.5 & 1758 & $\begin{array}{r}8.5 \\
265\end{array}$ & $\begin{array}{l}1758.0 \\
1761.0\end{array}$ & $\begin{array}{r}8.5 \\
26.5\end{array}$ & 3.6 & \\
\hline Kiowa 1777 52.FIN2 & 254 & 0.56 & 0.1086 & 0.0006 & 4.511 & 0.027 & $\begin{array}{l}0.2856 \\
0.2956\end{array}$ & $\begin{array}{r}0.00185 \\
0.006\end{array}$ & $\begin{array}{l}0.72301 \\
0.6401\end{array}$ & $\begin{array}{l}1778 \\
1732\end{array}$ & $\begin{array}{r}16.5 \\
5\end{array}$ & $\begin{array}{l}1797 \\
1669\end{array}$ & $\begin{aligned} 29.5 \\
9\end{aligned}$ & $\begin{array}{l}1761 \\
1771\end{array}$ & $\begin{array}{l}26.5 \\
10\end{array}$ & $\begin{array}{l}1761.0 \\
1771.0\end{array}$ & 26.5 & $\begin{array}{l}-2.0 \\
58\end{array}$ & \\
\hline Kiowa_1777_96.FIN2 & 615 & 0.45 & 0.1097 & 0.0011 & 4.249 & 0.043 & 0.2786 & 0.0032 & 0.56441 & 1683 & 8.5 & 1585 & $\begin{array}{c}9 \\
16\end{array}$ & $\begin{array}{l}1771 \\
1783\end{array}$ & 17 & 1783.0 & $\begin{array}{l}10.0 \\
17.0\end{array}$ & $\begin{array}{c}5.8 \\
11 .\end{array}$ & \\
\hline Kiowa_1777_107.FIN2 & 509 & 0.41 & 0.1103 & 0.00105 & 4.18 & 0.05 & 0.2788 & 0.0033 & 0.71025 & 1664 & 10 & 1586 & 17 & 1789 & 17 & 1789.0 & 17.0 & 11.3 & \\
\hline Kiowa_1777_44.FIN2 & 745 & 0.38 & 0.1104 & 0.0006 & 4.633 & 0.026 & 0.3032 & 0.0017 & 0.56355 & 1756.2 & 4.9 & 1707 & 8.5 & 1806 & 9.5 & 1806.0 & 9.5 & 5.5 & \\
\hline Kiowa_1777_128.FIN2 & 780 & 1.13 & 0.1109 & 0.00065 & 5.104 & 0.046 & 0.3283 & 0.00335 & 0.65742 & 1832 & 7.5 & 1827 & 16.5 & 1808 & 11 & 1808.0 & 11.0 & -1.1 & \\
\hline Kiowa_1777_137.FIN2 & 197 & 0.27 & 0.1109 & 0.0009 & 4.729 & 0.0445 & 0.3049 & 0.00265 & 0.58081 & 1768 & 7.5 & 1714 & 13 & 1811 & 15.5 & 1811.0 & 15.5 & 5.4 & \\
\hline Kiowa_1777_11.FIN2 & 263 & 0.08 & 0.1129 & 0.0008 & 4.737 & 0.044 & 0.3017 & 0.00225 & 0.66103 & 1769 & 7.5 & 1701 & 11.5 & 1841 & 13 & 1841.0 & 13.0 & 7.6 & \\
\hline Kiowa_1777_138.FIN2 & 430 & 0.05 & 0.1156 & 0.0013 & 4.45 & 0.075 & 0.2775 & 0.0044 & 0.80188 & 1711 & 15 & 1573 & 22.5 & 1869 & 20.5 & 1869.0 & 20.5 & 15.8 & \\
\hline Kiowa_1777_75.FIN2 & 246.2 & 0.59 & 0.1164 & 0.00115 & 4.45 & 0.055 & 0.2778 & 0.0032 & 0.63505 & 1715 & 10 & 1577 & 16.5 & 1888 & 18.5 & 1888.0 & 18.5 & 16.5 & \\
\hline Kiowa_1777_20.FIN2 & 397.7 & 1.15 & 0.1163 & 0.00065 & 5.214 & 0.0365 & 0.3262 & 0.00225 & 0.78273 & 1852 & 6 & 1818 & 11 & 1897 & 10 & 1897.0 & 10.0 & 4.2 & \\
\hline Kiowa_1777_100.FIN2 & 303 & 0.52 & 0.1192 & 0.00135 & 4.94 & 0.075 & 0.2986 & 0.00435 & 0.72008 & 1803 & 12.5 & 1682 & 21.5 & 1933 & 20.5 & 1933.0 & 20.5 & 13.0 & \\
\hline Kiowa_1777_43.FIN2 & 640 & 0.06 & 0.1203 & 0.0006 & 5.15 & 0.0465 & 0.3079 & 0.00215 & 0.82634 & 1840 & 7.5 & 1729 & 10.5 & 1956 & 9 & 1956.0 & 9.0 & 11.6 & \\
\hline Kiowa_1777_56.FIN2 & 459 & 0.66 & 0.1221 & 0.000445 & 4.997 & 0.0485 & 0.2927 & 0.00255 & 0.92049 & 1814 & 8.5 & 1653 & 12.5 & 1985 & 6.5 & 1985.0 & 6.5 & 16.7 & \\
\hline Kiowa_1777_26.FIN2 & 663 & 0.05 & 0.1407 & 0.0007 & 6.88 & 0.065 & 0.3523 & 0.00255 & 0.84769 & 2093 & 8.5 & 1945 & 12 & 2233 & 9 & 2233.0 & 9.0 & 12.9 & \\
\hline Kiowa_1777_66.FIN2 & 471 & 0.49 & 0.1644 & 0.0008 & 9.94 & 0.06 & 0.444 & 0.0025 & 0.59392 & 2428 & 5.5 & 2367 & 11 & 2501 & 8 & 2501.0 & 8.0 & 5.4 & \\
\hline Kiowa_1777_105.FIN2 & 282 & 0.56 & 0.1665 & 0.0013 & 8.4 & 0.145 & 0.367 & 0.0055 & 0.90365 & 2272 & 16.5 & 2009 & 25.5 & 2518 & 13.5 & 2518.0 & 13.5 & 20.2 & \\
\hline Kiowa_1777_18.FIN2 & 319 & 0.85 & 0.1822 & 0.0014 & 11.53 & 0.105 & 0.4599 & 0.00405 & 0.61042 & 2564 & 8.5 & 2438 & 18 & 2670 & 12.5 & 2670.0 & 12.5 & 8.7 & \\
\hline Kiowa_1777_22.FIN2 & 547.7 & 0.09 & 0.1854 & 0.00075 & 12.71 & 0.075 & 0.4963 & 0.00295 & 0.77961 & 2657 & 5.5 & 2597 & 12.5 & 2700 & 6.5 & 2700.0 & 6.5 & 3.8 & \\
\hline Kiowa_1777_122.FIN2 & 106.7 & 0.98 & 0.1879 & 0.001 & 13.05 & 0.07 & 0.5031 & 0.0029 & 0.51623 & 2681.1 & 4.95 & 2625 & 12.5 & 2720 & 9 & 2720.0 & 9.0 & 3.5 & \\
\hline Kiowa_1777_68.FIN2 & 264 & 1.11 & 0.1926 & 0.00115 & 12.5 & 0.08 & 0.4744 & 0.0029 & 0.5916 & 2641 & 6 & 2502 & 12.5 & 2761 & 9.5 & 2761.0 & 9.5 & 9.4 & \\
\hline Kiowa_1777_23.FIN2 & 648 & 0.31 & 0.0986 & 0.0014 & 1.786 & 0.0485 & 0.1292 & 0.003 & 0.75604 & 1023 & 19.5 & 780 & 17.5 & 1568 & $24 \mathrm{E}$ & DISCARD & \#VALUE! & 23.8 & \\
\hline Kiowa_1777_61.FIN2 & 1017 & 0.12 & 0.071 & 0.00205 & 0.551 & 0.0185 & 0.0579 & 0.00165 & 0.64482 & 441 & 12 & 362 & 10 & 890 & 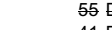 & DISCARD & \#VALUE! & 17.9 & \\
\hline Kiowa_1777_64.FIN2 & 1680 & 0.05 & 0.1616 & 0.0042 & 2.39 & 0.135 & 0.106 & 0.005 & 0.89845 & 1203 & 40.5 & 659 & 33 & 2446 & & DISCARD & \#VALUE! & 45.2 & \\
\hline Kiowa_1777-76.FIN2 & 111 & 1.37 & 0.1166 & 0.0011 & 3.15 & 0.095 & 0.194 & 0.0055 & 0.94488 & 1411 & 24.5 & 1133 & 29.5 & 1894 & & DISCARD & \#УALUE! & 40.2 & \\
\hline Kiowa_1777_112.FIN2 & 780 & 3.51 & 0.1101 & 0.00055 & 3.216 & 0.0415 & 0.2133 & 0.0025 & 0.90972 & 1453 & 10.5 & 1244 & 13.5 & 1796 & 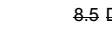 & DISCARD & \#VALUE! & 30.7 & \\
\hline le Name: Kiowa-1864 & & & & internal & & internal & & internal & & $207 / 235$ & internal & $206 / 238$ & internal & 207/206 & internal & Best age & internal & & \\
\hline Analysis ID & [U] ppm & \begin{tabular}{l|l} 
Th/U \\
\end{tabular} & 207/206 & 10 error & 207/235 & $1 \sigma$ error & 206/238 & $1 \sigma$ error & RHO & Age Ma & $1 \sigma$ error & Age (Ma) & $1 \sigma$ error & Age (Ma) & $1 \sigma$ error & (Ma) & $1 \sigma$ error & \% Discordance ${ }^{*}$ & Rim/Core \\
\hline Kiowa-1864-part2_23.FIN2 & & 1.03 & 0.0534 & 0.00365 & 0.08 & 0.006 & 0.01054 & 0.00026 & 0.24025 & 78 & 5.5 & 67.6 & 1.65 & 300 & 135 & 67.6 & 1.7 & 13.3 & \\
\hline Kiowa-1864-part2_26.FIN2 & 86 & 0.70 & 0.0485 & 0.0016 & 0.0763 & 0.0024 & 0.01151 & 0.000125 & 0.073279 & 74.2 & 2.25 & 73.7 & 0.8 & 100 & 60 & 73.7 & 0.8 & 0.7 & \\
\hline Kiowa-1864-part2_17.FIN2 & 345 & 0.66 & 0.0476 & 0.0012 & 0.0784 & 0.0022 & 0.01196 & 0.00021 & 0.52297 & 76.5 & 2.1 & 76.6 & 1.35 & 85 & 49 & 76.6 & 1.4 & -0.1 & \\
\hline Kiowa-1864-part2_3.FIN2 & 734 & 0.65 & 0.0511 & 0.00075 & 0.0851 & 0.0014 & 0.012 & 0.000155 & 0.48647 & 82.8 & 1.35 & 76.9 & 1 & 244 & 32.5 & 76.9 & 1.0 & 7.1 & \\
\hline Kiowa-1864-part1_20.FIN2 & 586 & 0.82 & 0.0499 & 0.00085 & 0.0914 & 0.0017 & 0.01302 & 0.000115 & 0.40851 & 88.7 & 1.55 & 83.4 & 0.7 & 201 & 36 & 83.4 & 0.7 & 6.0 & \\
\hline Kiowa-1864-part1_19.FIN2 & 360 & 1.13 & 0.0483 & 0.00085 & 0.101 & 0.0017 & 0.0149 & 0.00012 & 0.17324 & 97.6 & 1.55 & 95.3 & 0.75 & 126 & 36 & 95.3 & 0.8 & 2.4 & \\
\hline Kiowa-1864-part1_11.FIN2 & 77.9 & 0.75 & 0.0514 & 0.00175 & 0.1174 & 0.0039 & 0.01677 & 0.00026 & 0.23684 & 111.9 & 3.55 & 107.2 & 1.65 & 250 & 65 & 107.2 & 1.7 & 4.2 & \\
\hline Kiowa-1864-part1_14.FIN2 & 184.1 & 1.30 & 0.051 & 0.0009 & 0.1831 & 0.00325 & 0.02605 & 0.000195 & 0.32071 & 171.4 & 2.75 & 165.7 & 1.2 & 219 & 36.5 & 165.7 & 1.2 & 3.3 & \\
\hline Kiowa-1864-part2_39.FIN2 & 819 & 0.65 & 0.05041 & 0.00044 & 0.1814 & 0.0016 & 0.0261 & 0.00016 & 0.49129 & 169.1 & 1.4 & 166.1 & 1 & 204 & 19 & 166.1 & 1.0 & 1.8 & \\
\hline Kiowa-1864-part1_30.FIN2 & 472 & 1.83 & 0.0565 & 0.0008 & 0.2047 & 0.00295 & 0.0262 & 0.000165 & 0.17841 & 188.7 & 2.5 & 166.7 & 1 & 456 & 32 & 166.7 & 1.0 & 11.7 & \\
\hline Kiowa-1864-part2_33.FIN2 & 426 & 0.59 & 0.0501 & 0.00055 & 0.1826 & 0.00205 & 0.02642 & 0.00022 & 0.025792 & 170 & 1.75 & 168.1 & 1.4 & 191 & 23.5 & 168.1 & 1.4 & 1.1 & \\
\hline Kiowa-1864-part2_36.FIN2 & 78 & 0.66 & 0.0512 & 0.00115 & 0.261 & 0.006 & 0.03682 & 0.000315 & 0.19255 & 234.2 & 4.8 & 233.1 & 1.95 & 230 & 45 & 233 & 2.0 & 0.5 & \\
\hline Kiowa-1864-part2_29.FIN2 & 193.3 & 35 & 0.0507 & 0.00065 & 0.26 & 0.00345 & 0.03851 & 0.00027 & -0.10753 & 241.5 & 2.75 & 243.6 & 1.65 & 215 & 26.5 & & 1.7 & -0.9 & \\
\hline Kiowa-1864-part1_22.FIN2 & 118.4 & 1.20 & 0.0521 & 0.00085 & & & 0.04707 & 0.00037 & 0.25254 & 293. & 3.95 & 296.4 & 2.25 & 279 & 34 & & 2.3 & -0.9 & \\
\hline Kiowa-1864-part2_32.FIN2 & 496 & 0.59 & 0.0606 & 0.00085 & 0.445 & 0.0065 & 0.05362 & 0.00039 & 0.22739 & 372. & 4.3 & 336.6 & 2.4 & 596 & 29 & & 2.4 & 9.7 & \\
\hline Kiowa-1864-part2_28.FIN2 & 378 & 0.80 & 0.05454 & 0.00044 & 0.4255 & 0.0037 & 0.05671 & 0.000375 & 0.45845 & 359. & 2.65 & 355.5 & 2.3 & 380 & 18 & & 2.3 & 1.1 & \\
\hline Kiowa-1864-part1_6.FIN2 & 83.9 & 50 & 0.0649 & 0.00085 & 0.874 & 0.0115 & 0.099 & 0.00095 & 0.44827 & 64 & 6.5 & 608 & 5.5 & 770 & 26.5 & 60 & 5.5 & 5.0 & \\
\hline Kiowa-1864-part2_9.FIN2r & 377 & 0.02 & 0.0619 & 0.0007 & 0.871 & 0.01 & 0.1022 & 0.0011 & 0.50686 & 635 & 5.5 & 627 & 6.5 & 661 & 24 & 627.0 & 6.5 & 1.3 & $\operatorname{Rim}$ \\
\hline Kiowa-1864-part2_9.FIN2c & 19.51 & 0.82 & 0.0741 & 0.00155 & 1.759 & 0.037 & 0.1725 & 0.0019 & 0.29253 & 1024 & 13.5 & 1025 & 10.5 & 1006 & 40.5 & 1006.0 & 40.5 & -1.9 & Core \\
\hline Kiowa-1864-part2_18.FIN2 & $\begin{array}{r}244.9 \\
200\end{array}$ & 0.10 & 0.07429 & 0.000465 & 1.841 & 0.0145 & 0.1798 & 0.00105 & 0.57522 & 105 & 5 & 1066 & 5.5 & 1042 & 12.5 & 1042.0 & 12.5 & -2.3 & \\
\hline $\begin{array}{l}\text { Kiowa-1864-part2_1.FIN2 } \\
\text { Kiowa-1864-part_ 33 FFN2 }\end{array}$ & $\begin{array}{l}390 \\
157\end{array}$ & $\begin{array}{l}0.70 \\
0.32\end{array}$ & $\begin{array}{r}0.0766 \\
-0812-1\end{array}$ & 0.00075 & 1.775 & 0.0195 & 0.169 & 0.002 & 0.64323 & 1033 & 7 & 1005 & 11 & 1095 & 19.5 & 1095.0 & 19.5 & 8.2 & \\
\hline & $\begin{array}{r}157 \\
1345\end{array}$ & & $\begin{array}{l}0.0812 \\
0.028\end{array}$ & $\begin{array}{r}0.0005 \\
0.00055\end{array}$ & 2.32 & 0.015 & 0.2076 & 0.0009 & 0.45885 & 1220 & $\begin{array}{r}4.5 \\
4.65\end{array}$ & 1215.6 & 4.85 & 1227 & 12 & 1227.0 & 12.0 & 0.9 & \\
\hline $\begin{array}{l}\text { Kiowa-1864-part2_-57.FIN2 } \\
\text { Kiowa-1864-part1 21.FIN2 }\end{array}$ & $\begin{array}{r}134.5 \\
891\end{array}$ & $\begin{array}{l}0.14 \\
0.48\end{array}$ & $\begin{array}{r}0.0828 \\
0.08742\end{array}-2$ & $\begin{array}{r}0.00055 \\
0.000375\end{array}$ & 2.435 & 0.0155 & 0.2138 & 0.00115 & 0.40941 & $\begin{array}{l}1251.3 \\
14355\end{array}$ & $\begin{array}{l}4.65 \\
3 .\end{array}$ & 1249 & 6 & 1255 & $\begin{array}{r}13 \\
85\end{array}$ & $\begin{array}{l}1255.0 \\
1670\end{array}$ & $\begin{array}{ll}13.0 \\
85\end{array}$ & 0.5 & \\
\hline $\begin{array}{l}\text { Kiowa-1864-part1_21.FIN2 } \\
\text { Kiowa-1864-part2 41.FIN2 }\end{array}$ & $\begin{array}{l}891 \\
140\end{array}$ & $\begin{array}{l}0.48 \\
1.10\end{array}$ & $\begin{array}{l}0.08742 \\
0.08763\end{array}$ & $\begin{array}{l}0.000375 \\
0\end{array}$ & $\begin{array}{l}3.114 \\
2853\end{array}$ & 0.0135 & 0.2581 & 0.00135 & 0.64138 & $\begin{array}{l}1435.5 \\
13685\end{array}$ & $\begin{array}{r}3.3 \\
405\end{array}$ & $\begin{array}{l}1481 \\
1367\end{array}$ & $\begin{array}{r}7 \\
6\end{array}$ & $\begin{array}{l}1367 \\
1371\end{array}$ & $\begin{array}{r}8.5 \\
105\end{array}$ & $\begin{array}{l}1367.0 \\
13710\end{array}$ & $\begin{array}{r}8.5 \\
105\end{array}$ & $\begin{array}{l}-8.3 \\
0.3\end{array}$ & \\
\hline $\begin{array}{l}\text { Kowa-1864-part2_4.1.iN2 } \\
\text { Kiowa-1864-part2_54.FIN2 }\end{array}$ & 73.5 & 1.57 & $\begin{aligned} 0.0863 \\
0.0881\end{aligned}$ & $\begin{array}{l}0.0004 t \\
0.0007\end{array}$ & $\begin{array}{l}2.853 \\
3.043 \\
-100\end{array}$ & $\begin{array}{l}0.0155 \\
0.021\end{array}$ & $\begin{array}{l}0.2363 \\
0.2523\end{array}$ & $\begin{array}{l}0.0013 \\
0.018\end{array}$ & $\begin{array}{l}0.49511 \\
0.34518\end{array}$ & $\begin{array}{r}1368.5 \\
1416\end{array}$ & $\begin{array}{r}4.05 \\
5.5\end{array}$ & $\begin{array}{l}1367 \\
1449\end{array}$ & $\begin{array}{l}6.5 \\
9.5\end{array}$ & $\begin{array}{l}1371 \\
1372\end{array}$ & $\begin{array}{r}10.5 \\
16\end{array}$ & $\begin{array}{l}1371.0 \\
1372.0\end{array}$ & $\begin{array}{l}10.5 \\
16.0\end{array}$ & $\begin{array}{l}0.3 \\
-5.6\end{array}$ & \\
\hline Kiowa-1864-part2_45.FIN2 & 152.4 & 0.53 & 0.0898 & 0.00075 & 3.05 & 0.055 & 0.2467 & 0.00435 & 0.88237 & 1411 & 13 & 1418 & 22 & 1413 & 16 & 1413.0 & 16.0 & -0.4 & \\
\hline Kiowa-1864-part2_12.FIN2 & 490 & 0.23 & 0.08999 & 0.00041 & 2.928 & 0.017 & 0.2358 & 0.00135 & 0.7097 & 1387.7 & 4.4 & 1364 & 7 & 1421 & 9 & 1421.0 & 9.0 & 4.0 & \\
\hline Kiowa-1864-part2_44.FIN2 & 83 & 1.28 & 0.0911 & 0.00055 & 3.295 & 0.0195 & 0.263 & 0.0014 & 0.37407 & 1478.4 & 4.55 & 1505 & 7 & 1441 & 12 & 1441.0 & 12.0 & -4.4 & \\
\hline Kiowa-1864-part2_21.FIN2 & 491 & 0.19 & 0.0915 & 0.00065 & 3.137 & 0.028 & 0.249 & 0.0021 & 0.60717 & 1440 & 7 & 1432 & 11 & 1452 & 13.5 & 1452.0 & 13.5 & 1.4 & \\
\hline Kiowa-1864-part2_48.FIN2 & 170 & 0.34 & 0.0937 & 0.00125 & 2.91 & 0.05 & 0.2262 & 0.0037 & 0.70431 & 1373 & 13.5 & 1310 & 19 & 1482 & 24.5 & 1482.0 & 24.5 & 11.6 & \\
\hline Kiowa-1864-part2_8.FIN2 & 314 & 0.49 & 0.0938 & 0.0006 & 3.465 & 0.03 & 0.2682 & 0.00205 & 0.66582 & 1516 & 6.5 & 1530 & 10.5 & 1500 & 12 & 1500.0 & 12.0 & -2.0 & \\
\hline
\end{tabular}




\begin{tabular}{|c|c|c|c|c|c|c|c|c|c|c|c|c|c|c|c|c|c|c|c|}
\hline Kiowa-1864-part2_31.FIN2 & 423 & 0.45 & 0.0941 & 0.00075 & 3.441 & 0.0325 & 0.2654 & 0.0021 & 0.56429 & 1513 & 7.5 & 1517 & 11 & 1506 & 15 & 1506.0 & 15.0 & -0.7 & \\
\hline Kiowa-1864-part2_37.FIN2 & 442 & 0.17 & 0.1002 & 0.00055 & 3.999 & 0.03 & 0.2898 & 0.0023 & 0.74456 & 1631 & 6 & 1642 & 12 & 1622 & 10.5 & 1622.0 & 10.5 & -1.2 & \\
\hline Kiowa-1864-part1_1.FIN2 & 64.5 & 0.71 & 0.1023 & 0.00065 & 4.083 & 0.0265 & 0.2874 & 0.0016 & 0.44785 & 1650 & 5.5 & 1628 & 8 & 1663 & 12 & 1663.0 & 12.0 & 2.1 & \\
\hline Kiowa-1864-part2_5.FIN2 & 156.2 & 0.28 & 0.103 & 0.00075 & 4.386 & 0.0375 & 0.3103 & 0.00265 & 0.65121 & 1706 & 7 & 1740 & 13 & 1669 & 13.5 & 1669.0 & 13.5 & -4.3 & \\
\hline Kiowa-1864-part2_22.FIN2 & 243 & 0.09 & 0.1027 & 0.0008 & 4.281 & 0.041 & 0.3023 & 0.00265 & 0.63834 & 1687 & 7.5 & 1701 & 13 & 1669 & 15.5 & 1669.0 & 15.5 & -1.9 & \\
\hline Kiowa-1864-part2_27.FIN2 & 345 & 0.54 & 0.10264 & 0.00047 & 3.706 & 0.0185 & 0.2554 & 0.0013 & 0.62234 & 1571.4 & 4 & 1466 & 6.5 & 1669 & 8.5 & 1669.0 & 8.5 & 12.2 & \\
\hline Kiowa-1864-part2_55.FIN2 & 72.1 & 1.26 & 0.1033 & 0.0008 & 4.243 & 0.035 & 0.2988 & 0.00215 & 0.50935 & 1680 & 7 & 1684 & 11 & 1674 & 14 & . 1674.0 & 14.0 & -0.6 & \\
\hline Kiowa-1864-part2_7.FIN2 & 69.8 & 0.84 & 0.1036 & 0.0006 & 4.401 & 0.0255 & 0.3082 & 0.00155 & 0.45284 & 1710.7 & 4.85 & 1731 & 7.5 & 1683 & 11 & 1683.0 & 11.0 & -2.9 & \\
\hline Kiowa-1864-part2-16.FIN2 & 889 & 0.09 & $\begin{array}{r}0.1035 \\
0.10353\end{array}$ & 0.0005 & 4.04 & 0.0335 & 0.2839 & 0.002 & 0.81899 & 1639 & 7 & 1610 & 10 & 1686 & 9 & 1686.0 & 9.0 & 4.5 & \\
\hline Kiowa-1864-part1_ 2.FIN2 & 344 & 0.62 & 0.10353 & 0.000445 & 4.403 & 0.019 & 0.307 & 0.00115 & 0.50243 & 1712.9 & 3.6 & 1726 & 5.5 & 1689 & 7.5 & 1689.0 & 7.5 & -2.2 & \\
\hline Kiowa-1864-part2_-38.FIN2 & 93.2 & 1.32 & 0.1039 & 0.0005 & 4.372 & 0.0235 & 0.3056 & 0.0015 & 0.45999 & 1705.6 & 4.45 & 1720 & 7 & 1691 & 9 & 1691.0 & 9.0 & -1.7 & \\
\hline Kiowa-1864-part2_-43.FIN2 & 391 & 0.30 & 0.1042 & 0.00065 & 4.409 & 0.0305 & 0.3074 & 0.0021 & 0.58537 & 1713 & 6 & 1726 & 10.5 & 1693 & 12 & 1693.0 & 12.0 & -1.9 & \\
\hline Kiowa-1864-part2_-11.FIN2 & 234 & 0.31 & 0.10407 & 0.000455 & 4.516 & 0.027 & 0.313 & 0.0018 & 0.77943 & 1734 & 5 & 1759 & 8 & 1695 & 8 & 1695.0 & 8.0 & -3.8 & \\
\hline $\begin{array}{l}\text { Kiowa-1864-part2_56.FIN2 } \\
\text { Kiowa-1864-part1 } 35 \text { FIN }\end{array}$ & 184.3 & 0.76 & 0.1046 & 0.00065 & 4.197 & 0.028 & 0.2917 & 0.0021 & 0.16464 & 1671 & 5.5 & 1651 & 10.5 & 1701 & 11.5 & 1701.0 & 11.5 & 2.9 & \\
\hline $\begin{array}{l}\text { Kiowa-1864-part1-35.FIN2 } \\
\text { Kiowa-1864-part1 } 4 \text { FN }\end{array}$ & 57.4 & 1.09 & 0.1052 & 0.00095 & 4.161 & 0.038 & 0.2854 & 0.0021 & 0.44652 & 1666 & 7.5 & 1617 & 10.5 & 1709 & 16.5 & 1709.0 & 16.5 & 5.4 & \\
\hline Kiowa-1864-part1_4.FIN2 & 291 & 0.09 & 0.1048 & 0.0008 & 4.482 & 0.0395 & 0.3087 & 0.00295 & 0.63198 & 1727 & 7.5 & 1732 & 14.5 & 1711 & 14.5 & 1711.0 & 14.5 & -1.2 & \\
\hline Kiowa-1864-part2_51.FIN2 & 119.3 & 0.93 & 0.1056 & 0.00055 & 4.292 & 0.0265 & 0.2952 & 0.00175 & 0.68179 & 1691 & 5 & 1666 & 8.5 & 1721 & 9.5 & 1721.0 & 9.5 & 3.2 & \\
\hline Kiowa-1864-part1_3.FIN2 & 301 & 0.20 & 0.1057 & 0.0011 & 4.45 & 0.115 & 0.308 & 0.0085 & 0.88444 & 1715 & 21 & 1725 & 40.5 & 1726 & 20 & 1726.0 & 20.0 & 0.1 & \\
\hline Kiowa-1864-part2_34.FIN2 & 450 & 0.28 & 0.1064 & 0.0005 & 4.791 & 0.022 & 0.3272 & 0.0017 & 0.50902 & 1782.2 & 3.8 & 1824 & 8 & 1734 & 9 & 1734.0 & 9.0 & -5.2 & \\
\hline Kiowa-1864-part2_19.FIN2 & 368 & 0.17 & 0.107 & 0.00085 & 4.132 & 0.0485 & 0.2812 & 0.0037 & 0.78473 & 1654 & 9.5 & 1594 & 18.5 & 1739 & 14.5 & 1739.0 & 14.5 & 8.3 & \\
\hline Kiowa-1864-part2_24.FIN2 & 371 & 0.38 & 0.10673 & 0.000425 & 4.624 & 0.0195 & 0.3139 & 0.00145 & 0.63705 & 1752.6 & 3.55 & 1759 & 7 & 1743 & 7.5 & 1743.0 & 7.5 & -0.9 & \\
\hline Kiowa-1864-part2_20.FIN2 & 120.7 & 0.56 & 0.1082 & 0.00105 & 4.198 & 0.0435 & 0.2828 & 0.00325 & 0.58003 & 1668 & 8.5 & 1602 & 16.5 & 1757 & 18.5 & 1757.0 & 18.5 & 8.8 & \\
\hline Kiowa-1864-part2_25.FIN2 & 167.7 & 0.61 & 0.1077 & 0.00065 & 4.655 & 0.031 & 0.3138 & 0.00175 & 0.48472 & 1757 & 5.5 & 1758 & 8.5 & 1759 & 10.5 & 1759.0 & 10.5 & 0.1 & \\
\hline Kiowa-1864-part1_27.FIN2 & 50.3 & 0.76 & 0.108 & 0.00105 & 4.35 & 0.065 & 0.2909 & 0.00405 & 0.78928 & 1705 & 12.5 & 1651 & 20.5 & 1760 & 17.5 & 1760.0 & 17.5 & 6.2 & \\
\hline Kiowa-1864-part2_46.FIN2 & 404 & 0.49 & 0.1104 & 0.0009 & 4.58 & 0.05 & 0.3015 & 0.0035 & 0.73478 & 1743 & 9 & 1697 & 17.5 & 1801 & 15 & 1801.0 & 15.0 & 5.8 & \\
\hline Kiowa-1864-part2_-10.FIN2 & 333.3 & 1.28 & 0.1119 & 0.0012 & 4.92 & 0.05 & 0.3162 & 0.00285 & 0.49409 & 1804 & 8.5 & 1771 & 14 & 1827 & 19 & 1827.0 & 19.0 & 3.1 & \\
\hline Kiowa-1864-part____FIN2 & 249.4 & 0.19 & 0.12749 & 0.00049 & 6.417 & 0.0295 & 0.3655 & 0.0019 & 0.71222 & 2034.4 & 4.1 & 2007 & 9 & 2063 & 7 & 2063.0 & 7.0 & 2.7 & \\
\hline Kiowa-1864-part1-9.FIN2 & 195 & 0.97 & 0.0633 & 0.00135 & 0.1377 & 0.003 & 0.016 & 0.00017 & 0.074615 & 131.1 & 2.7 & 102.3 & 1.05 & 683 & 45.5 & DISGARD & \#VALUE! & 22.0 & \\
\hline Kiowa-1864-part1_13.FIN2 & 4.85 & 2.79 & 0.393 & 0.007 & 16.76 & 0.37 & 0.308 & 0.005 & 0.49007 & 2896 & 22 & 1730 & 24.5 & 3865 & & DISCARD & \#VALUE! & 55.2 & \\
\hline Kiowa-1864-part1_31.FIN2 & 4.86 & 2.17 & 0.2465 & 0.00415 & 8.42 & 0.125 & 0.2487 & 0.00325 & 0.3222 & 2284 & 14 & 1429 & 16.5 & 3145 & & DISCARD & \#VALUE! & 54.6 & \\
\hline Kiowa-1864-part1_34.FIN2 & 67 & 1.63 & 0.176 & 0.0065 & 0.738 & 0.036 & 0.02998 & 0.000495 & 0.71162 & 546 & 20.5 & 190.4 & 3.1 & 2500 & & DISGARD & \#VALUE! & 65.1 & \\
\hline Kiowa-1864-part2_42.FIN2 & 669 & 0.08 & 0.086 & 0.0007 & 1.491 & 0.021 & 0.1265 & 0.0017 & 0.84639 & 922 & 8.5 & 767 & 9.5 & 1332 & 46.5 & DISCARD & \#VALUE! & 16.8 & \\
\hline le Name: Kiowa-2076 & & & & internal & & internal & & internal & & $207 / 235$ & internal & $206 / 238$ & internal & & nternal & Best age & internal & & \\
\hline Analysis ID & [U] ppm & \begin{tabular}{l|l}
$T h / U$ \\
\end{tabular} & $207 / 206$ & $1 \sigma$ error & 207/235 & 10 error & $206 / 238$ & $1 \sigma$ error & RHO & & & & 10 error & & 10 error & (Ma) & $1 \sigma$ error & \% Discordance & Rim/Core \\
\hline Kiowa-2076 46.FIN2 & 318 & 0.97 & 0.0487 & 0.00115 & 0.0769 & 0.00175 & 0.01131 & 0.000135 & 0.23695 & 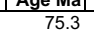 & 1.65 & 72.5 & 0.85 & $\frac{1 \text { Age (ind) }}{5}$ & 46 & $\frac{7 \text { (ina) }}{72.5}$ & 0.9 & 3.7 & Kimilove \\
\hline Kiowa-2076 61.FIN2 & 203 & 0.62 & 0.0522 & 0.00145 & 0.0844 & 0.0023 & 0.01171 & 0.000135 & 0.25634 & 82.4 & 2.15 & 75 & 0.85 & 280 & $\begin{array}{l}40 \\
50\end{array}$ & 75.0 & 0.9 & $\begin{array}{l}.1 . \\
9.0\end{array}$ & \\
\hline Kiowa-2076 80.FIN2 & 680 & 0.44 & 0.0504 & 0.0009 & 0.083 & 0.00135 & 0.01205 & 0.000135 & 0.17297 & $\begin{array}{l}81.4 \\
81.1\end{array}$ & 1.3 & 77.2 & 0.85 & 209 & 36 & 77.2 & $\begin{array}{l}0.9 \\
0.9\end{array}$ & $\begin{array}{l}.0 .0 \\
4.8\end{array}$ & \\
\hline Kiowa-2076 18.FIN2 & 271.7 & 0.74 & 0.0478 & 0.00085 & 0.0851 & 0.00155 & 0.01267 & 0.000095 & 0.18008 & 83.1 & 1.45 & 81.2 & $\begin{array}{l}0.00 \\
0.6\end{array}$ & 117 & 36.5 & 81.2 & 0.6 & 2.3 & \\
\hline Kiowa-2076 11.FIN2 & 188.8 & 0.80 & 0.055 & 0.0014 & 0.1143 & 0.00305 & 0.01504 & 0.00013 & 0.13255 & 108.5 & 2.65 & 96.2 & 0.85 & 344 & 49.5 & 96.2 & 0.9 & 11.3 & \\
\hline Kiowa-2076_50.FIN2 & 95.3 & 0.93 & 0.0502 & 0.00135 & 0.1533 & 0.0039 & 0.02233 & 0.00022 & 0.13361 & 144.7 & 3.5 & 142.4 & 1.4 & 200 & 55 & 142.4 & 1.4 & 1.6 & \\
\hline Kiowa-2076_21.FIN2 & 636 & 0.09 & 0.04882 & 0.00048 & 0.1654 & 0.0016 & 0.02454 & 0.00015 & 0.35937 & 156.1 & 1.45 & 156.3 & 0.95 & 142 & 21 & 156.3 & 1.0 & -0.1 & \\
\hline Kiowa-2076_85.FIN2 & 716 & 0.10 & 0.0506 & 0.000465 & 0.1743 & 0.00165 & 0.02488 & 0.000165 & 0.35943 & 163 & 1.45 & 158.4 & 1.05 & 219 & 20.5 & 158.4 & 1.1 & 2.8 & \\
\hline Kiowa-2076_95.FIN2 & 130.7 & 0.51 & 0.0514 & 0.001 & 0.1779 & 0.0034 & 0.02509 & 0.000175 & 0.036367 & 168 & 2.9 & 159.7 & 1.1 & 259 & 39 & 159.7 & 1.1 & 4.9 & \\
\hline Kiowa-2076_26.FIN2 & 380.9 & 0.77 & 0.0525 & 0.0008 & 0.1848 & 0.0025 & 0.02551 & 0.00026 & 0.2253 & 172.7 & 2.2 & 162.3 & 1.65 & 293 & 32.5 & 162.3 & 1.7 & 6.0 & \\
\hline Kiowa-2076_29.FIN2 & 367 & 0.85 & 0.0504 & 0.0006 & 0.1749 & 0.002 & 0.02554 & 0.000175 & 0.29609 & 163.4 & 1.7 & 162.6 & 1.1 & 203 & 26 & 162.6 & 1.1 & 0.5 & \\
\hline Kiowa-2076_36.FIN2 & 536 & 0.96 & 0.0518 & 0.00065 & 0.1834 & 0.00205 & 0.02569 & 0.000275 & 0.45512 & 171.2 & 1.7 & 163.5 & 1.7 & 276 & 27 & 163.5 & 1.7 & 4.5 & \\
\hline Kiowa-2076_119.FIN2 & 273 & 1.36 & 0.0537 & 0.00095 & 0.1901 & 0.00315 & 0.02596 & 0.000225 & 0.28776 & 176.2 & 2.7 & 165.2 & 1.45 & 331 & 37 & 165.2 & 1.5 & 6.2 & \\
\hline Kiowa-2076_57.FIN2 & 325 & 0.61 & 0.0503 & 0.0008 & 0.1799 & 0.0026 & 0.02618 & 0.000225 & 0.2663 & 167.6 & 2.25 & 166.6 & 1.4 & 194 & 32 & 166.6 & 1.4 & 0.6 & \\
\hline Kiowa-2076_4.FIN2 & 184 & 0.46 & 0.0515 & 0.00225 & 0.197 & 0.008 & 0.027 & 0.0009 & 0.4446 & 182 & 7 & 172 & 5.5 & 260 & 90 & 172.0 & 5.5 & 5.5 & \\
\hline Kiowa-2076_127.FIN2 & 346 & 0.49 & 0.0507 & 0.00065 & 0.1884 & 0.00245 & 0.02752 & 0.0002 & 0.35165 & 175 & 2.05 & 175 & 1.25 & 219 & 27.5 & 175.0 & 1.3 & 0.0 & \\
\hline Kiowa-2076_135.FIN2 & 153 & 0.60 & 0.0513 & 0.0011 & 0.2423 & 0.00465 & 0.03491 & 0.00039 & 0.18893 & 220.2 & 3.7 & 221.2 & 2.4 & 236 & 41.5 & 221.2 & 2.4 & -0.5 & \\
\hline Kiowa-2076_128.FIN2 & 466 & 0.55 & 0.05311 & 0.000485 & 0.3348 & 0.00305 & 0.04595 & 0.000325 & 0.37369 & 293.3 & 2.3 & 289.6 & 2 & 327 & 20.5 & 289.6 & 2.0 & 1.3 & \\
\hline Kiowa-2076_64.FIN2 & 284.9 & 0.59 & 0.05598 & 0.000465 & 0.4992 & 0.00385 & 0.0646 & 0.000295 & 0.32796 & 410.8 & 2.6 & 403.5 & 1.8 & 442 & 18 & 403.5 & 1.8 & 1.8 & \\
\hline Kiowa-2076_75.FIN2 & 160.5 & 0.92 & 0.0571 & 0.00095 & 0.526 & 0.009 & 0.0664 & 0.0008 & 0.4178 & 428 & 6 & 414.6 & 4.75 & 472 & 38 & 414.6 & 4.8 & 3.1 & \\
\hline Kiowa-2076_65.FIN2 & 423.4 & 0.74 & 0.05621 & 0.00046 & 0.5343 & 0.00385 & 0.06901 & 0.00034 & 0.22164 & 434.3 & 2.55 & 430.6 & 2.1 & 455 & 18 & 430.6 & 2.1 & 0.9 & \\
\hline Kiowa-2076_34.FIN2 & 190 & 1.14 & 0.0554 & 0.00065 & 0.538 & 0.006 & 0.07026 & 0.00048 & 0.2354 & 437.1 & 4 & 437.7 & 2.9 & 414 & 26 & 437.7 & 2.9 & -0.1 & \\
\hline Kiowa-2076_32.FIN2 & 110.6 & 0.59 & 0.0571 & 0.0013 & 0.564 & 0.0125 & 0.0727 & 0.0014 & 0.40868 & 450 & 8 & 453 & 8 & 461 & 45 & 453.0 & 8.0 & -0.7 & \\
\hline Kiowa-2076_52.FIN2 & 8.86 & 0.00 & 0.0538 & 0.0025 & 0.562 & 0.028 & 0.0736 & 0.00165 & 0.33808 & 436 & 17.5 & 459 & 9.5 & 280 & 85 & 459.0 & 9.5 & -5.3 & \\
\hline Kiowa-2076_113.FIN2 & 120 & 0.33 & 0.0621 & 0.00095 & 0.68 & 0.009 & 0.0796 & 0.00075 & 0.31551 & 526 & 5.5 & 493.4 & 4.5 & 659 & 31.5 & 493.4 & 4.5 & 6.2 & \\
\hline Kiowa-2076_111.FIN2 & 475 & 1.00 & 0.06288 & 0.00047 & 0.755 & 0.008 & 0.0872 & 0.0008 & 0.72486 & 569.5 & 4.7 & 538.8 & 4.75 & 704 & 16 & 53 & 4.8 & 5.4 & \\
\hline Kiowa-2076_100.FIN2 & 578 & 0.23 & 0.0743 & 0.00075 & 1.075 & 0.014 & 0.1044 & 0.0009 & 0.43829 & 738 & 7 & 640 & 5.5 & 1028 & 19.5 & 640.0 & 5.5 & 13.3 & \\
\hline Kiowa-2076_12.FIN2 & 109.9 & 0.49 & 0.0617 & 0.0008 & 0.907 & 0.0125 & 0.1065 & 0.00105 & 0.48434 & 652 & 6.5 & 652 & 6 & 635 & 28 & 652.0 & 6.0 & 0.0 & \\
\hline Kiowa-2076_62.FIN2 & 135 & 0.41 & 0.0615 & 0.0009 & 0.896 & 0.0125 & 0.1073 & 0.0013 & 0.49496 & 650 & 6.5 & 657 & 7.5 & 645 & 30 & 657.0 & 7.5 & -1.1 & \\
\hline Kiowa-2076_19.FIN2r & 223 & 0.05 & 0.0709 & 0.0036 & 1.19 & 0.06 & 0.13 & 0.0065 & 0.36869 & 790 & 26.5 & 787 & 37.5 & 930 & 110 & 787.0 & 37.5 & 0.4 & Rim \\
\hline Kiowa-2076_110.FIN2 & 96.7 & 0.39 & 0.0718 & 0.0006 & 1.646 & 0.014 & 0.166 & 0.00095 & 0.38752 & 986 & 5.5 & 990 & 5 & 978 & 17 & 978.0 & 17.0 & -1.2 & \\
\hline Kiowa-2076_103.FIN2 & 86.2 & 0.56 & 0.075 & 0.0006 & 1.757 & 0.013 & 0.1726 & 0.00095 & 0.33759 & 1031.2 & 4.85 & 10 & 5.5 & 10 & 16 & 1063.0 & 16 & 3.5 & \\
\hline Kiowa-2076_41.FIN2 & 138.4 & 0.24 & 0.077 & 0.00065 & 1.954 & 0.0145 & 0.1842 & 0.0013 & 0.45418 & 1099.4 & 4.9 & 1090 & 7 & 1119 & 17 & 1119.0 & 17.0 & 2.6 & \\
\hline Kiowa-2076_90.FIN2 & 73.5 & 0.87 & 0.0773 & 0.00075 & 2.09 & 0.021 & 0.196 & 0.00165 & 0.52944 & 1146 & 6.5 & 1153 & 9 & 1126 & 18.5 & 1126.0 & 18.5 & -2.4 & \\
\hline Kiowa-2076_99.FIN2 & 382 & 0.27 & 0.07811 & 0.00032 & 2.161 & 0.0095 & 0.1998 & 0.00085 & 0.54878 & 1167.9 & 3.1 & 1173.8 & 4.6 & 1152 & 8.5 & 1152.0 & 8.5 & -1.9 & \\
\hline Kiowa-2076_82.FIN2 & 258.3 & 0.48 & 0.0784 & 0.00055 & 1.967 & 0.0125 & 0.1826 & 0.0011 & 0.46482 & 1104.2 & 4.25 & 1081 & 6 & 1154 & 13.5 & 1154.0 & 13.5 & 6.3 & \\
\hline Kiowa-2076_133.FIN2 & 230 & 0.80 & 0.0805 & 0.00055 & 2.162 & 0.0135 & 0.1967 & 0.00115 & 0.41857 & 1169.7 & 4.45 & 1158 & 6 & 1204 & 13.5 & 1204.0 & 13.5 & 3.8 & \\
\hline Kiowa-2076_91.FIN2 & 128.5 & 0.12 & 0.081 & 0.00075 & 2.293 & 0.024 & 0.2046 & 0.00185 & 0.65332 & 1207 & 7.5 & 1201 & 10 & 1207 & 18 & 1207.0 & 18.0 & 0.5 & \\
\hline Kiowa-2076_102.FIN2 & 91.2 & 1.00 & 0.0807 & 0.0008 & 2.27 & 0.0225 & 0.2005 & 0.0018 & 0.43057 & 1201 & 7 & 1177 & 9.5 & 1207 & 19 & 1207.0 & 19.0 & 2.5 & \\
\hline Kiowa-2076_67.FIN2 & 238.3 & 1.15 & 0.0806 & 0.00055 & 2.233 & 0.0175 & 0.2003 & 0.0016 & 0.59133 & 1192 & 5.5 & 1176 & 8.5 & 1214 & 14 & 1214.0 & 14.0 & 3.1 & \\
\hline Kiowa-2076_126.FIN2 & 251.3 & 0.91 & 0.08069 & 0.00049 & 2.239 & 0.015 & 0.2019 & 0.0012 & 0.51393 & 1193.7 & 4.75 & 1186 & 6.5 & 1215 & 12 & 1215.0 & 12.0 & 2.4 & \\
\hline
\end{tabular}




\begin{tabular}{|c|c|c|c|c|c|c|c|c|c|c|c|c|c|c|}
\hline Kiowa-2076_71.FIN2 & 493 & 0.24 & 0.0817 & 0.0009 & 1.654 & 0.021 & 0.1483 & 0.0018 & 0.68331 & 989 & 8 & 891 & 10 & 1233 \\
\hline Kiowa-2076_45.FIN2 & 277 & 1.10 & 0.08194 & 0.0004 & 2.21 & 0.017 & 0.1967 & 0.00155 & 0.72738 & 1182 & 5.5 & 1158 & 8.5 & 1245 \\
\hline Kiowa-2076_122.FIN2 & 150 & 1.08 & 0.0822 & 0.00065 & 2.315 & 0.021 & 0.2026 & 0.00135 & 0.54849 & 1216 & 6.5 & 1189 & 7.5 & 1252 \\
\hline Kiowa-2076_105.FIN2 & 124.8 & 0.53 & 0.0823 & 0.00055 & 2.485 & 0.018 & 0.2172 & 0.0013 & 0.48528 & 1267 & 5.5 & 1268 & 7 & 1254 \\
\hline Kiowa-2076_109.FIN2 & 129.7 & 0.65 & 0.0831 & 0.00065 & 2.46 & 0.019 & 0.2134 & 0.00165 & 0.49261 & 1260 & 5.5 & 1246 & 8.5 & 1272 \\
\hline Kiowa-2076_5.FIN2 & 237 & 0.23 & 0.0846 & 0.0009 & 2.23 & 0.055 & 0.196 & 0.00335 & 0.5551 & 1191 & 17.5 & 1151 & 18 & 1292 \\
\hline Kiowa-2076_51.FIN2 & 133.8 & 0.70 & 0.0852 & 0.0013 & 1.781 & 0.0325 & 0.1569 & 0.00185 & 0.46672 & 1036 & 11 & 938 & 10.5 & 1300 \\
\hline Kiowa-2076_116.FIN2 & 124 & 0.46 & 0.0865 & 0.0006 & 2.784 & 0.0225 & 0.2332 & 0.00145 & 0.67297 & 1349 & 6 & 1351 & 7.5 & 1349 \\
\hline Kiowa-2076_60.FIN2 & 332 & 0.77 & 0.08866 & 0.00045 & 2.922 & 0.0175 & 0.2396 & 0.00135 & 0.62869 & 1388.3 & 4.6 & 1384 & 7 & 1395 \\
\hline Kiowa-2076_3.FIN2 & 141 & 0.74 & 0.0901 & 0.0007 & 3.02 & 0.0225 & 0.2426 & 0.00165 & 0.60384 & 1411 & 5.5 & 1400 & 8.5 & 1433 \\
\hline Kiowa-2076_78.FIN2 & 319 & 0.72 & 0.0916 & 0.0009 & 2.917 & 0.0355 & 0.2308 & 0.00295 & 0.65701 & 1387 & 9 & 1347 & 14.5 & 1456 \\
\hline Kiowa-2076_120.FIN2 & 152.9 & 0.94 & 0.0919 & 0.00055 & 2.982 & 0.02 & 0.2358 & 0.0012 & 0.022247 & 1401 & 5 & 1365 & 6 & 1461 \\
\hline Kiowa-2076_123.FIN2 & 276.4 & 0.44 & 0.0919 & 0.00046 & 3.122 & 0.0175 & 0.2477 & 0.0015 & 0.60924 & 1439.5 & 4.15 & 1426 & 8 & 1468 \\
\hline Kiowa-2076_33.FIN2 & 813 & 0.22 & 0.0926 & 0.00065 & 2.919 & 0.022 & 0.2282 & 0.00195 & 0.58845 & 1385 & 5.5 & 1326 & 10.5 & 1474 \\
\hline Kiowa-2076_93.FIN2 & 514 & 0.12 & 0.0974 & 0.0007 & 3.581 & 0.0305 & 0.2676 & $\begin{array}{r}0.0019 \\
0.025\end{array}$ & 0.52564 & 1544 & 6.5 & 1528 & 9.5 & 1570 \\
\hline Kiowa-2076_132.FIN2 & 777 & 0.18 & 0.0975 & 0.00065 & 3.318 & 0.0285 & 0.248 & 0.00255 & 0.66607 & 1485 & 6.5 & 1426 & 13 & 1573 \\
\hline $\begin{array}{l}\text { Kiowa-2076_88.FIN2 } \\
\text { Kiowa-2076 6 FFN2 }\end{array}$ & 84.6 & 0.65 & $\begin{array}{l}0.0975 \\
\end{array}$ & 0.00085 & $\begin{array}{l}3.473 \\
3.151\end{array}$ & 0.038 & 0.2605 & 0.00265 & 0.60497 & 1518 & 8.5 & 1496 & 13 & 1577 \\
\hline $\begin{array}{l}\text { Kiowa-2076_6.FIN2 } \\
\text { Kiowa-2076 } 1 \text { 125.FIN2 }\end{array}$ & 696 & 0.28 & $\begin{array}{r}0.099 \\
0.0992\end{array}$ & 0.00075 & 3.151 & 0.0355 & 0.2312 & 0.00275 & $\begin{array}{l}0.80111 \\
065241\end{array}$ & $\begin{array}{l}1444 \\
155\end{array}$ & 8.5 & 1339 & 14.5 & 1603 \\
\hline $\begin{array}{l}\text { Kiowa-20076-125.FIN2 } \\
\text { Kiowa-2076 24.FIN2 }\end{array}$ & $\begin{array}{l}217 \\
575\end{array}$ & 0.24 & $\begin{array}{l}0.0992 \\
0.0994\end{array}$ & $\begin{array}{l}0.0007 \\
0.0007\end{array}$ & $\begin{array}{l}3.771 \\
3.885\end{array}$ & $\begin{array}{r}0.03 \\
0.035\end{array}$ & & $\begin{array}{l}0.00225 \\
0.0024\end{array}$ & $\begin{array}{l}0.65241 \\
0.66646\end{array}$ & $\begin{array}{l}1585 \\
169\end{array}$ & $\begin{array}{l}6.5 \\
75\end{array}$ & 1576 & $\begin{array}{l}11 \\
12\end{array}$ & $\begin{array}{l}1610 \\
1613\end{array}$ \\
\hline Kiowa-2076 81.FIN2 & $\begin{array}{l}515 \\
681\end{array}$ & $\begin{array}{l}0.25 \\
0.45\end{array}$ & $\begin{array}{l}0.0994 \\
0.1003\end{array}$ & $\begin{array}{l}0.0007 \\
0.0005\end{array}$ & $\begin{array}{l}3.885 \\
3.296\end{array}$ & $\begin{array}{l}0.0345 \\
0.0375\end{array}$ & $\begin{array}{l}0.2851 \\
0.2383\end{array}$ & $\begin{array}{l}0.0024 \\
0.0026\end{array}$ & $\begin{array}{l}0.66646 \\
0.88949\end{array}$ & $\begin{array}{l}1609 \\
1479\end{array}$ & $\begin{array}{r}7.5 \\
9\end{array}$ & 1616 & 12 & $\begin{array}{l}1613 \\
1626\end{array}$ \\
\hline Kiowa-2076_48.FIN2 & 1044 & 0.09 & 0.10133 & 0.00043 & 4.694 & 0.0225 & $\begin{array}{l}0.23367 \\
0.336\end{array}$ & $\begin{array}{l}0.0026 \\
0.0018\end{array}$ & $\begin{array}{l}0.08949 \\
0.66157\end{array}$ & 1765.2 & $\begin{array}{r}9 \\
3.95\end{array}$ & $\begin{array}{l}1870 \\
1870\end{array}$ & $\begin{array}{l}13.5 \\
8.5\end{array}$ & $\begin{array}{l}1626 \\
1646\end{array}$ \\
\hline Kiowa-2076_79.FIN2 & 387 & 1.47 & 0.1017 & 0.0006 & 3.04 & 0.05 & 0.2138 & 0.00305 & 0.91318 & 1408 & 12.5 & 1246 & 16 & 1650 \\
\hline Kiowa-2076_23.FIN2 & 307 & 0.37 & 0.1022 & 0.0006 & 3.842 & 0.0285 & 0.2732 & 0.00225 & 0.71199 & 1602 & 6 & 1558 & 11.5 & 1661 \\
\hline Kiowa-2076_73.FIN2 & 494 & 0.78 & 0.1022 & 0.0007 & 3.971 & 0.029 & 0.282 & 0.00225 & 0.84071 & 1631 & 6.5 & 1600 & 11.5 & 1665 \\
\hline Kiowa-2076_42.FIN2 & 244 & 0.17 & 0.1024 & 0.00085 & 3.824 & 0.0395 & 0.2659 & 0.0029 & 0.80104 & 1593 & 8.5 & 1521 & 15 & 1666 \\
\hline Kiowa-2076_117.FIN2 & 64.8 & 0.86 & 0.1025 & 0.00065 & 4.164 & 0.026 & 0.2942 & 0.00145 & 0.40546 & 1671 & 5 & 1662 & 7.5 & 1667 \\
\hline Kiowa-2076_101.FIN2 & 164.5 & 0.68 & 0.1031 & 0.00095 & 3.478 & 0.047 & 0.2421 & 0.00285 & 0.53043 & 1512 & 9.5 & 1395 & 14.5 & 1668 \\
\hline Kiowa-2076_104.FIN2 & 204 & 0.61 & 0.1023 & 0.00055 & 4.046 & 0.0225 & 0.2871 & 0.0014 & 0.50277 & 1644 & 4.55 & 1626 & 7 & 1670 \\
\hline Kiowa-2076_43.FIN2 & 544 & 0.29 & 0.1027 & 0.00055 & 4.198 & 0.025 & 0.2953 & 0.002 & 0.72723 & 1672.1 & 4.85 & 1667 & 10 & 1671 \\
\hline Kiowa-2076_108.FIN2 & 234 & 0.85 & 0.10272 & 0.00048 & 4.299 & 0.0305 & 0.3024 & 0.0021 & 0.74904 & 1693 & 6 & 1704 & 10.5 & 1674 \\
\hline Kiowa-2076_94.FIN2 & 189.1 & 0.58 & 0.1029 & 0.00055 & 4.247 & 0.0225 & 0.3 & 0.00145 & 0.5017 & 1682.8 & 4.35 & 1692 & 7.5 & 1676 \\
\hline Kiowa-2076_129.FIN2 & 448 & 0.37 & 0.10306 & 0.000495 & 4.363 & 0.029 & 0.305 & 0.00205 & 0.71271 & 1703 & 5.5 & 1717 & 10.5 & 1677 \\
\hline Kiowa-2076_121.FIN2 & 240.3 & 0.61 & 0.1031 & 0.0006 & 4.235 & 0.021 & 0.2981 & 0.00145 & 0.40681 & 1680.7 & 4.15 & 1682 & 7 & 1678 \\
\hline Kiowa-2076_58.FIN2 & 77.2 & 0.87 & 0.1034 & 0.00075 & 4.155 & 0.0335 & 0.2929 & 0.0017 & 0.45013 & 1666 & 6.5 & 1655 & 8.5 & 1681 \\
\hline Kiowa-2076_16.FIN2 & 399.7 & 0.42 & 0.1032 & 0.0007 & 3.482 & 0.03 & 0.2472 & 0.00245 & 0.70554 & 1523 & 6.5 & 1422 & 12.5 & 1684 \\
\hline Kiowa-2076_98.FIN2 & 480 & 1.38 & 0.1034 & 0.00065 & 3.94 & 0.029 & 0.2741 & 0.00175 & 0.50153 & 1620 & 6 & 1561 & 9 & 1684 \\
\hline Kiowa-2076_134.FIN2 & 54.4 & 0.97 & 0.1038 & 0.00095 & 4.163 & 0.0345 & 0.2883 & 0.0019 & 0.30331 & 1664 & 7 & 1634 & 9.5 & 1684 \\
\hline Kiowa-2076_92.FIN2 & 179 & 0.77 & 0.1036 & 0.00065 & 4.289 & 0.0365 & 0.297 & 0.0024 & 0.75109 & 1688 & 7 & 1675 & 12 & 1688 \\
\hline Kiowa-2076_97.FIN2 & 202 & 0.41 & 0.1035 & 0.00085 & 3.657 & 0.0385 & 0.2563 & 0.0029 & 0.74876 & 1564 & 8.5 & 1472 & 15 & 1689 \\
\hline $\begin{array}{l}\text { Koowa-2076_107.FIN2 } \\
\text { Kiowa-206_ 14_FIN2 }\end{array}$ & $\begin{array}{r}191.1 \\
253\end{array}$ & 0.42 & $\begin{array}{l}0.1039 \\
0.1036\end{array}$ & 0.0005 & $\begin{array}{l}4.328 \\
4.23\end{array}$ & 0.021 & $\begin{array}{r}0.302 \\
02978\end{array}$ & 0.00155 & 0.49148 & 1699.4 & 3.85 & 1700 & 7.5 & 1690 \\
\hline $\begin{array}{l}\text { Koowa-2076_114.FIN2 } \\
\text { Kiowa-2076 10 FIN }\end{array}$ & $\begin{array}{r}253 \\
2456\end{array}$ & $\begin{array}{l}0.50 \\
0.18\end{array}$ & $\begin{array}{l}0.1036 \\
0.1041\end{array}$ & $\begin{array}{l}0.0006 \\
0.006\end{array}$ & $\begin{array}{l}4.213 \\
4.253\end{array}$ & $\begin{array}{r}0.024 \\
0.0235\end{array}$ & $\begin{array}{l}0.2978 \\
0.2999\end{array}$ & $\begin{array}{l}0.00195 \\
0.0016\end{array}$ & $\begin{array}{r}0.5886 \\
0.48395\end{array}$ & $\begin{array}{r}1676.2 \\
1683\end{array}$ & $\begin{array}{r}4.55 \\
46\end{array}$ & 1680 & 9.5 & 1694 \\
\hline $\begin{array}{l}\text { Nowa-2016-210.1.FIN2 } \\
\text { Kiowa-2076_55.FIN2 }\end{array}$ & $\begin{array}{r}245.6 \\
489\end{array}$ & 0.53 & $\begin{array}{l}0.1041 \\
0.1041\end{array}$ & $\begin{array}{l}0.0006 \\
0.0009\end{array}$ & $\begin{array}{r}4.253 \\
3.98\end{array}$ & $\begin{array}{r}0.0235 \\
0.04\end{array}$ & $\begin{array}{l}0.2999 \\
0.2767\end{array}$ & $\begin{array}{r}0.0016 \\
0.00275\end{array}$ & $\begin{array}{l}0.48395 \\
0.54502\end{array}$ & $\begin{array}{l}1683 \\
1628\end{array}$ & $\begin{array}{r}4.6 \\
8\end{array}$ & $\begin{array}{l}1670 \\
1576\end{array}$ & $\begin{array}{r}8 \\
135\end{array}$ & $\begin{array}{l}1696 \\
1697\end{array}$ \\
\hline Kiowa-2076_68.FIN2 & 236 & 0.71 & 0.104 & 0.00065 & 4.21 & 0.0305 & 0.2949 & 0.002 & 0.62831 & 1676 & 6 & 1670 & 10.5 & 1702 \\
\hline Kiowa-2076_35.FIN2 & 39.3 & 1.05 & 0.1044 & 0.00095 & 4.184 & 0.037 & 0.2902 & 0.00185 & 0.30312 & 1672 & 7 & 1642 & 9.5 & 1708 \\
\hline Kiowa-2076_44.FIN2 & 940 & 0.06 & 0.1052 & 0.00085 & 4.427 & 0.03 & 0.3084 & 0.00275 & 0.5414 & 1719 & 5.5 & 1731 & 13.5 & 1711 \\
\hline Kiowa-2076_53.FIN2 & 364.7 & 0.57 & 0.1047 & 0.000495 & 4.781 & 0.0305 & 0.3316 & 0.00235 & 0.72826 & 1780 & 5.5 & 1845 & 11.5 & 1712 \\
\hline Kiowa-2076_38.FIN2 & 175.4 & 0.73 & 0.1048 & 0.0006 & 4.275 & 0.0235 & 0.296 & 0.00125 & 0.37951 & 1690.1 & 4.7 & 1671 & 6.5 & 1713 \\
\hline Kiowa-2076_22.FIN2 & 85.7 & 0.80 & 0.1054 & 0.0007 & 4.35 & 0.038 & 0.3025 & 0.00235 & 0.71829 & 1701 & 7 & 1705 & 12 & 1714 \\
\hline Kiowa-2076_87.FIN2 & 113 & 0.66 & 0.1041 & 0.00085 & 4.134 & 0.029 & 0.2905 & 0.0019 & 0.44944 & 1659 & 6 & 1643 & 9.5 & 1714 \\
\hline Kiowa-2076_86.FIN2 & 413 & 0.22 & 0.1053 & 0.00055 & 4.597 & 0.0335 & 0.316 & 0.00195 & 0.77487 & 1746 & 6 & 1772 & 9 & 1715 \\
\hline Kiowa-2076_20.FIN2 & 425.3 & 0.26 & 0.1053 & 0.0007 & 4.46 & 0.0315 & 0.3077 & 0.0024 & 0.67352 & 1723 & 6 & 1733 & 12 & 1718 \\
\hline Kiowa-2076_39.FIN2 & 108.3 & 1.12 & 0.1055 & 0.0007 & 4.421 & 0.035 & 0.3044 & 0.0022 & 0.57011 & 1715 & 6.5 & 1712 & 11 & 1722 \\
\hline Kiowa-2076_17.FIN2 & 148 & 0.97 & 0.1057 & 0.0006 & 4.46 & 0.0255 & 0.3068 & 0.0016 & 0.55285 & 1723.1 & 4.7 & 1724 & 8 & 1724 \\
\hline Kiowa-2076_96.FIN2 & 741 & 0.09 & 0.1062 & 0.00095 & 4.716 & 0.0335 & 0.3292 & 0.00305 & 0.65893 & 1770 & 6 & 183 & 15 & 1725 \\
\hline Kiowa-2076_89.FIN2 & 59.8 & 0.49 & 0.1059 & 0.00075 & 4.615 & 0.0 & 0.31 & & & 17 & 6 & 177 & 11 & 1726 \\
\hline Kiowa-2076_106.FI & 225 & 0.78 & 0.106 & 0.00 & 4.633 & & & 0.00 & 0.52 & 1756 & 4.45 & & 9.5 & 1728 \\
\hline Kiowa-2076_54.FI & 301 & 0.95 & 0.1071 & 0.00 & 4.031 & 0.0335 & 0.2747 & 0.00245 & 0.62571 & 16 & 6.5 & 156 & 12 & 1745 \\
\hline Kiowa-2076_124.FI & 274 & 0.10 & 0.1074 & 0.000 & 4.254 & 0.0405 & 0.2851 & 0.0023 & 0.52881 & 16 & 7.5 & & 11.5 & 1753 \\
\hline Kiowa-2076_15.FIN2 & 210 & 0.48 & 0.1077 & 0.0 & 4.603 & 0.03 & 0.3111 & 0.0021 & 0.5263 & 17 & 6 & & 10.5 & 1761 \\
\hline Kiowa-2076_59.FIN2 & 261 & 0.46 & 0.1074 & 0.000 & 4.328 & 0.03 & 0.294 & 0.00215 & 0.72719 & 16 & 7.5 & & 10.5 & 61 \\
\hline Kiowa-2076_28.FIN2 & 136.4 & 0.57 & 0.1083 & 0.000 & 4.302 & 0.0 & 0.291 & 0.0019 & 0.4124 & 16 & 6.5 & & 9.5 & 1768 \\
\hline Kiowa-2076_1.FIN2 & 102.7 & 0.10 & 0.1085 & 0.001 & 4.48 & 0.065 & 0.296 & 0.004 & 0.67138 & 1726 & 11.5 & 166 & 20 & 1774 \\
\hline Kiowa-2076_70.FIN2 & 314 & 0.90 & 0.1087 & 0.0008 & 4.552 & 0.0355 & 0.2995 & 0.0027 & 0.621 & 1738 & 6.5 & 168 & 13.5 & 77 \\
\hline Kiowa-2076_25.FIN2 & 210 & 1.01 & 0.1087 & 0.00055 & 4.501 & 0.023 & 0.3021 & 0.0016 & 0.50922 & 1731.9 & 4.25 & 1703 & 8 & 81 \\
\hline Kiowa-2076_9.FIN2 & 67.2 & 0.51 & 0.1092 & 0.00115 & 4.459 & $\begin{array}{r}0.046 \\
0.035\end{array}$ & 0.2951 & 0.00295 & 0.63189 & 1719 & 8.5 & 1668 & 14.5 & 1786 \\
\hline $\begin{array}{l}\text { Kiowa-2076_13.FIN2 } \\
\text { KKowa-2076 131 FIN2 }\end{array}$ & 166.4 & 0.81 & $\begin{array}{r}0.11 \\
0.1115\end{array}$ & 0.00075 & $\begin{array}{l}3.691 \\
4585\end{array}$ & 0.0335 & $\begin{array}{r}0.246 \\
0.306\end{array}$ & 0.00235 & $\begin{array}{l}0.73497 \\
054312\end{array}$ & $\begin{array}{l}1566 \\
179\end{array}$ & 7 & 1419 & 12 & 1797 \\
\hline $\begin{array}{l}\text { Kiowa-2006_-2131.FIN2 } \\
\text { Kiowa-2076 } \\
\text { 19.FIN2C }\end{array}$ & $\begin{array}{r}184 \\
25.72\end{array}$ & $\begin{array}{l}0.51 \\
0.67\end{array}$ & $\begin{array}{l}0.1105 \\
0.115\end{array}$ & $\begin{array}{l}0.00065 \\
0.0015\end{array}$ & $\begin{array}{r}4.585 \\
4.93\end{array}$ & $\begin{array}{r}0.0275 \\
0.055\end{array}$ & $\begin{array}{l}0.3026 \\
0.3165\end{array}$ & $\begin{array}{l}0.00195 \\
0.0036\end{array}$ & $\begin{array}{l}0.54312 \\
0.30088\end{array}$ & $\begin{array}{l}1749 \\
1806\end{array}$ & $\begin{array}{r}5 \\
9.5\end{array}$ & $\begin{array}{l}1703 \\
1771\end{array}$ & $\begin{array}{r}9.5 \\
17.5\end{array}$ & $\begin{array}{l}1802 \\
1877\end{array}$ \\
\hline Kiowa-2076_69.FIN2 & 82.4 & 1.13 & 0.1222 & 0.00115 & 5.88 & 0.055 & 0.3494 & 0.00325 & 0.61439 & 1959 & 8.5 & 1935 & 15.5 & 1993 \\
\hline Kiowa-2076_10.FIN2 & 96.7 & 1.21 & 0.1353 & 0.0013 & 6.39 & 0.065 & 0.3417 & 0.0029 & 0.34137 & 2025 & 8.5 & 1896 & 14 & 2162 \\
\hline Kiowa-2076_118.FIN2 & 104.9 & 1.30 & 0.2106 & 0.0009 & 15.43 & 0.075 & 0.5297 & 0.00245 & 0.62688 & 2841.6 & 4.75 & 2739 & 10.5 & 2914 \\
\hline Kiow-2076_2.FIN2 & 15.5 & 1.16 & 0.0984 & 0.00205 & 2.56 & 0.075 & 0.1866 & 0.0046 & 0.74531 & 1280 & 21.5 & 1097 & 25 & 1568 \\
\hline Kiowa-2076-7.FIN2 & 296 & 0.50 & 0.0624 & 0.00205 & 0.1045 & 0.0038 & 0.01217 & 0.00017 & 0.30199 & 99.1 & 3.3 & 77.9 & 1.05 & $\begin{array}{r}550 \\
\end{array}$ \\
\hline Kiowa-2076_8FIN2 & 23 & 1.74 & 0.1716 & 0.0024 & 6.17 & 0.095 & 0.2594 & 0.0033 & 0.32982 & & & 1487 & 16.5 & 2587 \\
\hline
\end{tabular}

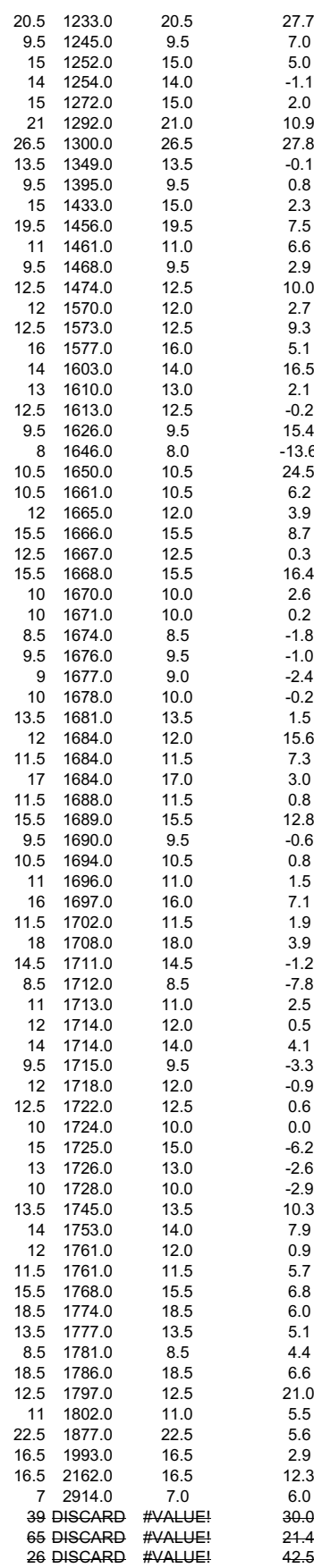




\begin{tabular}{|c|c|c|c|c|c|c|c|c|c|c|c|c|c|c|c|c|c|}
\hline Kiowa-2076_14.FIN2 & 2.97 & 1.02 & 0.36 & 0.01 & 19.7 & 0.7 & 0.392 & 0.008 & 0.26297 & 3008 & 30.5 & 2119 & 37 & 3673 & 41.5 DISCARD & \#VALUE! & 42.3 \\
\hline Kiowa-2076_27.FIN2 & 670 & 0.77 & 0.142 & 0.001 & 1.521 & 0.018 & 0.0774 & 0.0008 & 0.80191 & 936 & 7 & 480.3 & 4.75 & 2248 & 12 DISCARD & \#VALUE! & 48.7 \\
\hline Kiowa-2076_30.FIN2 & 522 & 0.91 & 0.0673 & 0.00105 & 0.284 & 0.0135 & 0.0298 & 0.0011 & 0.9736 & 248 & 10.5 & 189 & 7 & 832 & 32.5 DISCARD & \#VALUE! & 23.8 \\
\hline Kiowa-2076_31.FIN2 & 4.23 & 0.60 & 0.296 & 0.01 & 5.14 & 0.27 & 0.1291 & 0.00395 & 0.90532 & 1752 & 36 & 778 & 22 & 3420 & 50 DISCARD & \#VALUE! & 55.6 \\
\hline Kiowa-2076_37.FIN2 & 328 & 0.26 & 0.1343 & 0.00425 & 3.83 & 0.065 & 0.2195 & 0.0043 & -0.049143 & 1584 & 12.5 & 1277 & 22.5 & 2040 & 48 DISCARD & \#VALUE! & 37.4 \\
\hline Kiowa-2076_40.FIN2 & 401 & 0.41 & 0.0739 & 0.0017 & 0.605 & 0.0125 & 0.0611 & 0.00065 & 0.15873 & 477 & 7.5 & 382 & 3.85 & 988 & 44.5 DISCARD & \#VALUE! & 19.9 \\
\hline Kion-2076_47.FIN2 & 69.3 & 0.60 & 0.124 & 0.007 & 0.197 & 0.013 & 0.01215 & 0.000215 & 0.67334 & 172 & 9 & 77.9 & 1.4 & 1760 & 105 DISCARD & \#VALUE! & 54.7 \\
\hline Kiowa-2076_56.FIN2 & 530 & 0.53 & 0.1116 & 0.0012 & 2.073 & 0.0215 & 0.1366 & 0.00165 & 0.53879 & 1144 & 6.5 & 824 & 9.5 & 1828 & SCARD & \#VALUE! & 3.0 \\
\hline Kiowa2076_63.FIN2 & 311 & 0.75 & 0.0755 & 0.00135 & 0.2703 & 0.0049 & 0.02595 & 0.0003 & 0.35681 & 241.9 & 3.9 & 165.1 & 1.9 & 1072 & 36.5 & & 31.7 \\
\hline Kiowa-2076_72.FIN2 & 4.21 & 10.83 & 0.756 & 0.017 & 9.66 & 0.22 & 0.0969 & 0.00285 & 0.014072 & 2395 & 21 & 593 & 16.5 & 4904 & & \#VALUE! & 75.2 \\
\hline Kiowa-2076_76.FIN2 & 8850 & 10.78 & 1.896 & 0.0345 & 2.906 & 0.015 & 0.01129 & 0.000175 & -0.52763 & 1382.5 & 3.9 & 72.3 & 1.1 & 7440 & ISGARD & \#VALUE! & 94.8 \\
\hline Kiowa-2076_83.FIN2 & 168.1 & 0.85 & 0.1095 & 0.0008 & 3.007 & 0.0295 & 0.1956 & 0.0018 & 0.63972 & 1407 & 7.5 & 1152 & 9.5 & 1794 & 13.5 DISCARD & \#VALUE! & 35.8 \\
\hline Kiowa-2076_84.FIN2 & 5.59 & 0.20 & 0.29 & 0.0055 & 10.01 & 0.215 & 0.2446 & 0.0042 & 0.53392 & 2408 & 17 & 1406 & 21.5 & 3414 & 30 DISCARD & \#VALUE! & 58.8 \\
\hline Kiowa-2076_112.FIN2 & $\begin{array}{l}1150 \\
1015\end{array}$ & 0.04 & 0.0984 & 0.0009 & $\begin{array}{r}2.203 \\
2.31\end{array}$ & 0.0215 & 0.1669 & 0.00165 & 0.66358 & 1183 & $\begin{array}{l}7 \\
8\end{array}$ & $\begin{array}{r}994 \\
1044\end{array}$ & $\begin{array}{l}9 \\
9\end{array}$ & 1589 & 17 DISCARD & \#VALUE! & 37.4 \\
\hline Kiowa-2076_115.FIN2 & 1015 & 0.77 & 0.097 & 0.0008 & 2.31 & 0.0275 & 0.1738 & 0.00165 & 0.69731 & 1218 & 8 & 1034 & & 1570 & 15 DISCARD & \#VALUE! & 4.1 \\
\hline
\end{tabular}

$\dagger 850 \mathrm{Ma}$ cutoff used between $206 \mathrm{~Pb} / 238 \mathrm{U}$ and $207 \mathrm{~Pb} / 206 \mathrm{~Pb}$ ages
Discordance is reported as $206 \mathrm{~Pb} / 238 \mathrm{U}$ vs $207 \mathrm{~Pb} / 235 \mathrm{U}$ for $206 \mathrm{~Pb} / 238 \mathrm{U}$ ages and as $206 \mathrm{~Pb} / 238 \mathrm{U} \mathrm{vs} 207 \mathrm{~Pb} / 206 \mathrm{~Pb}$ for $207 \mathrm{~Pb} / 206 \mathrm{~Pb}$ ages

The 
Table SM2: Detrital Zircon U-Pb Secondary Standard Results

\begin{tabular}{|c|c|c|c|c|c|c|c|c|c|c|c|c|c|}
\hline \multirow[b]{2}{*}{ Sequence } & \multirow[b]{2}{*}{ Samples } & \multicolumn{4}{|c|}{ Pak } & \multicolumn{4}{|c|}{$\begin{array}{c}\text { Plesovice } \\
\end{array}$} & \multicolumn{4}{|c|}{91500} \\
\hline & & $\mathrm{N}$ & Age (Ma) & $\pm 2 \sigma$ internal & MSWD & $\mathrm{N}$ & Age (Ma) & $\pm 2 \sigma$ internal & MSWD & $\mathrm{N}$ & Age (Ma) & $\pm 2 \sigma$ internal & MSWD \\
\hline 06052016_KIOWA & Kiowa-1200, Kiowa-1395 & 10 & 42.1 & 0.23 & 3.3 & 6 & 333.7 & 1.2 & 57.6 & -- & -- & -- & -- \\
\hline 06272016_GS1 & Kiowa-1864 (part 1), Kiowa-2076 & 12 & 42.3 & 0.22 & 1.4 & -- & -- & -- & -- & 15 & 1063.8 & 9.9 & 0.7 \\
\hline 11302016_GS1 & Kiowa-262, Kiowa-114 & -- & -- & -- & -- & 8 & 342.7 & 2.1 & 3.1 & 8 & 1053.0 & 22.9 & 1.8 \\
\hline 11302016_GS2 & Kiowa-848, Kiowa-973, Kiowa-1864 & -- & -- & -- & -- & 10 & 341.8 & 1.6 & 1.0 & 9 & 1041.4 & 18.6 & 2.2 \\
\hline 12012016_GS1 & Kiowa-1635, Kiowa-1777 & -- & -- & -- & -- & 10 & 339.7 & 1.5 & 19.5 & 9 & 1055.9 & 18.2 & 3.4 \\
\hline 12012016_GS2 & Kiowa-1481, Kiowa-1717 & -- & -- & -- & -- & 10 & 332.4 & 2.2 & 8.4 & 9 & 1071.7 & 28.2 & 4.1 \\
\hline 02242017_GS2 & DB-D2-10 & -- & -- & -- & -- & 7 & 341.6 & 1.0 & 10.1 & 10 & 1036.3 & 3.4 & 6.2 \\
\hline $03172017^{-} \mathrm{GS} 1$ & Kiowa-1481 (reanalysis of young grains) & -- & -- & -- & -- & 12 & 324.6 & 1.6 & 3.4 & 12 & 1037.0 & 10.7 & 3.2 \\
\hline
\end{tabular}

Notes:

Pak accepted ${ }^{206} \mathrm{~Pb} /{ }^{238} \mathrm{U}$ age is $\sim 43 \mathrm{Ma}$ (unpublished TIMS data)

Plesovice accepted ${ }^{206} \mathrm{~Pb} /{ }^{238} \mathrm{U}$ age is $337 \mathrm{Ma}$ (Sláma et al., 2008)

91500 accepted ${ }^{206} \mathrm{~Pb} /{ }^{238} \mathrm{U}$ age is $1065 \mathrm{Ma}$ (Wiedenbeck et al., 1995)

References:

Sláma, J., et al., 2008, Plešovice zircon-A new natural reference material for U-Pb and Hf isotopic microanalysis, Chemical Geol., 249, p. 1-35, doi:10.1016/j.chemgeo.2007.11.005.

Wiedenbeck, M., Alle, P., Corfu, F., Griffin, W., Meier, M., Oberli, F., Von Quadt, A., Roddick, J., and Spiegel, W., 1995, Three natural zircon standards for U-Th-Pb, Lu-Hf, trace element and REE

analyses: Geostandards Newsletter, v. 19, p. 1-23, doi:10.1111/j.1751-908X.1995.tb00147.x. 
Table SM3: Detrital Zircon (U-Th[Sm])/He Analytical Results

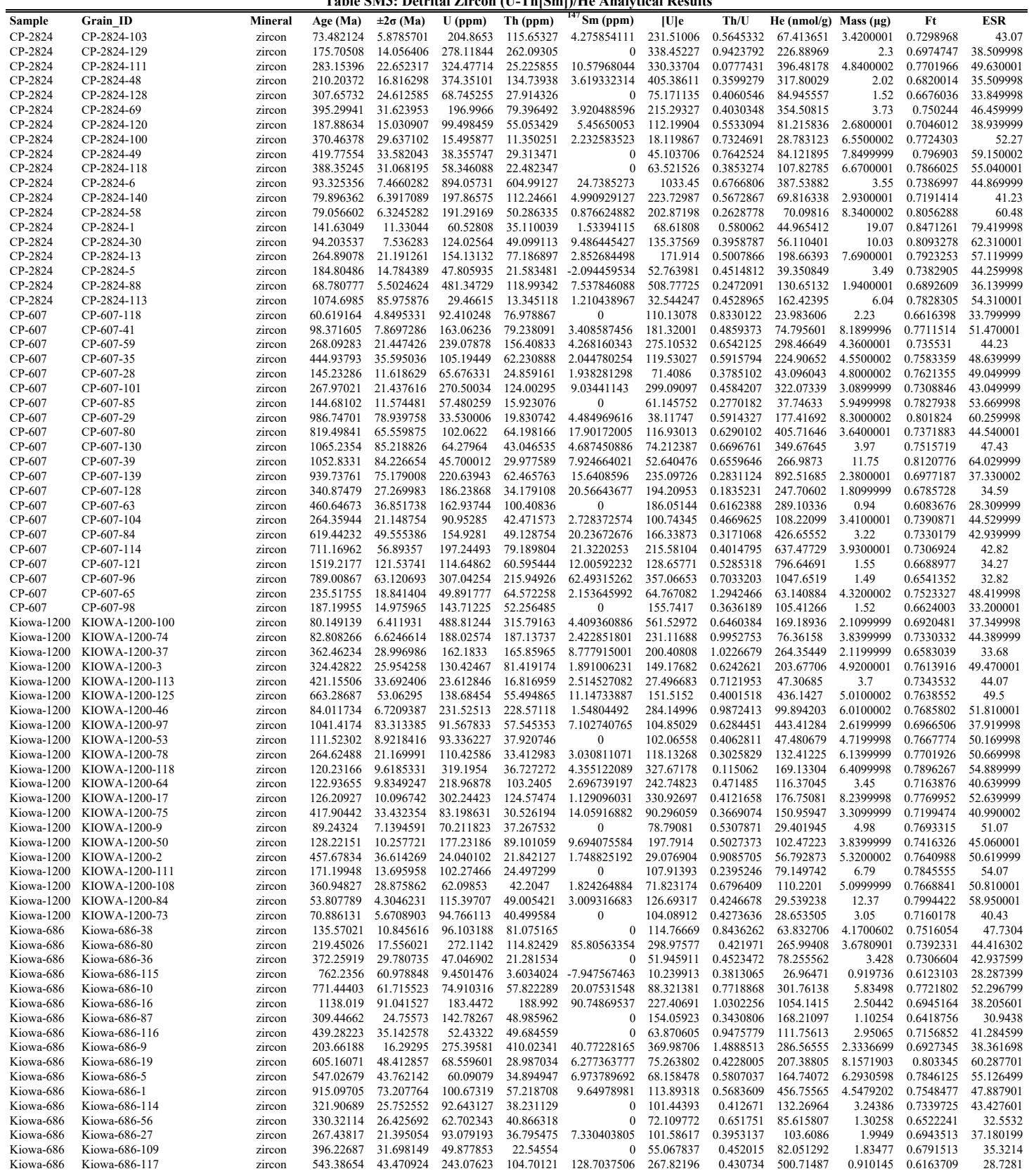

*U-Pb age is reported from the rim age. An older core is also present. 
Table SM4: Non-negative Matrix Factorization Results

\begin{tabular}{|c|c|c|c|c|c|c|c|c|c|c|c|c|c|c|c|c|c|c|c|c|c|}
\hline & Wa- & Kiowa-2 & 3-D2 & owa- & wat & jwa- & Giowa- & wa-12 & tiowa- & tiowa-1 & Kiowa- & $\overline{\overline{\text { Kiowa-11 }}}$ & 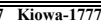 & 7 Kiowa-1864 & K Kiowa-2076 & 6 & $\overline{32 \text { CP }-37}$ & 7 (7CP-60 & $\overline{77 \text { (PP-15 }}$ & 22 (PP-234 & $\overline{9 \mathrm{CPP}-2}$ \\
\hline $\mathbf{N}$ & 124 & 58 & 123 & 89 & 104 & 85 & 97 & 138 & 107 & 133 & 138 & 136 & 137 & 59 & 114 & 133 & 136 & 132 & 141 & 141 & 139 \\
\hline $1 \mathrm{EM} \mathrm{r2}$ & 0.45 & 0.38 & 0.51 & 0.84 & 0.77 & 0.26 & 0.21 & 0.21 & 0.72 & 0.43 & 0.28 & 0.56 & 0.23 & 0.17 & 0.17 & 0.77 & 0.79 & 0.65 & 0.75 & 0.33 & 0.18 \\
\hline 1EM Vmax & 0.36 & 0.27 & 0.23 & 0.21 & 0.15 & 0.30 & 0.36 & 0.37 & 0.36 & 0.26 & 0.31 & 0.21 & 0.36 & 0.42 & 0.36 & 0.47 & 0.45 & 0.19 & 0.19 & 0.27 & 0.36 \\
\hline 1EM EM1 & 1.00 & 1.00 & 1.00 & 1.00 & 1.00 & 1.00 & 1.00 & 1.00 & 1.00 & 1.00 & 1.00 & 1.00 & 1.00 & 1.00 & 1.00 & 1.00 & 1.00 & 1.00 & 1.00 & 1.00 & 1.00 \\
\hline 2EM r2 & 0.48 & 0.66 & 0.51 & 0.91 & 0.78 & 0.51 & 0.54 & 0.83 & 0.90 & 0.71 & 0.90 & 0.56 & 0.66 & 0.85 & 0.76 & 0.96 & 0.95 & 0.65 & 0.78 & 0.48 & 0.89 \\
\hline 2EM Vmax & 0.32 & 0.12 & 0.21 & 0.11 & 0.18 & 0.19 & 0.24 & 0.11 & 0.16 & 0.10 & 0.11 & 0.21 & 0.15 & 0.14 & 0.15 & 0.19 & 0.16 & 0.20 & 0.21 & 0.21 & 0.12 \\
\hline 2EM EM1 & 0.47 & 0.23 & 0.54 & 0.83 & 0.70 & 0.20 & 0.12 & 0.02 & 1.00 & 0.24 & 0.06 & 0.61 & 0.10 & 0.00 & 0.00 & 1.00 & 1.00 & 0.65 & 0.74 & 0.28 & 0.00 \\
\hline 2EM EM2 & 0.53 & 0.77 & 0.46 & 0.17 & 0.30 & 0.80 & 0.88 & 0.98 & 0.00 & 0.76 & 0.94 & 0.39 & 0.90 & 1.00 & 1.00 & 0.00 & 0.00 & 0.35 & 0.26 & 0.72 & 1.00 \\
\hline 3EM r2 & 0.60 & 0.68 & 0.94 & 0.92 & 0.78 & 0.55 & 0.53 & 0.84 & 0.92 & 0.73 & 0.90 & 0.61 & 0.67 & 0.85 & 0.80 & 0.97 & 0.95 & 0.77 & 0.79 & 0.50 & 0.90 \\
\hline 3EM Vmax & 0.26 & 0.10 & 0.08 & 0.10 & 0.18 & 0.17 & 0.24 & 0.12 & 0.12 & 0.11 & 0.11 & 0.19 & 0.14 & 0.14 & 0.17 & 0.16 & 0.18 & 0.14 & 0.19 & 0.20 & 0.12 \\
\hline 3EM EM1 & 0.23 & 0.20 & 0.19 & 0.63 & 0.57 & 0.20 & 0.06 & 0.01 & 1.00 & 0.14 & 0.03 & 0.58 & 0.02 & 0.00 & 0.01 & 0.94 & 0.84 & 0.38 & 0.66 & 0.25 & 0.00 \\
\hline 3EM EM2 & 0.19 & 0.71 & 0.00 & 0.06 & 0.23 & 0.80 & 0.67 & 0.88 & 0.00 & 0.52 & 0.77 & 0.42 & 0.61 & 0.85 & 0.99 & 0.00 & 0.00 & 0.07 & 0.26 & 0.68 & 0.94 \\
\hline 3EM EM3 & 0.58 & 0.10 & 0.81 & 0.31 & 0.20 & 0.00 & 0.26 & 0.11 & 0.00 & 0.34 & 0.20 & 0.00 & 0.37 & 0.15 & 0.00 & 0.06 & 0.16 & 0.55 & 0.09 & 0.08 & 0.06 \\
\hline $4 \mathrm{EM} \mathrm{r} 2$ & 0.61 & 0.68 & 0.94 & 0.92 & 0.81 & 0.78 & 0.79 & 0.85 & 0.93 & 0.74 & 0.91 & 0.70 & 0.70 & 0.89 & 0.86 & 0.97 & 0.95 & 0.78 & 0.86 & 0.78 & 0.93 \\
\hline 4EM Vmax & 0.26 & 0.11 & 0.08 & 0.08 & 0.11 & 0.07 & 0.16 & 0.09 & 0.12 & 0.13 & 0.06 & 0.13 & 0.08 & 0.07 & 0.08 & 0.11 & 0.14 & 0.15 & 0.09 & 0.06 & 0.06 \\
\hline 4EM EMI & 0.24 & 0.15 & 0.20 & 0.62 & 0.44 & 0.05 & 0.00 & 0.01 & 0.97 & 0.14 & 0.04 & 0.36 & 0.00 & 0.00 & 0.03 & 0.94 & 0.83 & 0.33 & 0.46 & 0.08 & 0.00 \\
\hline 4EM EM2 & 0.23 & 0.54 & 0.00 & 0.07 & 0.05 & 0.23 & 0.17 & 0.75 & 0.03 & 0.47 & 0.68 & 0.07 & 0.34 & 0.81 & 0.95 & 0.00 & 0.00 & 0.00 & 0.00 & 0.15 & 0.83 \\
\hline 4EM EM3 & 0.53 & 0.07 & 0.80 & 0.29 & 0.16 & 0.00 & 0.17 & 0.08 & 0.00 & 0.30 & 0.17 & 0.00 & 0.28 & 0.14 & 0.00 & 0.06 & 0.16 & 0.50 & 0.07 & 0.05 & 0.05 \\
\hline 4EM EM4 & 0.00 & 0.23 & 0.00 & 0.02 & 0.35 & 0.72 & 0.66 & 0.16 & 0.00 & 0.09 & 0.11 & 0.56 & 0.37 & 0.05 & 0.02 & 0.00 & 0.01 & 0.17 & 0.47 & 0.72 & 0.12 \\
\hline $5 \mathrm{EM} \mathrm{r2}$ & 0.98 & 0.68 & 0.97 & 0.93 & 0.82 & 0.79 & 0.78 & 0.90 & 0.93 & 0.74 & 0.91 & 0.74 & 0.70 & 0.89 & 0.85 & 0.97 & 0.95 & 0.80 & 0.86 & 0.78 & 0.93 \\
\hline 5EM Vmax & 0.06 & 0.11 & 0.05 & 0.06 & 0.12 & 0.07 & 0.17 & 0.07 & 0.12 & 0.13 & 0.06 & 0.12 & 0.09 & 0.08 & 0.09 & 0.10 & 0.14 & 0.13 & 0.09 & 0.06 & 0.05 \\
\hline 5EM EMI & 0.14 & 0.14 & 0.14 & 0.58 & 0.41 & 0.05 & 0.00 & 0.01 & 0.96 & 0.11 & 0.02 & 0.30 & 0.00 & 0.00 & 0.02 & 0.92 & 0.78 & 0.28 & 0.42 & 0.07 & 0.00 \\
\hline 5EM EM2 & 0.00 & 0.51 & 0.00 & 0.09 & 0.07 & 0.26 & 0.22 & 0.78 & 0.04 & 0.41 & 0.58 & 0.01 & 0.35 & 0.71 & 0.78 & 0.00 & 0.00 & 0.00 & 0.00 & 0.17 & 0.69 \\
\hline 5EM EM3 & 0.13 & 0.05 & 0.78 & 0.33 & 0.18 & 0.01 & 0.18 & 0.15 & 0.00 & 0.27 & 0.12 & 0.00 & 0.28 & 0.10 & 0.00 & 0.08 & 0.15 & 0.51 & 0.06 & 0.08 & 0.00 \\
\hline 5EM EM4 & 0.00 & 0.18 & 0.00 & 0.00 & 0.33 & 0.67 & 0.60 & 0.06 & 0.00 & 0.05 & 0.06 & 0.50 & 0.29 & 0.00 & 0.00 & 0.00 & 0.01 & 0.15 & 0.45 & 0.68 & 0.05 \\
\hline 5EM EM5 & 0.74 & 0.12 & 0.08 & 0.00 & 0.00 & 0.00 & 0.00 & 0.00 & 0.00 & 0.17 & 0.23 & 0.20 & 0.08 & 0.19 & 0.20 & 0.00 & 0.05 & 0.06 & 0.07 & 0.01 & 0.26 \\
\hline 6EM r2 & 0.99 & 0.69 & 0.97 & 0.95 & 0.85 & 0.82 & 0.83 & 0.89 & 0.95 & 0.78 & 0.93 & 0.88 & 0.71 & 0.89 & 0.85 & 0.97 & 0.96 & 0.84 & 0.90 & 0.78 & 0.93 \\
\hline 6EM Vmax & 0.02 & 0.11 & 0.04 & 0.06 & 0.08 & 0.07 & 0.12 & 0.07 & 0.12 & 0.09 & 0.06 & 0.08 & 0.08 & 0.07 & 0.08 & 0.14 & 0.18 & 0.11 & 0.07 & 0.05 & 0.05 \\
\hline 6EM EM1 & 0.02 & 0.00 & 0.00 & 0.00 & 0.00 & 0.06 & 0.02 & 0.01 & 0.00 & 0.27 & 0.18 & 0.58 & 0.00 & 0.00 & 0.04 & 0.22 & 0.30 & 0.32 & 0.42 & 0.33 & 0.04 \\
\hline 6EM EM2 & 0.00 & 0.40 & 0.00 & 0.04 & 0.00 & 0.13 & 0.08 & 0.71 & 0.01 & 0.34 & 0.53 & 0.03 & 0.27 & 0.71 & 0.76 & 0.00 & 0.00 & 0.01 & 0.00 & 0.08 & 0.65 \\
\hline 6EM EM3 & 0.05 & 0.02 & 0.69 & 0.23 & 0.12 & 0.00 & 0.15 & 0.16 & 0.00 & 0.24 & 0.11 & 0.00 & 0.26 & 0.10 & 0.00 & 0.04 & 0.09 & 0.41 & 0.06 & 0.06 & 0.00 \\
\hline 6EM EM4 & 0.00 & 0.23 & 0.01 & 0.08 & 0.34 & 0.70 & 0.69 & 0.11 & 0.05 & 0.00 & 0.01 & 0.15 & 0.36 & 0.01 & 0.00 & 0.00 & 0.00 & 0.02 & 0.18 & 0.49 & 0.07 \\
\hline 6EM EM5 & 0.69 & 0.14 & 0.10 & 0.00 & 0.01 & 0.02 & 0.05 & 0.00 & 0.00 & 0.09 & 0.17 & 0.06 & 0.11 & 0.18 & 0.17 & 0.00 & 0.00 & 0.00 & 0.00 & 0.00 & 0.23 \\
\hline 6EM EM6 & 0.24 & 0.21 & 0.20 & 0.65 & 0.53 & 0.10 & 0.00 & 0.01 & 0.94 & 0.06 & 0.00 & 0.19 & 0.00 & 0.00 & 0.03 & 0.74 & 0.61 & 0.24 & 0.34 & 0.04 & 0.00 \\
\hline $7 \mathrm{EM} \mathrm{r2}$ & 0.99 & 0.69 & 0.96 & 0.96 & 0.87 & 0.84 & 0.83 & 0.89 & 1.00 & 0.83 & 0.93 & 0.92 & 0.74 & 0.89 & 0.86 & 0.98 & 0.97 & 0.82 & 0.90 & 0.79 & 0.93 \\
\hline 7EM Vmax & 0.04 & 0.11 & 0.06 & 0.04 & 0.09 & 0.07 & 0.13 & 0.07 & 0.04 & 0.09 & 0.05 & 0.06 & 0.07 & 0.07 & 0.08 & 0.12 & 0.15 & 0.12 & 0.07 & 0.05 & 0.05 \\
\hline 7EM EM1 & 0.13 & 0.07 & 0.25 & 0.64 & 0.47 & 0.08 & 0.00 & 0.03 & 0.24 & 0.00 & 0.02 & 0.00 & 0.00 & 0.00 & 0.03 & 0.74 & 0.63 & 0.27 & 0.28 & 0.06 & 0.00 \\
\hline 7 TEM EM2 & 0.00 & 0.38 & 0.00 & 0.06 & 0.00 & 0.12 & 0.07 & 0.69 & 0.00 & 0.31 & 0.53 & 0.03 & 0.23 & 0.66 & 0.77 & 0.00 & 0.00 & 0.00 & 0.00 & 0.08 & 0.64 \\
\hline 7EM EM3 & 0.03 & 0.04 & 0.65 & 0.19 & 0.07 & 0.00 & 0.18 & 0.16 & 0.00 & 0.27 & 0.13 & 0.00 & 0.27 & 0.14 & 0.00 & 0.00 & 0.05 & 0.42 & 0.03 & 0.04 & 0.01 \\
\hline 7EM EM4 & 0.00 & 0.20 & 0.00 & 0.10 & 0.41 & 0.69 & 0.60 & 0.11 & 0.01 & 0.00 & 0.01 & 0.14 & 0.32 & 0.01 & 0.00 & 0.00 & 0.00 & 0.04 & 0.22 & 0.52 & 0.08 \\
\hline 7EM EM5 & 0.69 & 0.11 & 0.09 & 0.01 & 0.03 & 0.01 & 0.00 & 0.00 & 0.00 & 0.05 & 0.16 & 0.03 & 0.08 & 0.15 & 0.18 & 0.00 & 0.00 & 0.00 & 0.00 & 0.00 & 0.23 \\
\hline 7EM EM6 & 0.11 & 0.18 & 0.00 & 0.00 & 0.02 & 0.00 & 0.07 & 0.00 & 0.75 & 0.19 & 0.00 & 0.30 & 0.10 & 0.03 & 0.00 & 0.03 & 0.01 & 0.01 & 0.06 & 0.00 & 0.00 \\
\hline 7ЕM EM7 & 0.05 & 0.01 & 0.00 & 0.00 & 0.00 & 0.10 & 0.07 & 0.00 & 0.00 & 0.18 & 0.16 & 0.51 & 0.00 & 0.00 & 0.02 & 0.23 & 0.31 & 0.26 & 0.41 & 0.30 & 0.05 \\
\hline $8 E M$ r2 & 0.99 & 0.95 & 0.97 & 0.97 & 0.87 & 0.85 & 0.84 & 0.91 & 1.00 & 0.83 & 0.93 & 0.92 & 0.76 & 0.90 & 0.86 & 0.98 & 0.97 & 0.83 & 0.91 & 0.80 & 0.93 \\
\hline 8EM Vmax & 0.04 & 0.04 & 0.05 & 0.04 & 0.08 & 0.06 & 0.12 & 0.07 & 0.04 & 0.09 & 0.05 & 0.07 & 0.06 & 0.06 & 0.08 & 0.12 & 0.15 & 0.12 & 0.06 & 0.05 & 0.05 \\
\hline 8EM EM1 & 0.11 & 0.10 & 0.24 & 0.62 & 0.41 & 0.07 & 0.00 & 0.03 & 0.21 & 0.00 & 0.01 & 0.00 & 0.00 & 0.00 & 0.02 & 0.71 & 0.60 & 0.22 & 0.23 & 0.05 & 0.00 \\
\hline 8EM EM2 & 0.00 & 0.05 & 0.00 & 0.07 & 0.03 & 0.15 & 0.00 & 0.68 & 0.00 & 0.27 & 0.44 & 0.00 & 0.26 & 0.63 & 0.63 & 0.00 & 0.00 & 0.01 & 0.00 & 0.10 & 0.56 \\
\hline 8EM EM3 & 0.06 & 0.00 & 0.64 & 0.19 & 0.06 & 0.00 & 0.12 & 0.14 & 0.00 & 0.24 & 0.11 & 0.00 & 0.23 & 0.12 & 0.00 & 0.00 & 0.06 & 0.39 & 0.03 & 0.02 & 0.00 \\
\hline 8EM EM4 & 0.00 & 0.00 & 0.00 & 0.11 & 0.39 & 0.63 & 0.44 & 0.15 & 0.03 & 0.00 & 0.01 & 0.09 & 0.33 & 0.07 & 0.00 & 0.00 & 0.00 & 0.06 & 0.23 & 0.48 & 0.09 \\
\hline 8EM EM5 & 0.64 & 0.00 & 0.07 & 0.01 & 0.05 & 0.04 & 0.00 & 0.00 & 0.00 & 0.03 & 0.14 & 0.01 & 0.09 & 0.15 & 0.15 & 0.00 & 0.00 & 0.01 & 0.02 & 0.02 & 0.21 \\
\hline 8EM EM6 & 0.14 & 0.00 & 0.00 & 0.00 & 0.07 & 0.00 & 0.02 & 0.00 & 0.76 & 0.17 & 0.00 & 0.26 & 0.09 & 0.03 & 0.00 & 0.05 & 0.03 & 0.05 & 0.11 & 0.00 & 0.00 \\
\hline 8EM EM7 & 0.05 & 0.00 & 0.00 & 0.00 & 0.00 & 0.06 & 0.03 & 0.00 & 0.00 & 0.17 & 0.13 & 0.49 & 0.00 & 0.00 & 0.00 & 0.24 & 0.32 & 0.25 & 0.39 & 0.27 & 0.02 \\
\hline 8EM EM8 & 0.00 & 0.85 & 0.05 & 0.00 & 0.00 & 0.06 & 0.39 & 0.00 & 0.00 & 0.12 & 0.17 & 0.15 & 0.00 & 0.00 & 0.20 & 0.00 & 0.00 & 0.00 & 0.00 & 0.06 & 0.12 \\
\hline $9 \mathrm{EM} \mathrm{r2}$ & 0.99 & 0.89 & 0.98 & 0.97 & 0.87 & 0.85 & 0.97 & 0.94 & 1.00 & 0.84 & 0.92 & 0.94 & 0.79 & 0.91 & 0.90 & 0.98 & 0.98 & 0.85 & 0.93 & 0.85 & 0.93 \\
\hline 9EM Vmax & 0.03 & 0.07 & 0.05 & 0.03 & 0.08 & 0.04 & 0.03 & 0.05 & 0.04 & 0.09 & 0.05 & 0.05 & 0.07 & 0.07 & 0.07 & 0.09 & 0.12 & 0.14 & 0.06 & 0.05 & 0.05 \\
\hline 9EM EM1 & 0.13 & 0.00 & 0.21 & 0.59 & 0.37 & 0.00 & 0.00 & 0.05 & 0.21 & 0.00 & 0.01 & 0.00 & 0.00 & 0.00 & 0.00 & 0.74 & 0.59 & 0.18 & 0.22 & 0.00 & 0.00 \\
\hline 9EM EM2 & 0.00 & 0.00 & 0.00 & 0.04 & 0.00 & 0.00 & 0.00 & 0.66 & 0.00 & 0.31 & 0.40 & 0.00 & 0.27 & 0.63 & 0.44 & 0.00 & 0.00 & 0.01 & 0.00 & 0.00 & 0.49 \\
\hline 9EM EM3 & 0.00 & 0.11 & 0.64 & 0.19 & 0.00 & 0.00 & 0.00 & 0.00 & 0.00 & 0.23 & 0.09 & 0.00 & 0.08 & 0.04 & 0.00 & 0.00 & 0.06 & 0.37 & 0.01 & 0.00 & 0.00 \\
\hline 9EM EM4 & 0.00 & 0.13 & 0.08 & 0.05 & 0.19 & 0.27 & 0.62 & 0.29 & 0.00 & 0.03 & 0.08 & 0.06 & 0.29 & 0.05 & 0.00 & 0.00 & 0.00 & 0.00 & 0.00 & 0.16 & 0.09 \\
\hline 9EM EM5 & 0.71 & 0.00 & 0.05 & 0.00 & 0.03 & 0.00 & 0.07 & 0.00 & 0.00 & 0.03 & 0.12 & 0.01 & 0.14 & 0.15 & 0.06 & 0.00 & 0.00 & 0.00 & 0.00 & 0.00 & 0.16 \\
\hline 9EM EM6 & 0.10 & 0.16 & 0.00 & 0.00 & 0.07 & 0.03 & 0.02 & 0.00 & 0.77 & 0.14 & 0.00 & 0.22 & 0.06 & 0.02 & 0.00 & 0.00 & 0.00 & 0.08 & 0.11 & 0.00 & 0.00 \\
\hline 9EM EM7 & 0.06 & 0.02 & 0.00 & 0.00 & 0.00 & 0.02 & 0.15 & 0.00 & 0.00 & 0.20 & 0.12 & 0.53 & 0.00 & 0.00 & 0.00 & 0.26 & 0.34 & 0.17 & 0.35 & 0.20 & 0.01 \\
\hline 9EM EM8 & 0.00 & 0.57 & 0.01 & 0.06 & 0.10 & 0.34 & 0.14 & 0.00 & 0.00 & 0.05 & 0.18 & 0.13 & 0.00 & 0.05 & 0.49 & 0.00 & 0.00 & 0.00 & 0.04 & 0.28 & 0.22 \\
\hline 9EM EM9 & 0.00 & 0.00 & 0.00 & 0.07 & 0.23 & 0.34 & 0.00 & 0.00 & 0.01 & 0.00 & 0.00 & 0.06 & 0.16 & 0.05 & 0.01 & 0.00 & 0.00 & 0.19 & 0.27 & 0.36 & 0.03 \\
\hline 10EM r2 & 0.99 & 0.99 & 0.98 & 0.97 & 0.88 & 0.86 & 0.97 & 0.95 & 1.00 & 0.84 & 0.93 & 0.93 & 0.88 & 0.89 & 0.93 & 0.98 & 0.98 & 0.86 & 0.93 & 0.86 & 0.93 \\
\hline 10EM Vmax & 0.03 & 0.01 & 0.05 & 0.03 & 0.08 & 0.04 & 0.04 & 0.06 & 0.04 & 0.09 & 0.05 & 0.05 & 0.06 & 0.08 & 0.05 & 0.10 & 0.12 & 0.14 & 0.06 & 0.04 & 0.05 \\
\hline 10EM EM1 & 0.13 & 0.00 & 0.22 & 0.59 & 0.38 & 0.03 & 0.00 & 0.04 & 0.21 & 0.00 & 0.01 & 0.00 & 0.00 & 0.00 & 0.02 & 0.72 & 0.58 & 0.17 & 0.21 & 0.00 & 0.00 \\
\hline 10EM EM2 & 0.00 & 0.00 & 0.00 & 0.06 & 0.00 & 0.00 & 0.13 & 0.65 & 0.00 & 0.14 & 0.23 & 0.00 & 0.58 & 0.34 & 0.00 & 0.00 & 0.00 & 0.00 & 0.00 & 0.00 & 0.25 \\
\hline 10EM EM3 & 0.00 & 0.00 & 0.64 & 0.17 & 0.03 & 0.00 & 0.09 & 0.00 & 0.00 & 0.24 & 0.08 & 0.00 & 0.00 & 0.06 & 0.05 & 0.00 & 0.06 & 0.36 & 0.00 & 0.00 & 0.00 \\
\hline 10EM EM4 & 0.00 & 0.00 & 0.06 & 0.03 & 0.26 & 0.47 & 0.58 & 0.00 & 0.02 & 0.00 & 0.00 & 0.08 & 0.00 & 0.00 & 0.12 & 0.00 & 0.00 & 0.00 & 0.05 & 0.21 & 0.05 \\
\hline 10EM EM5 & 0.72 & 0.00 & 0.05 & 0.00 & 0.04 & 0.00 & 0.07 & 0.00 & 0.00 & 0.00 & 0.08 & 0.02 & 0.20 & 0.09 & 0.00 & 0.00 & 0.00 & 0.00 & 0.00 & 0.00 & 0.12 \\
\hline 10EM EM6 & 0.09 & 0.14 & 0.00 & 0.00 & 0.06 & 0.00 & 0.00 & 0.00 & 0.77 & 0.14 & 0.00 & 0.21 & 0.06 & 0.03 & 0.00 & 0.00 & 0.00 & 0.11 & 0.11 & 0.00 & 0.00 \\
\hline
\end{tabular}




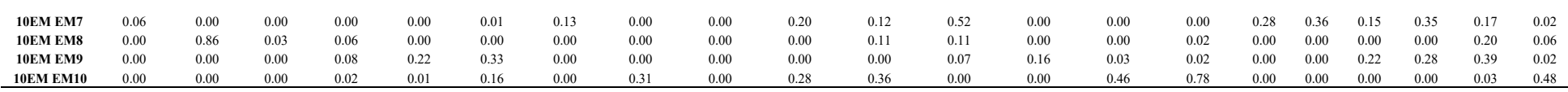

$\begin{array}{llllll}\text { 10EM EM10 } & 0.00 & 0.00 & 0.00 & 0.02 & 0.01\end{array}$

Notes
$1 E M=1$ end-member scenario, $2 E \mathrm{M}=2$ end-member scenario, etc.

r2 = Cross-correlation between the modeled and measured sample

$\mathrm{EM} 1=$ end-member $1, \mathrm{EM} 2$ = end-member 2, etc. 
Table SM5: Sample Groups for End-member Comparison

\begin{tabular}{|c|c|c|c|}
\hline End-member & Unit & Sample(s) & Source \\
\hline \multicolumn{4}{|c|}{ End-member 1 (EMI) } \\
\hline & South Platte River & DB-SPR-01 & Sharman et al. (2018) \\
\hline \multicolumn{4}{|c|}{ End-member 2 (EM2) } \\
\hline \multicolumn{4}{|c|}{ Paleocene Rockdale delta } \\
\hline & Wilcox Grp. (Calvert Bluff Fm.) & CB-BBM & Wahl et al. (2016) \\
\hline & Wilcox Grp. (Calvert Bluff Fm.) & CB-BS & Wahl et al. (2016) \\
\hline & Wilcox Grp. (Simsboro Fm.) & XOM-GOM-67 & Blum and Pecha (2014) \\
\hline & Wilcox Grp. (Simsboro Fm.) & XOM-GOM-69 & Blum and Pecha (2014) \\
\hline & Wilcox Grp. (Simsboro Fm.) & XOM-GOM-70 & Blum and Pecha (2014) \\
\hline & Wilcox Grp. (Simsboro Fm.) & XOM-GOM-71 & Blum and Pecha (2014) \\
\hline & Wilcox Grp. (lower part) & CG11-10 & Craddock and Kylander-Clark (2013) \\
\hline & Wilcox Grp. (Simsboro Fm.) & $\mathrm{Si}-\mathrm{KM}$ & Wahl et al. (2016) \\
\hline & Wilcox Grp. (Simsboro Fm.) & Si-TQ & Wahl et al. (2016) \\
\hline & Wilcox Grp. (Simsboro Fm.) & Si-LP & Wahl et al. (2016) \\
\hline & Wilcox Grp. (Simsboro Fm.) & Si-B & Wahl et al. (2016) \\
\hline & Wilcox Grp. (Hooper Fm.) & H-DR & Wahl et al. (2016) \\
\hline & Wilcox Grp. (Hooper Fm.) & $\mathrm{H}-\mathrm{PC}$ & Wahl et al. (2016) \\
\hline & Wilcox Grp. (Hooper Fm.) & $\mathrm{Se}$ & Wahl et al. (2016) \\
\hline \multicolumn{4}{|c|}{ Upper Cretaceous-Paleocene of southern Rocky Mountain region (San Juan, Galisteo, and Raton basins) } \\
\hline & Nacimiento Formation & WP26 & Donahue (2016) \\
\hline & Nacimento Formation & WP34 & Donahue (2016) \\
\hline & Nacimiento Formation & SJ006 & Sharman et al. (2017) \\
\hline & Animas Formation & WP57 & Donahue (2016) \\
\hline & Animas Formation & WP44 & Donahue (2016) \\
\hline & Animas Formation & WP45 & Donahue (2016) \\
\hline & Ojo Alamo Sandstone & WP27 & Donahue (2016) \\
\hline & Ojo Alamo Sandstone & WP36 & Donahue (2016) \\
\hline & Ojo Alamo Sandstone & WP63a & Donahue (2016) \\
\hline & Ojo Alamo Formation & SJ003 & Sharman et al. (2017) \\
\hline & Animas Formation (McDermott Member) & WP53 & Donahue (2016) \\
\hline & Animas Formation (McDermott Member) & WP56 & Donahue (2016) \\
\hline & McDermott Formation & MS-10-02 & Donahue (2016) \\
\hline & McDermott Formation & MS-10-04 & Donahue (2016) \\
\hline & Poison Canyon Formation & RB201 & Bush et al. (2016) \\
\hline & Poison Canyon Formation & RB108 & Bush et al. (2016) \\
\hline & Poison Canyon Formation & PCSSS-73z & Rasmussen and Foreman (2017) \\
\hline & Poison Canyon Formation (lower part) & RB113 & Bush et al. (2016) \\
\hline & Raton Formation & RB206 & Bush et al. (2016) \\
\hline & Raton Formation & RB207 & Bush et al. (2016) \\
\hline & Raton Formation & RB109 & Bush et al. (2016) \\
\hline & Raton Formation (lower part) & RB112 & Bush et al. (2016) \\
\hline & Raton Formation (lower part) & RB208 & Bush et al. (2016) \\
\hline & Trinidad Sandstone & RB111 & Bush et al. (2016) \\
\hline \multicolumn{4}{|c|}{ End-member 3 (EM3) } \\
\hline & South Platte River & DB-SPR-01 & Sharman et al. (2018) \\
\hline \multicolumn{4}{|c|}{ End-member 4 (EM4) } \\
\hline & Dakota Group & DB-DF-03 & Sharman et al. (2018) \\
\hline & Morrison Formation & DB-MF-01 & Sharman et al. (2018) \\
\hline & Lyons Sandstone & DB-LS-02 & Sharman et al. (2018) \\
\hline
\end{tabular}


References

Blum, M.D., and Pecha, M., 2014, Mid-Cretaceous to Paleocene North American drainage reorganization from detrital zircons: Geology, v. 42, p. 607-610, doi:10.1130/G35513.1.

Bush, M.A., Horton, B.K., Murphy, M.A., and Stockli, D.F., 2016, Detrital record of initial basement exhumation along the Laramide deformation front, southern Rocky Mountains: Tectonics, v. 35, p. 2117-2130, doi:10.1002/2016TC004194.

Craddock, W., and Kylander-Clark, A., 2013, U-Pb ages of detrital zircons from the Tertiary Mississippi River Delta in central Louisiana: Insights into sediment provenance: Geosphere, v. 9, p. 1832-1851.

Donahue, M.M.S., 2016, Episodic uplift of the Rocky Mountains: Evidence from U-Pb detrital zircon geochronology and lowtemperature thermochronology with a chapter on using mobile technology for geoscience and education: The University of New Mexico, Albuquerque. Earth \& Planetary Sciences Ph.D dissertation., p. 188 p., doi:10.1017/CBO9781107415324.004.

Rasmussen, D.M., and Foreman, B.Z., 2017, Provenance of lower Paleogene strata in the Huerfano Basin: Implications for uplift of the Wet Mountains, Colorado, U.S.A.: Journal of Sedimentary Research, v. 87, p. 579-593, doi:10.2110/jsr.2017.30.

Sharman, G.R., Covault, J.A., Stockli, D.F., Wroblewski, F.-A.J., and Bush, M.A., 2017, Early Cenozoic drainage reorganization of the United States Western Interior-Gulf of Mexico sediment routing system: Geology, v. 45, doi:10.1130/G38765.1.

Sharman, G.R., Stockli, D.F., Flaig, P.P., Raynolds, R.G., and Covault, J.A., 2018, Local-to-distant provenance cyclicity of the southern Front Range, central Colorado: Insights from detrital zircon geochronology: Geological Society of America Special Paper, v. 540, p. 1-23.

Wahl, P.J., Yancey, T.E., Pope, M.C., Miller, B. V., and Ayers, W.B., 2016, U-Pb detrital zircon geochronology of the upper Paleocene to lower Eocene Wilcox Group, east-central Texas: Geosphere, v. 12, p. GES01313.1, doi:10.1130/GES01313.1. 
Table SM6: Sample Lithology

\begin{tabular}{ll}
\hline \hline Sample & Description \\
\hline Kiowa-114 & mL-cU, brown sandstone with muscovite flakes \\
Kiowa-262 & fU-cL, tan sandstone with muscovite flakes \\
Kiowa-848 & well-sorted, fL-vfU, brown sandstone with carbonaceous debris \\
Kiowa-973 & well-sorted, fL, gray sandstone \\
Kiowa-1481 & well-sorted, fL-fU, brown sandstone \\
Kiowa-1635 & poorly-sorted, mL-vcU, brown sandstone with pebbles \\
Kiowa-1717 & well-sorted, fL-vfU, brown sandstone with carbonaceous debris \\
Kiowa-1777 & well-sorted, fL-vfU, brown sandstone \\
DB-D2-10 & poorly-sorted, mL-vcU, light tan sandstone \\
\hline
\end{tabular}

Notes: Sample descriptions not available for detrital separates provided by S. Kelley (Table 1) 